\title{
Ein neuer Ansatz zur Zustandsraumdarstellung, Systemanalyse und Regelung von Drei-Wege-Autoabgaskatalysatoren
}

\author{
Vom Fachbereich \\ Elektrotechnik und Informationstechnik \\ der Technischen Universität Darmstadt \\ zur Erlangung des Grades eines Doktor-Ingenieurs (Dr.-Ing.) \\ genehmigte Dissertation \\ von \\ Dipl.-Ing. Radoy Stanchev \\ geboren am 10. Februar 1985 in Sevlievo
}

Referent: $\quad$ Prof. Dr.-Ing. Ulrich Konigorski

Korreferent: $\quad$ Prof. Dr.-Ing. Herbert Vogel

Tag der Einreichung: 1. November 2016

Tag der Prüfung: $\quad$ 6. Dezember 2016

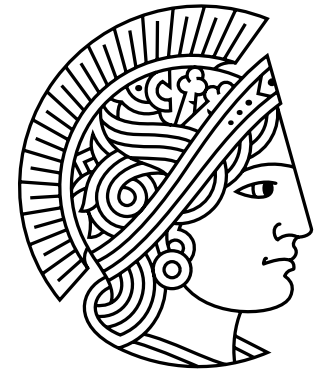

D 17 - Darmstadt 2017 



\section{Vorwort}

Die vorliegende Arbeit entstand während meiner Tätigkeit als wissenschaftlicher Mitarbeiter am Institut für Automatisierungstechnik und Mechatronik, Fachgebiet Regelungstechnik und Mechatronik der Technischen Universität Darmstadt. Die dargestellten Ergebnisse wurden von mir bei der Bearbeitung des vom Bundesministerium für Bildung und Forschung geförderten Projekts „ReffKat“ erzielt.

Es würde mich freuen, wenn die Ergebnisse dieser Arbeit in die Praxis umgesetzt würden und die erarbeiteten Methoden auf andere Problemstellungen angewendet würden. Die Erde mit ihrer biologischen Vielfalt ist im Universum einzigartig. Wir als Menschen stehen in der Verantwortung, sparsam und nachhaltig mit ihren Ressourcen umzugehen.

Ich bedanke mich bei allen, die mich während meiner Zeit als wissenschaftlicher Mitarbeiter und während der Erstellung dieser Arbeit unterstützt haben. Mein besonderer Dank gilt Herrn Prof. Dr.-Ing. Ulrich Konigorski, der stets ein offenes Ohr hatte und für interessante fachliche Diskussionen bereit war.

Bei Herrn Prof. Dr.-Ing. Herbert Vogel vom Ernst-Berl-Institut für Makromolekulare und Technische Chemie an der Technischen Universität Darmstadt bedanke ich mich für die freundliche Übernahme des Korreferates und das Interesse an meiner Arbeit.

Das Projekt „ReffKat“ wurde unter der Federführung der Firma Umicore AG \& Co. KG durchgeführt und ohne die aktive Beteiligung zahlreicher Mitarbeiter wäre das Projekt sicher nicht gelungen. An dieser Stelle bedanke ich mich bei allen beteiligten Mitarbeitern für die gute Zusammenarbeit. Insbesondere möchte ich Herr Dr. Martin Votsmeier hervorheben, der das Projekt ins Leben gerufen hat und als Projektleiter für sein Gelingen sorgte. Weiterhin wären die Messungen am Laborprüfstand ohne die Unterstützung von Jürgen Schell, Roland Adam und Barry van Setten nicht gelungen, wofür ich mich bei ihnen bedanke.

Jan Rink, der als wissenschaftlicher Mitarbeiter am Projekt beteiligt war, möchte ich besonders dafür danken, dass er mir in allen Fragen der Chemie zur Seite stand.

Ich bedanke mich noch bei meinen Institutskollegen, dem Sekretariat und der Werkstatt für die angenehme Arbeitsatmosphäre. Herausheben möchte ich hier Alfred Gross, bei dem ich noch während meines Studiums als wissenschaftliche Hilfskraft erste Einblicke in die Arbeitsweise am Institut erlangen konnte.

Abschließend bedanke ich mich bei meiner Familie, die mir das Studium ermöglicht hat und stets Zuversicht in das Gelingen meiner Promotion hatte.

Darmstadt, im November 2016

Radoy Stanchev 


\section{Inhaltsverzeichnis}

$\begin{array}{lll}\text { Symbole und Abkürzungen } & \text { VII }\end{array}$

Zusammenfassung $\quad$ X

1 Einführung $\quad 1$

1.1 Einleitung . . . . . . . . . . . . . . . . . . . . 1

1.1.1 Schadstoffe im Motorabgas . . . . . . . . . . . . . . . . 1

1.1.2 Anfänge der Abgasnachbehandlung . . . . . . . . . . . . . 3

1.1.3 Hauptkomponenten des Systems zur Abgasnachbehandlung . . . . . . . 4

1.1.4 Stand der Technik . . . . . . . . . . . . . . . . . 7

1.2 Problemstellung und Ziele der Arbeit . . . . . . . . . . . . . . . . . . . 9

1.3 Struktur der Arbeit . . . . . . . . . . . . . . . . . . . . . 11

2 Der Lambdaregelkreis $\quad 14$

2.1 Modellierung der Motorkomponenten im Lambdaregelkreis . . . . . . . . . . . . 15

2.2 Regelung des Lambdawertes . . . . . . . . . . . . . . . . . . . . . . . 19

2.2.1 Anforderungen an den Lambdaregelkreis und Randbedingungen . . . . . 20

2.2 .2 Die IMC-Struktur . . . . . . . . . . . . . . . . . . . 23

2.2.3 Entwurf des Lambdareglers . . . . . . . . . . . . . . . . . . . . . 25

2.3 Implementierung des Lambdareglers . . . . . . . . . . . . . . . . . . . . . 31

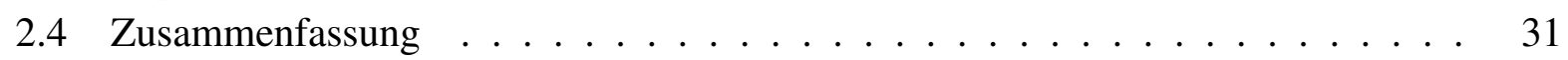

3 Katalysatormodell $\quad 34$

3.1 Modellbildung . . . . . . . . . . . . . . . . . . . 38

3.1.1 Modellierung der Massentransportprozesse . . . . . . . . . . . . 38

3.1.2 Modellierung der chemischen Reaktionen . . . . . . . . . . . . . . . 40

3.1.3 Vereinfachung der Modellgleichungen . . . . . . . . . . . . . . . . 42

3.1 .4 Emissionsmodell für den Motor . . . . . . . . . . . . . . . . . . 43

3.2 Modelldarstellung mittels Gewichtsfunktionen . . . . . . . . . . . . . . . . 45

3.3 Erweiterung auf andere Reaktionsschemata . . . . . . . . . . . . . . . 48

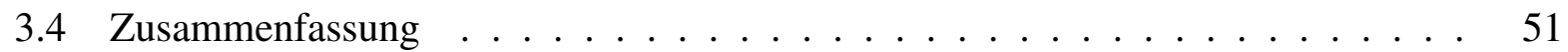

4 Systemanalyse $\quad \mathbf{5 2}$

4.1 Die Gewichtsfunktionen aus Sicht der Systemtheorie und der Chemie . . . . . . 53

4.2 Untersuchung der Stabilität . . . . . . . . . . . . . . . . . . 56

4.3 Linearisierung des Modells . . . . . . . . . . . . . . . . . . . . . . . . . . . . . . . . 59

4.4 Berechnung von Strukturmaßen _ . . . . . . . . . . . . . . . . 62

4.5 Einfluss der Motorarbeitspunktparameter auf die Systemeigenschaften . . . . . . 67 


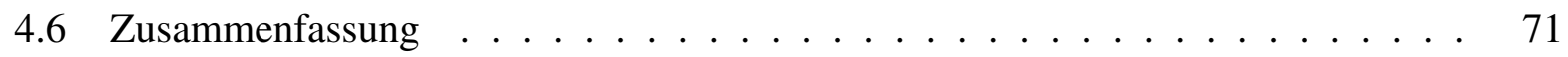

5 Parameteridentifikation $\quad 72$

5.1 Sensitivitätsanalyse . . . . . . . . . . . . . . . . 73

5.2 Identifikationstrajektorie und Identifikationsverfahren . . . . . . . . . . . . . . . . . . . . .

5.3 Parameteridentifikation mit Messdaten . . . . . . . . . . . . . . . 78

5.4 Zusammenfassung . . . . . . . . . . . . . . . . . . 84

6 Regelung des Katalysators $\quad 86$

6.1 Struktur des Regelungskonzeptes . . . . . . . . . . . . . . . . . . . . . . . . . 89

6.2 Regelung des Sauerstoffüllstandes . . . . . . . . . . . . . . . . . . . 90

6.2.1 Reglerentwurf mittels exakter Ein-/Ausgangslinearisierung . . . . . . . . 91

6.3 Regelung der Konvertierungsraten . . . . . . . . . . . . . . . . . . . . 98

6.3.1 LQ-Reglerentwurf . . . . . . . . . . . . . . . . . . . . . 101

6.3.2 Reglerentwurf mittels exakter Ein-/Ausgangslinearisierung . . . . . . . . 107

6.3.3 Nichtlineare modellprädiktive Regelung . . . . . . . . . . . . . . . . . 109

6.4 Vergleich der Regelungskonzepte . . . . . . . . . . . . . . . . . . . 112

6.4.1 Vergleich mit Simulationsdaten . . . . . . . . . . . . . . 112

6.4 Vergleich mit Messdaten . . . . . . . . . . . . . . . . . . . 115

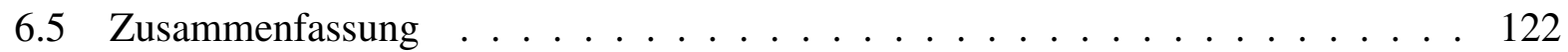

7 Zusammenfassung und Ausblick $\quad 124$

$\begin{array}{lr}\text { Anhang } & 128\end{array}$

A.1 Parameter der Motorarbeitspunkte . . . . . . . . . . . . . . . 128

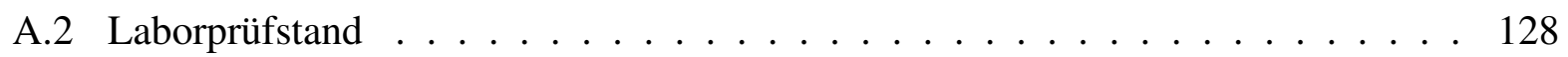

A.3 Regularität der Matrix A . . . . . . . . . . . . . . . . 131

A.4 Beispiel für ein Reaktionsschema mit einer Oberflächenspezies . . . . . . . . . . 132

A.5 Beispiel für ein Reaktionsschema mit mehreren Oberflächenspezies _ . . . . . . 133

$\begin{array}{ll}\text { Literaturverzeichnis } & 135\end{array}$ 


\section{Symbole und Abkürzungen}

Die nachfolgend aufgeführten Größen und Bezeichnungen sind global, d. h. für die ganze Arbeit, gültig. Größen, die nur lokal verwendet werden, sind in der weiteren Arbeit definiert. Die Notation orientiert sich jeweils an die angegebenen Quellen, um weiterführende Recherchen zu erleichtern.

\section{Lateinische Symbole}

$\mathbf{0}_{x}, \mathbf{0}_{x \times y}$
$a$
$A$
$A_{\text {cat }}, A_{\text {cs }}, A_{\text {geo }}$

\section{$\mathbf{A}, \mathbf{F}$}

$\mathbf{A}_{1}$

$B_{1}, B_{2}, B_{3}$

B

$\mathbf{B}_{1}$

c

c

$\mathbf{C}_{1}$

E

$f$

f

F

$G_{\mathrm{w}}, G_{\mathrm{d}}$

$\mathbf{G}_{\mathrm{c}}$

$H$

$\mathbf{I}_{x}, \mathbf{I}_{x \times y}$

$J$

$k$

$k_{\mathrm{c}}$

$l_{\mathrm{a}}, l_{\mathrm{m}}$

$L$

$\dot{m}$

$M$

$n$

Nullmatrix der Dimension $x \times x$ bzw. $x \times y$

Systemeigenwert

Präexponentieller Faktor in der Arrhenius-Gleichung

Katalytisch wirksame Fläche, Querschnittsfläche des Trägerkörpers, Geometrische Oberfläche des Trägerkörpers

Systemmatrizen in der differential-algebraischen Modelldarstellung

Systemmatrix des linearisierten Zustandsraummodells

Zusammengefasste Parameter, siehe Gleichungen (3.20) und (3.21)

Eingangsmatrix in der differential-algebraischen Modelldarstellung

Eingangsmatrix des linearisierten Zustandsraummodells

Konzentration in der Gasphase

Vektor mit Konzentrationen in der Gasphase

Ausgangsmatrix des linearisierten Zustandsraummodells

Aktivierungsenergie einer Reaktion

Gewichtsfunktion

Vektor mit Gewichtsfunktionen

IMC-Filter

Führungs- bzw. Störübertragungsfunktion des Lambdaregelkreises

Matrix zur Berechnung der Gaskonzentrationen in einer Modellzelle

Enthalpie

Einheitsmatrix der Dimension $x \times x$ bzw. $x \times y$

Gütemaß des LQ-Reglers und der NMPC

Geschwindigkeitskonstante einer Reaktion, Verstärkung

Massentransportkoeffizient

Additive bzw. multiplikative Modellunsicherheit

Sauerstoffspeicherkapazität

Abgasmassenstrom

Motorlastmoment, molare Masse

Motordrehzahl 


$\begin{array}{ll}n_{\mathrm{c}}, n_{\mathrm{g}}, n_{\mathrm{s}} & \text { Anzahl der Modellzellen, der Gas- und der Oberflächenspezies } \\ p_{\mathrm{exh}} & \text { Abgasdruck } \\ P & \text { Zu regelnde Strecke } \\ \tilde{P}, \tilde{P}_{\lambda} & \text { Übertragungsfunktion des Motormodells bzw. der Lambdasonde } \\ Q & \text { Übertragungsfunktion des Lambdareglers } \\ \mathbf{Q} & \text { Matrix zur Gewichtung der Sauerstofffüllstände im Gütemaß } J \\ r & \text { Reaktionsgeschwindigkeit } \\ \mathrm{R} & \text { Universelle Gaskonstante } \\ R & \text { Gewichtungsfaktor der Stellgröße im Gütemaß } J \\ \dot{S} & \text { Reaktionsquellterm } \\ S & \text { Entropie } \\ S_{x, p}^{\text {rel }} & \text { Relative Sensitivität des Parameters } p \text { bezüglich der Größe } x \\ t_{\mathrm{e}} & \text { Kraftstoffeinspritzzeit } \\ T_{\mathrm{g}}, T_{\mathrm{s}} & \text { Temperatur in der Gasphase bzw. des Trägerkörpers } \\ U_{\mathrm{su}} & \text { Spannung der Sprung-Lambdasonde hinter dem Katalysator } \\ V & \text { Ansatzfunktion für eine Ljapunov-Funktion } \\ w & \text { Komplexes Gewicht beim IMC-Reglerentwurf } \\ x & \text { Konzentration im Washcoat, Atomanzahl } \\ \mathbf{x} & \text { Vektor mit Konzentrationen im Washcoat } \\ y & \text { Ausgang des Systemmodells, Atomanzahl } \\ z & \text { Axiale Koordinate, Atomanzahl }\end{array}$

\section{Griechische Symbole}

$\eta \quad$ Konvertierungsrate

$\Theta \quad$ Sauerstofffüllstand bzw. Belegungsgrad einer Oberflächenspezies

$\Theta \quad$ Vektor mit den Sauerstofffüllständen aller Modellzellen bzw. mit den Belegungsgraden aller Oberflächenspezies in einer Zelle des Modells

$\begin{array}{ll}\kappa_{\mathrm{B}}, \kappa_{\mathrm{C}} & \text { Steuerbarkeits- bzw. Beobachtbarkeitsmaß nach Lükel/Müller } \\ \lambda & \text { Lambdawert } \\ \lambda_{\mathrm{d}} & \text { Lambdaabweichung im Motorabgas } \\ \lambda_{\mathrm{m}} & \text { Lambdawert im Motorabgas } \\ \lambda_{\mathrm{u}} & \text { Stellgröße des Lambdareglers } \\ \lambda_{\mathrm{w}} & \text { Sollwert des Lambdaregelkreises } \\ v & \text { Vorzeichenbehafteter stöchiometrischer Koeffizient } \\ \tau & \text { Abtastzeit } \\ \tau_{(\cdot)} & \text { Zeitkonstante } \\ \tau_{\mathrm{d}} & \text { Totzeit des Motormodells } \\ \tau_{\mathrm{m}} & \text { Zeitkonstante des Motormodells } \\ \omega & \text { Kreisfrequenz }\end{array}$




\section{Indizes}

$i$

$(\cdot)^{\text {in }},(\cdot)^{\text {out }}$

$j$

$(\cdot)_{(j)}$

$k$

lsu, su

$S$

$(\cdot)$

$(\cdot)$

$(\cdot)_{\infty}$

Gasspezies, lokaler Index

Größe am Eingang bzw. am Ausgang des Katalysators

Nummer der Reaktion bzw. der Teilreaktion

Mit der Reaktion $j$ assoziierte Gas- bzw. Oberflächenspezies

Nummer einer Zelle des nach dem Ort diskretisierten Modells

Lambdasonde vor bzw. hinter dem Katalysator

Oberflächenspezies

Mittelwert, konjugiert komplex, obere Schranke der Modellunsicherheit

Schätzwert

Stationärer Wert

\section{Chemische Spezies}

$\mathrm{Ce}_{2} \mathrm{O}_{3}, \mathrm{Ce}_{2} \mathrm{O}_{4} \quad$ Ceroxid in reduzierter bzw. oxidierter Form

$\mathrm{CO} \quad$ Kohlenmonoxid

$\mathrm{CO}_{2} \quad$ Kohlenstoffdioxid

$\mathrm{H}_{2} \quad$ Wasserstoff

$\mathrm{H}_{2} \mathrm{O} \quad$ Wasser

$\mathrm{N}_{2} \quad$ Stickstoff

NO Stickstoffmonoxid

$\mathrm{O}_{2} \quad$ Sauerstoff

\section{Abkürzungen}

$\begin{array}{ll}\text { cpsi } & \text { Cells Per Square Inch } \\ \text { E/A } & \text { exakte Ein-/Ausgangslinearisierung } \\ \text { ECU } & \text { Electronic Control Unit } \\ \text { FTP 75 } & \text { Federal Test Procedure 75 } \\ \text { IMC } & \text { Internal Model Control } \\ \text { LQ } & \text { Linear Quadratisch } \\ \text { SCR } & \text { Selective Catalytic Reduction }\end{array}$




\section{Zusammenfassung}

Zur effizienten Abgasnachbehandlung bei Benzinmotoren hat sich seit mehr als drei Jahrzehnten der Drei-Wege-Katalysator bewährt. Die physikalische Modellbildung des Katalysators führt auf nichtlineare, partielle Differentialgleichungen. Je nach Detaillierungsgrad der physikochemischen Modellbildung können die Katalysatormodelle sehr komplex ausfallen und diese Komplexität erschwert die systematische systemtheoretische Analyse des Katalysators. Daher beschränkt sich der Reglerentwurf in der Literatur auf recht einfache Katalysatormodelle und meist lineare Regelungskonzepte. Der Umstand, dass die bekannten Katalysatormodelle nicht in Form von Zustandsraummodellen vorliegen, erschwert den Einsatz der bewährten Methoden der Regelungstechnik weiter.

In der vorliegenden Arbeit wird ein neuer Ansatz zur Erstellung nichtlinearer Zustandsraummodelle aus vereinfachten physikochemischen Katalysatormodellen präsentiert. Als Ausgangspunkt dient ein Modell der Sauerstoffspeicherung durch Ceroxid, das komplexer als die bisher zum Reglerentwurf eingesetzten Modelle ist. Auf dem Weg zur Herleitung des Zustandsraummodells werden hier die sogenannten Gewichtsfunktionen definiert. Die Gewichtsfunktionen ermöglichen eine kompakte und intuitive Beschreibung des Katalysatormodells und ihre Erweiterung auf andere Reaktionsschemata wird detailliert untersucht.

Die Modelldarstellung im Zustandsraum erlaubt die analytische Systemanalyse, wodurch z. B. die asymptotische Stabilität des Katalysators nachgewiesen und das nichtlineare Modell analytisch linearisiert wird. Ein wesentlicher Aspekt der Systemanalyse ist die Berechnung von Strukturmaßen, die den quantitativen Vergleich verschiedener Katalysatoren ermöglichen und so den Materialentwicklungsprozess unterstützen können. Bevor das Modell in der Praxis eingesetzt wird, werden zuerst seine Parameter optimiert. Anhand von analytischen Sensitivitätsanalysen werden die für das Ein-/Ausgangsverhalten wichtigen Modellparameter identifiziert und anschließend mit Messdaten von einem Laborprüfstand optimiert.

Die Modellbildung und die Systemanalyse liefern tiefe Einblicke in die Systemstruktur und das Systemverhalten, die beim Erstellen eines Regelungskonzeptes für den Katalysator nützlich sind. Aufgrund der schlechten Beobachtbarkeit des Katalysators wird ein Open-Loop-Beobachter eingesetzt. Das stark nichtlineare Systemverhalten motiviert des Weiteren den Entwurf von nichtlinearen Reglern. Die Arbeit beginnt mit dem Entwurf eines unterlagerten Lambdaregelkreises und schließt mit dem Entwurf und dem Vergleich von vier dem Lambdaregelkreis überlagerten Emissionsreglern. Zwei Emissionsregler werden mittels exakter Ein-/Ausgangslinearisierung entworfen, einer wird in Form einer nichtlinearen modellprädiktiven Regelung ausgeführt und die linearen Regler sind durch einen LQ-Regler vertreten. Der LQ-Regler und einer der mittels exakter Ein/Ausgangslinearisierung entworfenen Regler werden am Laborprüfstand implementiert und einer klassischen Lambda-Eins-Regelstrategie gegenübergestellt. 


\section{Einführung}

Diese Arbeit befasst sich mit Aspekten der Modellbildung, der Systemanalyse und der Regelung von Drei-Wege-Katalysatoren zur Abgasnachbehandlung bei Fahrzeugen mit Benzinmotoren. Dieses Kapitel stellt eine Einführung in das Gebiet der Abgasnachbehandlung dar und beginnt mit einem kurzen Überblick über die Schadstoffe im Motorabgas, die Entwicklungsgeschichte des Drei-WegeKatalysators, die Hauptkomponenten jedes Abgasnachbehandlungssystems und den Stand der Technik. Im zweiten Abschnitt werden die Problemstellung und die daraus resultierenden Ziele dieser Arbeit erläutert. Das Kapitel schließt mit der Erläuterung der Struktur dieser Arbeit ab.

\subsection{Einleitung}

\subsubsection{Schadstoffe im Motorabgas}

Seit seiner Erfindung vor 130 Jahren hat sich der Benzinmotor als Antrieb für Fahrzeuge und Maschinen etabliert. Im Motor wird ein Luft-Kraftstoff-Gemisch verbrannt, wodurch die im Kraftstoff gespeicherte chemische Energie in mechanische Arbeit umgewandelt wird [25]. Die Hauptenergieträger im Kraftstoff sind die Elemente Kohlenstoff $(\mathrm{C})$ und Wasserstoff $\left(\mathrm{H}_{2}\right)$, die in Form von verschiedenen chemischen Verbindungen im Kraftstoff enthalten sind. Bei der Verbrennung des Luft-Kraftstoff-Gemisches unter idealen Bedingungen entstehen nur die drei Produkte Kohlenstoffdioxid $\left(\mathrm{CO}_{2}\right)$, Wasser $\left(\mathrm{H}_{2} \mathrm{O}\right)$ und Stickstoff $\left(\mathrm{N}_{2}\right)$ [75]. Komplexe Vorgänge bei der Aufbereitung und beim Transport des Luft-Kraftstoff-Gemisches wie die Wandfilmbildung bei Motoren mit Saugrohreinspritzung, Verbrennungsanomalien wie Glüh- und Selbstzündungen und unerwünschte Komponenten im Kraftstoff wie Schwefel (S) sorgen jedoch in der Realität für nicht ideale Prozessrandbedingungen [25, 75]. Infolgedessen enthält das Motorabgas neben den oben erwähnten ungiftigen Verbrennungsprodukten noch unerwünschte Nebenprodukte, die zu den Schadstoffen zählen. Als Schadstoffe werden alle luftfremden Stoffe bezeichnet, die zu einer Störung des natürlichen und des biologischen Gleichgewichts führen können [25].

Die Hauptschadstoffe im Abgas von Benzinmotoren sind Kohlenmonoxid (CO), Kohlenwasserstoffe (HC), Stickoxide $\left(\mathrm{NO}_{\mathrm{x}}\right)$ und Rußpartikel. Diese Schadstoffe werden als limitierte Schadstoffe bezeichnet, da ihre Menge im Autoabgas durch gesetzliche Abgasnormen begrenzt ist. Im rohen Motorabgas sind noch weitere Schadstoffe wie Schwefeldioxid $\left(\mathrm{SO}_{2}\right)$ oder Ammoniak $\left(\mathrm{NH}_{3}\right)$ vorhanden, allerdings in Mengen, die um den Faktor $10^{-3}$ bis $10^{-6}$ kleiner sind als die Mengen der limitierten Schadstoffe. Daher sind ihre Mengen durch die Abgasnormen nicht explizit begrenzt. Allerdings dürfen bei der Abgasnachbehandlung nicht größere Mengen der nicht limi- 


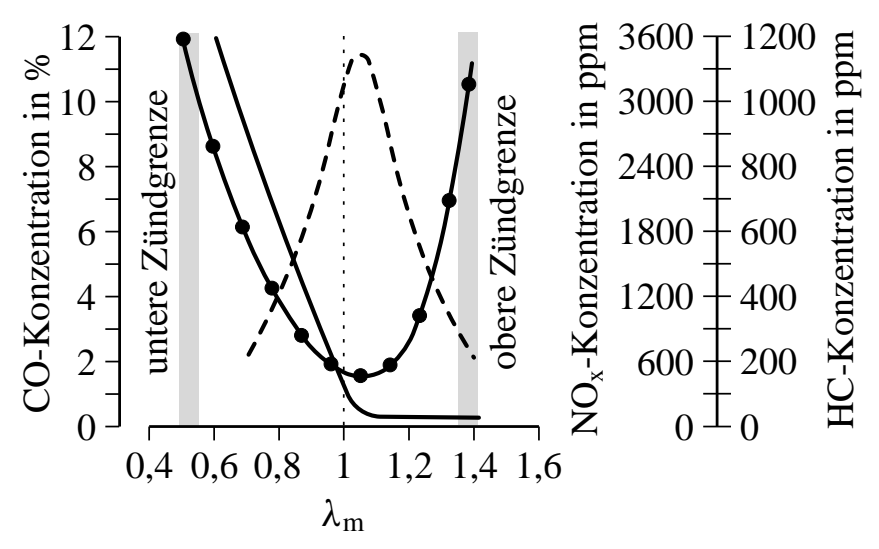

Bild 1.1: Konzentrationen der limitierten Schadstoffe im Motorabgas in Abhängigkeit vom Lambdawert, aus [25]: $\mathrm{CO}(-), \mathrm{NO}_{\mathrm{x}}(---\cdot)$ und $\mathrm{HC}(-\bullet)$

tierten Schadstoffe entstehen [25]. Obwohl $\mathrm{CO}_{2}$ an sich kein Schadstoff ist, kann es in Europa zu den limitierten Schadstoffen gezählt werden, da es als Treibhausgas zur Erderwärmung beiträgt [93, 106]. Da $\mathrm{CO}_{2}$ eines der Hauptprodukte der Verbrennung ist, können seine Emissionen nur durch eine Reduktion des Kraftstoffverbrauchs reduziert werden. In der Europäischen Union wird eine Verringerung der $\mathrm{CO}_{2}$-Emissionen von neuen Personenkraftfahrzeugen im Mittel auf $95 \mathrm{~g} / \mathrm{km}$ bis 2020 angestrebt [28]. So sollen z. B. alle neuen Fahrzeuge am Verkaufsort mit Informationen zum Kraftstoffverbrauch und den $\mathrm{CO}_{2}$-Emissionen versehen werden, damit die Entscheidung der Käufer zugunsten von Fahrzeugen mit niedrigem Verbrauch erleichtert wird [27]. In [36] ist ein Überblick über die natürlichen und vom Menschen verursachten Schadstoffemissionen zu finden.

Die Menge an Sauerstoff $\left(\mathrm{O}_{2}\right)$, die zur vollständigen Verbrennung von 1 mol Kraftstoff benötigt wird, wird stöchiometrischer Sauerstoffbedarf genannt und kann aus der folgenden Reaktionsgleichung unter der Kenntnis der chemischen Zusammensetzung des Kraftstoffs $\mathrm{C}_{x} \mathrm{H}_{y} \mathrm{O}_{z}$ berechnet werden:

$$
\mathrm{C}_{x} \mathrm{H}_{y} \mathrm{O}_{z}+\left(x+\frac{y}{4}-\frac{z}{2}\right) \mathrm{O}_{2} \rightarrow x \mathrm{CO}_{2}+\frac{y}{2} \mathrm{H}_{2} \mathrm{O} .
$$

Der Sauerstoff für die Verbrennung wird der Umgebungsluft entzogen. Unter der Kenntnis der chemischen Zusammensetzung der Luft, die etwa $78 \% \mathrm{~N}_{2}$ und $21 \% \mathrm{O}_{2}$ enthält, kann die stöchiometrische Luftmenge $L_{\text {st }}$ bestimmt werden. Bei der Verbrennung im realen Motor steht jedoch in der Regel nicht die stöchiometrische Luftmenge zur Verfügung. Die Kennzahl $\lambda$ bezieht die tatsächlich zugeführte Luftmenge $L$ auf die stöchiometrische Luftmenge $L_{\mathrm{st}}$

$$
\lambda=\frac{L}{L_{\mathrm{st}}}
$$

und ist eine wichtige Kenngröße der Verbrennung. Bei Luftüberschuss $(\lambda>1)$ wird das LuftKraftstoff-Gemisch als „mageres Gemisch“ und bei Luftmangel $(\lambda<1)$ als „fettes Gemisch“ bezeichnet. Wird der Lambdawert über die Atombilanz berechnet, so ist er stationär betrachtet eine Erhaltungsgröße und deshalb vor und hinter dem Motor gleich [83]. In der Praxis wird der Lambdawert nicht anhand der Atombilanz, sondern anhand der zugeführten Kraftstoff- und Luftmasse ermittelt [106]. 
In Bild 1.1 sind die Konzentrationen der limitierten Schadstoffe im Motorabgas in Abhängigkeit vom Lambdawert dargestellt. Bei unvollständiger Verbrennung unter Luftmangel steigen die COund HC-Emissionen an, während bei hohen Verbrennungstemperaturen und einem Luftüberschuss die NO-Emissionen ansteigen. $\mathrm{CO}$ entsteht als Zwischenprodukt der $\mathrm{CO}_{2}$-Bildung und $\mathrm{HC}$ entstehen vor allem bei unvollständiger Verbrennung des Kraftstoffs aber auch aus dem Schmierfilm der Zylinder. Unter dem Begriff $\mathrm{NO}_{\mathrm{x}}$ werden mehrere Oxide von $\mathrm{N}_{2}$ zusammengefasst. Dabei reagieren $\mathrm{N}_{2}$ und $\mathrm{O}_{2}$ aus der Luft vor allem zu Stickstoffmonoxid (NO) und in kleineren Mengen weiter zu Stickstoffdioxid $\left(\mathrm{NO}_{2}\right)[25,41,75,106]$. Unter der Einwirkung der UV-Strahlung der Sonne reagieren $\mathrm{CO}, \mathrm{HC}$ und $\mathrm{NO}_{\mathrm{x}}$ weiter zu Ozon $\left(\mathrm{O}_{3}\right)$ und weiteren Photooxidanten, die die Hauptbestandsteile von photochemischem Smog darstellen und die wie die limitierten und die nicht limitierten Schadstoffe selbst gesundheitsschädlich sind [37, 101].

\subsubsection{Anfänge der Abgasnachbehandlung}

Am Anfang der Entwicklung des Automobils lag der Schwerpunkt mehr auf der reinen Funktionsund Leistungsfähigkeit und weniger auf der Reduktion der Umweltbelastung und des Kraftstoffverbrauchs [25]. Verbunden mit dem Erfolg des Automobils stiegen auch die Schadstoffemissionen in der Luft und in den 1940er- und 1950er-Jahren verschlechterte sich die Luftqualität in einigen Großstädten wie Los Angeles merklich [101, 105]. Neben der industriellen Verbrennung von fossilen Brennstoffen waren die Fahrzeugmotoren eine große Quelle für die Ausgangssubstanzen des photochemischen Smogs. Nach 1945 wurden die Umweltprobleme zunehmend als gesundheitsschädlich erkannt und seit Ende der 1950er-Jahre änderte sich die öffentliche Wahrnehmung in Richtung einer allgemeinen ,ökologischen“ Sichtweise. Es dauerte jedoch noch bis in die 1960er-Jahre hinein, bis die Fachwelt in den USA die Gesundheitsbedenken akzeptierte und im Jahr 1965 Grenzwerte für die Abgasemissionen von Fahrzeugen mit dem „Motor Vehicle Air Pollution Control Act“ festgelegt wurden [101]. Die umfassenden Änderungen im „Clean Air Act“ im Jahr 1970 forderten eine Reduzierung des Schadstoffausstoßes von Neuwagen um $90 \%$ bis 1975 [23]. In Japan wurden Abgasnormen für Kraftfahrzeuge vom Ministerium für Internationalen Handel und Industrie am Beispiel der Abgasnormen in den USA festgelegt. Zunächst behaupteten die Fahrzeughersteller in beiden Ländern, dass die Grenzwerte in den Abgasnormen nicht zu erfüllen seien. Jedoch arbeiteten die japanischen Fahrzeughersteller an der gleichzeitigen Erfüllung der Abgasnormen in beiden Ländern und manche Fahrzeughersteller konnten die Abgasnormen erfüllen, noch bevor sie in Kraft getreten sind. 1978 betrug der Schadstoffausstoß von neuen japanischen Autos nur etwa $10 \%$ von dem im Jahr 1968 und diese Entwicklung erleichterte das Eindringen der japanischen Automobilhersteller in den 1970er-Jahren in den USFahrzeugmarkt [62]. Vom Rat der Europäischen Gemeinschaften wurden 1970 auch in Europa Grenzwerte für den Schadstoffausstoß von Fahrzeugen festgelegt [21]. Diese Grenzwerte wurden in den folgenden Jahren schrittweise verschärft und ab 1993 wurden mit Euro 1 die bekannten Euro-Abgasnormen eingeführt [22]. Ab diesem Zeitpunkt waren die Abgasnormen in Europa mit denen in den USA und in Japan vergleichbar [36]. 


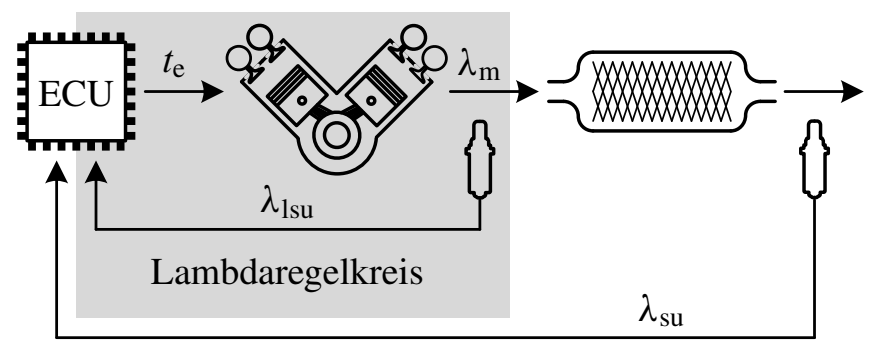

Bild 1.2: Regelkreis zur Abgasnachbehandlung mit Breitband-Lambdasonde vor und SprungLambdasonde hinter dem Katalysator

Innermotorische Maßnahmen zur Reduktion des Schadstoffausstoßes wie die Verbesserung der Gemischaufbereitung und des Verbrennungsprozesses oder der Einsatz einer Abgasrückführung reichten alleine nicht zur Erfüllung der Abgasnormen in den 1970er-Jahren in den USA aus, was zur Entwicklung des Oxidationskatalysators führte. Um genügend Sauerstoff zur Oxidation der unverbrannten Kohlenwasserstoffe und des Kohlenstoffmonoxids zur Verfügung zu stellen, wird im Motorabgas vor dem Katalysator zusätzlich Luft zugeführt. Bis 1975 war es effektiver, die Stickoxide über die im fetten Motorabgas vorhandenen $\mathrm{CO}$ und $\mathrm{HC}$ zu reduzieren, anstatt über einen weiteren Platin/Rhodium-Katalysator, der ab 1976 vor dem Oxidationskatalysator eingesetzt wurde [1]. Ein konzeptioneller Nachteil des Oxidationskatalysators ist, dass die Reduktion der Stickoxide durch das fette Motorabgas mit einem erhöhten Kraftstoffverbrauch verbunden ist. Das Abgasnachbehandlungssystem aus zwei Katalysatoren ist zudem nicht sehr effizient, da im fetten Motorabgas das $\mathrm{NO}$ zum großen Teil in $\mathrm{NH}_{3}$ umgewandelt und im Oxidationskatalysator das $\mathrm{NH}_{3}$ zurück in $\mathrm{NO}$ umgewandelt wird $[92,93]$. Es wurde jedoch schon früh bei der Katalysatorentwicklung festgestellt, dass $\mathrm{CO}, \mathrm{HC}$ und $\mathrm{NO}_{\mathrm{x}}$ gleichzeitig in einem einzigen Katalysator umgesetzt werden können, solange sich der Lambdawert im Motorabgas nah am stöchiometrischen Wert, im sogenannten Lambdafenster, befindet [94]. Dieser Eigenschaft, die drei Hauptschadstoffe gleichzeitig umzusetzen, verdankt der Drei-Wege-Katalysator seinen Namen. Die bis dato verbauten Vergasersysteme konnten allerdings die präzise Kraftstoffdosierung, wie sie zur Einstellung des stöchiometrischen Luft-Kraftstoff-Verhältnisses notwendig ist, nicht leisten. Ab Anfang der 1980er-Jahre waren die zu einer Lambdaregelung notwendigen Sensoren zum Erfassen des Lambdawertes, ausreichend leistungsfähige Mikroprozessoren für die Regelalgorithmen, sowie elektronische Kraftstoffeinspritzung verfügbar [18, 40]. Damit stand dem Einsatz des DreiWege-Katalysators nichts mehr im Wege und er etablierte sich schnell zur effizientesten Form der Abgasnachbehandlung bei Benzinmotoren [81, 105]. Seit 1993 ist der Drei-Wege-Katalysator für alle in der Europäischen Union neu zugelassenen Fahrzeuge mit Benzinmotor gesetzlich vorgeschrieben $[21,36]$.

\subsubsection{Hauptkomponenten des Systems zur Abgasnachbehandlung}

Das Strukturbild des Regelkreises zur Abgasnachbehandlung nach dem heutigen Stand der Technik ist in Bild 1.2 dargestellt. Der Regelkreis enthält den Katalysator, durch den das Motorabgas geleitet wird, eine Breitband-Lambdasonde vor und eine Sprung-Lambdasonde hinter dem Ka- 
a)

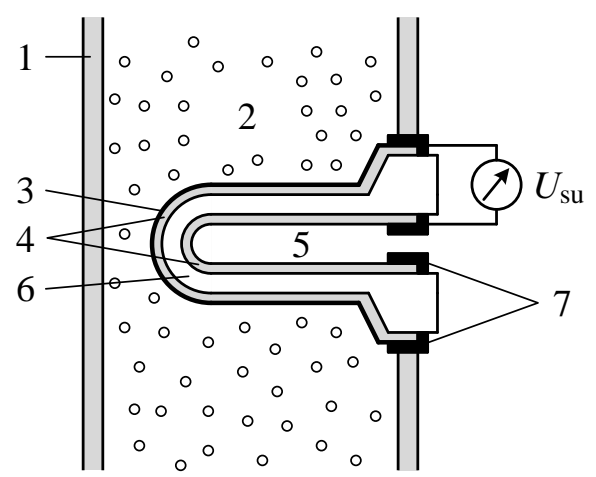

b)

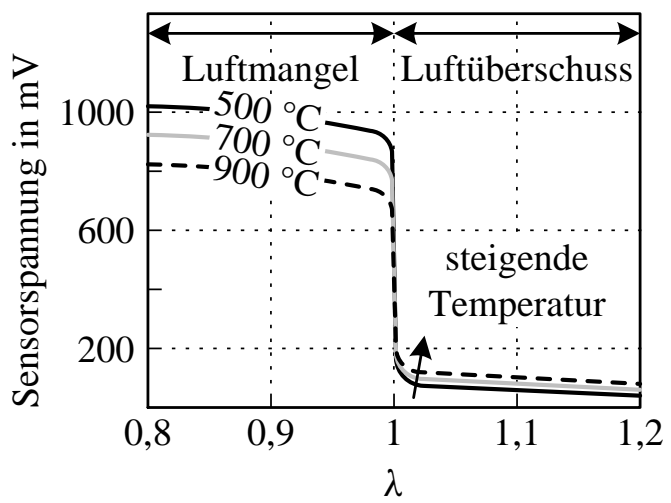

Bild 1.3: a) Prinzipieller Aufbau und b) Kennlinie einer Sprung-Lambdasonde, aus [81]: (1) Abgasrohr, (2) Abgas, (3) Schutzschicht, (4) Elektroden, (5) Referenzluft, (6) $\mathrm{ZrO}_{2}$-Keramik, (7) Kontakte

talysator sowie das Motorsteuergerät (ECU), das die Kraftstoffeinspritzzeit $t_{\mathrm{e}}$ berechnet. In der Praxis wird der Drei-Wege-Katalysator oft in einen motornahen Vorkatalysator und einen Hauptkatalysator aufgeteilt. Der kleine Vorkatalysator kommt schnell auf Betriebstemperatur und kann bereits kurz nach dem Motorstart die Schadstoffemissionen reduzieren. Der Vorkatalysator ist jedoch bei hoher Motorlast einer sehr hohen thermischen Belastung ausgesetzt und muss bezüglich seiner Hochtemperaturstabilität optimiert werden. Die thermische Belastung des Hauptkatalysators ist niedriger und er kann bezüglich der Anspringtemperatur und der Konvertierung der Stickoxide optimiert werden [81]. In dieser Arbeit liegt der Fokus auf der Methodenentwicklung für die Analyse und die Regelung des Drei-Wege-Katalysators, wobei der Einfachheit halber die Konfiguration in Bild 1.2 mit einem Katalysator betrachtet wird.

Sprung-Lambdasonde Die ersten Sensoren zum Erfassen des Lambdawertes im Motorabgas wurden 1976 von der Firma Bosch entwickelt. Diese sogenannten Sprung-Lambdasonden bestehen aus einer gasundurchlässigen $\mathrm{ZrO}_{2}$-Keramik, die das Motorabgas von der Umgebungsluft trennt und ab etwa $350^{\circ} \mathrm{C}$ für Sauerstoffionen durchlässig wird, siehe Bild 1.3a. Je nach Verhältnis der Sauerstoffpartialdrücke auf beiden Seiten der Keramik stellt sich eine Spannung zwischen den auf der Keramik angebrachten Platinelektroden ein. Die Platinelektrode auf der Motorabgasseite wirkt wie ein kleiner Abgaskatalysator und bewirkt, dass das Motorabgas an der Platinelektrode nah an das chemische Gleichgewicht gebracht wird. Die sich einstellende Spannung kann nach der Nernst-Gleichung bestimmt und als Maß für den Sauerstoffgehalt im Motorabgas herangezogen werden $[8,81]$. Die Kennlinie der Sprung-Lambdasonde ist in Bild 1.3b dargestellt. Der Sprung bei $\lambda=1$ resultiert aus der starken Änderung des Sauerstoffpartialdrucks im Motorabgas in diesem Bereich um etwa zehn Größenordnungen. Die Temperatur beeinflusst die Durchlässigkeit der Keramik für Sauerstoffionen und damit die Sensorkennlinie. Der Bereich mit der großen Steigung um $\lambda=1$ weist jedoch eine geringe Temperaturabhängigkeit auf und so kann mit der Sprung-Lambdasonde zuverlässig bestimmt werden, ob das Motorabgas mager oder fett ist. Der Übergang von fett nach mager geschieht bei etwa $450-500 \mathrm{mV}$. 


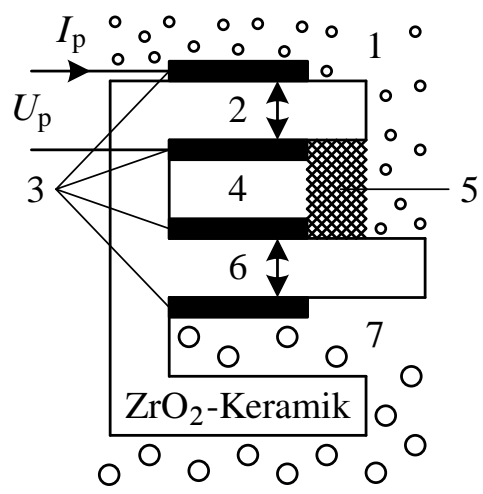

Bild 1.4: Prinzipieller Aufbau einer Breitband-Lambdasonde, aus [106]: (1) Abgas, (2) Pumpzelle, (3) Elektroden, (4) Messkammer, (5) Diffusionsbarriere, (6) Nernst-Zelle, (7) Luftreferenz

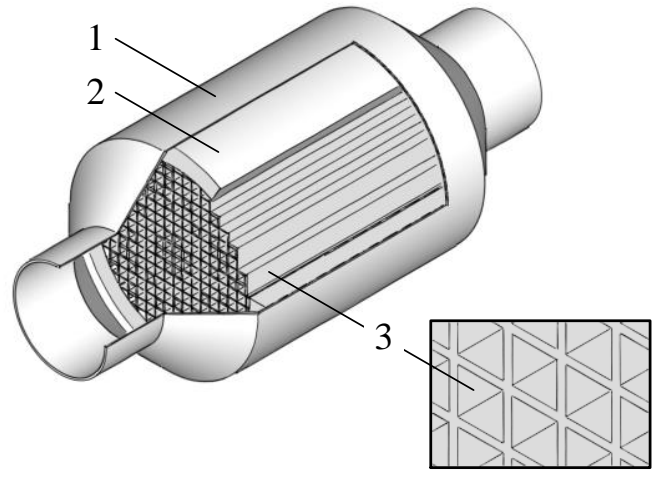

Bild 1.5: Prinzipieller Aufbau des DreiWege-Katalysators, in Anlehnung an [81]: (1) Metallgehäuse, (2) Matte, (3) Trägerkörper

Breitband-Lambdasonde Eine Weiterentwicklung der Sprung-Lambdasonde stellt die seit 1998 verfügbare Breitband-Lambdasonde dar, die den Lambdawert im Bereich von etwa 0,7 bis $\infty$ (Luft mit $21 \%$ Sauerstoffgehalt) kontinuierlich erfassen kann. In Bild 1.4 ist der prinzipielle Aufbau der Breitband-Lambdasonde dargestellt. Diese besteht aus der Nernst-Zelle der Sprung-Lambdasonde und einer weiteren elektrochemischen Zelle, der sogenannten Pumpzelle. Die Nernst-Zelle erfasst nicht den Sauerstoffgehalt des Motorabgases, sondern einer sensorinternen Kammer, die über eine Diffusionsbarriere mit dem Motorabgas verbunden ist. Durch das Anlegen einer Spannung an die Elektroden der Pumpzelle werden Sauerstoffionen durch die Keramik der Pumpzelle transportiert. Mit Hilfe der Nernst-Zelle wird die Pumpspannung so geregelt, dass in der Messkammer $\lambda=1$ herrscht. Der Pumpstrom ist proportional zum Sauerstoffpartialdruck im Motorabgas und kann daher als Maß für den Sauerstoffgehalt herangezogen werden. In $[8,71,81,83]$ sind weiterführende Informationen zum Aufbau und der Funktionsweise der Lambdasonden zu finden.

Drei-Wege-Katalysator Der Abgaskatalysator besteht in der Regel aus einem Metallgehäuse, einem wabenförmigen Trägerkörper und einer dazwischen angebrachten Matte, die zum einen mechanische Erschütterungen dämpft und zum anderen zur Wärmeisolation dient, siehe Bild 1.5 [58, 63]. Der Trägerkörper weist zwecks Erhöhung der spezifischen geometrischen Oberfläche $A_{\text {geo }}$ Kanäle in axialer Richtung auf. Je nach Katalysatortechnologie können sich zwischen 400 und 1200 solcher Kanäle pro Quadratzoll der Stirnfläche befinden. Der Trägerkörper kann aus Keramik, vorzugsweise Cordierit, oder aus dünnen Metallfolien aufgebaut sein. Durch den Einsatz von sehr dünnwandigen Folien kann bei Metallträgern eine hohe Kanalanzahl und damit eine große geometrische Oberfläche pro Volumen erreicht werden und dabei gleichzeitig der Anstieg des Strömungswiderstandes in Grenzen gehalten werden [25, 58, 63].

Auf dem Trägerkörper wird der sogenannte Washcoat aufgetragen. Der Washcoat vergrößert zum einen die spezifische katalytisch wirksame Oberfläche $A_{\text {cat }}$ und dient zum anderen als Trägeroxid für die katalytisch aktiven Edelmetalle und die Sauerstoffspeicherkomponente [16, 25]. Als Trä- 
geroxid wird am häufigsten Aluminiumoxid $\left(\mathrm{Al}_{2} \mathrm{O}_{3}\right)$ eingesetzt, das eine spezifische Oberfläche im Bereich von 100 bis $200 \mathrm{~m}^{2} / \mathrm{g}$ aufweist. Im Vergleich dazu weist der Trägerkörper aus Cordierit allein eine spezifische Oberfläche von etwa $0,7 \mathrm{~m}^{2} / \mathrm{g}$ auf [63]. Neben der Erhöhung der katalytisch wirksamen Oberfläche soll der Washcoat die feine Verteilung der Edelmetalle stabilisieren, eine hohe Resistenz gegenüber Schadstoffen und Phasenumwandlungen und eine gute Haftung auf dem Trägerkörper aufweisen [53].

Als katalytisch aktive Materialien im Drei-Wege-Katalysator kommen heutzutage vor allem die Edelmetalle Palladium (Pd) und Rhodium (Rh), früher auch Platin (Pt), zum Einsatz [14, 46, 93, 105]. Obwohl es an Versuchen nicht mangelte, haben sich andere Materialien nicht durchgesetzt, da nur die Edelmetalle die hohe katalytische Aktivität aufweisen, um die Schadstoffe in der nur wenige Millisekunden langen Verweilzeit im Katalysator zu neutralisieren. Zudem haben die Edelmetalle eine hohe Resistenz gegenüber einer Deaktivierung durch Schwefeloxide $\left(\mathrm{S}_{x} \mathrm{O}_{y}\right)$ und gegenüber einer Deaktivierung bei hohen Temperaturen durch die Trägeroxide des Washcoats.

Im transienten Motorbetrieb kommt es z. B. durch Fehler bei der Kraftstoffeinspritzung zu Abweichungen des Lambdawertes im Motorabgas vom stöchiometrischen Wert. Durch die Fähigkeit des Katalysators, Sauerstoff zu speichern und wieder abzugeben, können kurzzeitige Abweichungen des Lambdawertes überbrückt werden, ohne dass es zu erhöhten Schadstoffemissionen hinter dem Katalysator kommt. Als Sauerstoffspeicherkomponente wird heutzutage Ceroxid $\left(\mathrm{CeO}_{2}\right)$ oder Zirkonoxid $\left(\mathrm{ZrO}_{2}\right)$ eingesetzt, wobei Ceroxid bereits bei der Entwicklung der ersten DreiWege-Katalysatoren eingesetzt wurde [16, 103, 105].

\subsubsection{Stand der Technik}

Ohne Anspruch auf Vollständigkeit wird im Folgenden kurz der Stand der Technik bei der Abgasnachbehandlung von stöchiometrisch betriebenen Benzinmotoren dargestellt. Dies sind vor allem Motoren mit Saugrohreinspritzung. Motoren mit Benzin-Direkteinspritzung und homogenem Luft-Kraftstoff-Gemisch besitzen jedoch im Prinzip das gleiche Abgasnachbehandlungssystem [107].

Im betriebswarmen Zustand und bei einem Lambdawert im Motorabgas im Bereich des Lambdafensters erreichen Drei-Wege-Katalysatoren heutzutage Konvertierungsraten der Schadstoffe von über $99 \%$ [13]. Die mittlere Lambdaabweichung vor dem Katalysator sollte dabei weniger als $0,1 \%$ vom stöchiometrischen Wert betragen [40,49]. Durch die diskrete Arbeitsweise von Hubkolbenmotoren entstehen Verzögerungen und Totzeiten zwischen der Kraftstoffeinspritzung und der Erfassung des Lambdawertes $\lambda_{m}$ im Motorabgas, siehe Bild 1.2. Diese Verzögerungen und diese Totzeiten limitieren die Bandbreite des Lambdaregelkreises, weshalb eine ZweiFreiheitsgrade-Regelkreisstruktur mit einer Vorsteuerung der Kraftstoffeinspritzung und einem Lambdaregler verwendet wird. Die Vorsteuerung wird für die schnelle Kraftstoffdosierung bei transienten Vorgängen benötigt und berechnet anhand der geschätzten angesaugten Luftmenge die benötigte Kraftstoffmenge. Der langsame Lambdaregler kompensiert die Fehler der Vorsteuerung und sorgt für die stationäre Genauigkeit des Lambdaregelkreises [5, 40]. 
Vor der Entwicklung der Breitband-Lambdasonde wurde eine Sprung-Lambdasonde vor dem Katalysator eingesetzt. Aufgrund ihrer Zweipunkt-Charakterisitk ergibt sich in Kombination mit einem PI-Regler eine Dauerschwingung von $\lambda_{\mathrm{m}}$ um den stöchiometrischen Wert mit einer Amplitude von etwa $3 \%[49,106]$. Dieses periodische Befüllen und Entleeren eines Teils des Sauerstoffspeichers des Katalysators verbessert die Schadstoffkonvertierung, da während der Sauerstoffspeicherung die Reduktion von $\mathrm{NO}_{\mathrm{x}}$ und während der Sauerstoffabgabe die Oxidation von $\mathrm{CO}$ und $\mathrm{HC}$ unterstützt wird [106]. Mit der Einführung der Breitband-Lambdasonde wurde es möglich, den Lambdawert im Motorabgas präziser zu regeln und den Sollwert des Lambdaregelkreises abweichend vom stöchiometrischen Wert zu wählen. So kann der Motor gezielt z. B. in der Warmlaufphase etwas magerer und bei hohen Lasten etwas fetter betrieben werden [81]. Mit der Breitband-Lambdasonde ergibt sich kein Grenzzyklus im Lambdaregelkreis. Ein periodisches, partielles Befüllen und Entleeren des Sauerstoffspeichers wird jedoch durch die Addition einer Zwangsanregung zum Sollwert des Lambdaregelkreises erreicht. Obwohl die Lambdaregelung seit 1993 in jedem neu zugelassenen Fahrzeug mit Benzinmotor vorhanden ist, bleiben die Implementierungen der einzelnen Fahrzeughersteller ein gut gehütetes Geheimnis. Nach dem dokumentierten Stand der Technik werden im Lambdaregelkreis PI- oder PID-Regler eingesetzt [4, $6,50,61,65,70,102,112]$.

Die Breitband-Lambdasonde vor dem Katalysator ist dem heißen, unbehandelten Motorabgas ausgesetzt und aufgrund dieser starken Beanspruchung ändern sich ihre Parameter signifikant während des Betriebs [71]. Sie weist z. B. einen nicht konstanten Offset auf, der die Leistungsfähigkeit der Lambdaregelung beeinträchtigt. Um diesen Offset zu kompensieren, wird nach dem heutigen Stand der Technik das Signal einer Sprung-Lambdasonde hinter dem Katalysator genutzt, siehe Bild 1.2. Da die Betriebsbedingungen an dieser Stelle günstiger sind, ist auch das Sensorsignal verlässlicher. In dieser, der Lambdaregelung überlagerten, Trimmregelung kommen langsame PIRegler zum Einsatz [4, 102, 106].

Die meisten Regelungskonzepte nach dem aktuellen Stand der Technik haben gemeinsam, dass das dynamische Verhalten des Katalysators unberücksichtigt bleibt. Van Basshuysen [106] nennt als Stand der Technik ein PII ${ }^{2}$ D Regler, wobei der I $^{2}$-Anteil zur Bilanzierung der Sauerstoffbeladung des Katalysators dient. Es ist allerdings nicht weiter beschrieben, wie der Regler ausgelegt wird. Daher ist davon auszugehen, dass ähnlich wie bei den PI- und PID-Reglern in der Lambdaund Trimmregelung die Auslegung nicht modellbasiert, sondern heuristisch erfolgt. In den letzten Jahren wurden von mehreren Wissenschaftlern Verbesserungen der Regelkreisstruktur und auf Katalysatormodellen basierende Regelungskonzepte vorgeschlagen. Der Stand der Forschung wird jeweils am Anfang der betreffenden Kapitel dieser Arbeit vorgestellt.

Diagnose des Katalysators Die Nutzung von jeweils einer Lambdasonde vor und hinter dem Katalysator ermöglicht des Weiteren die Überwachung der Schadstoffkonvertierung für die OnBoard-Diagnose des Katalysators. Zum Beispiel sind zur Erfüllung der Abgasnorm Euro IV Konvertierungsraten größer als $96 \%$ notwendig und Überschreitungen der Grenzwerte der Abgasnorm um das 1,5-Fache müssen durch die On-Board-Diagnose registriert werden [40, 81]. Da ein Zusammenhang zwischen den Konvertierungsraten und der Sauerstoffspeicherkapazität besteht, 
basieren die Diagnoseverfahren auf der Ermittlung der Sauerstoffspeicherkapazität. Dabei wird entweder versucht, die Sauerstoffspeicherkapazität direkt zu schätzen, oder es wird die Dämpfung einer Lambdaschwingung im Motorabgas durch den Katalysator ausgewertet [81].

\subsection{Problemstellung und Ziele der Arbeit}

Von der Europäischen Kommission wurde 2010 eine Liste mit kritischen Materialien bezüglich ihrer wirtschaftlichen Bedeutung und ihres Versorgungsrisikos aufgestellt. In dieser Liste und auch in ihrer aktualisierten Fassung aus dem Jahr 2014 sind die Edelmetalle Palladium und Rhodium und das Seltenerdmetall Cer enthalten [26]. Mit der „RAW Materials Initiative“ der Europäischen Kommission wurde 2008 eine Strategie zur Sicherstellung der Versorgung der Europäischen Union mit Rohstoffen gestartet. Eine der zentralen Säulen dieser Strategie ist die Erhöhung der Ressourceneffizienz, um den Rohstoffbedarf zu senken und damit auch Abhängigkeiten von Rohstoffimporten zu reduzieren. Diese Strategie wurde 2011 bewertet und es wurden weitere Verbesserungen beschlossen, z. B. beim Handel mit Rohstoffen aus Entwicklungsländern [29]. Etwa $70 \%$ des weltweiten Bedarfs an Palladium und $80 \%$ an Rhodium wurden 2013 für die Herstellung von Katalysatoren für die Abgasreinigung von Benzinmotoren verwendet [46]. Der entsprechende Anteil der Seltenerdmetalle Lanthan und Cer lag 2012 bei etwa 26\% [91]. Auf dem Weltmarkt für Edelmetalle ist ein Defizit zu beobachten und die Nachfrage nach Palladium und Rhodium seitens der Automobilindustrie ist weiter steigend [46]. Aber nicht nur aus wirtschaftlichen und politischen Gründen, sondern auch aus Sicht des Umweltschutzes ist es sinnvoll, den Ressourceneinsatz bei der Herstellung der Katalysatoren zu senken.

Die Ergebnisse in der vorliegenden Arbeit entstanden im Rahmen des Projektes „ReffKat“, das die Entwicklung von ressourceneffizienten Autoabgaskatalysatoren mit deutlich reduziertem Gehalt an Edelmetallen und Seltenerdmetallen zum Ziel hat. Das Projekt mit dem Förderkennzeichen 03X3563 ist Teil der Förderinitiative „MatRessource“ des Bundesministeriums für Bildung und Forschung zur Entwicklung von Materialien für eine ressourceneffiziente Industrie und Gesellschaft. Bei der Materialentwicklung liegt der Schwerpunkt bei der Verbesserung der CeroxidMischoxide, die den Sauerstoffspeicher des Katalysators bilden [13]. Am Projekt beteiligt sind:

- Umicore AG \& Co. KG

- Institut für Technische Chemie und Polymerchemie am Karlsruher Institut für Technologie

- Ernst-Berl-Institut für Technische und Makromolekulare Chemie an der TU Darmstadt

- Fachgebiet Regelungstechnik und Mechatronik an der TU Darmstadt

Das Aufgabengebiet am Fachgebiet Regelungstechnik und Mechatronik umfasst die Entwicklung eines modellbasierten Regelungskonzeptes für den Drei-Wege-Katalysator. 
Wie in Abschnitt 1.1.4 erklärt, erreichen Drei-Wege-Katalysatoren im betriebswarmen Zustand und bei einem Lambdawert im Motorabgas im Bereich des Lambdafensters sehr hohe Konvertierungsraten der Schadstoffe. Abgesehen von den Emissionen beim Kaltstart des Motors treten erhöhte Schadstoffemissionen vor allem im dynamischen Fahrbetrieb auf, wie z. B. bei plötzlichen Beschleunigungsvorgängen. Um diese Effekte besser zu verstehen, ist ein grundlegendes Verständnis für das Regelverhalten des Katalysators und für den Zusammenhang zwischen seinen regelungstechnischen und chemischen Eigenschaften notwendig. Das System aus Verbrennungsmotor und Katalysator ist so komplex, dass die Katalysatorentwicklung und die Parametrierung der heutzutage eingesetzten heuristischen PI-Regler in aufwendigen Motor- und Fahrzeugtests erfolgen. Neben der Materialentwicklung ist ein wichtiges Ziel des Projektes „ReffKat“ die Steigerung der Effizienz des Entwicklungsprozesses. Um dies zu erreichen, wird zum einen ein Teil der Motor- und der Fahrzeugtests durch einfacher durchzuführende und kostengünstigere Messungen an Laborprüfständen ersetzt. Dafür werden neuartige Laborprüfstände aufgebaut, die das reproduzierbare Nachstellen von hochdynamischen Fahrzyklen ermöglichen [13]. Zum anderen wird eine Verringerung des Parametrierungsaufwandes der Regler angestrebt, indem die heuristischen PI-Regler durch modellbasierte Regler ersetzt werden.

Während bei den heuristischen PI-Reglern das dynamische Verhalten des Katalysators weitgehend unberücksichtigt bleibt, wurden in den letzten Jahren auch auf Katalysatormodellen basierende Regelungskonzepte vorgestellt. Es ist in der Katalysatorentwicklung allgemein bekannt, dass der Katalysator nur so gut sein kann wie die Regelung des Lambdawertes im Motorabgas [93]. Die Vorteile, die mit einer modellbasierten Regelung erzielt werden können, hängen ihrerseits von der Güte des zugrundeliegenden Modells ab. Chemiker, die sich mit der Modellierung des Katalysators beschäftigen, arbeiten vor allem daran, das Ein-/Ausgangsverhalten möglichst gut nachzubilden und den Rechenaufwand zur Simulation des Modells zu reduzieren. Bei der physikochemischen Modellierung werden auf der einen Seite physikalische Transportprozesse und auf der anderen Seite chemische Reaktionen betrachtet. Detaillierte physikochemische Modelle können viele chemische Reaktionen und Spezies berücksichtigen und dadurch mathematisch sehr komplex sein. Zum Beispiel berücksichtigen die Reaktionsschemata von Chattarjee [17] und Koop und Deutschmann [52] über 60 Reaktionen und mehr als 30 chemische Spezies. Da die Zeitkonstanten der meisten chemischen Reaktionen deutlich kleiner als die Zeitkonstanten des Massentransports oder der Wärmeleitung sind, können detaillierte Modelle sehr steif sein und sind dadurch schwierig zu simulieren [40]. Der mathematischen Komplexität und der Recheneffizienz geschuldet sind die Modelle oft so implementiert, dass sie, obwohl sie aus einer theoretischen Analyse hervorgehen, für Regelungstechniker als Black-Box-Modelle erscheinen. Das heißt, sie bilden das Ein-/Ausgangsverhalten des Systems ab, lassen aber keine Einblicke in die Systemstruktur zu. Regelungstechniker sind jedoch stark an der Systemstruktur interessiert, insbesondere dann, wenn es um die Anwendung modellbasierter Verfahren zur Systemanalyse und zum Reglerentwurf geht. Regelungstechnische Modelle sollen deshalb möglichst genau aber gleichzeitig auch einfach und transparent sein, um die Anwendung der Analyse- und der Reglersyntheseverfahren handhabbar zu machen, oder überhaupt erst zu ermöglichen. Die Eigenschaft des Katalysators, Sauerstoff aufzunehmen und wieder abzugeben, bestimmt maßgeblich sein dynamisches Verhalten und so zielen regelungstechnische Modelle vor allem auf die Modellierung der Dynamik der 
Sauerstoffspeicherung ab [40]. Die meisten Modelle in der Literatur haben gemeinsam, dass sie nicht in Form von Zustandsraummodellen vorliegen. Dagegen beruhen die gängigen Verfahren zu Systemanalyse und Reglerentwurf von nichtlinearen Systemen auf Zustandsraummodellen. Dieser Umstand beschränkt den modellbasierten Reglerentwurf in der Literatur auf sehr einfache, meist phänomenologische Katalysatormodelle und oft auf lineare Regelungskonzepte. Daher ist das erste Ziel dieser Arbeit die Entwicklung von nichtlinearen Zustandsraummodellen aus vereinfachten physikochemischen Katalysatormodellen.

Das zweite Ziel dieser Arbeit ist die analytische Analyse des Katalysators aus systemtheoretischer Sicht. Ein Katalysatormodell in Zustandsraumdarstellung erlaubt die Nutzung der bewährten Werkzeuge der Regelungstechnik und so sollen Systemkenngrößen berechnet werden, die den quantitativen Vergleich verschiedener Katalysatoren ermöglichen. Sowohl für das Regelverhalten wichtige Eigenschaften wie Stabilität, Steuer- und Beobachtbarkeit als auch systemspezifische Eigenschaften wie die Konvertierungsraten der Schadstoffe oder die Abhängigkeit der Systemnichtlinearitäten von den Modellparametern sollen analytisch und schnell für verschiedene Parametrierungen berechnet werden können. Die Systemanalyse kann helfen, früh im Prozess der Katalysatorentwicklung das Regelverhalten zu prognostizieren. Die Entscheidung für ein physikochemisches Modell ist damit zu begründen, dass im Vergleich zu einem phänomenologischen Modell die Modellparameter eine physikalische Bedeutung besitzen. Damit können durch Sensitivitätsanalysen für das Regelverhalten wichtige Katalysatorparameter aufgespürt werden und bei der Materialentwicklung versucht werden, diese Parameter gezielt zu optimieren und dadurch eventuell weitere Ressourceneinsparungen zu erzielen.

Das dritte Ziel dieser Arbeit ist das Aufstellen eines geeigneten Regelungskonzeptes für den Katalysator. Die Verbesserung der Regelung bietet neben der Entwicklung neuer Materialien eine weitere Möglichkeit, Ressourcen einzusparen. Dabei sollen die Entscheidungen zur Regelkreisstruktur und zum Typ der Regler anhand der Erkenntnisse aus der Systemanalyse getroffen werden. Sowohl lineare als auch nichtlineare Regler sollen entworfen und in der Simulationsumgebung systematisch auf ihre spezifischen Vor- und Nachteile untersucht werden. Die Reglerparametrierung soll vorzugsweise systematisch und modellbasiert erfolgen und so dem Applikateur nur wenige und intuitiv zu wählende Parameter zur Verfügung stellen. Nicht zuletzt soll das Regelungskonzept an einem Laborprüfstand bei der Firma Umicore implementiert und eine Korrelation zwischen dem simulierten und dem gemessenen Katalysatorverhalten aufgezeigt werden.

\subsection{Struktur der Arbeit}

Wenngleich in dieser Arbeit der Schwerpunkt auf der Modellbildung und der Regelung des Katalysators liegt, widmet sich Kapitel 2 zunächst dem Lambdaregelkreis. Der Grund dafür ist, dass beim Entwurf der eigentlichen Katalysatorregelung die Dynamik des unterlagerten Lambdaregelkreises berücksichtigt werden soll. Zuerst werden die wichtigsten Motorkomponenten im Lambdaregelkreis vorgestellt und modelliert. Die etablierte Zwei-Freiheitsgrade-Struktur mit Vorsteuerung der Kraftstoffeinspritzung und Lambdaregler wird beibehalten und die Betrachtungen 
werden sich auf den Entwurf des Lambdareglers konzentrieren. Für den Lambdaregler wird die Internal Model Control (IMC)-Struktur gewählt, da sie Vorteile sowohl beim Entwurf als auch bei der Implementierung des Reglers bietet. Im Gegensatz zu bekannten IMC-Lambdareglern wird der Regler diskret entworfen und adaptiv implementiert, sodass seine Parameter während der Laufzeit an den Motorarbeitspunkt angepasst werden können. Es werden Betrachtungen bezüglich der gewünschten und der erreichbaren Bandbreite des Lambdaregelkreises angestellt.

Kapitel 3 beschäftigt sich mit der Modellbildung des Drei-Wege-Katalysators. Am Anfang wird die grundsätzliche Vorgehensweise bei der physikalischen Modellbildung vorgestellt. Die dabei getroffenen vereinfachenden Annahmen werden diskutiert. Die Modellbildung orientiert sich dabei an Möller u. a. [64, 65] und ihren Arbeiten wird auch das hier verwendete Reaktionsschema zur Sauerstoffspeicherung durch Ceroxid entnommen. Das Ziel der Modellbildung ist ein $\mathrm{Zu}-$ standsraummodell des Katalysators und auf dem Weg zu seiner Herleitung werden hier die sogenannten Gewichtsfunktionen definiert. Diese wenigen Gewichtsfunktionen ermöglichen eine kompakte und intuitive Beschreibung des Katalysatormodells und ermöglichen die vorteilhafte Ausnutzung der vorhandenen Systemstruktur. Ihre Erweiterung auf andere als das hier eingesetzte Reaktionsschema wird untersucht und es werden beispielhaft zwei andere Reaktionsschemata durch Gewichtsfunktionen dargestellt.

Das aus der Modellbildung hervorgehende nichtlineare Zustandsraummodell des Katalysators eröffnet die Möglichkeit, die bewährten Methoden der Regelungstechnik zur Systemanalyse einzusetzen und dies ist der Gegenstand von Kapitel 4. Die Gewichtsfunktionen können als eine Schnittstelle zwischen der Regelungstechnik und der Chemie angesehen werden und werden zuerst kurz aus diesen zwei Blickwinkeln analysiert. Anschließend wird direkt am nichtlinearen Modell die Stabilität zum einen anschaulich durch das Systemvektorfeld gezeigt und zum anderen durch die Direkte Methode von Ljapunov analytisch nachgewiesen. Für die weiteren Analysen wird das nichtlineare Zustandsraummodell analytisch linearisiert. Eine Betrachtung der Verläufe der stationären Arbeitspunkte und der Zeitkonstanten in Abhängigkeit vom Lambdawert oder vom Sauerstofffüllstand gibt Auskunft über die Stärke der Systemnichtlinearitäten. Wesentlicher Aspekt der Systemanalyse ist die Berechnung von Strukturmaßen, die den quantitativen Vergleich verschiedener Katalysatoren erlauben. Abschließend wird der Einfluss der Temperatur und des Motorabgasmassenstroms auf die Systemeigenschaften untersucht.

Am Projekt „ReffKat“ waren mehrere Projektpartner aus dem Bereich der Chemie beteiligt, welche die Parameter des Katalysatormodells zur Verfügung stellten. Da sich jedoch mit der Alterung des Katalysators die Modellparameter und insbesondere seine Sauerstoffspeicherkapazität ändern, wird in Kapitel 5 die Identifikation der Modellparameter angesprochen. Das Ziel ist eine schnelle Nachoptimierung der von den Projektpartnern ermittelten Parameter nur durch die Nutzung der Signale der Lambdasonden. Der Parameteroptimierung geht eine analytische Sensitivitätsanalyse voraus, um die Parameter zu finden, die am stärksten das Ein-/Ausgangsverhalten des Katalysators beeinflussen. Anschließend werden geeignete Signalverläufe zur Parameteridentifikation festgelegt und Messungen am Laborprüfstand durchgeführt. Die eigentliche Optimierung wird wie von Kiwic u. a. [50, 51] mit einem genetischen Algorithmus durchgeführt. 
Kapitel 6 beschäftigt sich mit dem dritten Schwerpunkt dieser Arbeit, nämlich mit der Erstellung eines Regelungskonzeptes für den Katalysator. Zuerst wird basierend auf den Ergebnissen der Systemanalyse eine geeignete Regelkreisstruktur zum Erreichen der gestellten Regelziele vorgeschlagen. Anschließend werden lineare und nichtlineare Emissionsregler entworfen und in der Simulationsumgebung implementiert. Anhand von Simulationen werden die entworfenen Regler miteinander verglichen und ihre spezifischen Vor- und Nachteile diskutiert. Es wird ein linearer Emissionsregler als LQ-Regler entworfen und die Gewichtungsmatrix der Modellzustände in seinem Gütemaß modellbasiert gewählt. Diese modellbasierte Wahl steht im Gegensatz zu der heuristischen Vorgehensweise in der Literatur. Das Gütemaß des LQ-Reglers wird auch bei der Implementierung einer nichtlinearen modellprädiktiven Regelung eingesetzt, die als eine nicht echtzeitfähige Referenz für die anderen Regler entworfen wird. Es werden weiterhin zwei nichtlineare Regler mittels exakter Ein-/Ausgangslinearisierung entworfen. Der LQ-Regler und einer der mittels exakter Ein-/Ausgangslinearisierung entworfenen Regler wurden am Laborprüfstand bei der Firma Umicore implementiert. Abschließend werden Messdaten zu drei Testszenarien gezeigt und diskutiert.

Die Arbeit schließt mit einer Zusammenfassung und einem Ausblick in Kapitel 7. 


\section{Der Lambdaregelkreis}

Am Anfang dieses Kapitels werden kurz einige Ansätze aus der Literatur zur Verbesserung der nach dem Stand der Technik eingesetzten heuristischen PI- und PID-Regler im Lambdaregelkreis umrissen. Anschließend werden die wichtigsten Motorkomponenten im Lambdaregelkreis erläutert und modelliert. Zur Regelung des Lambdawertes im Motorabgas wird die etablierte Zwei-Freiheitsgrade-Struktur mit Vorsteuerung und Lambdaregler beibehalten, wobei sich die Ausführungen auf den Lambdaregler fokussieren. Für den Lambdaregler wird die IMC-Struktur gewählt, da sie Vorteile sowohl beim Entwurf als auch bei der Implementierung des Reglers bietet. Im Gegensatz zu bekannten IMCLambdareglern wird der Regler diskret entworfen und so implementiert, dass seine Parameter am Motorarbeitspunkt adaptiert werden können. Es werden Betrachtungen bezüglich der gewünschten und der erreichbaren Bandbreite des Lambdaregelkreises angestellt.

Eine Voraussetzung für die Verbesserung der nach dem Stand der Technik eingesetzten heuristischen Regler durch modellbasierte Regler stellen Modelle des Motors dar. Von Interesse ist dabei der Transport der Luft und des Kraftstoffs bis in die Brennräume des Motors und von dort aus der Transport der Abgase bis hin zur Lambdasonde vor dem Katalysator. Guzzella u. a. [39] verwenden ein Motormodell, das den Luft- und den Kraftstoffpfad mit dem Effekt der Wandfilmbildung berücksichtigt. Die Wandfilmbildung ist relevant bei Motoren mit Saugrohreinspritzung und wird im nächsten Abschnitt erläutert. Die Modelle von Inagaki u. a. [43], Kako u. a. [48], Yildiz u. a. [112] sowie [104] berücksichtigen nur den Kraftstoffpfad. Balenovic [5], Postma und Nagamune [78], Roduner u. a. [85], Rupp und Guzzella [86] und Shafai [90] betrachten dahingegen den Luft- und den Kraftstoffpfad zusammen und modellieren die Dynamik des Lambdawertes durch ein $\mathrm{PT}_{1}$-Glied mit Totzeit. Dieses lineare Modell ist weit verbreitet und zeigt sich als ausreichend genau, wenn eine Zwei-Freiheitsgrade-Struktur verwendet wird und die Vorsteuerung der Kraftstoffeinspritzung die Nichtlinearitäten im Lambdaregelkreis weitgehend kompensiert. Die Dynamik der Lambdasonde wird im Allgemeinen durch ein weiteres $\mathrm{PT}_{1}$-Glied mit Totzeit modelliert, siehe die Arbeiten von Balenovic, Rupp und Guzzella sowie Yildiz u. a.

Es werden weiterhin PI- und PID-Regler für die Lambdaregelung vorgeschlagen. Zum Beispiel entwerfen Fiengo u. a. [30] ein PI-Regler mit Anti-Windup und in [33] wird ein diskreter PIDRegler entworfen, wobei dort kein Motormodell zugrunde gelegt wird.

Ein wichtiger Aspekt beim Entwurf des Lambdareglers ist die Berücksichtigung der Totzeit des Motors, da sie die Bandbreite des Lambdaregelkreises stark begrenzt. Eine Möglichkeit hierfür stellt die IMC-Struktur dar. Ähnlich wie beim Smith-Prädiktor wird dabei der Reglerentwurf nur für den minimalphasigen Teil der zu regelnden Strecke durchgeführt. Die IMC-Struktur wird z. B. 
von Balenovic, Inagaki u. a., Rupp und Guzzella sowie Yildiz u. a. eingesetzt. Da sie ein explizites Modell der Strecke enthält, kann sie gut als adaptive Regelung ausgelegt werden, was Rupp und Guzzella, Inagaki u. a. sowie Yildiz u. a. auch machen. Zum Beispiel ist die Adaption des Modells der Lambdasonde wichtig, da sich ihr dynamisches Verhalten infolge der Alterung ändert.

Weitere Ansätze für die Lambdaregelung sind unter anderem der Reglerentwurf mittels Optimierung der $\mathrm{H}_{\infty}$-Norm von Roduner u. a. und Shafai, die modellprädiktiven Regler von Muske u. a. [68] und Kako u. a., die analytisch berechnet werden können und keine Optimierung zur Laufzeit benötigen, sowie der schaltende LPV-Regler von Postma und Nagamune. Postma und Nagamune modellieren den Motor als ein lineares, parameterveränderliches (LPV)-System, teilen den von Motorlast und Motordrehzahl aufgespannte Arbeitsbereich in Teilbereiche auf, entwerfen für jeden Teilbereich einen LPV-Regler und schalten je nach aktuellem Arbeitspunkt zwischen den Reglern um. Guzzella u. a. sowie [12] schlagen nichtlineare Regelungskonzepte vor, die auf der Zustandslinearisierung des Luft- und des Kraftstoffpfades basieren. Da die Stelleingriffe der Lambdaregelung, die eingespritzte Kraftstoffmenge, die Motordrehzahl beeinflussen und da umgekehrt die Stelleingriffe der Motordrehzahlregelung, die Drosselklappenstellung, den Lambdawert beeinflussen, legt Roduner [84] ein Mehrgrößenregler aus.

Es ist nicht gewährleistet, dass sich die Abgase der einzelnen Zylinder bis zum Katalysator ideal durchmischen und es kann vorkommen, dass Abgase mit unterschiedlichem Lambdawert den Katalysator blockweise passieren. Dies führt zu einer ineffizienten Konvertierung der Schadstoffe und sollte vermieden werden. Synchronisation der Lambdawerte der einzelnen Zylinder kann Abhilfe schaffen. Dies ist allerdings mit sehr hohen Anforderungen an die Dynamik der Lambdasonde verbunden [81].

\subsection{Modellierung der Motorkomponenten im Lambdaregelkreis}

Dieser Abschnitt ist, wenn keine anderen Quellenangaben gemacht werden, an die Ausführungen in [40] angelehnt. Um weiterführende Recherchen zu erleichtern, wird die Nomenklatur aus [40] weitgehend übernommen.

Die Verbrennung des Luft-Kraftstoff-Gemisches im Motor ist ein hochdynamischer Vorgang, dessen Modellierung mit einem entsprechend hohen Aufwand verbunden ist. Die exakte Modellierung der Temperatur- und der Druckverläufe in den Brennräumen des Motors ist jedoch für die Lambdaregelung nicht notwendig. Unter der Annahme, dass der Lambdawert eine Erhaltungsgröße ist, sollte ein Modell für den Reglerentwurf die in die Brennräume gelangenden Luft- und Kraftstoffmengen wiedergeben können. Die diskrete Arbeitsweise von Hubkolbenmotoren motiviert zu einer ereignisbasierten Modellierung der Vorgänge in den einzelnen Arbeitstakten. Bei Motoren mit mehreren Zylindern sind die Arbeitstakte der einzelnen Zylinder jedoch so zueinander versetzt, dass die Arbeitstakte aller Zylinder bei einem Viertaktmotor möglichst gleichmäßig über zwei Kurbelwellenumdrehungen verteilt sind. Stellt man sich einen Motor mit unendlich vielen Zylindern vor, so würde er kontinuierlich Luft-Kraftstoff-Gemisch ansaugen, verdichten, verbrennen und Abgas ausstoßen. Modelle, die auch bei einer endlichen Anzahl von Zylindern 


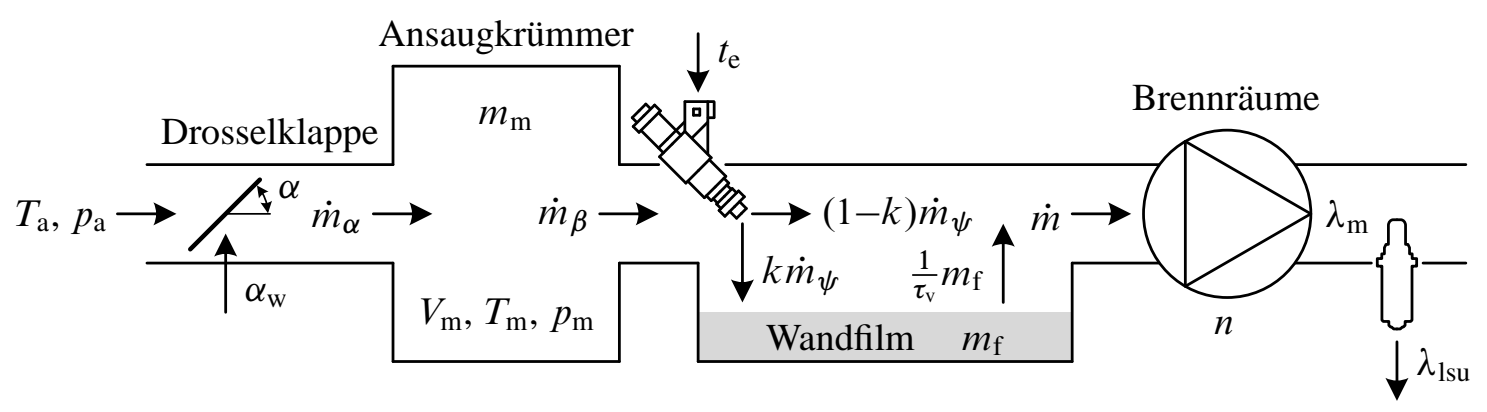

Bild 2.1: Prinzipielle Struktur eines Motors mit Saugrohreinspritzung, in Anlehnung an [40]

die Arbeitstakte von Hubkolbenmotoren gleichmäßig verteilt über den Kreisprozess betrachten, werden Mittelwertmodelle genannt. Mittelwertmodelle sind üblicherweise Modelle mit konzentrierten Parametern und durch das Einführen von Verzögerungen wird die diskrete Natur des Hubkolbenmotors berücksichtigt. Mittelwertmodelle eignen sich gut für die Beschreibung der langsameren Vorgänge im Motor und damit auch für den Entwurf des Lambdaregelkreises [5, 77].

Brennräume In Bild 2.1 ist die prinzipielle Struktur eines Motors mit Saugrohreinspritzung abgebildet. Die Modelle des Luft- und des Kraftstoffpfads beschreiben die Massenströme von Luft bzw. Kraftstoff in den Brennräumen des Motors und berücksichtigen die jeweils wichtigen mechanischen und thermodynamischen Vorgänge. Der Massenstrom $\dot{m}$ durch die Brennräume setzt sich aus dem Luftmassenstrom $\dot{m}_{\beta}$ und dem Kraftstoffmassenstrom $\dot{m}_{\varphi}$ zusammen:

$$
\dot{m}=\dot{m}_{\beta}+\dot{m}_{\varphi} .
$$

Der Motor selbst wird durch eine volumetrische Pumpe dargestellt, durch die der Massenstrom näherungsweise proportional zur Motordrehzahl $n$ ist. Die volumetrische Effizienz $e_{\mathrm{v}}$ in der Gleichung für den Massenstrom $\dot{m}$

$$
\dot{m}=\rho_{\mathrm{in}} e_{\mathrm{v}}\left(p_{\mathrm{m}}, n\right) \frac{V_{\mathrm{d}}}{2} n
$$

beschreibt die Abweichung des realen Motors von einer idealen volumetrischen Pumpe und hängt vom Druck $p_{\mathrm{m}}$ im Ansaugkrümmer und der Motordrehzahl $n$ ab. In Gleichung (2.2) bezeichnet $V_{\mathrm{d}}$ den Motorhubraum und $\rho_{\text {in }}$ die Dichte des Luft-Kraftstoff-Gemisches am Eingang der Brennräume.

Ansaugkrümmer Der Ansaugkrümmer stellt einen Energiespeicher dar, in dem die Energie unter Vernachlässigung der potentiellen und der kinetischen Energie des Massenstroms in Form der inneren Energie der Luft gespeichert ist. Die Änderung der gespeicherten Luftmasse $m_{\mathrm{m}}$ ist durch die Differenz der ein- und der ausströmenden Luftmassenströme gegeben:

$$
\frac{\mathrm{d}}{\mathrm{d} t} m_{\mathrm{m}}=\dot{m}_{\alpha}-\dot{m}_{\beta}
$$

Hierbei bezeichnet $\dot{m}_{\alpha}$ den Luftmassenstrom durch die Drosselklappe, die am Eingang des Ansaugkrümmers angebracht ist. Vernachlässigt man die Temperaturänderung $\Delta T_{\mathrm{m}}$ der Luft im An- 


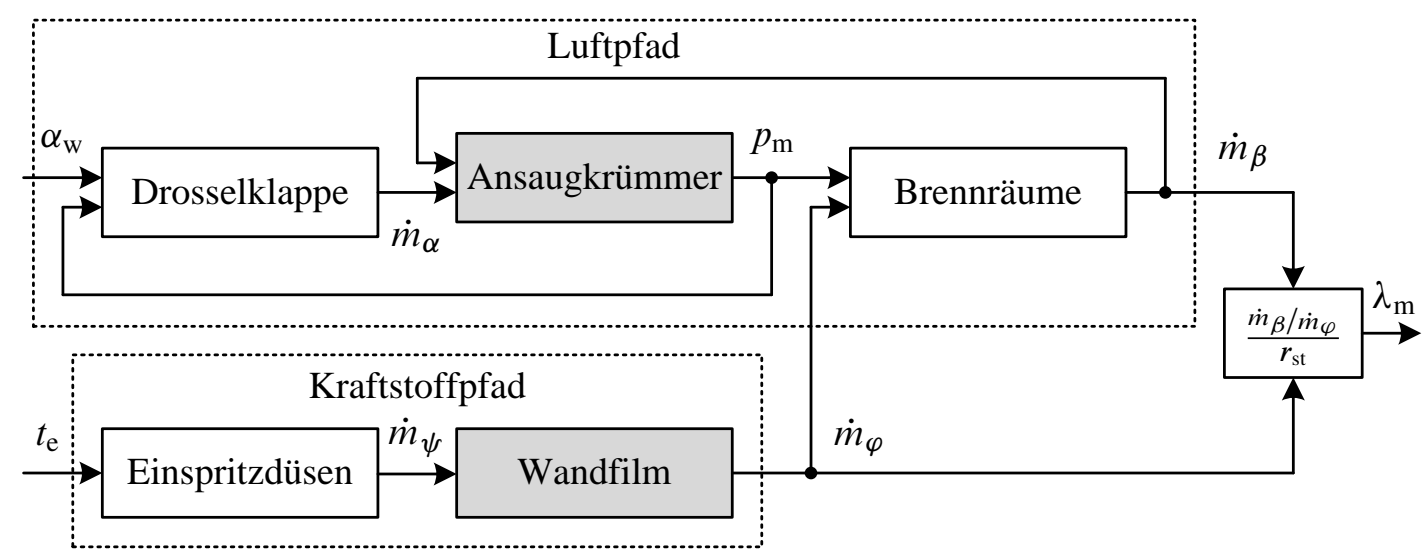

Bild 2.2: Subsysteme in dem Luft- und dem Kraftstoffpfad eines Motors mit Saugrohreinspritzung, in Anlehnung an [40]. Die weißen Blöcke beschreiben funktionale Zusammenhänge und die grauen Blöcke dynamische Vorgänge.

saugkrümmer, so kann durch die thermische Zustandsgleichung für ideale Gase

$$
p V=m \mathrm{R} T
$$

Gleichung (2.3) auf den Druck $p_{\mathrm{m}}$ im Ansaugkrümmer umgestellt werden:

$$
\frac{\mathrm{d}}{\mathrm{d} t} p_{\mathrm{m}}=\frac{\mathrm{R} T_{\mathrm{m}}}{V_{\mathrm{m}}}\left(\dot{m}_{\alpha}-\dot{m}_{\beta}\right) \text {. }
$$

$V_{\mathrm{m}}$ und $T_{\mathrm{m}}$ bezeichnen das Volumen des Ansaugkrümmers und die Temperatur der Luft im Ansaugkrümmer.

Drosselklappe Die Drosselklappe stellt einen Widerstand für die in den Ansaugkrümmer einströmende Luft dar und bewirkt einen Druckabfall vom atmosphärischen Druck $p_{\mathrm{a}}$ auf den Druck im Ansaugkrümmer $p_{\mathrm{m}}$. Der Massenstrom $\dot{m}_{\alpha}$ durch die Drosselklappe kann durch die folgende Gleichung beschrieben werden:

$$
\dot{m}_{\alpha}=\frac{c_{\mathrm{d}} p_{\mathrm{a}} A(\alpha)}{\sqrt{\mathrm{R} T_{\mathrm{a}}}} \Psi\left(\frac{p_{\mathrm{a}}}{p_{\mathrm{m}}}\right) .
$$

Hierbei steht $c_{\mathrm{d}}$ für den Druckverlustbeiwert, $A(\alpha)$ für die vom Öffnungswinkel $\alpha$ abhängige offene Fläche der Drosselklappe, $T_{\mathrm{a}}$ für die Temperatur der einströmende Luft und $\Psi(\cdot)$ für die nichtlineare Flussfunktion, die den Luftmassenstrom begrenzt.

Gesamtmodell des Luftpfads In Bild 2.2 sind die Abhängigkeiten zwischen den einzelnen Subsystemen im Luftpfad dargestellt. Bis auf die Dynamik im Ansaugkrümmer werden die Subsysteme durch nichtlineare funktionale Zusammenhänge beschrieben. In Bild 2.2 ist weiterhin der Kraftstoffpfad bestehend aus der Kraftstoffeinspritzung und der Dynamik der Wandfilmbildung abgebildet. Der durch die Einspritzdüsen fließende Kraftstoffmassenstrom $\dot{m}_{\psi}$ ist näherungsweise proportional zu der Kraftstoffeinspritzzeit $t_{\mathrm{e}}$. Das durch das stöchiometrische Luft-KraftstoffVerhältnis $r_{\text {st }}$ normierte Verhältnis der Massenströme $\dot{m}_{\beta}$ und $\dot{m}_{\varphi}$ ergibt den Lambdawert $\lambda_{\mathrm{m}} \mathrm{im}$ Motorabgas. 
Wandfilmbildung Die Wandfilmbildung beschreibt den Effekt, dass ein Teil des eingespritzten Kraftstoffs nicht sofort die Brennräume erreicht und sich in Form eines Kraftstofffilms an der Saugrohrwand sammelt. Mit der Zeit verdunstet und verdampft der Kraftstoff aus dem Wandfilm und gelangt in die Brennräume des Motors [25, 40]. Zur Beschreibung dieses Effekts haben sich lineare Modelle bewährt, die die Kraftstoffmasse $m_{\mathrm{f}}$ im Wandfilm als dynamische Größe modellieren. Auf die Herleitung der einzelnen Modellgleichungen wird an dieser Stelle verzichtet und auf die entsprechende Literatur, z. B. [40, 66], verwiesen. Nach Einsetzen der Modellgleichungen ineinander erhält man die Gleichung für den in die Brennräume gelangenden Kraftstoffmassenstrom $\dot{m}_{\varphi}$ :

$$
\dot{m}_{\varphi}=(1-k) \dot{m}_{\psi}+\frac{k}{\tau_{\mathrm{v}} s+1} \dot{m}_{\psi} .
$$

Die Konstante $k$ beschreibt den Kraftstoffanteil, der zum Wandfilm beiträgt, während die Zeitkonstante $\tau_{\mathrm{v}}$ die Geschwindigkeit der Verdunstung des Kraftstoffs aus dem Wandfilm angibt. Die Modellparameter $k$ und $\tau_{\mathrm{v}}$ hängen vom Motorarbeitspunkt ab.

Transportzeiten Am Anfang des Abschnitts wird erwähnt, dass durch das Einführen von Verzögerungen der diskreten Natur des Motors Rechnung getragen wird. Durch reine Totzeiten wird der Transport der Luft, des Kraftstoffs und der Abgase modelliert. Bei einem Viertaktmotor werden vom Anfang des Ansaugtakts bis zum Anfang des Ausstoßtakts eineinhalb Kurbelwellenumdrehungen benötigt. Unter Vernachlässigung der Verzögerungen bei Betätigung der Einspritzventile und Asymmetrien beim Öffnen und Schließen der Ein- und der Auslassventile gilt damit für die Transportzeit des Lambdawertes durch den Motor:

$$
\tau_{\mathrm{d}} \approx \frac{3}{2 n} \text {. }
$$

Eine weitere Totzeit $\tau_{\mathrm{ab}}$ resultiert aus dem Transport der Abgase vom Abgaskrümmer bis zur Lambdasonde, welche typischerweise im Bereich von 20 bis $500 \mathrm{~ms}$ je nach Abgasmassenstrom liegt.

Die Abgase der einzelnen Zylinder erreichen den Abgaskrümmer jeweils um den zeitlichen Zündabstand nacheinander versetzt und so resultiert aus einem Sollwertsprung in Lambda eine treppenförmige Änderung des Lambdawertes im Abgaskrümmer, siehe Bild 2.3. Diese treppenförmige Änderung des Lambdawertes im Motorabgas wird oft durch den Verlauf eines PT ${ }_{1}$-Gliedes mit der Zeitkonstante $\tau_{\mathrm{m}}$ approximiert [40, 49]. In [49] wird diese Zeitkonstante so gewählt, dass sie dem $\left(n_{\text {cyl }}-1\right)$-fachen Zündabstand entspricht:

$$
\tau_{\mathrm{m}}=\frac{2}{n_{\mathrm{cyl}} n}\left(n_{\mathrm{cyl}}-1\right) .
$$

In Gleichung (2.9) bezeichnet $n_{\text {cyl }}$ die Zylinderanzahl des Motors.

Lambdasonde Ebenfalls als ein $\mathrm{PT}_{1}$-Glied mit einer Zeitkonstante $\tau_{\text {lsu }}$ im Bereich von $50 \mathrm{~ms}$ bis $1 \mathrm{~s}$ wird die Dynamik der Lambdasonde modelliert [30, 40, 77]. Dabei gilt der kleinere Wert für neuwertige Breitband-Lambdasonden und infolge der Alterung kann die Zeitkonstante bis zum größeren Wert ansteigen [86]. 


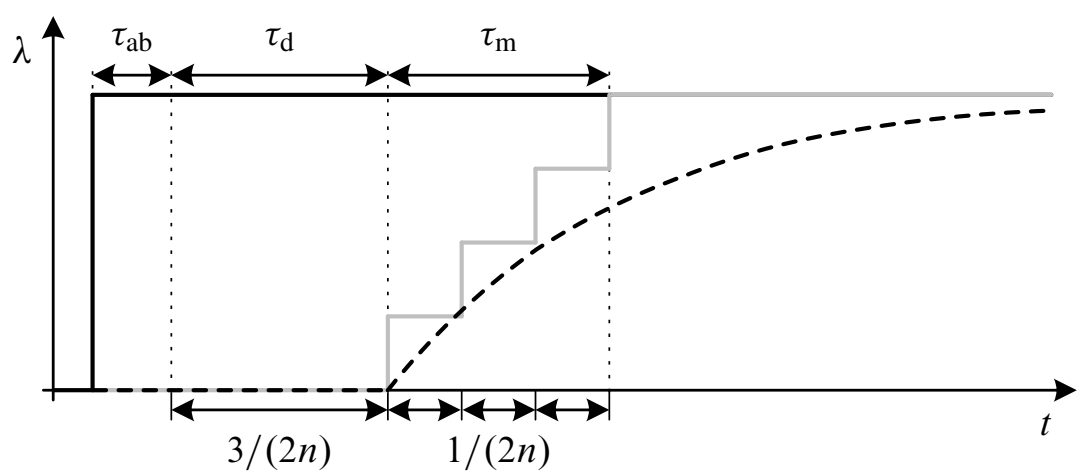

Bild 2.3: Approximation der Gasmischung im Abgaskrümmer durch den Verlauf eines $\mathrm{PT}_{1}$ Gliedes, aus [49]: Soll-Lambdawert $(-)$, vereinfachte Sprungantwort $(-)$ und $\mathrm{PT}_{1}$ Approximation (----)

\subsection{Regelung des Lambdawertes}

Die Stellgröße im Lambdaregelkreis ist die Kraftstoffeinspritzzeit $t_{\mathrm{e}}$. Über ihre Verkürzung oder Verlängerung kann das Luft-Kraftstoff-Verhältnis im Ansaugkrümmer beeinflusst werden. Die einzuspritzende Kraftstoffmenge wird aus dem Soll-Lambdawert $\lambda_{\mathrm{w}}$ und der in die Brennräume gelangenden Luftmenge bestimmt. Die Verstellung der Drosselklappe durch den Fahrer oder durch eine Drehzahlregelung stellt eine Störgröße für den Lambdaregelkreis dar. Aufgrund der Verzögerungen und Totzeiten zwischen der Kraftstoffeinspritzung und der Erfassung des Lambdawertes im Motorabgas ist eine klassische einschleifige Regelung zu langsam. Daher wird in der Regel eine Zwei-Freiheitsgrade-Regelkreisstruktur mit einer Vorsteuerung der Kraftstoffeinspritzung und einem Lambdaregler eingesetzt [5, 40, 47]. Die Vorsteuerung basiert auf Beobachtern für die in die Brennräume gelangende Luftmenge und einer Kompensation des Einflusses des Luftpfades auf den Lambdawert. Um die Schätzung des Luftmassenstroms zu verbessern, basieren die Beobachter vorzugsweise nicht auf Mittelwertmodellen, sondern auf ereignisbasierten Modellen des Luftpfades. Sie werden als Open-Loop-Beobachter implementiert und mit den Messsignalen des Luftmassenmessers, des Drehzahlmessers, des Winkelsensors der Drosselklappe usw. gespeist [40].

Der Effekt der Wandfilmbildung im Kraftstoffpfad kann in der Vorsteuerung durch die Inversion des Modells in Gleichung (2.7) kompensiert werden. Bis auf die Totzeiten kann der Kraftstoffpfad somit als eine Verstärkung modelliert werden. Der Lambdaregler sorgt für die stationäre Genauigkeit des Lambdaregelkreises, die aufgrund des Open-Loop-Beobachters in der Vorsteuerung nicht gewährleistet ist. Nach der Kompensation des Effekts der Wandfilmbildung besteht das Motormodell $\tilde{P}$ für den Reglerentwurf aus einem PT $_{1}$-Glied, das die Gasmischung im Abgaskrümmer approximiert, und einer Totzeit, die die Transportzeiten widerspiegelt [77, 78, 85, 86]:

$$
\lambda_{\mathrm{m}}=\underbrace{\frac{1}{\tau_{\mathrm{m}}(M, n) s+1} \mathrm{e}^{-\tau_{\mathrm{d}}(M, n) s}}_{\tilde{P}} \lambda_{\mathrm{u}} .
$$




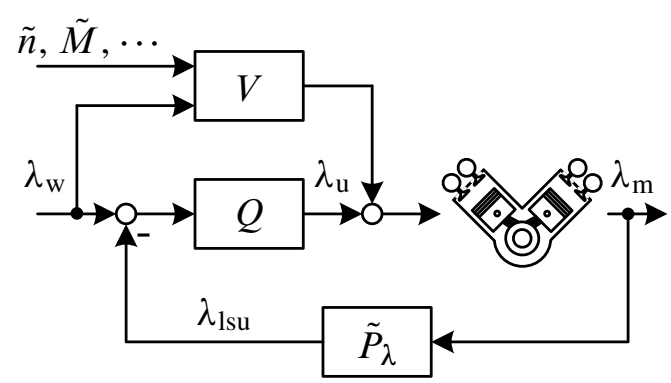

Bild 2.4: Strukturbild des Lambdaregelkreises mit nichtlinearer Vorsteuerung $V$ der Kraftstoffeinspritzung und linearem Lambdaregler $Q$

Die Werte der Zeitkonstante $\tau_{\mathrm{m}}$ und der Totzeit $\tau_{\mathrm{d}}$ hängen von der Motordrehzahl $n$ und dem Motorlastmoment $M$ ab. Sie können alternativ zu den Abschätzungen in den Gleichungen (2.9) und (2.8) messtechnisch ermittelt und in Kennfeldern abgespeichert werden. Wenngleich die reale Stellgröße die Kraftstoffeinspritzzeit $t_{\mathrm{e}}$ ist, wird in dieser Arbeit als Stellgröße der Lambdawert $\lambda_{\mathrm{u}}$ verwendet. Bei Kenntnis des geschätzten Luftmassenstroms kann der Lambdawert $\lambda_{\mathrm{u}}$ in die Kraftstoffeinspritzzeit $t_{\mathrm{e}}$ umgerechnet werden. In Bild 2.4 ist das Strukturbild des Lambdaregelkreises abgebildet. Der Soll-Lambdawert ist durch $\lambda_{\mathrm{w}}$, die Vorsteuerung durch $V$ und die linearen Übertragungsfunktionen des Lambdaregelers und der Lambdasonde sind durch $Q$ bzw. $\tilde{P}_{\lambda}$ gekennzeichnet. Die symbolische Darstellung des Motors in Bild 2.4 soll auf sein nichtlineares Verhalten hindeuten.

\subsubsection{Anforderungen an den Lambdaregelkreis und Randbedingungen}

Die schnelle aber approximative Vorsteuerung der Kraftstoffeinspritzung führt zu einer unerwünschten Lambdaabweichung vom Sollwert im Motorabgas, die vom Lambdaregler ausgeregelt werden soll [40]. Die Modellierung dieser Lambdaabweichung ist kompliziert und erfordert ein detailliertes Modell des Antriebsstrangs des Fahrzeugs und die Simulation von Fahrzyklen. Die Kenntnis des Amplitudenspektrums der Lambdaabweichung ist jedoch wichtig, da sie die gewünschte Bandbreite des Lambdaregelkreises bestimmt und bei der Auslegung des Katalysators nützlich sein kann. Möller [64] macht den Ansatz, den Verlauf der Lambdaabweichung experimentell zu ermitteln und in der Simulationsumgebung zu berücksichtigen. Dafür wird das letzte Drittel des Federal Test Procedure 75 (FTP 75-Fahrzyklus) an einem Motorprüfstand nachgefahren, der Lambdawert im Motorabgas aufgenommen und dabei die Kraftstoffeinspritzung alleine durch die Vorsteuerung vorgenommen. Aus den Messdaten wird anschließend die Lambdaabweichung ermittelt und in der Simulationsumgebung zum Ausgang des Motormodells addiert. Bei dieser Vorgehensweise wird zwar der Einfluss des Lambdareglers selbst auf die Lambdaabweichung vernachlässigt, Möller gibt jedoch eine gute Übereinstimmung zwischen Simulationen und Messungen am Motorprüfstand mit aktivierter Lambdaregelung an.

Wie bereits erläutert, kompensiert die Vorsteuerung $V$ das nichtlineare Motorverhalten, sodass das lineare Motormodell in Gleichung (2.10) für den Reglerentwurf eingesetzt werden kann. Folglich vereinfacht sich das Strukturbild in Bild 2.4 zu jenem in Bild 2.5. Die Lambdaabweichung, die 


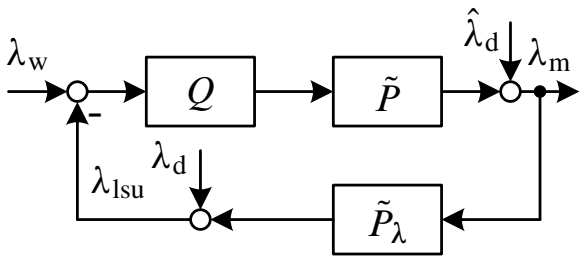

Bild 2.5: Strukturbild des Lambdaregelkreises nach Kompensation des nichtlinearen Motorverhaltens

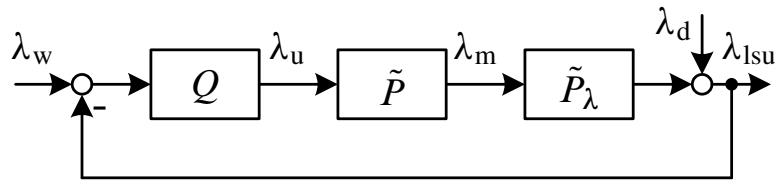

Bild 2.6: Strukturbild des Lambdaregelkreises für den Reglerentwurf

von der Vorsteuerung verursacht wird, wird durch die additive Störung $\hat{\lambda}_{\mathrm{d}}$ hinter dem Motormodell $\tilde{P}$ repräsentiert. Bei der experimentellen Ermittlung der Lambdaabweichung wird jedoch die Abweichung des Messsignals $\lambda_{\text {lsu }}$ der Lambdasonde vom stöchiometrischen Wert gemessen und damit die Summe aus den Messfehlern $\lambda_{\mathrm{d}}$ der Lambdasonde selbst und der durch die Dynamik der Lambdasonde gefilterten Lambdaabweichung $\hat{\lambda}_{\mathrm{d}}$, siehe Bild 2.5. Das von Möller aufgenommene Signal $\lambda_{\text {lsu }}$ steht messtechnisch zur Verfügung, die Dynamik der Lambdasonde in Abhängigkeit des Motorarbeitspunktes und ihre Messfehler sind jedoch unbekannt. Daher ist die Berechnung der Lambdaabweichung $\hat{\lambda}_{d}$ nicht möglich. Aus diesem Grund wird in dieser Arbeit die vereinfachte Struktur in Bild 2.6 mit der Reihenschaltung der Modelle von Motor und Lambdasonde als zu regelnde Strecke betrachtet. Dabei werden alle Störungen zu der gemessenen Lambdaabweichung $\lambda_{\mathrm{d}}$ zusammengefasst.

Regelziel In Bild 2.7 ist der Zeitverlauf der von Möller an einem Motorprüfstand aufgenommenen Lambdaabweichung $\lambda_{\mathrm{d}}$ dargestellt. Diese bewegt sich im Intervall von $-23 \%$ bis $14 \%$ bezogen auf den stöchiometrischen Lambdawert. Die Amplitude von $\lambda_{\mathrm{d}}$ ist $95 \%$ der Zeit kleiner als $5 \%$ und etwa $50 \%$ der Zeit sogar kleiner als 1\%. Neben der Lambdaabweichung sind in Bild 2.7 noch die Verläufe der Motordrehzahl $n$ und des relativen Motorlastmoments $M_{\text {rel }}$ dargestellt. Die größeren Amplituden von $\lambda_{d}$ korrelieren mit starken Änderungen der Motordrehzahl und des Motorlastmoments. Das dazugehörige Amplitudenspektrum der Lambdaabweichung ist in Bild 2.8 dargestellt. Die größten Amplituden liegen im Intervall von 0 bis etwa $0,5 \mathrm{~Hz}$ und ab etwa $1 \mathrm{~Hz}$ nehmen die Amplituden nur noch langsam ab. Daraus lässt sich auch das Regelziel beim Entwurf des Lambdareglers ableiten: Es wird eine Bandbreite von etwa 0,5 Hz bis $1 \mathrm{~Hz}$ unter Einhaltung der Stellgrößenbeschränkung angestrebt.

An dieser Stelle wird nicht berücksichtigt, dass die Lambdaabweichung in Bild 2.7 bei wechselnder Motordrehzahl und wechselndem Motorlastmoment aufgenommen worden ist und damit für jeden Motorarbeitspunkt ein anderer Verlauf der Störung relevant ist. Es wird hier jedoch vereinfachend angenommen, dass die gewünschte Bandbreite für alle Motorarbeitspunkte gleich ist.

Stellgrößenbeschränkung Der Lambdabereich, in dem das Luft-Kraftstoff-Gemisch in einem Motor mit Saugrohreinspritzung gezündet und verbrannt werden kann, erstreckt sich von etwa 0,5 bis 1,7. Dabei wird die höchste Motorleistung bei einem Lambdawert von etwa 0,9 und die höchste Kraftstoffeffizienz bei einem Lambdawert zwischen 1,1 und 1,3 erreicht [25, 36]. Für die 
Relative Motorlast

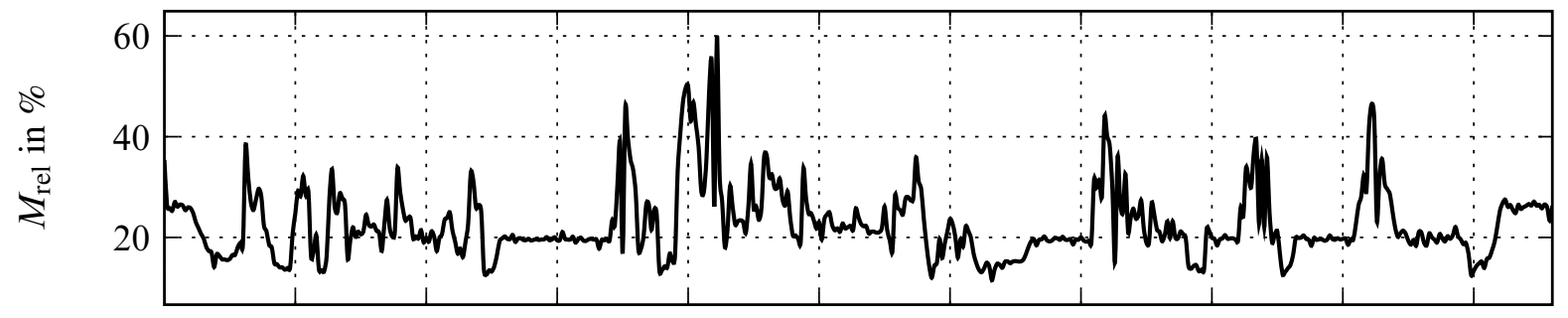

Motordrehzahl

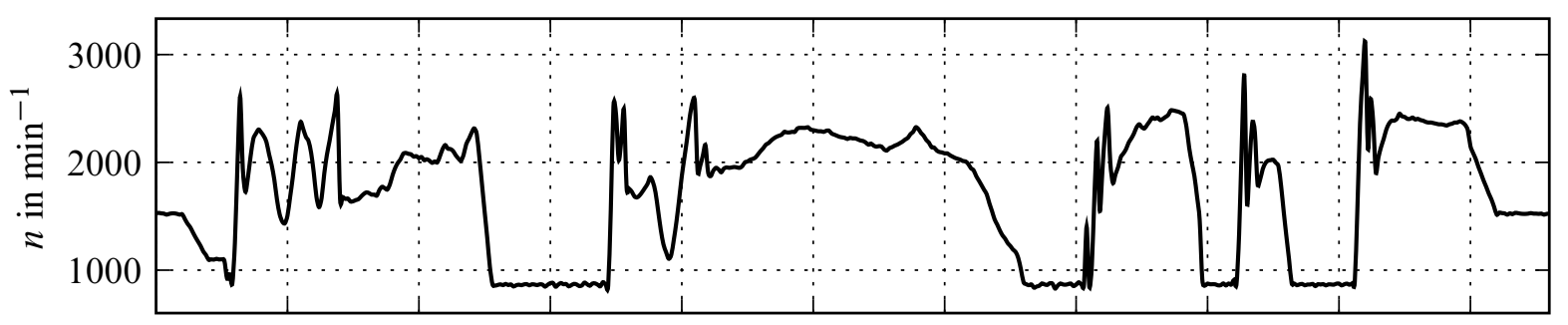

Lambdaabweichung

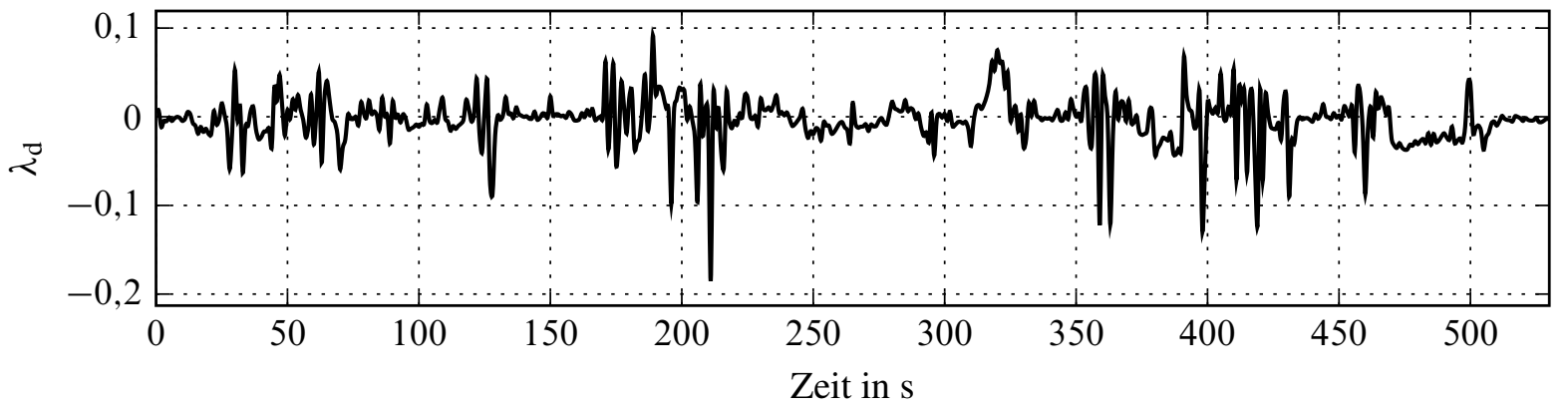

Bild 2.7: Zeitverlauf der von Möller [64] gemessenen Lambdaabweichung $\lambda_{\mathrm{d}}$ beim Nachfahren der letzten $540 \mathrm{~s}$ des FTP 75-Fahrzyklus an einem Motorprüfstand

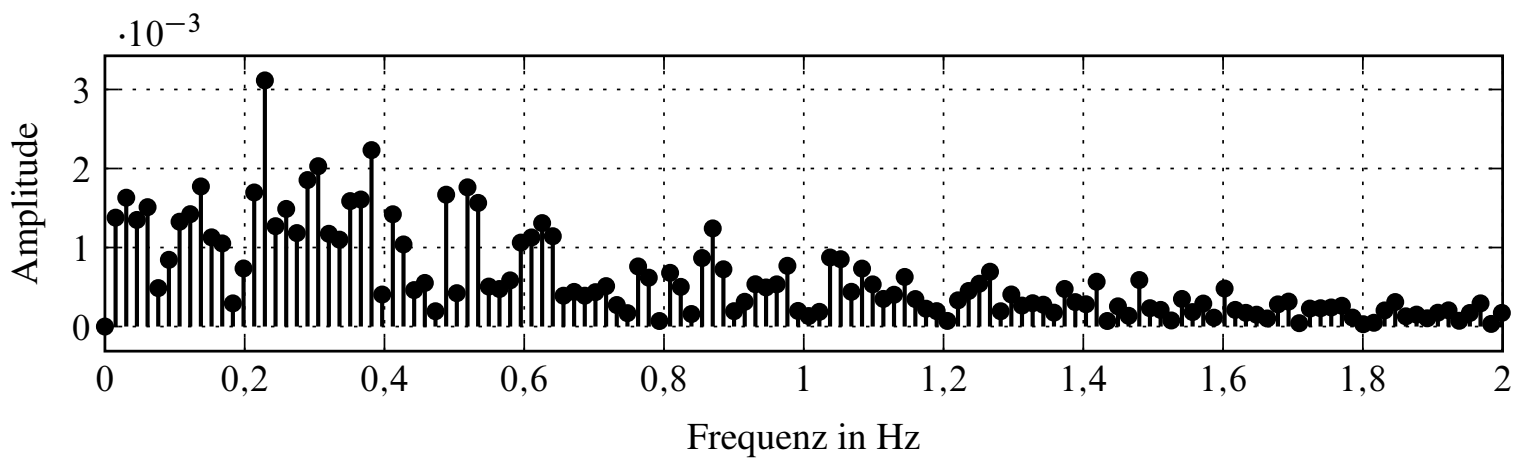

Bild 2.8: Amplitudenspektrum der gemessenen Lambdaabweichung $\lambda_{\mathrm{d}}$ in Bild 2.7

Lambdaregelung steht jedoch ein kleinerer Lambdabereich von etwa 0,9 bis 1,1 zur Verfügung, da sich kleinere oder größere Lambdawerte negativ auf die Fahrbarkeit des Fahrzeugs auswirken können. 


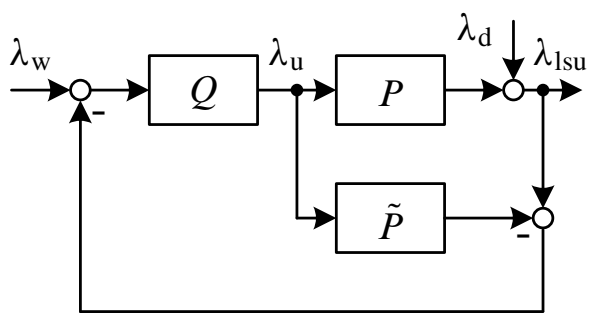

Bild 2.9: Strukturbild eines Regelkreises in der IMC-Struktur

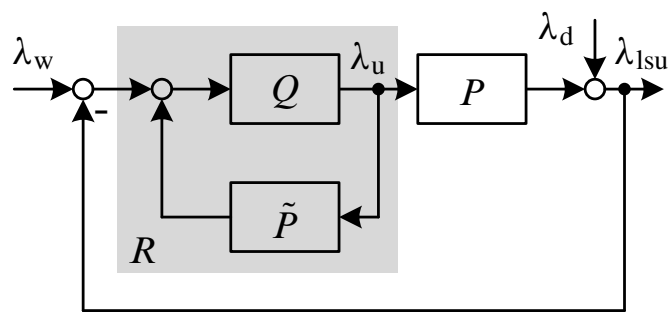

Bild 2.10: Umformung der IMC-Struktur in die klassische Regelkreisstruktur

\subsubsection{Die IMC-Struktur}

Für den Lambdaregelkreis bietet es sich an, eine Regelkreisstruktur zu wählen, die die systematische Berücksichtigung der Totzeit im Motormodell (2.10) und die Stellgrößenbeschränkung auf einfache Weise ermöglicht. Für stabile Systeme kann hierfür die IMC-Struktur eingesetzt werden, die von Morari und Zafiriou [67] ausführlich beschrieben wird. An dieser Quelle orientieren sich auch die Ausführungen und die Nomenklatur in diesem Abschnitt.

In Bild 2.9 ist das Strukturbild eines Regelkreises in der IMC-Struktur abgebildet. Dabei bezeichnet $Q$ den IMC-Regler, $P$ die zu regelnde Strecke und $\tilde{P}$ das Modell der Strecke. Die IMCStruktur kann jederzeit in die klassische Regelkreisstruktur nach der Vorschrift

$$
R=\frac{Q}{1-\tilde{P} Q}
$$

umgeformt werden, siehe Bild 2.10. Vom Vorhandensein des Modells $\tilde{P}$ der Strecke in der Reglerstruktur leitet sich die Bezeichnung Internal Model Control ab. Nimmt man an, dass das Modell $\tilde{P}$ keinen Modellunsicherheiten unterliegt und keine Störung $\lambda_{\mathrm{d}}$ vorhanden ist, so lautet die Führungsübertragungsfunktion des IMC-Regelkreises:

$$
G_{\mathrm{w}}=Q P .
$$

Gleichung (2.12) impliziert, dass für eine stabile Strecke $P$ jeder stabile IMC-Regler $Q$ zu einem stabilen geschlossenen Regelkreis führt. Im klassischen Regelkreis lautet die Führungsübertragungsfunktion

$$
G_{\mathrm{w}}=\frac{R P}{1+R P}
$$

und sie ist deutlich komplexer als Gleichung (2.12). Die Suche nach einem IMC-Regler, der die Stabilitäts- und die Performanceanforderungen erfüllt, wird dadurch erleichtert, dass der IMCRegler $Q$ linear in die Führungsübertragungsfunktion $G_{\mathrm{w}}$ und in die Störübertragungsfunktion $G_{\mathrm{d}}$

$$
G_{\mathrm{d}}=1-P Q
$$

eingeht. Das Ziel jedes Reglerentwurfs ist die Gestaltung dieser zwei Funktionen im Hinblick auf die Performance $\left(G_{\mathrm{d}}\right)$ und die Robustheit $\left(G_{\mathrm{w}}\right)$ des geschlossenen Regelkreises. 


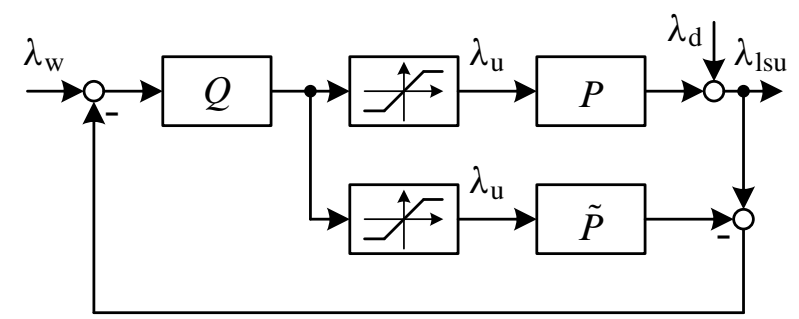

Bild 2.11: Begrenzen der Stellgröße in der IMC-Struktur

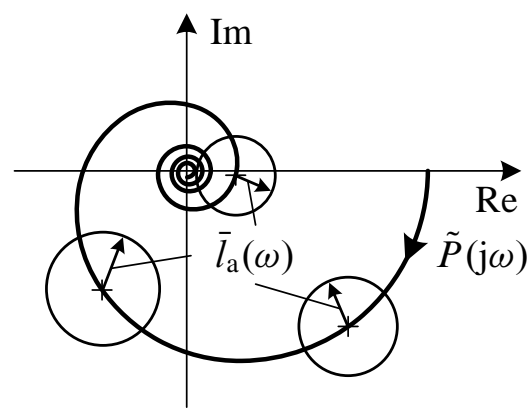

Bild 2.12: Additive Modellunsicherheit in der komplexen s-Ebene, aus [97]

Aber nicht nur beim Reglerentwurf bietet die IMC-Struktur Vorteile gegenüber der klassischen Regelkreisstruktur. Eine Stellgrößenbeschränkung kann in der klassischen Regelkreisstruktur zu einer Instabilität des Regelkreises führen und den Einsatz von zusätzlichen Anti-Windup-Strategien notwendig machen. Im klassischen Regelkreis wird die Stellgröße der Strecke $P$, jedoch nicht des Modells $\tilde{P}$ in Bild 2.10 beschränkt. Dadurch verhalten sich Strecke und Modell unterschiedlich und es kann zu einem instabilen Verhalten kommen. Im Fall der IMC-Struktur wird eine vorhandene Stellgrößenbeschränkung dagegen so implementiert, dass die Stellgröße sowohl der Strecke $P$ als auch des Modells $\tilde{P}$ beschränkt wird, siehe Bild 2.11. Unter der Annahme, dass keine Störung und keine Modellunsicherheiten vorhanden sind, bestimmt sich die Stabilität des Regelkreises in diesem Fall weiterhin durch die Stabilität des IMC-Reglers und der Strecke selbst. Da jedoch für $\tilde{P}=P$ keine Rückkopplung existiert, bleibt der IMC-Regler $Q$ im Ungewissen über die Stellgrößenbeschränkung und dies kann die Performance des Regelkreises im Vergleich zu einer Anti-Windup-Strategie reduzieren.

Modellunsicherheiten Da das Modell $\tilde{P}$ im Allgemeinen nicht exakt die reale Strecke $P$ beschreibt, wird für den Reglerentwurf noch eine Beschreibung der Modellunsicherheit benötigt. Alle Ursachen für die Modellabweichung wie Parameter- und Strukturunsicherheiten werden zusammengefasst und damit eine unstrukturierte Beschreibung der Modellunsicherheit vorgenommen. Im Frequenzbereich können Modellunsicherheiten durch kreisförmige Regionen mit dem Radius $\bar{l}_{\mathrm{a}}(\omega)$ um jeden Punkt der Ortskurve des nominellen Modells $\tilde{P}(\mathrm{j} \omega)$ beschrieben werden, siehe Bild 2.12. $l_{\mathrm{a}}(\mathrm{j} \omega)$ ist die additive Modellunsicherheit und für die Modelle $P(\mathrm{j} \omega)$ aus der betrachteten Modellfamilie $\Pi$ gilt

$$
P(\mathrm{j} \omega)=\tilde{P}(\mathrm{j} \omega)+l_{\mathrm{a}}(\mathrm{j} \omega)
$$

mit der oberen Schranke $\bar{l}_{\mathrm{a}}(\omega)$ :

$$
\left|l_{\mathrm{a}}(\mathrm{j} \omega)\right| \leq \bar{l}_{\mathrm{a}}(\omega)
$$

Die additive Modellunsicherheit kann auch in eine multiplikative Modellunsicherheit $l_{\mathrm{m}}(\mathrm{j} \omega) \mathrm{um}$ gerechnet werden, sodass sich die Modelle $P(\mathrm{j} \omega)$ aus der betrachteten Modellfamilie $\Pi$ durch

$$
P(\mathrm{j} \omega)=\tilde{P}(\mathrm{j} \omega)\left(1+l_{\mathrm{m}}(\mathrm{j} \omega)\right)
$$


mit der oberen Schranke $\bar{l}_{\mathrm{m}}(\omega)$ darstellen lassen [67, 97]:

$$
\left|l_{\mathrm{m}}(\mathrm{j} \omega)\right| \leq \bar{l}_{\mathrm{m}}(\omega)
$$

IMC-Reglerentwurf Morari und Zafiriou führen den Reglerentwurf in zwei Schritten durch: Im ersten Schritt wird ein $\mathrm{H}_{2}$-optimaler Regler $\tilde{Q}$ für das nominelle Streckenmodell $\tilde{P}$ entworfen und im zweiten Schritt wird der Regler $\tilde{Q}$ um ein Filter $F$ erweitert. Das Filter sorgt für die Kausalität des resultierenden IMC-Reglers $Q$ und für die Erfüllung der Stabilitäts- und der Performanceanforderungen. Die Bedingung für nominelle Performance lautet

$$
\left\|G_{\mathrm{d}} w\right\|_{\infty}<1 \text { für } \forall P \in \Pi
$$

mit dem komplexen Gewicht $w$, dessen invertierter Betrag eine obere Schranke für den Betrag der Störübertragungsfunktion $G_{\mathrm{d}}$ darstellt. Die Performanceanforderungen, d. h. gutes Führungsverhalten und die Fähigkeit, Störungen und Messrauschen zu unterdrücken, fließen bei der Wahl des Gewichts $w$ ein. Die Bedingung für robuste Stabilität lautet:

$$
\left\|\tilde{G}_{\mathrm{w}} \bar{l}_{\mathrm{m}}\right\|_{\infty}<1 \text { mit } \tilde{G}_{\mathrm{w}}=\tilde{P} \tilde{Q} F .
$$

Vergleicht man die Ungleichungen (2.20) und (2.19) miteinander, so stellt man fest, dass der invertierte Betrag der maximalen multiplikativen Modellunsicherheit $\bar{l}_{\mathrm{m}}$ eine obere Schranke für die Führungsübertragungsfunktion $\tilde{G}_{\mathrm{w}}$ darstellt. Da der Betrag der Modellunsicherheit typischerweise mit der Frequenz ansteigt, wird der Frequenzbereich mit einem guten Führungsverhalten umso stärker beschränkt, je größer die Modellunsicherheit ist [67, 97]. Die gleichzeitige Berücksichtigung der Performance- (2.19) und der Stabilitätsanforderung (2.20) führt auf die folgende Bedingung für robuste Performance:

$$
\left|\tilde{G}_{\mathrm{d}} w\right|+\left|\tilde{G}_{\mathrm{w}} \bar{l}_{\mathrm{m}}\right|=|(1-\tilde{P} \tilde{Q} F) w|+\left|\tilde{P} \tilde{Q} F \bar{l}_{\mathrm{m}}\right|<1 \text { für } \forall \omega .
$$

Mit dem Tiefpassfilter $F$ wird der erste Term in der Ungleichung (2.21) größer und der zweite Term kleiner gemacht, d. h. es muss ein Kompromiss zwischen Performance und robuste Stabilität gefunden werden. Dieser zweischrittige Reglerentwurf ist nicht optimal bezüglich einer Norm, er ist jedoch sehr transparent und einfach durchzuführen und zur Erfüllung der meisten praktischen Anforderungen ausreichend [67].

\subsubsection{Entwurf des Lambdareglers}

Im Hinblick auf die Implementierung der Lambdaregelung auf einem Motorsteuergerät oder auf dem Laborprüfstand wird der Reglerentwurf diskret durchgeführt. Die Übertragungsfunktion der zu regelnden Strecke lautet im $s$-Bereich entsprechend Bild 2.6

$$
\tilde{P}(s)=\frac{\lambda_{\mathrm{lsu}}(s)}{\lambda_{\mathrm{u}}(s)}=\frac{1}{\tau_{\mathrm{m}}(M, n) s+1} \frac{1}{\tau_{\mathrm{lsu}} s+1} \mathrm{e}^{-\tau_{\mathrm{d}}(M, n) s}
$$


a)

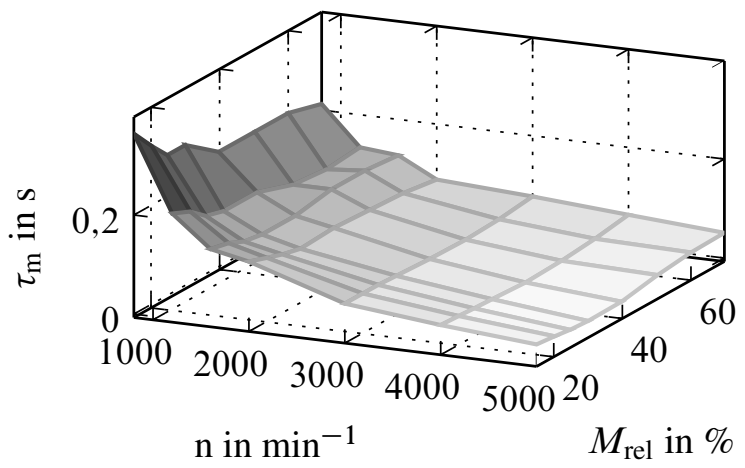

b)

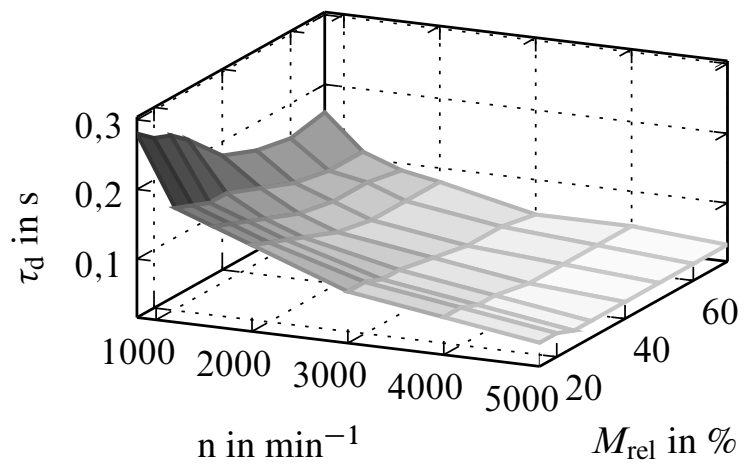

Bild 2.13: a) Zeitkonstante und b) Totzeit des Motormodells in Abhängigkeit der Motordrehzahl und des Motorlastmoments

mit der Totzeit $\tau_{\mathrm{d}}$, die auch die Transportzeit des Motorabgases bis zur Lambdasonde enthält. Die Motorzeitkonstante $\tau_{\mathrm{m}}$ und die Totzeit $\tau_{\mathrm{d}}$ hängen vom Motorarbeitspunkt ab, siehe Bild 2.13, werden aber vorerst nur an einem festen Motorarbeitspunkt betrachtet. Die Daten stammen aus einem Vorgängerprojekt, dessen Ergebnisse von Möller in [64] dargestellt werden. Weiterhin wird die Dynamik der Breitband-Lambdasonde als zeitinvariant und die Übertragungsfunktion des analogen Antialiasing-Filters als ideal angenommen.

Unter Berücksichtigung des Abtast-Halteglieds $H_{0}(s)$ mit der Abtastzeit $\tau$ und des Zusammenhangs $z=e^{\tau s}$ zwischen den komplexen Variablen der $\mathcal{Z}$ - und der Laplace-Transformation gilt für die diskrete Übertragungsfunktion $\tilde{P}^{*}(z)$ :

$$
\tilde{P}^{*}(z)=\mathcal{Z}\left\{H_{0}(s) \tilde{P}(s)\right\}=\frac{k\left(z+n_{1}\right)}{\left(z-\mathrm{e}^{-\tau / \tau_{\mathrm{m}}}\right)\left(z-\mathrm{e}^{-\tau / \tau_{\mathrm{lsu}}}\right)} \frac{1}{z^{N}} .
$$

Hierbei bezeichnet $N$ die Anzahl der Abtastschritte, durch die die Totzeit $\tau_{\mathrm{d}}$ approximiert wird. Die Bezeichnung $(\cdot)^{*}$ der diskreten Übertragungsfunktion soll darauf hinweisen, dass bei der Transformation der kontinuierlichen Übertragungsfunktion in den z-Bereich das Abtast-Halteglied berücksichtigt wird. Die Umrechnung der Polstellen der zeitkontinuierlichen Übertragungsfunktion $\tilde{P}(s)$ in die Polstellen der diskreten Übertragungsfunktion $\tilde{P}^{*}(z)$ ist einfach, während für die Nullstellen keine einfache Vorschrift existiert [67]. Der Übersichtlichkeit halber wird der Zähler der Übertragungsfunktion (2.23) nicht ausgeschrieben und durch die Verstärkung $k$ und die Nullstelle $n_{1}$ dargestellt. Bei der Diskretisierung kann es passieren, dass eine minimalphasige, zeitkontinuierliche Strecke nach der $\mathcal{Z}$-Transformation Nullstellen außerhalb des Einheitskreises hat [67]. Für die praxisrelevanten Bereiche der Modellparameter, siehe Bild 2.13, liegt die Nullstelle der Übertragungsfunktion (2.23) jedoch innerhalb des Einheitskreises.

$\mathbf{H}_{2}$-optimaler Reglerentwurf Im ersten Schritt des IMC-Reglerentwurfs wird der $\mathrm{H}_{2}$-optimale Regler $\tilde{Q}(z)$ entworfen, der für sprungförmige Änderungen der Führungs- und der Störgröße die Inverse des minimalphasigen Teils des Modells $\tilde{P}(z)$ darstellt. Im diskreten Fall ist der minimalphasige Teil so zu definieren, dass er den gleichen Zähler- und Nennergrad besitzt. Damit gilt für 
den $\mathrm{H}_{2}$-optimalen Regler

$$
\tilde{Q}_{\mathrm{H}}(z)=\frac{\left(z-\mathrm{e}^{-\tau / \tau_{\mathrm{m}}}\right)\left(z-\mathrm{e}^{-\tau / \tau_{\mathrm{su}}}\right)}{z k\left(z-n_{1}\right)},
$$

wobei der Index $\mathrm{H}$ auf eine notwendige Modifikation hinweist. Denn Polstellen auf der negativen reellen Achse der komplexen $z$-Ebene entsprechen konjugiert komplexen Polstellen in der komplexen s-Ebene und bewirken damit eine unerwünschte Schwingung der Regelgröße zwischen den Abtastzeitpunkten. Um dies zu verhindern, werden die $\rho$ Polstellen von $\tilde{Q}_{\mathrm{H}}(z)$, die auf der negativen reellen Achse liegen, durch Polstellen im Ursprung ersetzt. Dafür wird der Ausdruck $\tilde{Q}_{-}(z)$ definiert

$$
\tilde{Q}_{-}(z)=\frac{1}{z^{\rho}} \prod_{j=1}^{\rho} \frac{z-n_{j}}{1-n_{j}}
$$

und mit der Übertragungsfunktion $\tilde{Q}_{\mathrm{H}}(z)$ multipliziert:

$$
\tilde{Q}(z)=\tilde{Q}_{\mathrm{H}}(z) \tilde{Q}_{-}(z) .
$$

Bei der Verschiebung der Polstellen darf der Systemtyp, d.h. die Eigenschaft der Regelgröße für vorgegebene Klassen von Führungs- und Störsignalen der Führungsgröße asymptotisch zu folgen, nicht verändert werden [59, 67]. Für die hier gewählten sprungförmigen Änderungen der Führungs- und der Störgröße verändert der Ausdruck (2.25) den Systemtyp 1 nicht und für $\tilde{Q}(z)$ ergibt sich die folgende Übertragungsfunktion:

$$
\tilde{Q}(z)=\frac{\left(z-\mathrm{e}^{-\tau / \tau_{\mathrm{m}}}\right)\left(z-\mathrm{e}^{-\tau / \tau_{\mathrm{su}}}\right)}{k\left(1-n_{1}\right) z^{2}} .
$$

Es kann gezeigt werden, dass der Regler $\tilde{Q}(z)$ die Vorteile eines $\mathrm{H}_{2}$-optimalen Reglerentwurfs und eines Reglerentwurfs auf endliche Einstellzeit kombiniert [67].

Filterentwurf Wie im vorherigen Abschnitt erläutert, wird im nächsten Schritt der auf nominelle Performance ausgelegte Regler $\tilde{Q}(z)$ um ein Tiefpassfilter $F(z)$ erweitert. Nachdem der Regler $\tilde{Q}(z)$ die Dynamik der Strecke bis auf die Nullstelle und die Totzeit kompensiert, bestimmt das Filter die Dynamik des Lambdaregelkreises. Das Filter darf ebenfalls den Systemtyp nicht verändern und kann im vorliegenden Fall zu

$$
F(z)=\frac{(1-\alpha)^{2} z^{2}}{(z-\alpha)^{2}}
$$

gewählt werden. Es ist sinnvoll, ein Filter zweiter Ordnung zu wählen und damit die Ordnung der zu regelnden Strecke beizubehalten. Die Filterauslegung wird so gewählt, dass der Parameter $\alpha$ iterativ erhöht wird, bis die Bedingungen für robuste Stabilität und robuste Performance erfüllt sind.

Robuste Stabilität und robuste Performance Die Bedingung für robuste Stabilität beim diskreten Reglerentwurf lautet

$$
\left|\tilde{P}^{*}\left(\mathrm{e}^{\mathrm{j} \omega \tau}\right) \tilde{Q}\left(\mathrm{e}^{\mathrm{j} \omega \tau}\right) F\left(\mathrm{e}^{\mathrm{j} \omega \tau}\right)\right| \bar{l}_{\mathrm{m}}^{*}(\omega)<1 \text { für } 0 \leq \omega \leq \frac{\pi}{\tau}
$$


entsprechend der Bedingung (2.20) für zeitkontinuierliche Systeme. Die Ungleichung (2.29) kann für Systeme von Typ 1 nur dann erfüllt werden, wenn im stationären Zustand die obere Schranke $\bar{l}_{\mathrm{m}}^{*}(\omega)$ der multiplikativen Modellunsicherheit wie im vorliegenden Fall $<1$ ist.

Die obere Schranke $\bar{l}_{\mathrm{m}}^{*}(\omega)$ der multiplikativen Modellunsicherheit wird nach den Gleichungen (2.17) und (2.18) bestimmt, wobei die kontinuierlichen Übertragungsfunktionen durch die diskreten Übertragungsfunktionen ersetzt werden. Unsicherheiten werden bei den zwei Zeitkonstanten $\tau_{\mathrm{m}}$ und $\tau_{\mathrm{lsu}}$ sowie der Totzeit $\tau_{\mathrm{d}}$ angenommen. Die Parameterunsicherheiten von $\tau_{\mathrm{m}}$ und $\tau_{\mathrm{d}}$ resultieren aus den Unsicherheiten und den Verzögerungen bei der Messung der Motordrehzahl und bei der Schätzung des Motorlastmoments. Da hierfür keine genauen Daten vorliegen, wird für alle drei Parameter eine Unsicherheit von $\pm 10 \%$ angenommen.

Die Bedingung für robuste Performance (2.21) kann mit den diskreten Übertragungsfunktionen bei einem quasi zeitkontinuierlichen Reglerentwurf überprüft werden. Da hier jedoch eine Abtastzeit von $10 \mathrm{~ms}$ gewählt wird und die Motorzeitkonstante bei hohen Drehzahlen bis auf $30 \mathrm{~ms}$ absinkt, wird die diskrete Funktionsweise des Regelkreises berücksichtigt. Die Lambdaabweichung $\lambda_{\mathrm{d}}$ kann sich zwischen den Abtastzeitpunkten ändern und daher ist der Zusammenhang zwischen $\lambda_{\mathrm{d}}$ und der Regelabweichung $\lambda_{\mathrm{w}}-\lambda_{\text {lsu }}$ zeitvariant, d.h. es kann keine Störübertragungsfunktion angegeben werden. Morari und Zafiriou [67] nehmen unter der hier gültigen Annahme, dass das Störsignal auf $\pi / \tau$ bandbegrenzt ist, eine Approximation für die Störübertragungsfunktion vor. Nach einigen Rechenschritten erhält man die folgende Bedingung für robuste Stabilität:

$$
|\hat{Q}(\mathrm{j} \omega)| \bar{l}_{\mathrm{a}}(\omega)+|1-\tilde{P}(\mathrm{j} \omega) \hat{Q}(\mathrm{j} \omega)| w(\omega)<1 \text { für } 0 \leq \omega \leq \frac{\pi}{\tau} .
$$

Der Regler $\hat{Q}(\mathrm{j} \omega)$ in Gleichung (2.30) wird nach der Vorschrift

$$
\hat{Q}(\mathrm{j} \omega)=\frac{1}{\tau} H_{0}(\mathrm{j} \omega) \tilde{Q}\left(\mathrm{e}^{\mathrm{j} \omega \tau}\right) F\left(\mathrm{e}^{\mathrm{j} \omega \tau}\right)
$$

berechnet und $w(\omega)$ bezeichnet den Betrag des komplexen Gewichts $w(\mathrm{j} \omega)$, dessen Inverse wie folgt gewählt wird:

$$
w^{-1}(\mathrm{j} \omega)=2,5 \frac{\mathrm{j} \omega+2 \pi 0,1}{\mathrm{j} \omega+2 \pi 0,5} .
$$

Die Parameter von $w$ werden experimentell ermittelt und fordern beim Reglerentwurf, dass im Mitkopplungsbereich die Lambdaabweichung $\lambda_{\mathrm{d}}$ nicht stärker als das 2,5-Fache verstärkt wird und dass die größten Amplituden von $\lambda_{\mathrm{d}}$ bis $0,1 \mathrm{~Hz}$, siehe Bild 2.8, stärker gedämpft werden.

In Bild 2.14 sind die Führungs- und die Störübertragungsfunktion sowie das invertierte Gewicht $w$ für einen Reglerentwurf für die Modellparameter im Motorleerlauf dargestellt. Hier sind die Zeitkonstante und die Totzeit am größten und stellen damit die ungünstigsten Bedingungen dar. Die Zeitkonstante der Lambdasonde wird dabei zu 100 ms gewählt. Die Bandbreite des geschlossenen Regelkreises wird als die Frequenz definiert, bei der der Betrag der Störübertragungsfunktion $G_{\mathrm{d}}$ den Wert $1 / \sqrt{2}$ überschreitet. Beim gewählten Beispiel ergibt sich eine Bandbreite von $0,28 \mathrm{~Hz}$ und damit weniger als die in Abschnitt 2.2.1 gewünschten 0,5 bis $1 \mathrm{~Hz}$. Eine höhere Bandbreite ist jedoch aufgrund der Totzeit in diesem Motorarbeitspunkt nicht realisierbar. Mit steigender Drehzahl sinkt die Totzeit und damit steigt die erzielbare Bandbreite des Lambdaregelkreises. 


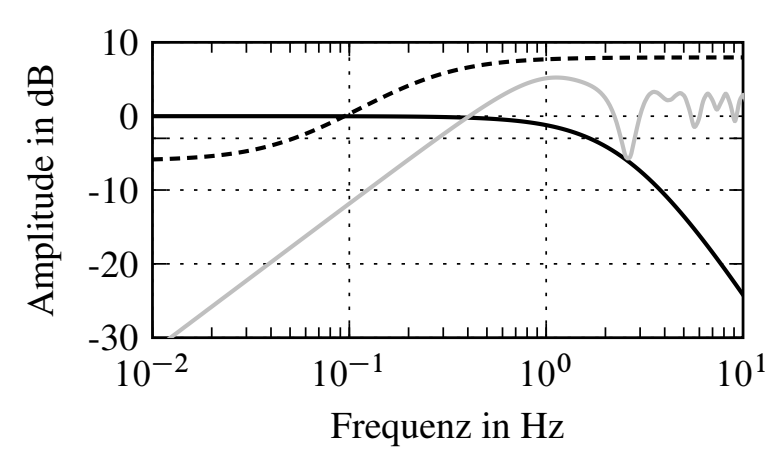

Bild 2.14: Betrag der Führungsübertragungsfunktion $G_{\mathrm{w}}(-)$, der Störübertragungsfunktion $G_{\mathrm{d}}(-)$ und des invertierten Gewichts $w$ (----) für die Modellparameter im Motorleerlauf: $\tau_{\mathrm{m}}=$ $0,36 \mathrm{~s}, \tau_{\mathrm{lsu}}=0,1 \mathrm{~s}$ und $\tau_{\mathrm{d}}=0,28 \mathrm{~s}$

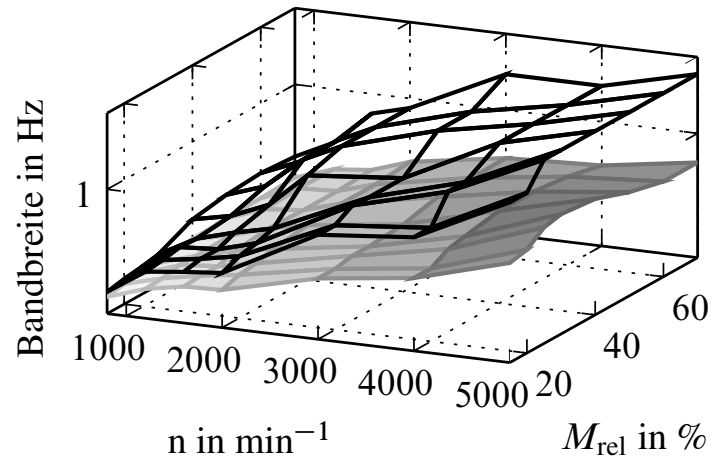

Bild 2.15: Maximale ( $\bigotimes$ ) und aufgrund der Stellgrößenbeschränkung reduzierte $(>)$ Bandbreite des Lambdaregelkreises in Abhängigkeit vom Motorarbeitspunkt

Stellgrößenausnutzung In Anbetracht der Stellgrößenbeschränkung von $\pm 10 \%$ benötigt der Regler $Q(z)$ bei dieser Auslegung relativ hohe Stellgrößen. Bei einem Sprung der Lambdaabweichung $\lambda_{\mathrm{d}}$ von 0 auf $-5 \%$ z. B. überschreitet die Stellgröße die Grenze von $10 \%$ und erreicht einen Maximalwert von etwa $43 \%$. Zwar wird der Regelkreis durch die Implementierung in der IMC-Struktur nicht instabil und es kommt zu keinen Windup-Effekten, es ist dennoch wünschenswert, dass die Stellgröße für Störungen mit kleinen Amplituden nicht in die Stellgrößenbeschränkung geht. In Abschnitt 2.2.1 wird erläutert, dass im hier betrachteten letzten Drittel des FTP 75-Fahrzyklus $95 \%$ der Zeit die Amplitude der Lambdaabweichung kleiner als $5 \%$ ist. Aus diesem Grund und unter der Annahme einer sprungförmigen Störung wird das Filter $F(z)$ noch langsamer ausgelegt, sodass für Amplituden von $\lambda_{\mathrm{d}}$ kleiner als $5 \%$ die Stellgrößenbeschränkung eingehalten wird. Es kann festgehalten werden, dass der zur Verfügung stehende Stellgrößenbereich eine noch stärkere Limitierung der Bandbreite als die Totzeit selbst bewirkt.

Adaption des Reglers Der oben beschriebene Reglerentwurf wird für jeden Motorarbeitspunkt aus den Wertetabellen für die Modellparameter, siehe Bild 2.13, durchgeführt und der Filterparameter $\alpha$ in einer weiteren Wertetabelle gespeichert. In Bild 2.15 ist die maximale, aufgrund der Totzeit limitierte, und die realisierbare, aufgrund der Stellgrößenbeschränkung limitierte, Bandbreite für alle Motorarbeitspunkte dargestellt. Während sich die maximale Bandbreite im Intervall von 0,23 bis 1,45 Hz bewegt, liegt die realisierbare Bandbreite zwischen 0,19 und 0,97 Hz. Das Regelziel, eine Bandbreite von mindesten $0,5 \mathrm{~Hz}$ zu erzielen, kann für Motordrehzahlen erst ab etwa $3000 \mathrm{~min}^{-1}$ erfüllt werden.

Die Modellparameter ändern sich mit dem Motorarbeitspunkt und um diesem Effekt Rechnung zu tragen, wird der Lambdaregler nach einem indirekten Adaptionsschema fortlaufend angepasst. Nach dem „Certainty Equivalence“ Prinzip werden die Modellparameter geschätzt und unter der Annahme, dass sie den wahren Modellparametern entsprechen, wird der IMC-Regler $Q(z)$ neu berechnet [44]. Parameterschätzung und Reglerberechnung können unabhängig voneinander erfol- 


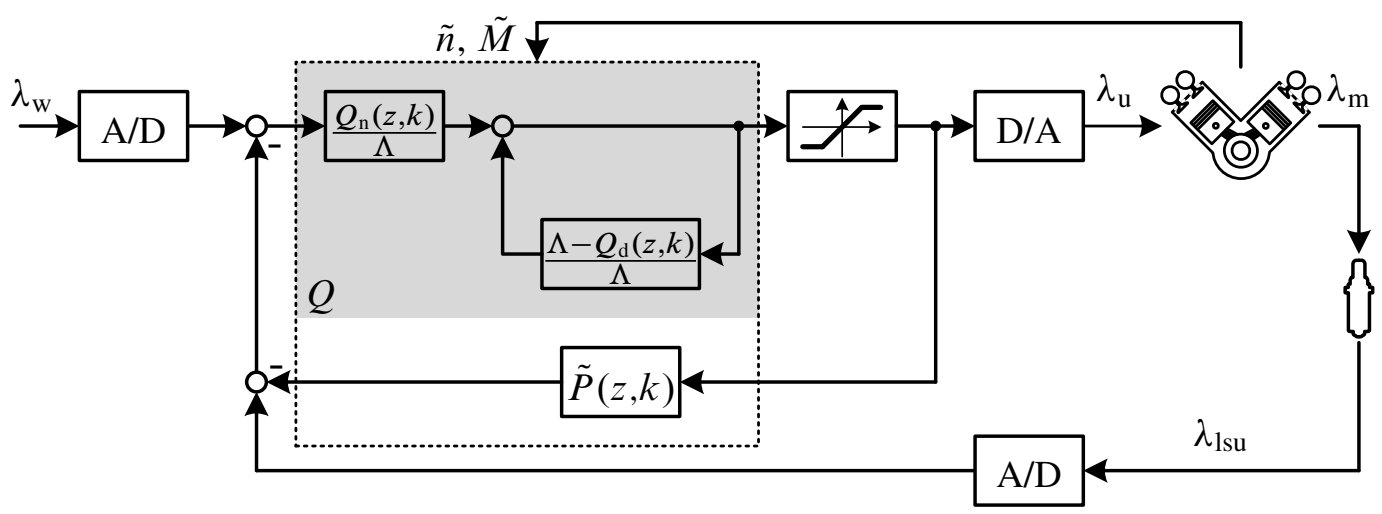

Bild 2.16: Implementierung des adaptiven IMC-Lambdareglers

gen. Da die Modellparameter in Wertetabellen gespeichert sind, kann angenommen werden, dass bereits eine Schätzung für sie vorhanden ist. In Anlehnung an [96] wird die Übertragungsfunktion des IMC-Reglers $Q(z)$ nach der Struktur in Bild 2.16 implementiert. Im Strukturbild bezeichnet $Q_{\text {n }}$ das Zählerpolynom und $Q_{\mathrm{d}}$ das Nennerpolynom der zeitvarianten Reglerübertragungsfunktion $Q(z)$, die zum einen von der komplexen Variable $z$ und zum anderen vom Abtastschritt $k$ abhängt. Des Weiteren bezeichnet $\Lambda(z)$ ein Schur-Polynom. Seine Wurzeln liegen innerhalb des Einheitskreises und daher wird es auch als diskret-stabiles Polynom bezeichnet [35].

Das Adaptionsschema stellt eine Übertragung des zeitkontinuierlichen, adaptiven IMC-Reglerentwurfs in [20] auf den zeitdiskreten Fall dar. Der Ausgangspunkt des Reglerentwurfs ist ein linearparametrisches Modell der zu regelnden Strecke $P$ :

$$
y^{(n)}=\boldsymbol{\Theta}^{\mathrm{T}} \mathbf{u} .
$$

Hierbei bezeichnet $y$ den Modellausgang, $n$ die Modellordnung, der Vektor $\boldsymbol{\Theta}$ die Modellparameter und der Vektor $\mathbf{u}$ enthält die Eingangs- und die Ausgangsgröße sowie ihre ersten $n-1$ Ableitungen. Für diese Modelldarstellung sind im zeitkontinuierlichen Fall die Ableitungen der Ein- und der Ausgangsgröße notwendig, die in der Praxis allerdings nicht immer verfügbar sind. Durch das Filtern der beiden Seiten von Gleichung (2.33) mit einem stabilen Filter $1 / \Lambda$ der Ordnung $n$ können die benötigten Ableitungen durch die gefilterten Werte von $y$ und $u$ ersetzt werden. Um das Ableiten der Stellgröße und der Regelabweichung zu vermeiden, enthält der Regler ebenfalls das Filter $1 / \Lambda$. Der Unterschied zwischen den Implementierungen im zeitkontinuierlichen und im zeitdiskreten Fall ist die Wahl des Polynoms $\Lambda$ : Das Hurwiz-Polynom wird durch ein Schur-Polynom ersetzt. Beim Entwurf des Lambdareglers wird das Filterpolynom zu $\Lambda(z)=z^{2}-z+0,25$ gewählt, sodass die doppelte Polstelle bei 0,5 liegt.

Die Ausführungen in [96] umfassen eine Betrachtung der Stabilität und der Robustheit des diskreten, adaptiven IMC-Reglerentwurfs. Für die Stabilität dieser Reglerimplementierung sprechen folgende Gründe: a) der kontinuierliche Zusammenhang zwischen den Modellparametern und dem Filterparameter $\alpha$; b) der beschränkte Fehler bei der Schätzung der Modellparameter, da diese bereits in Wertetabellen gespeichert sind; sowie c) die begrenzte Änderungsgeschwindigkeit der Modellparameter, da sich die Motordrehzahl nicht sprungförmig ändern kann. 


\subsection{Implementierung des Lambdareglers}

Das zeitkontinuierliche Motormodell in Gleichung (2.22) und der adaptive, zeitdiskrete IMCRegler werden in MATLAB ${ }^{\circledR} /$ Simulink ${ }^{\circledR}$ implementiert und mit den Verläufen der Motorlast, der Motordrehzahl und der Lambdaabweichung in Bild 2.7 simuliert. In Bild 2.17 sind die Verläufe der Zeitkonstante $\tau_{\mathrm{m}}$ und der Totzeit $\tau_{\mathrm{d}}$ des Motormodells, des Filterparameters $\alpha$, der Stellgröße $\lambda_{\mathrm{u}}$ sowie der Regelgröße $\lambda_{\text {lsu }}$ dargestellt. Der Filterparameter folgt in etwa den Verläufen der Modellparameter und macht den Regler langsamer, wenn die Zeitkonstante und die Totzeit steigen. Die Stellgröße bleibt etwa $98 \%$ der Zeit innerhalb der Stellgrößenbeschränkung und es sind keine negativen Effekte zu sehen, wenn die Stellgröße in die Sättigung geht. Im Vergleich zum Verlauf ohne aktive Regelung sorgt der Lambdaregler für die stationäre Genauigkeit und kann vor allem die niederfrequenten Anteile der Lambdaabweichung unterdrücken. Gut zu sehen ist dieses Verhalten im Bereich um $320 \mathrm{~s}$ und ab 470 s. Anhand des zeitlichen Verlaufs ist es, bis auf einige markante Bereiche, jedoch schwierig die Leistungsfähigkeit des Lambdareglers zu beurteilen. Hierfür eignet sich besser das Amplitudenspektrum der Regelgröße, das in Bild 2.18 dargestellt ist. Am Amplitudenspektrum ist gut zu erkennen, dass bis zu einer Frequenz von etwa $0,5 \mathrm{~Hz}$ die Amplitude der Lambdaabweichung reduziert werden kann und ab dieser Frequenz, im Mitkopplungsbereich des Regelkreises, die Amplitude leicht verstärkt wird. Mit weiter steigender Frequenz nimmt diese Verstärkung ab, bis es im Unempfindlichkeitsbereich keinen Unterschied macht, ob der Regler eingesetzt wird oder nicht [59].

Laborprüfstand Zur praktischen Verifikation der in dieser Arbeit entwickelten Regelalgorithmen wird ein Laborprüfstand bei der Firma Umicore verwendet. Eine kurze Beschreibung dieses Prüfstands ist in Anhang A.2 zu finden. Am Laborprüfstand ersetzen Massendurchflussregler zur Dosierung der Gase den realen Motor. Es zeigt sich, dass die Massendurchflussregler durch ein $\mathrm{PT}_{1}$-Glied mit Totzeit modelliert werden können. Daher kann der in diesem Kapitel vorgestellte IMC-Reglerentwurf ohne Änderungen auch für den Laborprüfstand übernommen werden. Mit dem Laborprüfstand können verschiedene Motorarbeitspunkte nachgestellt werden, jedoch kann der gewählte Motorarbeitspunkt aufgrund technischer Beschränkungen nicht dynamisch verändert werden. Aus diesem Grund kann das in der Simulation verwendete Testszenario nicht in dieser Form auch auf den Laborprüfstand übertragen werden. Zu Verifikation des Lambdareglers am Laborprüfstand wird trotz des festen Arbeitspunkts die Lambdaabweichung in Bild 2.17 verwendet und das gleiche Testszenario wird auch in der Simulationsumgebung nachgestellt. Sowohl die Zeitverläufe des Lambdawertes $\lambda_{\text {lsu }}$ als auch die dazugehörigen Amplitudenspektren sind denen in Bild 2.18 sehr ähnlich. Aus diesem Grund wird auf die entsprechende Darstellung an dieser Stelle verzichtet.

\subsection{Zusammenfassung}

In diesem Kapitel werden die wichtigsten Motorkomponenten umrissen und es wird jeweils eine Möglichkeit für ihre Modellierung gezeigt. Die Zwei-Freiheitsgrade-Struktur nach dem Stand 
Zeitkonstante und Totzeit des Motormodells

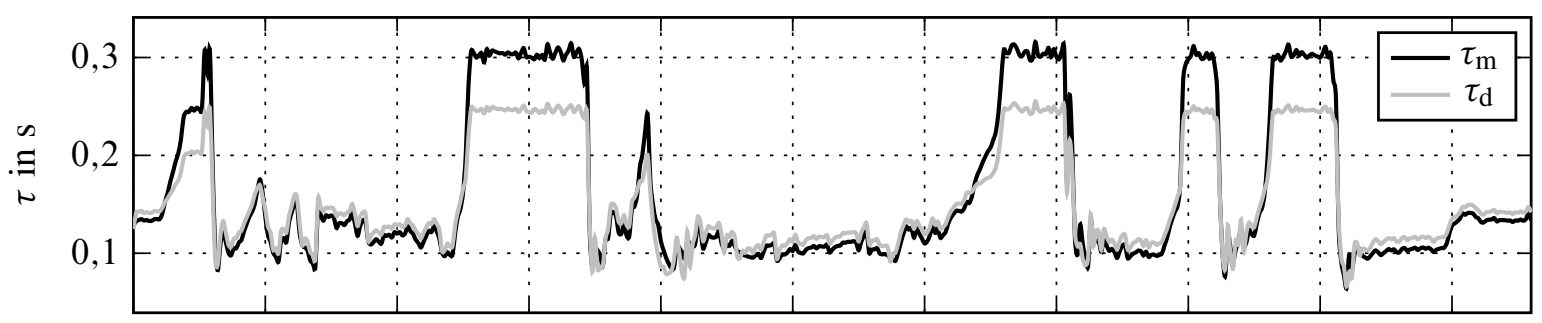

Filterparameter

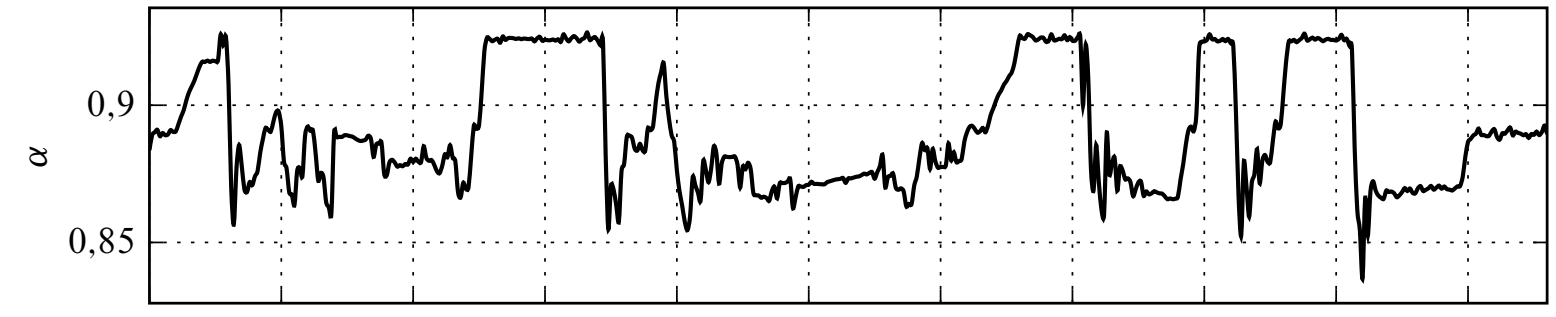

Stellgröße und Regelgröße des Lambdaregelkreises
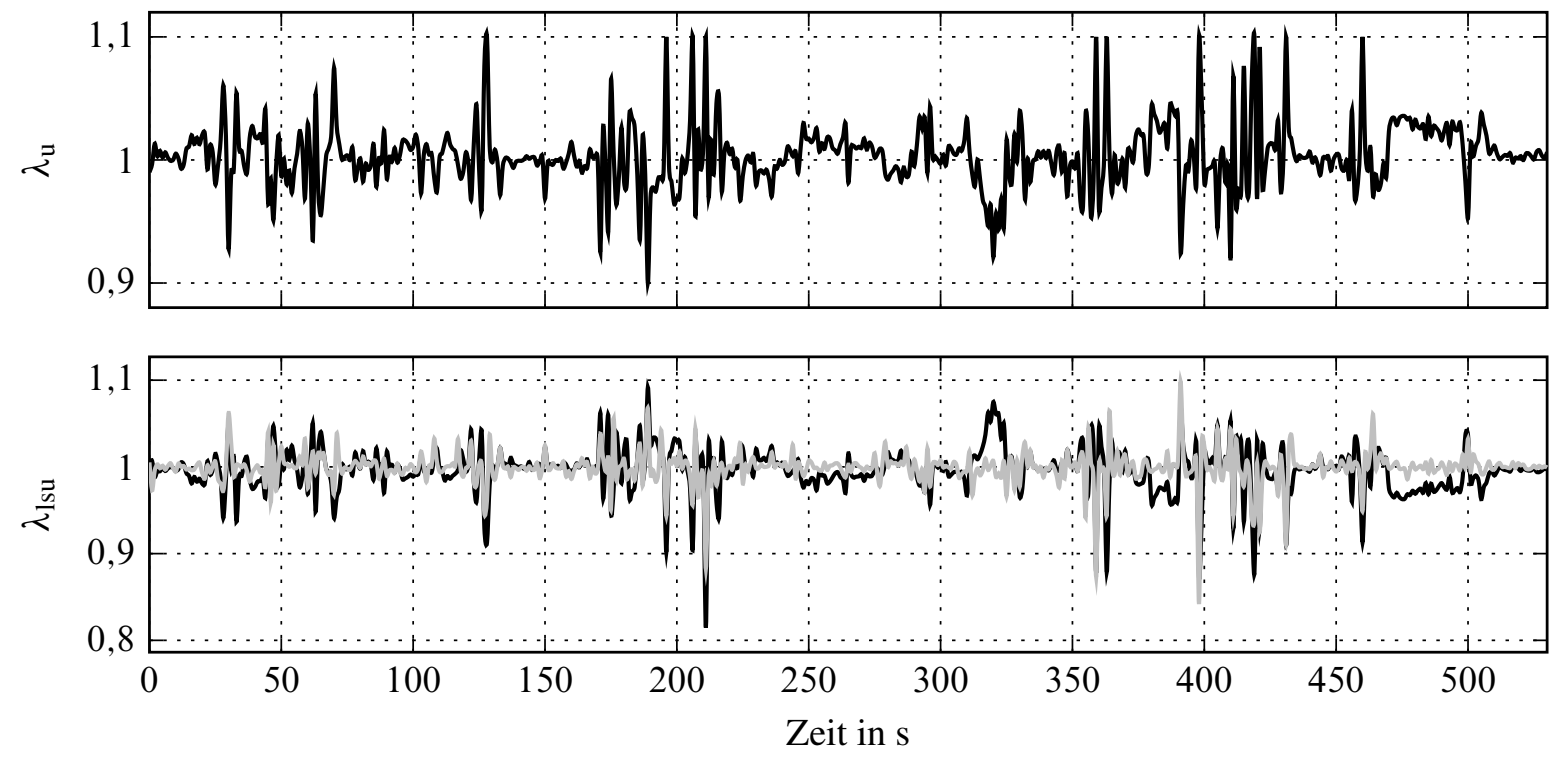

Bild 2.17: Simulation des adaptiven IMC-Lambdareglers mit der von Möller [64] gemessenen Lambdaabweichung $\lambda_{\mathrm{d}}$. Verlauf der Regelgröße ohne (-) und mit Regelung $(-)$.

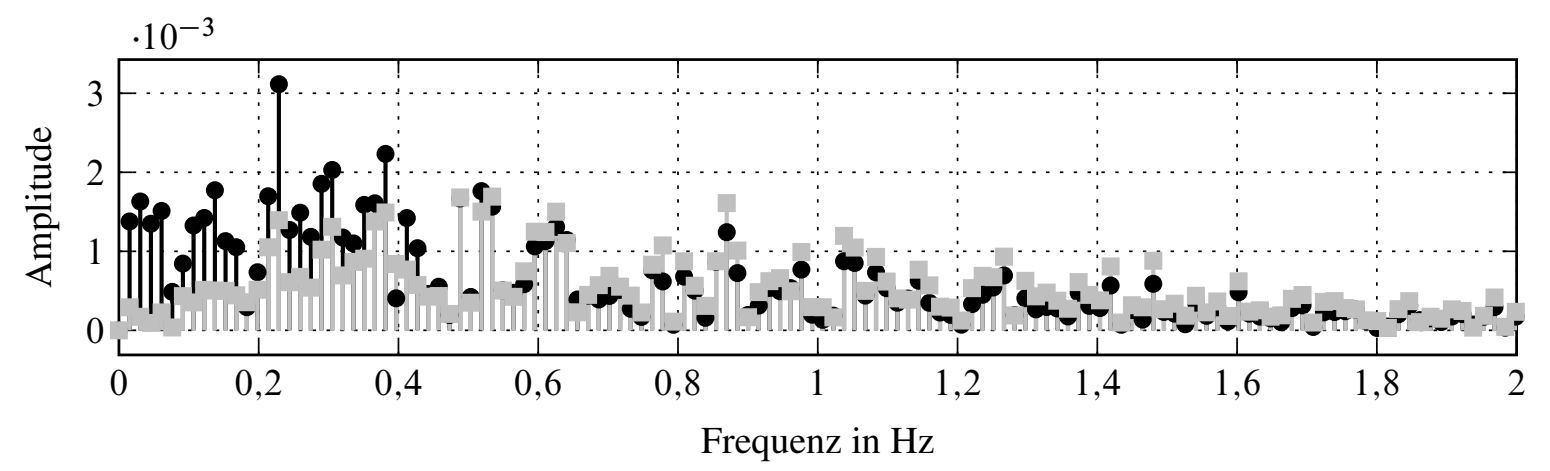

Bild 2.18: Amplitudenspektrum der Regelgröße ohne ( • ) und mit Lambdaregelung ( $\square$ ) 
der Technik besteht aus einer schnellen aber approximativen Vorsteuerung der Kraftstoffeinspritzung und einem Lambdaregler, der für die stationäre Genauigkeit des Lambdaregelkreises sorgt. Die Vorsteuerung wird als vorhanden angenommen und es wird ein diskreter, adaptiver IMCLambdaregler entworfen. Die IMC-Struktur wird aufgrund ihrer Vorteile beim Entwurf und bei der Implementierung des Reglers gewählt, insbesondere bei der vorhandenen Stellgrößenbeschränkung. Beim Reglerentwurf wird neben den Modellunsicherheiten zusätzlich die Ausnutzung der Stellgröße berücksichtigt, sodass der Regler im Betrieb vorwiegend nicht in die Stellgrößenbeschränkung kommt. Der entworfene Regler wird abschließend in der Simulationsumgebung implementiert und getestet. Er zeigt eine gute Leistungsfähigkeit und kann die großen Amplituden der Störung im Frequenzbereich bis etwa $0,5 \mathrm{~Hz}$ reduzieren. Als Störung wird die Lambdaabweichung vom stöchiometrischen Wert eingesetzt, die von Möller [64] beim Nachfahren des letzten Drittels des FTP 75-Fahrzyklus an einem Motorprüfstand bei ausgeschalteter Lambdaregelung aufgenommen worden ist. 


\section{Katalysatormodell}

In diesem Kapitel wird die Modellbildung des Drei-Wege-Katalysators vorgestellt. Das Kapitel beginnt mit einem Überblick über die Katalysatormodelle, die bisher in der Literatur zum modellbasierten Reglerentwurf eingesetzt werden. Anschließend werden die grundsätzliche Vorgehensweise bei der Katalysatormodellierung und die getroffenen vereinfachenden Annahmen erläutert. Der Fokus der Modellbildung in dieser Arbeit liegt auf der Erstellung eines Zustandsraummodells des Katalysators. Auf dem Weg zu seiner Herleitung werden hier die sogenannten Gewichtsfunktionen definiert, welche eine kompakte und intuitive Darstellung des Modells ermöglichen. Ihre Erweiterung auf andere als das hier verwendete Reaktionsschema wird untersucht. Einzelne Aspekte dieses Kapitels sind in Stanchev u. a. [127, 128, 129] zu finden.

Nach Tomforde u. a. [102] können Katalysatormodelle in drei Gruppen eingeordnet werden: detaillierte physikochemische Modelle, vereinfachte physikochemische Modelle und phänomenologische Modelle. Wie in Abschnitt 1.2 diskutiert, eignen sich die Modelle aus der ersten Gruppe wegen ihrer Komplexität nicht zum direkten Einsatz in Regelungssystemen. Diese Modelle sind jedoch wichtig sowohl zur modellbasierten Verifikation der Regelungskonzepte in der Simulationsumgebung als auch zum Verständnis der Reaktionsmechanismen im Katalysator. Für den modellbasierten Regler- und Beobachterentwurf kommen Modelle aus den letzten beiden Gruppen zum Einsatz: phänomenologische Modelle, die vor allem die Dynamik der Sauerstoffspeicherung nachbilden, und vereinfachte physikochemische Modelle, die nur wenige chemische Spezies und Reaktionen berücksichtigen. Damit diese Modelle in Echtzeit auf dem Motorsteuergerät berechnet werden können, werden zahlreiche Vereinfachungen getroffen. Zum Beispiel wird die Dynamik der Temperaturänderungen vernachlässigt, siehe die Arbeit von Guzzella und Onder [40], und obwohl der Katalysator ein System mit örtlich verteilten Parametern darstellt, wird er als System mit konzentrierten Parametern modelliert.

Phänomenologische Modelle Phänomenologischen Modellen liegt die Beobachtung zugrunde, dass das Ein-/Ausgangsverhalten des Katalysators von einem relativ langsamen dynamischen Prozess wie der Sauerstoffspeicherung durch Ceroxid dominiert wird. Diese nichtlineare Speicherdynamik wird durch ein dynamisches Modell nachgebildet und die Gaskonzentrationen hinter dem Katalysator werden, wenn überhaupt, durch statische Kennfelder beschrieben, siehe die Arbeiten von Peyton Jones u. a. [73, 74]. In Bild 3.1 ist die Grundstruktur von so einem phänomenologischen Modell dargestellt. 


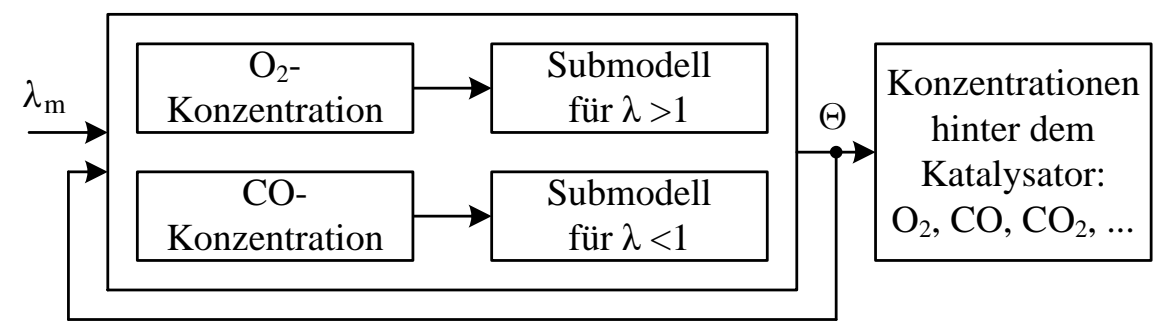

Bild 3.1: Beispielhafte Struktur eines phänomenologischen Katalysatormodells, in Anlehnung an [99]

Einfache Modelle wie diese von Fiengo u. a. [30], Roduner u. a. [85] und Shafai u. a. [90] beschreiben ausschließlich die Dynamik der Sauerstoffspeicherung durch einen beschränkten Integrator. Die Änderung der gespeicherten Sauerstoffmasse ist proportional zum Produkt aus der Lambdaabweichung $\Delta \lambda_{\mathrm{m}}$ im Motorabgas vom stöchiometrischen Lambdawert und dem Abgasmassenstrom $\dot{m}:$

$$
\frac{\mathrm{d}}{\mathrm{d} t} m_{\mathrm{O}_{2}}=0,21 \dot{m} k_{\mathrm{I}}(t) \Delta \lambda_{\mathrm{m}} .
$$

Der Faktor 0,21 in Gleichung (3.1) steht für den Sauerstoffgehalt der Luft und der zeitvariante Faktor $k_{\mathrm{I}}(t)$ berücksichtigt das asymmetrische Verhalten der Sauerstoffspeicherung und der Sauerstoffabgabe, siehe auch die Aufteilung in Bild 3.1, sowie die Temperaturabhängigkeiten und die Verringerung der Sauerstoffspeicherkapazität $L$ infolge der Katalysatoralterung [90]. Der Integratorwert, die gespeicherte Sauerstoffmasse $m_{\mathrm{O}_{2}}$, ist nach unten durch Null und nach oben durch die Sauerstoffspeicherkapazität $L$ begrenzt. Es ist üblich, den zwischen Null und Eins liegenden Sauerstofffüllstand $\Theta$ zu betrachten, den man durch die Skalierung von $m_{\mathrm{O}_{2}}$ mit der Sauerstoffspeicherkapazität $L$ erhält.

Einige Modelle, wie diejenigen von Kako u. a. [48] und Peyton Jones u. a. [74], nutzen als Zustandsgröße nicht den Sauerstofffüllstand $\Theta$, sondern direkt den Lambdawert $\lambda_{\text {su }}$ hinter dem Katalysator ${ }^{1}$. Die Idee dabei ist, dass die schnellen chemischen Reaktionen im Katalysator den ,wirksamen" Lambdawert $\lambda_{\text {su }}$ erfahren, dessen Dynamik durch die Dynamik der Sauerstoffspeicherung vorgegeben wird. Peyton Jones u. a. modellieren die Dynamik durch das folgende sprungfähige System erster Ordnung

$$
\Delta \lambda_{\mathrm{su}}(s)=\frac{b}{a} \frac{s+a}{s+b} \Delta \lambda_{\mathrm{m}} \quad \text { mit } a>b,
$$

da es sich durch Verschieben der Polstelle bei $-b$ gut an magere und fette Arbeitsbereiche anpassen lässt. Die Werte für die Polstelle sind in einer Wertetabelle gespeichert und werden in Abhängigkeit von $\Delta \lambda_{\text {su }}$ eingesetzt. Dadurch ergibt sich ein nichtlineares Modell, das in einem größeren Lambdabereich als die linearen Modelle gültig ist. Für die Ermittlung der Gaskonzentrationen hinter dem Katalysator werden weitere Wertetabellen verwendet. Kako u. a. modellieren die Dynamik zwischen $\Delta \lambda_{\mathrm{m}}$ und $\Delta \lambda_{\mathrm{su}}$ ausschließlich durch ein diskretes $\mathrm{PT}_{1}$-Glied, da von kleinen Abweichungen vom Arbeitspunkt ausgegangen wird. Wie $\Delta \lambda_{\mathrm{m}}$ bezeichnet $\Delta \lambda_{\text {su }}$ die Abweichung vom stöchiometrischen Wert.

\footnotetext{
${ }^{1}$ An dieser Stelle wird die Dynamik der Lambdasonde vernachlässigt.
} 
Je höher bzw. niedriger der Sauerstofffüllstand $\Theta$ ist, umso schwieriger wird die weitere Sauerstoffspeicherung bzw. Sauerstoffabgabe. Um dieses Verhalten in das Modell (3.1) einzubringen, führt Peyton Jones [73] die nichtlineare Funktion $f(\Delta \Theta)$ ein:

$$
\frac{\mathrm{d}}{\mathrm{d} t} \Delta \Theta= \begin{cases}\frac{0,21 \dot{m}}{L} \Delta \lambda_{\mathrm{m}} & \text { für } \Delta \lambda_{\mathrm{m}}<0, \Delta \Theta>0, \\ \frac{0,21 \dot{m}}{L}\left(\Delta \lambda_{\mathrm{m}}-f(\Delta \Theta)\right) & \text { sonst . }\end{cases}
$$

Hierbei bezeichnet $\Delta \Theta$ die Abweichung des Sauerstofffüllstandes $\Theta$ von dem Wert, der sich unter stöchiometrischen Bedingungen einstellt. Die Funktion $f$ verhält sich ähnlich wie eine progressive Feder und wird von Muske und Peyton Jones [69] als Polynom fünfter Ordnung gewählt. Der Lambdawert $\Delta \lambda_{\text {su }}$ hinter dem Katalysator ergibt sich nach der folgenden Gleichung:

$$
\Delta \lambda_{\mathrm{su}}=\Delta \lambda_{\mathrm{m}}-\frac{L}{0,21 \dot{m}} \Delta \dot{\Theta} .
$$

Das Besondere an diesem Modell ist die zusätzliche Modellierung der reversiblen Katalysatordeaktivierung und ihre Auswirkung auf das Signal der Lambdasonde hinter dem Katalysator. Eine ähnliche Struktur weist das Katalysatormodell von Tomforde u. a. auf, allerdings mit dem Unterschied, dass der Katalysator in drei Axialzellen diskretisiert wird.

Wegen der Komplexität der nichtlinearen Vorgänge im Katalysator verfolgen Soumelidis und Stobart [98] den Ansatz, sowohl die Struktur als auch die Parameter des Modells automatisiert zu identifizieren. Zur Modellierung der nichtlinearen Dynamik wird ein Nonlinear AutoRegressive eXogenous (NARX)-Modell angesetzt. Da das reale System als zeitvariant angenommen wird, wird zur Laufzeit eine Anpassung der Struktur und der Parameter des Modells durchgeführt. Die ständige Änderung der Modellstruktur durch den Identifikationsalgorithmus führt dazu, dass eine repräsentative Modellstruktur schwierig zu finden ist. Es ist jedoch zu beobachten, dass bestimmte Terme immer wieder in der Struktur auftauchen und daher mit bestimmten dynamischen Vorgängen zusammenhängen. Im Vergleich zu einem phänomenologischen Modell kann das NARXModell den Lambdawert $\lambda_{\text {su }}$ insbesondere im fetten Bereich besser wiedergeben. Dies kommt jedoch nicht überraschend, da durch die Identifikationsdaten das NARX-Modell auch die reversible Katalysatordeaktivierung berücksichtigt. Soumelidis u. a. [99] greifen die Idee auf, das black-box Modell durch ein gray-box Modell zu ersetzen, indem die Modellstruktur auf Basis einer physikochemischen Modellbildung festgelegt wird.

Vereinfachte physikochemische Modelle Vereinfachte physikochemische Modelle beschreiben ebenfalls die Dynamik der Sauerstoffspeicherung, allerdings besitzen sie im Vergleich zu phänomenologischen Modellen einen direkten Zusammenhang zur Chemie. Daher sind sie ohne Strukturumschaltungen und Kennfelder sowohl für mageres als auch für fettes Abgas gültig. Der Einfachheit halber werden die oxidierenden und die reduzierenden Spezies oft in jeweils eine äquivalente Spezies wie z. B. von Balenovic [5], Bekiaris-Liberis u. a. [9], Guo u. a. [38], Kiwic u. a. [50, 51] und Möller [64] zusammengefasst.

Balenovic sowie Guo u. a. stellen keine expliziten Reaktionsgleichungen auf, sondern nutzen für die Berechnung der Reaktionsgeschwindigkeiten fiktive Reaktionen zwischen dem im Washcoat 
gespeicherten Sauerstoff und dem Sauerstoff bzw. dem Kohlenstoffmonoxid im Motorabgas. Daher können diese Modelle als eine Mischung aus phänomenologischen und physikochemisch motivierten Modellen angesehen werden. Das Modell von Balenovic beschreibt die Änderung des Sauerstofffüllstandes $\Theta$ durch die folgende Gleichung:

$$
\frac{\mathrm{d}}{\mathrm{d} t} \Theta=\frac{1}{k_{\mathrm{d}}(L, \dot{m})}\left(\Delta \lambda_{\mathrm{m}} f(\Theta)+g(\Theta)\right) .
$$

Die Funktion $f(\Theta)$ wird umgeschaltet, je nachdem ob das Motorabgas mager oder fett ist, und die Funktion $g(\Theta)$ beschreibt den Effekt der Speicherung von Kohlenstoffmonoxid und Kohlenwasserstoffen im Washcoat. Der Lambdawert $\lambda_{\mathrm{su}}$ berechnet sich, bis auf die Skalierungsfaktoren, ähnlich wie in Gleichung (3.4). Guo u. a. ermittelt anhand des Sauerstofffüllstandes $\Theta$ noch die $\mathrm{O}_{2^{-}}$, CO-, NO- und HC-Konzentrationen im Abgas hinter dem Katalysator.

Auckenthaler [4], Kiwic u. a. und Möller u. a. [64, 65] stellen jeweils ein Reaktionsschema auf, bei dem die Reaktionsgeschwindigkeiten nach dem Langmuir-Hinshelwood- oder Eley-RidealAnsatz und die Geschwindigkeitskonstanten nach der Arrhenius-Gleichung bestimmt werden. Modellparameter sind unter anderem die präexponentiellen Faktoren, die Aktivierungsenergien und die Änderungen der Freien Enthalpien der Reaktionen. Damit werden die Modellparameter einzelnen Reaktionen und chemischen Spezies zugeordnet und besitzen im Vergleich zu phänomenologischen Modellen eine physikalische Bedeutung. Es ist jedoch nicht zu vergessen, dass die komplexen chemischen Vorgänge im Katalysator nur durch wenige Reaktionen modelliert werden.

Das Reaktionsschema der Sauerstoffspeicherung durch Ceroxid von Möller u. a., das auch in dieser Arbeit verwendet wird, enthält drei reversible Reaktionen. Dahingegen basiert das Modell für den modellbasierten Reglerentwurf von Möller [64] nur auf zwei davon: die Oxidation des Ceroxids durch $\mathrm{O}_{2}$ und die Reduktion des Ceroxids durch CO. Das Modell von Kiwic u. a. ist ähnlich aufgebaut, allerdings mit dem Unterschied, dass Ceroxid durch Platinoxid ersetzt wird und das zusätzlich $\mathrm{CO}_{2}$ als Produkt der Oxidation von $\mathrm{CO}$ enthalten ist. Die Dynamik der Konzentrationen in der Gasphase wird vernachlässigt und nur der Sauerstofffüllstand als dynamische Größe modelliert. Im komplexeren Modell von Auckenthaler werden die Belegungsgrade an $\mathrm{O}_{2}$ und $\mathrm{CO}$ im Washcoat sowie die Temperatur des Trägerkörpers des Katalysators als dynamische Größen modelliert. Neben den $\mathrm{O}_{2}$ - und CO-Gaskonzentrationen wird auch die $\mathrm{H}_{2}$-Konzentration im Abgas hinter dem Katalysator berechnet, da das Signal der Lambdasonde hinter dem Katalysator stark von $\mathrm{H}_{2}$ beeinflusst wird.

Das Katalysatormodell wird von Auckenthaler und Möller in drei und von Kiwic in 20 axiale Zellen diskretisiert. Bekiaris-Liberis u. a. [9] übernehmen das Modell der Sauerstoffspeicherung von Möller u. a. und behalten seinen Charakter als örtlich verteiltes System bei. Die Dynamik der einzelnen Konzentrationen im Washcoat wird vernachlässigt. Unter der Annahme konstanter $\mathrm{CO}_{2-}$ und $\mathrm{H}_{2} \mathrm{O}$-Konzentrationen im Motorabgas verbleiben drei partielle Differentialgleichungen für die $\mathrm{O}_{2-}$, $\mathrm{CO}-$ und $\mathrm{H}_{2}$-Konzentrationen sowie eine partielle Differentialgleichung für den Sauerstofffüllstand $\Theta$. Um das Modell weiter zu vereinfachen, werden die $\mathrm{CO}$ - und $\mathrm{H}_{2}$-Konzentrationen zusammengefasst. 


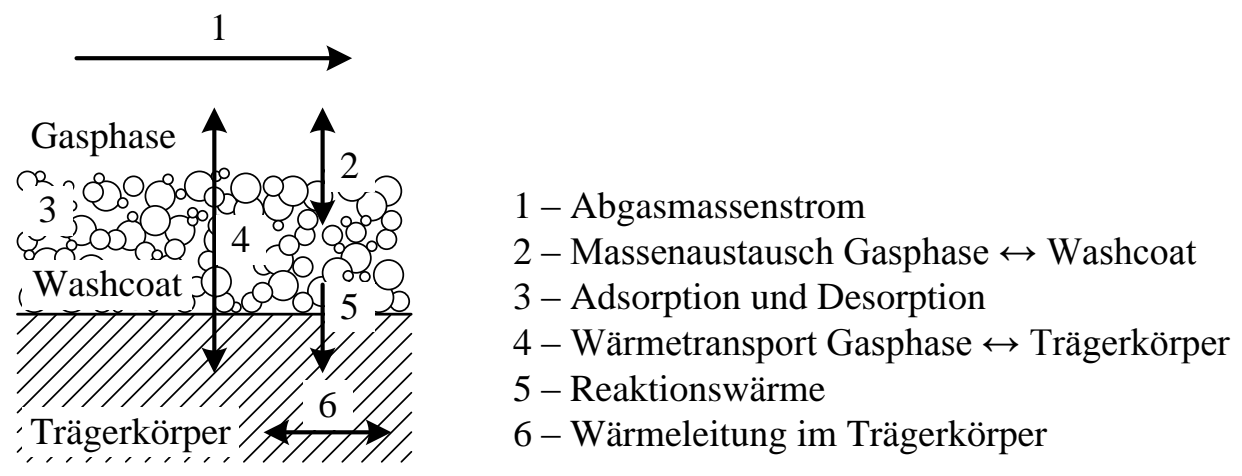

Bild 3.2: Darstellung eines Kanals des Trägerkörpers, in Anlehnung an [64]

\subsection{Modellbildung}

Die Modellbildung des Katalysators kann einerseits in die Modellierung der physikalischen Massentransportprozesse und der thermodynamischen Prozesse und andererseits in die Modellierung des chemischen Reaktionsschemas aufgeteilt werden. Die nachfolgenden zwei Abschnitte widmen sich diesen zwei Themen und orientieren sich an der Vorgehensweise von Möller u. a. [64, $65]$.

Aus Gründen der Geheimhaltung werden die in dieser Arbeit verwendeten Modellparameter nicht explizit angegeben. Dies stellt keine große Einschränkung dar, da der Fokus der Arbeit auf der Methodenentwicklung liegt. Beim Einsatz des Modells in der Praxis wird ein Teil der Parameter aus der Geometrie des Katalysators ermittelt, ein Teil kann aus Tabellen über die Eigenschaften der chemischen Spezies abgelesen werden, siehe z. B. [76], und ein Teil wird experimentell am konkreten Katalysator angepasst, siehe Kapitel 5. In [40], Abschnitt 2.8.3 wird auch das in dieser Arbeit verwendete Reaktionsschema modelliert und in den Tabellen 2.6 und 2.7 können Beispielwerte für die Parametrierung der chemischen Reaktionen gefunden werden. In [9] wird ein Teil der Modellparameter und der Betriebsrandbedingungen angegeben.

\subsubsection{Modellierung der Massentransportprozesse}

Bei der physikalischen Modellbildung ist es üblich, dass ein einzelner Kanal des Trägerkörpers betrachtet wird [4, 16, 64]. ${ }^{2}$ Bei der Modellbildung wird davon ausgegangen, dass am Eingang des Trägerkörpers keine radialen Konzentrations-, Geschwindigkeits- oder Temperaturgradienten vorliegen und keine Wärmeverluste auftreten [16]. In Bild 3.2 sind die drei Phasen im Kanal abgebildet: die Gasphase, der Washcoat und der Trägerkörper.

Gasphase Die physikalischen Größen in der Gasphase sind die Konzentrationen $c_{i}$ der Gasspezies und die Gastemperatur $T_{\mathrm{g}}$. Der Massentransport in der Gasphase wird durch Konvektion und

\footnotetext{
${ }^{2}$ In Abschnitt 1.1.3 wird der Aufbau des Katalysators erläutert.
} 
Diffusion hervorgerufen. Der Massentransport durch axiale Diffusion kann vernachlässigt werden, da er für die typischen Strömungsgeschwindigkeiten in einem Autoabgaskatalysator um den Faktor 50 bis 2500 kleiner als der durch axiale Konvektion hervorgerufene Massentransport ist [16]. Der Kanal wird als ideales Strömungsrohr modelliert und somit angenommen, dass die Konzentrationen der Gasspezies und die Gastemperatur über den Kanalquerschnitt konstant sind und nur von der axialen Koordinate $z$ abhängen. Wie zu erwarten, stellt der Katalysator ein örtlich verteiltes System dar und wird durch partielle Differentialgleichungen beschrieben. Für die Änderung der Konzentration $c_{i}$ jeder Gasspezies $i$ gilt die folgende partielle Differentialgleichung

$$
\frac{\partial}{\partial t} c_{i}=\underbrace{-\frac{\mathrm{R} \dot{m}}{\epsilon_{\mathrm{g}} A_{\mathrm{cs}} p_{\mathrm{exh}} \bar{M}} \frac{\partial c_{i}(z) T_{\mathrm{g}}(z)}{\partial z}}_{\text {axiale Konvektion }}-\underbrace{\frac{k_{\mathrm{c}, \mathrm{i}} A_{\mathrm{geo}}}{\epsilon_{\mathrm{g}}}\left(c_{i}-x_{i}\right)}_{\text {Diffusion Gas } \leftrightarrow \text { Washcoat }},
$$

die die axiale Konvektion durch den Kanal und den Massenaustausch mit dem Washcoat durch Diffusion berücksichtigt. In Gleichung (3.6) bezeichnet $\mathrm{R}$ die universelle Gaskonstante, $\dot{m}$ den Abgasmassenstrom, $\epsilon_{\mathrm{g}}$ den Volumenanteil der Gasphase, $A_{\mathrm{cs}}$ die Querschnittsfläche des Trägerkörpers, $p_{\text {exh }}$ den Abgasdruck, $\bar{M}$ die mittlere molare Masse des Abgases, $k_{\mathrm{c}, i}$ den Massentransportkoeffizienten und $x_{i}$ die Konzentration der jeweiligen Gasspezies im Washcoat.

Durch die Massentransportkoeffizienten $k_{\mathrm{c}, i}$ können auch beim Ansatz eines idealen Strömungsrohres Effekte des radialen Massentransports berücksichtigt werden. Für die Ermittlung der Massentransportkoeffizienten wird die dimensionslose Sherwood-Zahl $S h_{\mathrm{D}}$ verwendet, die als das Verhältnis der effektiv übertragenen Stoffmenge zu der durch Diffusion übertragenen Stoffmenge definiert ist [63]:

$$
S h_{\mathrm{D}}=\frac{k_{\mathrm{c}, i} D_{\mathrm{chan}}}{D_{i, \mathrm{~N}_{2}}} .
$$

In Gleichung (3.7) bezeichnet $D_{\text {chan }}$ den Durchmesser des Kanals und $D_{i, \mathrm{~N}_{2}}$ den binären Diffusionskoeffizienten, der die Diffusion in einem Gemisch aus $\mathrm{N}_{2}$ und der Gasspezies $i$ charakterisiert. Für die Sherwood-Zahl lässt sich kein allgemeingültiger Ausdruck angeben und es werden vielmehr Korrelationen verwendet [16]. In [4, 64] wird für $S h_{\mathrm{D}}$ ein Wert von 2,47 angegeben. Der Diffusionskoeffizient $D_{i, \mathrm{~N}_{2}}$ wird nach der folgenden Vorschrift berechnet [76]

$$
D_{i, \mathrm{~N}_{2}}=\frac{143 \sqrt{\frac{1}{M_{i}}+\frac{1}{M_{\mathrm{N}_{2}}}}}{\sqrt{2}\left(\sum_{v, i}^{1 / 3}+\sum_{v, \mathrm{~N}_{2}}^{1 / 3}\right)^{2} p_{\mathrm{exh}}} T_{\mathrm{g}}^{1,75}
$$

mit der molaren Masse $M$ und dem atomaren Diffusionsvolumen $\sum_{v}$ der Spezies. Für die in dieser Arbeit relevanten Spezies können $M$ und $\sum_{v}$ z. B. [76] entnommen werden.

Washcoat Die physikalischen Größen im Washcoat umfassen neben den Konzentrationen $x_{i}$ der Gasspezies die Konzentrationen bzw. die Belegungsgrade $\Theta_{s}$ der Oberflächenspezies und die Temperatur des Washcoats, die gleich der Temperatur $T_{\mathrm{s}}$ des Trägerkörpers angenommen wird. Für die Änderung der Konzentration $x_{i}$ jeder Gasspezies $i$ gilt die folgende partielle Differential- 
gleichung

$$
\frac{\partial}{\partial t} x_{i}=\frac{D_{\mathrm{chan}}}{4 D_{\mathrm{wc}} \epsilon_{\mathrm{wc}}}(\underbrace{\frac{k_{\mathrm{c}, i} A_{\mathrm{geo}}}{\epsilon_{\mathrm{g}}}\left(c_{i}-x_{i}\right)}_{\text {Diffusion Gas } \leftrightarrow \text { Washcoat }}+\underbrace{\frac{A_{\mathrm{cat}} L}{\epsilon_{\mathrm{g}}} \dot{s}_{i}}_{\text {Quellterm }}),
$$

die den Diffusionsterm in Gleichung (3.6) und einen Quellterm berücksichtigt, der die Erzeugung bzw. Vernichtung der Spezies beschreibt. In Gleichung (3.9) steht $D_{\text {wc }}$ für die Dicke des Washcoats, $\epsilon_{\mathrm{wc}}$ für seine Porosität und $\dot{s}_{i}$ für den Reaktionsquellterm.

Die Änderung des Belegungsgrades $\Theta_{s}$ einer der Oberflächenspezies $s$ entspricht dem Reaktionsquellterm $\dot{S}_{s}$ :

$$
\frac{\partial}{\partial t} \Theta_{s}=\dot{s}_{s}
$$

Temperaturen Für die Änderungen der Temperaturen $T_{\mathrm{g}}$ und $T_{\mathrm{s}}$ können zwei weitere partielle Differentialgleichungen aufgestellt werden [4, 64]. Die Berechnung der Temperaturen kann in ein Submodell ausgelagert werden, das die Reaktionsquellterme $\dot{s}$ zur Berücksichtigung der Reaktionswärme benötigt. Unter der Annahme, dass die Dynamik der Temperaturänderungen langsamer als die Dynamik der Sauerstoffspeicherung ist und dass das Motorsteuergerät Schätzwerte für die Temperaturen liefert, wird das Submodell für die Temperaturen nicht weiter betrachtet. Es wird vielmehr angenommen, dass die Temperaturen $T_{\mathrm{g}}$ und $T_{\mathrm{s}}$ sowie der Abgasmassenstrom $\dot{m}$ die restlichen Modellgleichungen parametrieren. Messungen am Laborprüfstand ergeben, dass sich die Temperaturen im geregelten Katalysatorbetrieb nicht sehr schnell ändern, was die getroffene Annahme unterstützt. Bei Bedarf kann jedoch auch das Submodell für die Temperaturberechnung implementiert werden, ohne Auswirkung auf die weitere Vorgehensweise bei der Modellbildung des Katalysators in diesem Kapitel.

\subsubsection{Modellierung der chemischen Reaktionen}

Das Reaktionsschema zur Beschreibung der Sauerstoffspeicherung durch Ceroxid, das von Möller u. a. $[64,65]$ vorgestellt wird, umfasst die folgenden drei globalen Reaktionsgleichungen

$$
\begin{aligned}
\mathrm{CO}_{2}+\mathrm{Ce}_{2} \mathrm{O}_{3} & \rightleftharpoons \mathrm{CO}+\mathrm{Ce}_{2} \mathrm{O}_{4}, \\
\mathrm{H}_{2} \mathrm{O}+\mathrm{Ce}_{2} \mathrm{O}_{3} & \rightleftharpoons \mathrm{H}_{2}+\mathrm{Ce}_{2} \mathrm{O}_{4}, \\
\mathrm{O}_{2}+2 \mathrm{Ce}_{2} \mathrm{O}_{3} & \rightarrow 2 \mathrm{Ce}_{2} \mathrm{O}_{4}
\end{aligned}
$$

mit den fünf Gasspezies $\mathrm{CO}_{2}, \mathrm{H}_{2} \mathrm{O}, \mathrm{O}_{2}, \mathrm{CO}$ und $\mathrm{H}_{2}$ sowie Ceroxid in seiner reduzierten $\left(\mathrm{Ce}_{2} \mathrm{O}_{3}\right)$ und oxidierten $\left(\mathrm{Ce}_{2} \mathrm{O}_{4}\right)$ Form als Oberflächenspezies. Bei der Betrachtung globaler Reaktionen werden die einzelnen Schritte bei der Adsorption der Reaktanten an der katalytischen Oberfläche, die Reaktion und die anschließende Desorption der Produkte in einer Reaktionsgleichung zusammengefasst. 
Da der gesamte Belegungsgrad an Ceroxid identisch Eins ist, gilt für den Belegungsgrad an reduziertem Ceroxid

$$
\Theta_{\mathrm{Ce}_{2} \mathrm{O}_{3}}=1-\Theta
$$

mit $\Theta$ im Folgendem als Abkürzung für $\Theta_{\mathrm{Ce}_{2} \mathrm{O}_{4}}$. $\Theta$ bezeichnet gleichzeitig den von der axialen Koordinate $z$ abhängigen Sauerstofffüllstand des Katalysators. Die Rückwärtsreaktion in der dritten Reaktionsgleichung (R3) kann vernachlässigt werden, da das chemische Gleichgewicht weit auf der rechten Seite liegt. Das Ceroxid kann durch $\mathrm{CO}_{2}, \mathrm{H}_{2} \mathrm{O}$ und $\mathrm{O}_{2}$ oxidiert und durch $\mathrm{CO}$ und $\mathrm{H}_{2}$ reduziert werden. Damit ist dieses Reaktionsschema deutlich komplexer als bei den meisten aus der Literatur bekannten Modellen, die bisher zum modellbasierten Reglerentwurf eingesetzt werden, siehe den Literaturüberblick am Anfang des Kapitels.

Reaktionsgeschwindigkeiten Die Reaktionsquellterme $\dot{s}_{i}$ und $\dot{s}_{s}$, hier gilt $\dot{s}_{S}=\dot{s}_{\mathrm{Ce}_{2} \mathrm{O}_{4}}$, in den Gleichungen (3.9) und (3.10) besitzen die folgende Struktur

$$
\dot{s}_{i / \mathrm{Ce}_{2} \mathrm{O}_{4}}=\sum_{j} v_{i / \mathrm{Ce}_{2} \mathrm{O}_{4}, j} r_{j}\left(\Theta, T_{\mathrm{s}}\right)
$$

mit den vorzeichenbehafteten stöchiometrischen Koeffizienten $v_{i / \mathrm{Ce}_{2} \mathrm{O}_{4}, j}$ der betrachteten Spezies in der Reaktion $j$ und der dazugehörigen Reaktionsgeschwindigkeit $r_{j}$. Die Reaktionsgeschwindigkeit $r_{j}$ lässt sich im Allgemeinen als Produktansatz darstellen. Zum Beispiel kann für die Hinreaktion in der ersten Reaktionsgleichung (R1) die folgende Gleichung aufgestellt werden:

$$
r_{1}\left(\Theta, T_{\mathrm{s}}\right)=k_{1}\left(T_{\mathrm{s}}\right) x_{\mathrm{CO}_{2}}^{\alpha} \Theta_{\mathrm{Ce}_{2} \mathrm{O}_{3}}^{\beta}=k_{1}\left(T_{\mathrm{s}}\right) x_{\mathrm{CO}_{2}}^{\alpha}(1-\Theta)^{\beta} .
$$

In Gleichung (3.13) bezeichnet $k_{1}$ die Geschwindigkeitskonstante der Reaktion. Die Exponenten $\alpha$ und $\beta$ werden als Teilordnungen bezüglich der Reaktanten $x_{\mathrm{CO}_{2}}$ und $\Theta_{\mathrm{Ce}_{2} \mathrm{O}_{3}}$ bezeichnet. Aus der Summe aller Teilordnungen resultiert die Gesamtordnung der Reaktion. Die Teilordnungen haben keinen Bezug zu den stöchiometrischen Koeffizienten der Reaktionsgleichung und sind in der Praxis experimentell zu bestimmen [19]. In Anlehnung an [50, 64] können jedoch die Beträge der stöchiometrischen Koeffizienten als Startwerte für die Teilordnungen eingesetzt werden. In Gleichung (3.13) sind unter dieser Annahme $\alpha$ und $\beta$ gleich Eins, siehe die Reaktionsgleichung (R1). Da im verwendeten Reaktionsschema alle Reaktionen über die Oberflächenspezies ablaufen und in jeder Reaktion nur eine Gasspezies mit einer Oberflächenspezies reagiert, besitzen alle Reaktionsgeschwindigkeiten die folgende Struktur:

$$
r_{j}\left(\Theta, T_{\mathrm{s}}\right)=k_{j}\left(T_{\mathrm{s}}\right) x_{(j)} \Theta_{(j)}^{\beta_{j}} .
$$

Der Index $(j)$ verweist auf die Konzentration $x_{i}$ bzw. den Belegungsgrad $\Theta_{s}$ der Spezies in der Reaktion $j$, wobei $\beta_{j}$ für die Teilordnung der Oberflächenspezies in der Reaktion $j$ steht.

Geschwindigkeitskonstanten Die Temperaturabhängigkeit der Geschwindigkeitskonstanten der meisten Reaktionen kann mit der empirisch gefundenen Arrhenius-Gleichung beschrieben werden. Die Geschwindigkeitskonstante wird als Produkt eines präexponentiellen Faktors $A$ und einer Exponentialfunktion in der folgenden Form dargestellt:

$$
k\left(T_{\mathrm{s}}\right)=A \mathrm{e}^{\frac{-E}{\mathrm{R} T_{\mathrm{s}}}} .
$$




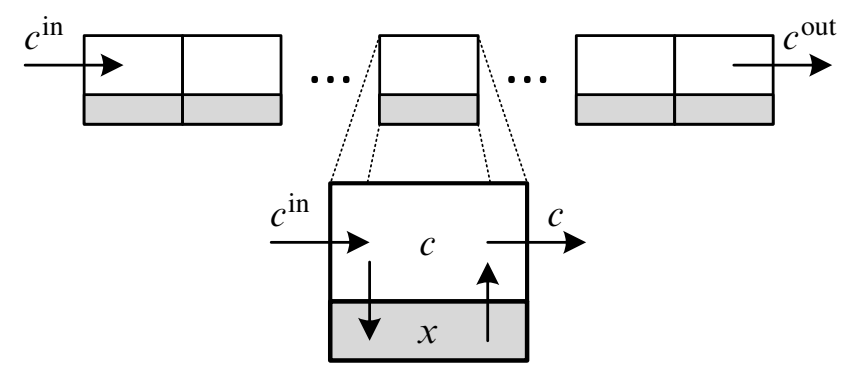

Bild 3.3: Ortsdiskretisierung des eindimensionalen Kanals

Dabei muss ein Teilchen die Aktivierungsenergie $E$ besitzen, um reagieren zu können [19].

Die Geschwindigkeitskonstanten $k^{\mathrm{f}}$ und $k^{\mathrm{b}}$ der Vorwärts- und der Rückwärtsreaktionen können nach der Arrhenius-Gleichung (3.15) wie in $[4,111]$ modelliert werden. Auf diese Weise ist jedoch keine thermodynamische Konsistenz sichergestellt. Dagegen werden in [51, 65] die Geschwindigkeitskonstanten $k^{\mathrm{b}}$ der Rückwärtsreaktionen unter Berücksichtigung der aus thermodynamischen Daten ermittelten Gleichgewichtskonstanten $K$ nach der folgenden Vorschrift berechnet:

$$
k^{\mathrm{b}}\left(T_{\mathrm{s}}\right)=\frac{k^{\mathrm{f}}\left(T_{\mathrm{s}}\right)}{K\left(T_{\mathrm{s}}\right)} .
$$

Dadurch werden die Kinetik und die Thermodynamik der Vorwärts- und der Rückwärtsreaktionen miteinander verknüpft. Die Gleichgewichtskonstanten werden über die Änderung der GibbsEnergie $\Delta G$ berechnet,

$$
K\left(T_{\mathrm{s}}\right)=\mathrm{e}^{-\frac{\Delta G}{R T_{\mathrm{s}}}},
$$

und $\Delta G$ wiederum über die Änderung $\Delta H$ der Enthalpie und $\Delta S$ der Entropie während der Reaktion:

$$
\Delta G\left(T_{\mathrm{s}}\right)=\Delta H\left(T_{\mathrm{s}}\right)-T_{\mathrm{s}} \Delta S\left(T_{\mathrm{s}}\right) .
$$

Die thermodynamischen Parameter $\Delta H$ und $\Delta S$ sind Funktionen der Temperatur $T_{\mathrm{s}}$ und können durch Polynome approximiert werden $[51,65]$.

Ohne Beschränkung der Allgemeinheit werden Gleichgewichtsreaktionen wie (R1) und (R2) im Folgenden in zwei Teilreaktionen aufgeteilt, die der Einfachheit halber als Reaktionen bezeichnet werden. Die Geschwindigkeitskonstanten werden thermodynamisch konsistent ermittelt.

\subsubsection{Vereinfachung der Modellgleichungen}

Wie in Abschnitt 3.1.1 gezeigt, handelt es sich beim Katalysator um ein örtlich verteiltes System und damit um ein unendlichdimensionales System. Um daraus ein Modell endlicher Ordnung zu erhalten, wird der eindimensionale Kanal in axialer Richtung diskretisiert. So entstehen nacheinander geschaltete Zellen, die durch gewöhnliche Differentialgleichungen beschrieben werden, siehe Bild 3.3. Dabei wird jede Zelle als ein idealer Rührkesselreaktor modelliert und somit angenommen, dass innerhalb der Zelle die Konzentrationen und die Temperaturen gleich sind [16]. 
Unter dieser Annahme kann der Term mit der partiellen Ableitung nach $z$ in Gleichung (3.6) wie folgt vereinfacht und anschließend unter Verwendung des Rückwärtsdifferenzenquotienten diskretisiert werden:

$$
\frac{\partial c_{i}(z) T_{\mathrm{g}}(z)}{\partial z} \approx \frac{\partial\left(c_{i} T_{\mathrm{g}}\right)(z)}{\partial z} \approx \frac{c_{i} T_{\mathrm{g}}-c_{i}^{\mathrm{in}} T_{\mathrm{g}}^{\mathrm{in}}}{\Delta z}
$$

Aus Erfahrung ist bekannt, dass die Dynamik der Gasspezies in der Gasphase und im Washcoat wesentlich schneller als die Dynamik der Sauerstoffspeicherung durch Ceroxid ist $[4,51$, 64]. Dies liefert mathematisch ein steifes System von Differentialgleichungen, das schwierig auf einem Motorsteuergerät gelöst werden kann. Abhilfe schafft an dieser Stelle der Ansatz einer quasistationären Gasphase, d.h. $\partial c_{i} / \partial t=0$. Damit wird die Systemdynamik bis auf diese der Sauerstoffspeicherung vernachlässigt. Nach dieser Vereinfachung und nach der Diskretisierung ergeben sich die folgenden algebraischen Gleichungen für eine Zelle des Modells

$$
\begin{aligned}
& 0=-\underbrace{\frac{\mathrm{R} \dot{m}}{A_{\mathrm{cs}} p_{\mathrm{exh}} \bar{M} \Delta z}}_{B_{1}(\dot{m})}\left(c_{i} T_{\mathrm{g}}-c_{i}^{\mathrm{in}} T_{\mathrm{g}}^{\mathrm{in}}\right)-\underbrace{k_{\mathrm{c}, \mathrm{i}} A_{\mathrm{geo}}}_{B_{2, i}\left(T_{\mathrm{g}}\right)}\left(c_{i}-x_{i}\right), \\
& 0=\underbrace{k_{\mathrm{c}, \mathrm{i}} A_{\mathrm{geo}}}_{B_{2, i}\left(T_{\mathrm{g}}\right)}\left(c_{i}-x_{i}\right)+\underbrace{A_{\mathrm{cat}} L}_{B_{3}} \dot{s}_{i}
\end{aligned}
$$

mit $i \in\left\{\mathrm{CO}_{2}, \mathrm{H}_{2} \mathrm{O}, \mathrm{O}_{2}, \mathrm{CO}, \mathrm{H}_{2}\right\}$ und die folgende gewöhnliche Differentialgleichung für die Dynamik des Sauerstofffüllstandes der Zelle:

$$
\frac{\mathrm{d}}{\mathrm{d} t} \Theta=\dot{s}_{\mathrm{Ce}_{2} \mathrm{O}_{4}}
$$

Die Ausdrücke $B_{1}, B_{2, i}$ und $B_{3}$ entstehen durch Zusammenfassen der physikalischen Parameter des Modells und hängen von der Diskretisierung $\Delta z$ und vom Betriebspunkt des Katalysators ab, der sich durch den Abgasmassenstrom $\dot{m}$ und die Temperaturen $T_{\mathrm{g}}^{\text {in }}, T_{\mathrm{g}}$ und $T_{\mathrm{s}}$ bestimmt.

Zusammenfassend besteht das vereinfachte Katalysatormodell pro Zelle aus den algebraischen Gleichungen (3.20) und (3.21), die für jede der fünf Gasspezies aufgestellt werden, und der Differentialgleichung (3.22).

\subsubsection{Emissionsmodell für den Motor}

Die Eingangskonzentrationen $\mathbf{c}^{\text {in }}$ der ersten Zelle des Modells in Bild 3.3 entsprechen den Gaskonzentrationen im Motorabgas, welche vom Motorarbeitspunkt und dem Lambdawert $\lambda_{\mathrm{m}}$ abhängen. Für verschiedene Motordrehzahlen und Motorlastmomente können die Gaskonzentrationen in Abhängigkeit vom Lambdawert messtechnisch erfasst und so ein Emissionsmodell für den Motor erstellt werden. In dieser Arbeit ist experimentell festgestellt worden, dass die Gaskonzentrationen für einen festen Motorarbeitspunkt durch Polynome 4. Ordnung ausreichend genau approximiert werden können. In Bild 3.4 sind die gemessenen und die approximierten Verläufe der Gaskonzentrationen dargestellt. Die Messdaten stammen aus einem Vorgängerprojekt und sind in [64] zu finden. 


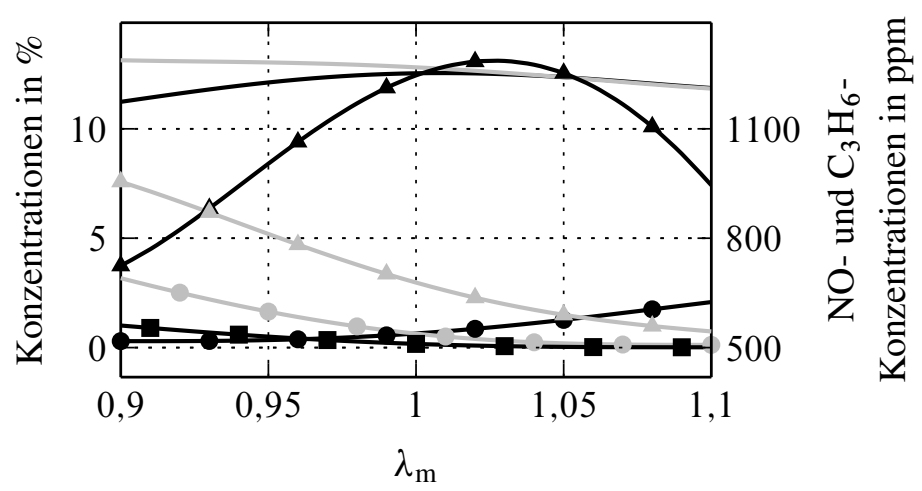

Bild 3.4: Gemessene und approximierte Konzentrationen im rohen Motorabgas am zweiten Motorarbeitspunkt, siehe Anhang A.1: $\mathrm{CO}_{2}(-), \mathrm{H}_{2} \mathrm{O}(-), \mathrm{O}_{2}(-\bullet), \mathrm{CO}(-\bullet), \mathrm{H}_{2}$ $(\rightarrow-), \mathrm{NO}(\neg)$ und $\mathrm{C}_{3} \mathrm{H}_{6}(-)$

Alternativ zu dem hier verwendeten Kennfeld kann auch ein analytisches Emissionsmodell verwendet werden. Dabei werden oft die $\mathrm{CO}_{2}$ - und $\mathrm{H}_{2} \mathrm{O}$-Konzentrationen als konstant angenommen und entweder die $\mathrm{CO}$ - und die $\mathrm{H}_{2}$-Konzentration oder diese von $\mathrm{O}_{2}$ angepasst, je nachdem, ob $\lambda_{\mathrm{m}}$ im fetten oder mageren Bereich liegt [4, 9, 51]. In dieser Arbeit wird die Approximation der Eingangskonzentrationen durch Polynome gewählt, da sich diese einfach ableiten lassen, was im weiteren Verlauf der Arbeit benötigt wird.

Berücksichtigung von Schadstoffen Das Reaktionsschema (R1) bis (R3) enthält fünf Gasspezies, dementsprechend sieht der Vektor mit den Eingangskonzentrationen wie folgt aus:

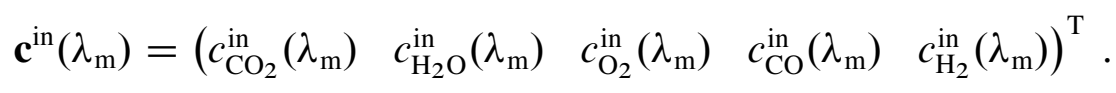

Darin sind Gasspezies im Motorabgas wie z. B. NO und $\mathrm{C}_{3} \mathrm{H}_{6}$ nicht enthalten. Es ist jedoch wichtig, gerade die Schadstoffe zu berücksichtigen, da ihre Menge im Autoabgas reduziert werden soll. Ein erster Ansatz zur Berücksichtigung der im Reaktionsschema nicht vorhandenen Gasspezies ist ihre Umrechnung in eine äquivalente $\mathrm{CO}-, \mathrm{H}_{2}$ - oder $\mathrm{O}_{2}$-Konzentration anhand ihres Sauerstoffbedarfs oder ihres Sauerstoffüberschusses, siehe Tabelle 2.3 in [40].

NO hat ein Sauerstoffatom, das abgegeben werden kann, und kann deshalb durch ein halbes Sauerstoffmolekül repräsentiert werden:

$$
\mathrm{NO} \approx \frac{1}{2} \mathrm{O}_{2}
$$

Jedes Kohlenstoffatom in $\mathrm{C}_{x} \mathrm{H}_{y}$ benötigt zwei Sauerstoffatome, um zu $\mathrm{CO}_{2}$ zu reagieren und wird daher durch die zweifache Menge an $\mathrm{CO}$ repräsentiert, welches selbst nur ein Sauerstoffatom benötigt. Der Wasserstoff in $\mathrm{C}_{x} \mathrm{H}_{y}$ ist bereits als Spezies im Reaktionsschema enthalten, so dass für die Approximation von $\mathrm{C}_{x} \mathrm{H}_{y}$ der folgende Zusammenhang gilt [40]:

$$
\mathrm{C}_{x} \mathrm{H}_{y} \approx 2 x \mathrm{CO} \text { und } \frac{y}{2} \mathrm{H}_{2} .
$$




\subsection{Modelldarstellung mittels Gewichtsfunktionen}

Die Konzentrationen $x_{(j)}$ gehen linear in die Reaktionsgeschwindigkeiten (3.14) ein und damit über die Reaktionsquellterme (3.12) auch linear in die algebraischen Gleichungen (3.21) und die Differentialgleichung (3.22). Damit gehen alle Konzentrationen $c_{i}$ und $x_{i}$ in die Gleichungen (3.20) bis (3.22) linear ein und daher können diese insgesamt 11 Gleichungen durch zwei Matrizengleichungen dargestellt werden. Aus den algebraischen Gleichungen (3.20) und (3.21) resultiert

$$
\mathbf{0}=\mathbf{A}\left(\Theta, T_{\mathrm{s}}, T_{\mathrm{g}}, \dot{m}\right)\left(\begin{array}{l}
\mathbf{c} \\
\mathbf{x}
\end{array}\right)+\mathbf{B}\left(T_{\mathrm{g}}^{\text {in }}, \dot{m}\right) \mathbf{c}^{\text {in }}
$$

und aus der Differentialgleichung (3.22):

$$
\frac{\mathrm{d}}{\mathrm{d} t} \Theta=\mathbf{F}^{\mathrm{T}}\left(\Theta, T_{\mathrm{s}}\right)\left(\begin{array}{l}
\mathbf{c} \\
\mathbf{x}
\end{array}\right) .
$$

Hierbei bezeichnet $\mathbf{c}^{\text {in }}$ den $5 \times 1$-dimensionalen Vektor mit den Eingangskonzentrationen der Zelle gemäß Gleichung (3.23), c den $5 \times 1$-dimensionalen Vektor mit den Gaskonzentrationen in der Zelle und $\mathbf{x}$ den $5 \times 1$-dimensionalen Vektor mit den Konzentrationen im Washcoat, siehe Bild 3.3. Die Reihenfolge der Spezies in den Vektoren entspricht der Festlegung in Gleichung (3.23). $\mathbf{A}$ ist eine $10 \times 10$-dimensionale Matrix und $\mathbf{B}$ eine $10 \times 5$-dimensionale Matrix. Die ersten fünf Elemente des $10 \times 1$-dimensionalen Vektors F sind Null, da der Reaktionsquellterm $\dot{S}_{\mathrm{Ce}_{2} \mathrm{O}_{4}}$ nur von den Konzentrationen $x_{i}$ im Washcoat abhängt. Die restlichen Elemente des Vektors $\mathbf{F}$ resultieren aus dem Zusammenfassen der Terme in Gleichung (3.22), die die gleichen Konzentrationen $x_{i}$ enthalten. Der Übersichtlichkeit halber wird im Folgenden ein Betriebspunkt des Katalysators betrachtet und alle Funktionen nur in Abhängigkeit des Sauerstofffüllstandes $\Theta$ betrachtet.

Die Gleichungen (3.26) und (3.27) beschreiben ein differential-algebraisches System. Die Matrix A in Gleichung (3.26) kann jedoch invertiert werden und die Konzentrationen $\mathbf{c}$ und $\mathbf{x}$ in Abhängigkeit des Sauerstofffüllstandes und der Eingangskonzentrationen berechnet werden:

$$
\left(\begin{array}{l}
\mathbf{c} \\
\mathbf{x}
\end{array}\right)=\underbrace{-\mathbf{A}^{-1}(\Theta) \mathbf{B}}_{\mathbf{G}_{\mathrm{c}, \mathrm{x}}} \mathbf{c}^{\mathrm{in}} \text {. }
$$

In Anhang A.3 wird gezeigt, dass die Matrix A unabhängig von der Parametrierung des Katalysatormodells stets regulär ist. Anschließend kann Gleichung (3.28) in die Differentialgleichung (3.27) eingesetzt werden

$$
\frac{\mathrm{d}}{\mathrm{d} t} \Theta=-\mathbf{F}^{\mathrm{T}}(\Theta) \mathbf{A}^{-1}(\Theta) \mathbf{B} \mathbf{c}^{\mathrm{in}}
$$

und somit das differential-algebraische System aufgelöst werden. Nach Zusammenfassen der Terme in Gleichung (3.29), die die gleichen Eingangskonzentrationen $c_{i}^{\text {in }}$ enthalten, kann die rechte Seite der Differentialgleichung als ein Skalarprodukt dargestellt werden:

$$
\frac{\mathrm{d}}{\mathrm{d} t} \Theta=\underbrace{\left(\begin{array}{lllll}
f_{\mathrm{CO}_{2}}(\Theta) & f_{\mathrm{H}_{2} \mathrm{O}}(\Theta) & f_{\mathrm{O}_{2}}(\Theta) & f_{\mathrm{CO}}(\Theta) & f_{\mathrm{H}_{2}}(\Theta)
\end{array}\right)}_{\mathbf{f}^{\mathrm{T}}(\Theta)} \mathbf{c}^{\mathrm{in}} .
$$




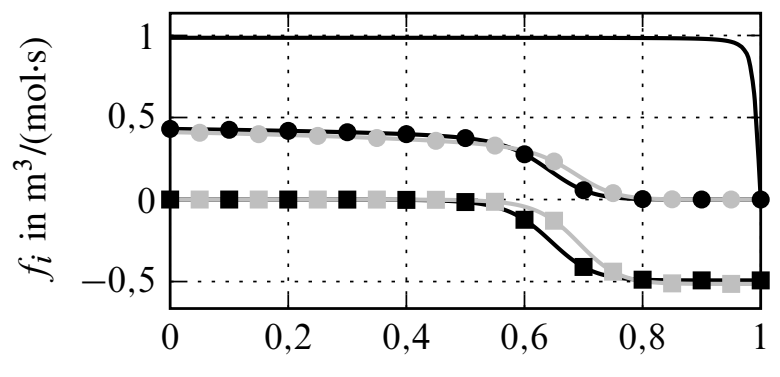

$\Theta$

Bild 3.5: Verlauf der Gewichtsfunktionen am zweiten Motorarbeitspunkt, siehe Anhang A.1: $f_{\mathrm{CO}_{2}}(-\bullet), f_{\mathrm{H}_{2} \mathrm{O}}(-\bullet), f_{\mathrm{O}_{2}}(-), f_{\mathrm{CO}}(\rightarrow-)$ und $f_{\mathrm{H}_{2}}(--)$

Die fünf Elemente des Vektors $\mathbf{f}$ werden in dieser Arbeit Gewichtsfunktionen genannt. Denn jedes Element von $\mathbf{f}$ gewichtet eine Eingangskonzentration $c_{i}^{\text {in }}$ und die Summe aller gewichteten Eingangskonzentrationen ergibt die Änderungsrate des Sauerstofffüllstandes $\Theta$. In Bild 3.5 ist der Verlauf der Gewichtsfunktionen für einen Betriebspunkt des Katalysators dargestellt. Die Gewichtsfunktionen beschreiben quantitativ das Modell, stellen gleichzeitig aber das Katalysatorverhalten auch auf eine anschauliche Weise dar. Die Werte der Gewichtsfunktionen hängen mit der Fähigkeit der Gasspezies zusammen, das Ceroxid zu oxidieren oder zu reduzieren. Bild 3.5 ist deutlich zu entnehmen, dass $\mathrm{O}_{2}$ und bei niedrigen Sauerstofffüllständen auch $\mathrm{H}_{2} \mathrm{O}$ und $\mathrm{CO}_{2}$ den Sauerstoffspeicher befüllen, während $\mathrm{CO}$ und $\mathrm{H}_{2}$ ihn dagegen entleeren. In Kapitel 4 werden die Eigenschaften der Gewichtsfunktionen detaillierter untersucht.

Durch die symbolische Invertierung der Matrix A können die Gewichtsfunktionen nicht nur numerisch sondern auch symbolisch berechnet werden. Zum Beispiel kann die Gewichtsfunktion $f_{\mathrm{CO}_{2}}$ wie folgt dargestellt werden

$$
f_{\mathrm{CO}_{2}}=\frac{p_{1}(1-\Theta)}{p_{2}+\left(p_{3}+p_{4} \mathrm{e}^{\left(p_{5}+p_{6} \Theta^{2}\right)}\right) \Theta}
$$

mit den Funktionen $p_{1}$ bis $p_{6}$, die von den Temperaturen $T_{\mathrm{g}}^{\mathrm{in}}, T_{\mathrm{g}}, T_{\mathrm{s}}$ und dem Abgasmassenstrom $\dot{m}$ abhängen.

Gaskonzentrationen Wie in Bild 3.3 dargestellt, entsprechen die Ausgangskonzentrationen $\mathbf{c}^{\text {out }}$ einer Zelle unter Vernachlässigen der Dynamik der Gasspezies den Gaskonzentrationen in der Zelle selbst und werden durch die obere Hälfte der Matrix $\mathbf{G}_{\mathrm{c}, \mathrm{x}}$ in Gleichung (3.28) beschrieben. Um die Konzentrationen $\mathbf{c}$ zu erhalten, wird daher Gleichung (3.28) von links mit der Matrix $\left(\begin{array}{ll}\mathbf{I}_{5} & \mathbf{0}_{5}\end{array}\right)$ multipliziert:

$$
\mathbf{c}=\underbrace{-\left(\begin{array}{ll}
\mathbf{I}_{5} & \mathbf{0}_{5}
\end{array}\right) \mathbf{A}^{-1}(\Theta) \mathbf{B}}_{\mathbf{G}_{\mathbf{c}}} \mathbf{c}^{\text {in }} .
$$

Hierbei bezeichnet $\mathbf{I}_{5}$ die $5 \times 5$-Einheitsmatrix und $\mathbf{0}_{5}$ die $5 \times 5$-Nullmatrix. Interessant an der Matrix $\mathbf{G}_{\mathrm{c}}$ ist, dass sich ihre Elemente anhand eines affinen Zusammenhangs durch die fünf Gewichtsfunktionen darstellen lassen. Um diesen Zusammenhang herzuleiten, werden die Gleichun- 
gen (3.20) und (3.21) addiert und nach $c_{i}$ umgestellt:

$$
c_{i}=\frac{T_{\mathrm{g}}^{\mathrm{in}}}{T_{\mathrm{g}}} c_{i}^{\mathrm{in}}+\frac{B_{3}}{B_{1} T_{\mathrm{g}}} \dot{s}_{i}
$$

Gemäß den Gleichungen (3.22), (3.12), (3.27) und (3.30) gilt für den Reaktionsquellterm von $\mathrm{Ce}_{2} \mathrm{O}_{4}$ :

$$
\dot{s}_{\mathrm{Ce}_{2} \mathrm{O}_{4}}=\underbrace{\frac{\mathrm{d}}{\mathrm{d} t} \Theta}_{\text {Gl. (3.22) }}=\underbrace{\sum_{j} \hat{r}_{j}}_{\text {Gl. (3.12) }}=\underbrace{\sum_{i} F_{i} x_{i}}_{\text {Gl. (3.27) }}=\underbrace{\sum_{j} f_{(j)} c_{(j)}^{\text {in }}}_{\text {Gl. (3.30) }} .
$$

Die Reaktionsgeschwindigkeiten $\hat{r}_{j}$ in Gleichung (3.34) enthalten die vorzeichenbehafteten stöchiometrischen Koeffizienten $v_{\mathrm{Ce}_{2} \mathrm{O}_{4}, j}$ und $f_{(j)}$ und $c_{(j)}^{\text {in }}$ bezeichnen die mit der Reaktion $j$ zusammenhängende Gewichtsfunktion bzw. Eingangskonzentration. In jeder Reaktion reagiert eine Gasspezies mit einer Oberflächenspezies $\left(\mathrm{Ce}_{2} \mathrm{O}_{3}\right.$ oder $\left.\mathrm{Ce}_{2} \mathrm{O}_{4}\right)$ und definiert die zu dieser Reaktion dazugehörige Gewichtsfunktion, d.h. es existieren so viele Gewichtsfunktionen wie Reaktionsgleichungen.

Die Reaktionsquellterme $\dot{s}_{i}$ der Gasspezies können ebenfalls durch die in Gleichung (3.34) definierten Reaktionsgeschwindigkeiten $\hat{r}_{j}$ dargestellt werden:

$$
\dot{s}_{i}=\sum_{j} v_{i, j} r_{j}=\sum_{j} \frac{v_{i, j}}{v_{\mathrm{Ce}_{2} \mathrm{O}_{4}, j}} \hat{r}_{j}=\sum_{j} \frac{v_{i, j}}{v_{\mathrm{Ce}_{2} \mathrm{O}_{4}, j}} f_{(j)} c_{(j)}^{\mathrm{in}} .
$$

Da jede Gasspezies jeweils nur in einer Reaktion als Reaktant vorkommt, ist sichergestellt, dass die Reaktionsquellterme in Gleichung (3.35) durch die Funktionen $F_{i}$ und schließlich durch die Gewichtsfunktionen $f_{(j)}$ dargestellt werden können. Nach Einsetzen der Reaktionsquellterme (3.35) in Gleichung (3.33) ergibt sich der folgende Zusammenhang für die Gaskonzentrationen in Abhängigkeit der Gewichtsfunktionen:

$$
c_{i}=\underbrace{\frac{T_{\mathrm{g}}^{\mathrm{in}}}{T_{\mathrm{g}}}}_{k_{0}} c_{i}^{\mathrm{in}}+\underbrace{\frac{B_{3}}{B_{1} T_{\mathrm{g}}}}_{k} \sum_{j} \frac{v_{i, j}}{v_{\mathrm{Ce}_{2} \mathrm{O}_{4}, j}} f_{(j)} c_{(j)}^{\mathrm{in}} .
$$

Darin ist der affine Zusammenhang zwischen den Gaskonzentrationen und den Gewichtsfunktionen offensichtlich.

Zur Illustration der Vorgehensweise wird als Beispiel die $\mathrm{CO}_{2}$-Konzentration berechnet. Zuerst wird die Reaktionsgleichung (R1) in die beiden Hinreaktionen

$$
\begin{gathered}
|-1| \mathrm{CO}_{2}+|-1| \mathrm{Ce}_{2} \mathrm{O}_{3} \rightarrow|1| \mathrm{CO}+|1| \mathrm{Ce}_{2} \mathrm{O}_{4}, \\
|-1| \mathrm{CO}+|-1| \mathrm{Ce}_{2} \mathrm{O}_{4} \rightarrow|1| \mathrm{CO}_{2}+|1| \mathrm{Ce}_{2} \mathrm{O}_{3}
\end{gathered}
$$

aufgeteilt, die mit R1.1 und R1.2 bezeichnet werden. Die stöchiometrischen Koeffizienten der Reaktanten haben ein negatives und die der Produkte ein positives Vorzeichen. $\mathrm{CO}_{2}$ ist die reagierende Gasspezies in der ersten Reaktion (R1.1) und damit gehört die Gewichtsfunktion $f_{\mathrm{CO}_{2}} \mathrm{zu}$ dieser Reaktionsgleichung. Analog dazu gehört $f_{\mathrm{CO}}$ zu der zweiten Reaktionsgleichung (R1.2). 
Nach der Vorschrift (3.36) ergibt sich für $c_{\mathrm{CO}_{2}}$ unter Nutzung der Abkürzungen $k_{0}$ und $k$ der folgende Ausdruck:

$$
\begin{aligned}
c_{\mathrm{CO}_{2}} & =k_{0} c_{\mathrm{CO}_{2}}^{\mathrm{in}}+k\left(\frac{v_{\mathrm{CO}_{2}, \mathrm{R} 1.1}}{v_{\mathrm{Ce}_{2} \mathrm{O}_{4}, \mathrm{R} 1.1}} f_{\mathrm{CO}_{2}} c_{\mathrm{CO}_{2}}^{\mathrm{in}}+\frac{v_{\mathrm{CO}_{2}, \mathrm{R} 1.2}}{v_{\mathrm{Ce}_{2} \mathrm{O}_{4}, \mathrm{R} 1.2}} f_{\mathrm{CO}} c_{\mathrm{CO}}^{\mathrm{in}}\right) \\
& =k_{0} c_{\mathrm{CO}_{2}}^{\mathrm{in}}+k\left(\frac{-1}{1} f_{\mathrm{CO}_{2}} c_{\mathrm{CO}_{2}}^{\mathrm{in}}+\frac{1}{-1} f_{\mathrm{CO}} c_{\mathrm{CO}}^{\mathrm{in}}\right) \\
& =k_{0} c_{\mathrm{CO}_{2}}^{\mathrm{in}}-k f_{\mathrm{CO}_{2}} c_{\mathrm{CO}_{2}}^{\mathrm{in}}-k f_{\mathrm{CO}} c_{\mathrm{CO}}^{\mathrm{in}} .
\end{aligned}
$$

Wendet man die gleiche Vorgehensweise zur Berechnung aller Gaskonzentrationen an, so erhält man die Matrizengleichung

$$
\mathbf{c}=\underbrace{\left(\begin{array}{ccccc}
k_{0}-k f_{\mathrm{CO}_{2}} & 0 & 0 & -k f_{\mathrm{CO}} & 0 \\
0 & k_{0}-k f_{\mathrm{H}_{2} \mathrm{O}} & 0 & 0 & -k f_{\mathrm{H}_{2}} \\
0 & 0 & k_{0}-\frac{1}{2} k f_{\mathrm{O}_{2}} & 0 & 0 \\
k f_{\mathrm{CO}_{2}} & 0 & 0 & k_{0}+k f_{\mathrm{CO}} & 0 \\
0 & k f_{\mathrm{H}_{2} \mathrm{O}} & 0 & 0 & k_{0}+k f_{\mathrm{H}_{2}}
\end{array}\right)}_{\mathbf{G}_{\mathbf{c}}} \mathbf{c}^{\text {in }}
$$

und kann daraus die Matrix $\mathbf{G}_{\mathrm{c}}$ ablesen. Auf der Hauptdiagonalen der Matrix ist neben den Gewichtsfunktionen noch die Konstante $k_{0}$ zu finden, die wie ein Durchgriff die Ausgangs- mit den Eingangskonzentrationen direkt verbindet.

Zustandsraummodell Mit den Gleichungen (3.30) und (3.36) kann eine Zelle des Katalysatormodells vollständig durch die Gewichtsfunktionen dargestellt werden. Durch eine Reihenschaltung der Zellen ergibt sich schließlich das nichtlineare Zustandsraummodell für eine beliebige Anzahl von $n_{\mathrm{c}}$ Zellen zu:

$$
\frac{\mathrm{d}}{\mathrm{d} t}\left(\begin{array}{c}
\Theta_{1} \\
\Theta_{2} \\
\vdots \\
\Theta_{n_{\mathrm{c}}}
\end{array}\right)=\left(\begin{array}{c}
\mathbf{f}^{\mathrm{T}}\left(\Theta_{1}\right) \mathbf{c}^{\mathrm{in}} \\
\mathbf{f}^{\mathrm{T}}\left(\Theta_{2}\right) \mathbf{G}_{\mathrm{c}}\left(\Theta_{1}\right) \mathbf{c}^{\mathrm{in}} \\
\vdots \\
\mathbf{f}^{\mathrm{T}}\left(\Theta_{n_{\mathrm{c}}}\right) \mathbf{G}_{\mathrm{c}}\left(\Theta_{n_{\mathrm{c}}-1}\right) \cdots \mathbf{G}_{\mathrm{c}}\left(\Theta_{1}\right) \mathbf{c}^{\mathrm{in}}
\end{array}\right)
$$

Die Ausgangsgleichung des Modells kann je nach Einsatzzweck z. B. als der Lambdawert, eine bestimmte Gaskonzentration oder ein bestimmter Sauerstofffüllstand definiert werden.

\subsection{Erweiterung auf andere Reaktionsschemata}

Wie in Abschnitt 3.1.4 diskutiert, enthält das Reaktionsschema (R1) bis (R3) wichtige Schadstoffe wie z. B. NO und $\mathrm{C}_{3} \mathrm{H}_{6}$ nicht, sodass eine Erweiterung des Reaktionsschemas um weitere Reaktionsgleichungen oder die Nutzung eines anderen Reaktionsschemas vorstellbar ist. Auch in diesem Fall besteht das Interesse, das Katalysatormodell durch Gewichtsfunktionen zu beschreiben und 
ein Zustandsraummodell zu erhalten. Die Herleitung der Gewichtsfunktionen im vorherigen Abschnitt wird zwar für das Reaktionsschema (R1) bis (R3) durchgeführt, da jedoch die Gleichungen allgemein aufgestellt sind, kann die Vorgehensweise auch auf andere Reaktionsschemata übertragen werden. Dabei müssen gewisse Voraussetzungen erfüllt sein, die im Folgenden genannt und an zwei Beispielen veranschaulicht werden.

Reaktionen zwischen Gas- und Oberflächenspezies Der Ausgangspunkt bei der Herleitung der Gewichtsfunktionen ist der lineare Zusammenhang zwischen den Konzentrationen $x_{i}$ im Washcoat und den Reaktionsgeschwindigkeiten $r$. Dieser Zusammenhang ist immer dann gegeben, wenn alle Reaktionen über die Oberflächenspezies ablaufen, d. h. es finden keine Reaktionen direkt zwischen den Gasspezies statt, und die Teilordnung der Gasspezies Eins beträgt. In diesem Fall haben alle Reaktionsgeschwindigkeiten die Struktur in Gleichung (3.13). Wie in Abschnitt 3.1.2 erläutert, hat die Teilordnung einer Spezies keinen Bezug zu ihrem stöchiometrischen Koeffizienten. In den Fällen, in denen die Teilordnung ungleich Eins ist, kann sie eventuell trotzdem auf Eins gesetzt werden und damit ein Fehler bzw. eine Beschränkung des Gültigkeitsbereichs des Modells in Kauf genommen werden. Zum Beispiel werden die Teilordnungen aller Reaktanten von Weilenmann [111] zu Eins gewählt. Des Weiteren werden nur Reaktionen mit zwei Reaktanten betrachtet, sodass jede Gasspezies in maximal einer Reaktion pro Oberflächenspezies als Reaktant vorkommt.

Ein Beispiel für ein Reaktionsschema eines Drei-Wege-Katalysators, das die oben gestellten Bedingungen erfüllt, ist in der Arbeit von Weilenmann zu finden. Das Reaktionsschema umfasst fünf Reaktionsgleichungen, acht Gasspezies und Ceroxid als Oberflächenspezies. Zu den fünf reagierenden Gasspezies können fünf Gewichtsfunktionen definiert werden, während die Gewichtsfunktionen der drei Gasspezies auf der Produktseite der Reaktionsgleichungen identisch Null sind. In Anhang A.4 sind die Reaktionsgleichungen und die Modelldarstellung mittels Gewichtsfunktionen dargestellt.

Reaktionen zwischen Oberflächenspezies Bisher sind nur Reaktionen zwischen einer Gasspezies und einer Oberflächenspezies betrachtet worden. Die Vorgehensweise kann jedoch leicht auch auf Reaktionen erweitert werden, die nur von den Oberflächenspezies abhängen. Ein Beispiel ist die vernachlässigte Rückwärtsreaktion in (R3):

$$
2 \mathrm{Ce}_{2} \mathrm{O}_{4} \rightarrow \mathrm{O}_{2}+2 \mathrm{Ce}_{2} \mathrm{O}_{3}
$$

In diesem Fall und auch bei Reaktionen zwischen Oberflächenspezies hängen die Reaktionsgeschwindigkeiten nicht von den Konzentrationen $x_{i}$ der Gasspezies im Washcoat ab. Daher nimmt Gleichung (3.26) die folgende Form an:

$$
\mathbf{0}=\mathbf{A}_{0}\left(\Theta_{1}\right)+\mathbf{A}\left(\Theta_{1}\right)\left(\begin{array}{l}
\mathbf{c} \\
\mathbf{x}
\end{array}\right)+\mathbf{B} \mathbf{c}^{\mathrm{in}}
$$

Der $2 n_{\mathrm{g}} \times 1$-dimensionale Vektor $\mathbf{A}_{0}$ enthält alle Terme, die unabhängig von den Konzentrationen $x_{i}$ sind, $n_{\mathrm{g}}$ bezeichnet die Anzahl der Gasspezies und $\boldsymbol{\Theta}_{1}$ den Vektor mit den $n_{\mathrm{s}}$ Oberflächenspe- 
zies in der ersten Zelle des Modells. Entsprechend wird auch Gleichung (3.27) modifiziert

$$
\frac{\mathrm{d}}{\mathrm{d} t} \boldsymbol{\Theta}_{1}=\mathbf{F}_{0}\left(\boldsymbol{\Theta}_{1}\right)+\mathbf{F}\left(\boldsymbol{\Theta}_{1}\right)\left(\begin{array}{l}
\mathbf{c} \\
\mathbf{x}
\end{array}\right)
$$

und um den $n_{\mathrm{s}} \times 1$-dimensionalen Vektor $\mathbf{F}_{0}$ erweitert. Analog zu der Vorgehensweise in Abschnitt 3.2 werden die Konzentrationen in der Gasphase und im Washcoat durch die Invertierung der Matrix A in Gleichung (3.40) ermittelt:

$$
\left(\begin{array}{l}
\mathbf{c} \\
\mathbf{x}
\end{array}\right)=\underbrace{-\mathbf{A}^{-1}\left(\boldsymbol{\Theta}_{1}\right) \mathbf{A}_{0}\left(\boldsymbol{\Theta}_{1}\right)}_{\mathbf{G}_{\mathrm{c}, \mathrm{x}, 0}} \underbrace{-\mathbf{A}^{-1}\left(\boldsymbol{\Theta}_{1}\right) \mathbf{B}}_{\mathbf{G}_{\mathrm{c}, \mathrm{x}}} \mathbf{c}^{\mathrm{in}} .
$$

Einsetzen der Konzentrationen $\mathbf{c}$ und $\mathbf{x}$ in die Differentialgleichung (3.41) führt schließlich zu der Auflösung des differential-algebraischen Systems:

$$
\frac{\mathrm{d}}{\mathrm{d} t} \boldsymbol{\Theta}_{1}=\underbrace{\mathbf{F}_{0}\left(\boldsymbol{\Theta}_{1}\right)-\mathbf{F}\left(\boldsymbol{\Theta}_{1}\right) \mathbf{A}^{-1}\left(\boldsymbol{\Theta}_{1}\right) \mathbf{A}_{0}\left(\boldsymbol{\Theta}_{1}\right)}_{\mathbf{f}_{0}} \underbrace{-\mathbf{F}\left(\boldsymbol{\Theta}_{1}\right) \mathbf{A}^{-1}\left(\boldsymbol{\Theta}_{1}\right) \mathbf{B}}_{\mathbf{f}} \mathbf{c}^{\mathrm{in}} .
$$

Der zweite Term auf der rechten Seite der Differentialgleichung (3.43) gewichtet die Eingangskonzentrationen $\mathbf{c}^{\text {in }}$ mit der $n_{\mathrm{s}} \times n_{\mathrm{g}}$-dimensionalen Matrix $\mathbf{f}$, die sich aus den Gewichtsfunktionen $f_{i}^{s}$ zusammensetzt. Der Vektor $\mathbf{f}_{0}$ enthält die Dynamikanteile, die unabhängig von den Eingangskonzentrationen sind.

Die Gaskonzentrationen $\mathbf{c}$ in der Zelle werden wie in Abschnitt 3.2 durch die Multiplikation von Gleichung (3.42) von links mit einer geeigneten Matrix ermittelt:

$$
\mathbf{c}=\mathbf{G}_{\mathrm{c}, 0}\left(\boldsymbol{\Theta}_{1}\right)+\mathbf{G}_{\mathrm{c}}\left(\boldsymbol{\Theta}_{1}\right) \mathbf{c}^{\mathrm{in}} .
$$

Die Matrix $\mathbf{G}_{\mathrm{c}}$ kann wieder durch die Gewichtsfunktionen dargestellt werden, wobei in Gleichung (3.36) für jede Oberflächenspezies ein weiterer Summand hinzugefügt wird. Wie im Falle einer Oberflächenspezies darf auch hier jede Gasspezies maximal einmal mit jeder Oberflächenspezies reagieren.

Mit den Gleichungen (3.43) und (3.44) wird eine Zelle des Modells vollständig beschrieben. Im Unterschied zu Abschnitt 3.2 kann jedoch nur ein Teil des Modells durch Gewichtsfunktionen dargestellt werden. Die Reihenschaltung der Zellen ist durch die Terme $\mathbf{f}_{0}$ und $\mathbf{G}_{\mathrm{c}, 0}$ aufwendiger, aber problemlos möglich und das Zustandsraummodell für eine beliebige Anzahl von $n_{\mathrm{c}}$ Zellen ergibt sich zu:

$$
\frac{\mathrm{d}}{\mathrm{d} t}\left(\begin{array}{c}
\boldsymbol{\Theta}_{1} \\
\boldsymbol{\Theta}_{2} \\
\vdots \\
\boldsymbol{\Theta}_{n_{\mathrm{c}}}
\end{array}\right)=\left(\begin{array}{c}
\mathbf{f}_{0}\left(\boldsymbol{\Theta}_{1}\right) \\
\mathbf{f}_{1}\left(\boldsymbol{\Theta}_{2}, \boldsymbol{\Theta}_{1}\right) \\
\vdots \\
\mathbf{f}_{n_{\mathrm{c}}-1}\left(\boldsymbol{\Theta}_{n_{\mathrm{c}}}, \ldots, \boldsymbol{\Theta}_{1}\right)
\end{array}\right)+\left(\begin{array}{c}
\mathbf{f}\left(\boldsymbol{\Theta}_{1}\right) \mathbf{c}^{\mathrm{in}} \\
\mathbf{f}\left(\boldsymbol{\Theta}_{2}\right) \mathbf{G}_{\mathrm{c}}\left(\boldsymbol{\Theta}_{1}\right) \mathbf{c}^{\mathrm{in}} \\
\vdots \\
\mathbf{f}\left(\boldsymbol{\Theta}_{n_{\mathrm{c}}}\right) \mathbf{G}_{\mathrm{c}}\left(\boldsymbol{\Theta}_{n_{\mathrm{c}}-1}\right) \ldots \mathbf{G}_{\mathrm{c}}\left(\boldsymbol{\Theta}_{1}\right) \mathbf{c}^{\mathrm{in}}
\end{array}\right)
$$

Die Vektorfunktionen $\mathbf{f}_{i}$ mit $i \in\left\{0, \ldots, n_{\mathrm{c}}-1\right\}$ kommen durch das Zusammenfassen aller Terme zustande, die unabhängig von den Konzentrationen der Gasspezies sind. Äquivalent zu Abschnitt 3.2 kann die Ausgangsgleichung des Zustandsraummodells je nach Einsatzzweck gewählt werden. 
Ein Beispiel für ein Reaktionsschema mit mehreren Oberflächenspezies, das die oben gestellten Bedingungen erfüllt, ist in der Arbeit von Scheuer u. a. [88] zu finden. Das Reaktionsschema beschreibt einen Ammoniak-Oxidationskatalysator, der oft mit einem Selective Catalytic Reduction (SCR)-Katalysator kombiniert wird. Es umfasst zehn Reaktionsgleichungen und enthält sechs Gasspezies sowie sechs Oberflächenspezies. Die Belegungsgrade zweier Oberflächenspezies können aus den Belegungsgraden der restlichen vier algebraisch berechnet werden, sodass effektiv nur vier Belegungsgrade numerisch integriert werden müssen. In Anhang A.5 sind die Reaktionsgleichungen und die Modelldarstellung mittels Gewichtsfunktionen dargestellt.

\subsection{Zusammenfassung}

In diesem Kapitel wird die Herleitung eines Zustandsraummodells des Drei-Wege-Katalysators vorgestellt. Ausgehend von den physikalischen Massentransportprozessen werden partielle Differentialgleichungen für die Konzentrationen der Spezies in der Gasphase und im Washcoat aufgestellt. Nach einer Ortsdiskretisierung und unter der Vernachlässigung der schnellen Dynamik der Gasspezies erhält man anschließend ein differential-algebraisches System. Es wird gezeigt, dass unabhängig von der Parametrierung des Modells das differential-algebraisches System aufgelöst werden kann. Nach einigen mathematischen Umformungen kann jede Zelle des Modells vollständig durch fünf Gewichtsfunktionen beschrieben werden. Des Weiteren werden die Anforderungen an das Reaktionsschema untersucht, die die Herleitung der Gewichtsfunktionen ermöglichen. Abschließend wird die Vorgehensweise verallgemeinert und anhand von zwei weiteren Reaktionsschemata beispielhaft vorgeführt. 


\section{Systemanalyse}

In diesem Kapitel wird das nichtlineare Zustandsraummodell des Katalysators mit den Methoden der Regelungstechnik analysiert. Am Anfang des Kapitels wird die Modelldarstellung mittels Gewichtsfunktionen genauer aus den Blickwinkeln der Systemtheorie und der Chemie untersucht. Anschließend wird direkt am nichtlinearen Modell die Systemstabilität untersucht. Für die weiteren Analysen wird das nichtlineare Zustandsraummodell linearisiert. Eine Betrachtung der Verläufe der stationären Arbeitspunkte und der Zeitkonstanten in Abhängigkeit des Lambdawertes oder des Sauerstofffüllstandes gibt Hinweise über die Stärke der Systemnichtlinearitäten. Wesentlicher Aspekt der Systemanalyse ist die Berechnung von Strukturmaßen, die den quantitativen Vergleich verschiedener Katalysatoren ermöglichen. Abschließend wird der Einfluss der Temperatur und des Motorabgasmassenstroms auf die Systemeigenschaften untersucht. Einzelne Aspekte dieses Kapitels sind in Stanchev u. a. [127, 128, 129] zu finden.

In der Literatur sind nur wenige Arbeiten über die Regelung von Drei-Wege-Katalysatoren zu finden, die sich mit der Systemanalyse aus systemtheoretischer Sicht befassen. Eventuell liegt das daran, dass zum einen eine Analyse basierend auf den einfacheren Modellen keine aussagekräftigen Ergebnisse für das reale System liefert und zum anderen die genaueren, aber komplexeren Modelle nicht in Zustandsraumdarstellung vorliegen. Zustandsraummodelle bilden jedoch den Ausgangspunkt vieler modellbasierter Methoden. Auckenthaler [4] schätzt die Stabilität und die Beobachtbarkeit des Katalysators qualitativ ein und empfiehlt eine gründliche Untersuchung dieser Eigenschaften. Balenovic u. a. [7], Fiengo u. a. [30] und Muske und Peyton Jones [69] weisen auf die schlechte Beobachtbarkeit des Sauerstofffüllstandes im Bereich des Lambdafensters hin, während Kiwic u. a. [50, 51] die asymptotische Stabilität der stationären Arbeitspunkte anhand von Simulationsstudien nachweisen.

In der Simulationsumgebung stehen drei Motorarbeitspunkte zur Verfügung, die sich in der Temperatur und dem Massenstrom des Motorabgases unterscheiden, siehe A.1 und [58, S. 1586]. Wenn keine weiteren Angaben gemacht werden, wird für die Simulationen im Folgenden der zweite Motorarbeitspunkt verwendet, der einem Teillastbetrieb des Motors entspricht. An diesem Motorarbeitspunkt beträgt die Abgastemperatur $850 \mathrm{~K}$ und die Raumgeschwindigkeit $24600 \mathrm{~h}^{-1}$. Die Raumgeschwindigkeit ist als das Verhältnis vom Volumenstrom $\dot{V}$ des Motorabgases zum Katalysatorvolumen $V_{\text {cat }}$ definiert:

$$
v_{\mathrm{r}}=\frac{\dot{V}}{V_{\text {cat }}} .
$$

Sie entspricht dem Kehrwert der Verweilzeit des Motorabgases im Katalysator. Die Raumgeschwindigkeit von $24600 \mathrm{~h}^{-1}$ entspricht einer Verweilzeit von etwa $150 \mathrm{~ms}$. 
Bis auf Weiteres wird in dieser Arbeit das Katalysatormodell in drei Zellen diskretisiert. Drei Zellen erlauben die Analyse des Katalysatorverhaltens im vorderen, mittleren und hinteren Bereich, wobei das Modell dennoch übersichtlich bleibt. Auckenthaler geht der Frage nach der günstigen Diskretisierung des Modells nach. Ein Vergleich zwischen Messungen und Simulationen zeigt, dass eine Diskretisierung in drei Zellen in den meisten Testszenarien zufriedenstellende Ergebnisse liefert. Möller [64] und Tomforde u. a. [102] setzen ebenfalls in drei Zellen diskretisierte Katalysatormodelle ein, wohingegen die Diskretisierung in 20 Zellen von Kiwic u. a. eine Ausnahme darstellt.

\subsection{Die Gewichtsfunktionen aus Sicht der Systemtheorie und der Chemie}

Die allgemeine Darstellung eines zeitinvarianten, nichtlinearen dynamischen Systems lautet

$$
\begin{aligned}
\frac{\mathrm{d}}{\mathrm{d} t} \boldsymbol{\Theta} & =\tilde{\mathbf{f}}\left(\boldsymbol{\Theta}, \mathbf{c}^{\mathrm{in}}\right), \\
\mathbf{c} & =\mathbf{g}\left(\boldsymbol{\Theta}, \mathbf{c}^{\mathrm{in}}\right)
\end{aligned}
$$

mit dem Zustandsvektor $\boldsymbol{\Theta}$, dem Eingangsvektor $\mathbf{c}^{\text {in }}$, der Vektorfunktion des Systems $\tilde{\mathbf{f}}$, dem Ausgangsvektor $\mathbf{c}$ und der Ausgangsvektorfunktion g. Die mathematische Handhabung von nichtlinearen Systemen ist viel komplexer als diejenige von linearen Systemen. Deshalb sind bisher nur für bestimmte Klassen nichtlinearer Systeme Methoden zu Analyse und Reglerentwurf entwickelt worden [2]. Viele technische Systeme sind linear bezüglich des Eingangs $\mathbf{c}^{\text {in }}$ und diese Systemklasse ist ausführlich in der Literatur untersucht worden [45]. In Kapitel 3 wird gezeigt, dass unter bestimmten Bedingungen ein Katalysatormodell als ein eingangslineares Zustandsraummodell der Form

$$
\begin{aligned}
\frac{\mathrm{d}}{\mathrm{d} t} \boldsymbol{\Theta}_{1} & =\mathbf{f}_{0}\left(\boldsymbol{\Theta}_{1}\right)+\mathbf{f}\left(\boldsymbol{\Theta}_{1}\right) \mathbf{c}^{\mathrm{in}}, \\
\mathbf{c} & =\mathbf{G}_{\mathrm{c}, 0}\left(\boldsymbol{\Theta}_{1}\right)+\mathbf{G}_{\mathrm{c}}\left(\boldsymbol{\Theta}_{1}\right) \mathbf{c}^{\mathrm{in}}
\end{aligned}
$$

dargestellt werden kann. Der Übersichtlichkeit halber beschreibt das Zustandsraummodell (4.3) nur die erste Zelle des Katalysatormodells. Die Vektorfunktion $\mathbf{f}_{0}$ beschreibt den autonomen Teil des Systems und die Matrix $\mathbf{f}$, in diesem Fall die Matrix mit den Gewichtsfunktionen, beschreibt den Einfluss des Eingangs auf die Systemdynamik. Beim Reaktionsschema von Scheuer u. a. [88], siehe Anhang A.5, finden viele Reaktionen zwischen den Oberflächenspezies statt und daher ist ein wesentlicher Teil der Systemdynamik in der Vektorfunktion $\mathbf{f}_{0}$ enthalten. Die Reaktionsschemata von Möller u. a. [64, 65] und Weilenmann [111], die in Kapitel 3 bzw. Anhang A.4 beschrieben werden, können dagegen vollständig durch Gewichtsfunktionen dargestellt werden. In diesen Fällen ist die Vektorfunktion $\mathbf{f}_{0}$ identisch Null, d.h. diese Modelle sind Spezialfälle der Modelldarstellung (4.3). Da die Modellstruktur (4.3) aus systemtheoretischer Sicht eingehend untersucht worden ist, ist sie der allgemeineren Modellstruktur (4.2) vorzuziehen. Daher bietet die Modelldarstellung mittels Gewichtsfunktionen, sofern möglich, aus Sicht der Systemtheorie die Möglichkeit, die vorhandene Systemstruktur vorteilhaft auszunutzen. 
a)

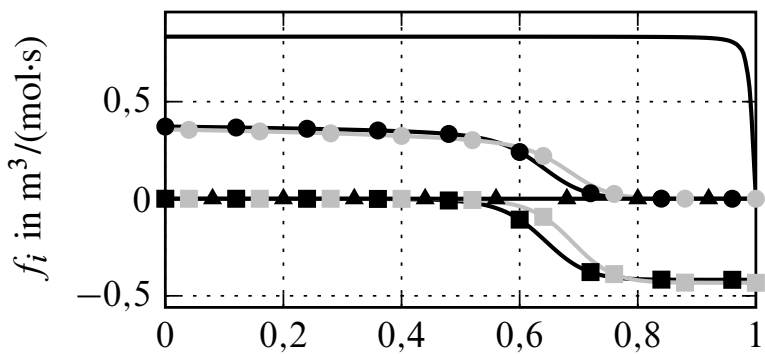

b)

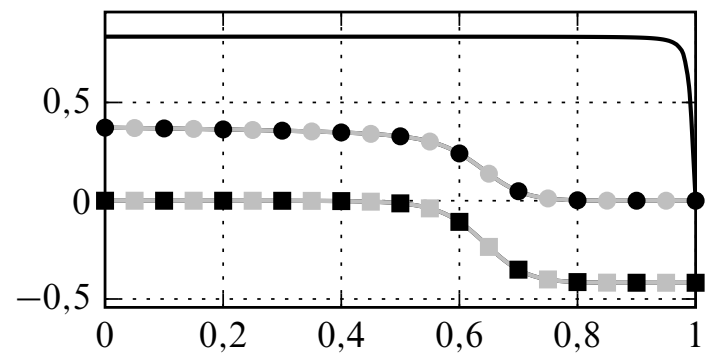

c)

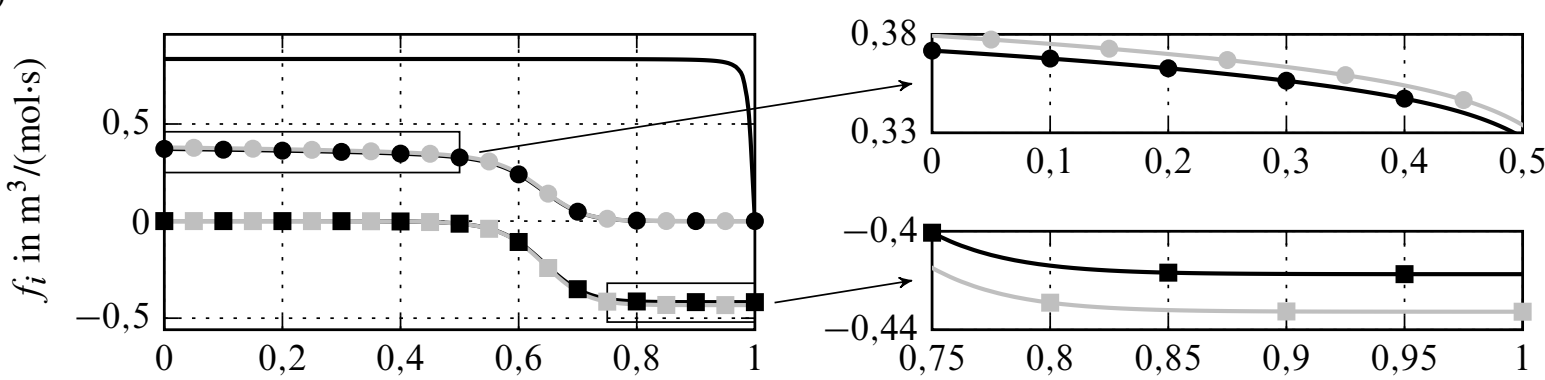

d)

e)

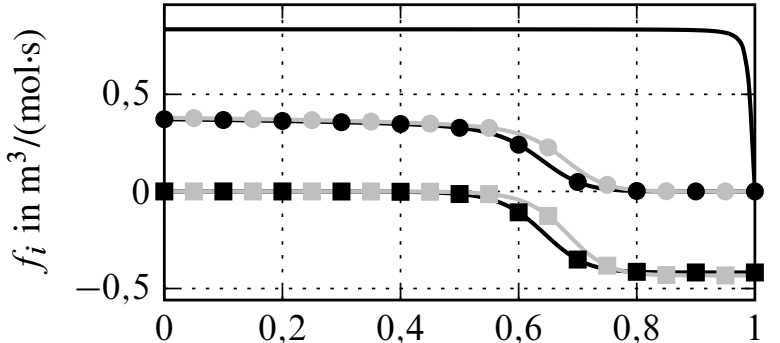

$\Theta$

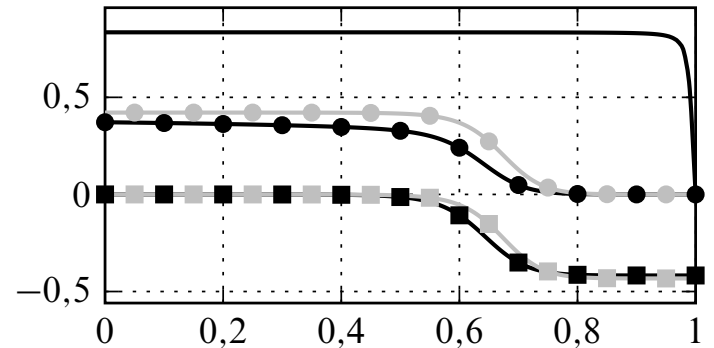

$\Theta$

Bild 4.1: Um den Einfluss der Modellparameter auf die Gewichtsfunktionen in a) zu illustrieren, werden zuerst in b) die physikochemischen Parameter von $\mathrm{H}_{2} \mathrm{O}$ und $\mathrm{H}_{2}$ mit denen von $\mathrm{CO}_{2}$ und $\mathrm{CO}$ gleichgesetzt und anschließend schrittweise auf die Originalwerte zurückgesetzt. In c) werden die Molarmassen und die Diffusionsvolumina zurückgesetzt, in d) die Enthalpie und die Entropie und in e) die Aktivierungsenergie der Reaktion (R2): $f_{\mathrm{CO}_{2}}(\rightarrow-)$, $f_{\mathrm{H}_{2} \mathrm{O}}(-\bullet), f_{\mathrm{O}_{2}}(-), f_{\mathrm{CO}}(-\rightarrow), f_{\mathrm{H}_{2}}(--)$ und $\mathrm{N}_{2}(-)$.

Die Gewichtsfunktionen aus Sicht der Chemie Nach der Betrachtung der Gewichtsfunktionen aus systemtheoretischer Sicht wird im Folgenden eine kurze Analyse aus dem Blickwinkel der Chemie durchgeführt. Um den Einfluss der einzelnen Modellparameter auf die Gewichtsfunktionen besser zu illustrieren, wird ein Gedankenexperiment vorgenommen. Der Ausgangspunkt des Experiments sind die Gewichtsfunktionen in Bild 4.1a, in dem der Vollständigkeit halber neben den fünf Gewichtsfunktionen von $\mathrm{CO}_{2}, \mathrm{H}_{2} \mathrm{O}, \mathrm{O}_{2}, \mathrm{CO}$ und $\mathrm{H}_{2}$ noch die Gewichtsfunktion von $\mathrm{N}_{2}$ dargestellt ist. Im Motorabgas ist $\mathrm{N}_{2}$ zu etwa $72 \%$ vorhanden [36], da es jedoch bei der Modellbildung als Inertgas betrachtet wird, d. h. als Gasspezies, die nicht an der Oxidation oder der Reduktion des Ceroxids beteiligt ist, ist die entsprechende Gewichtsfunktion im gesamten Arbeitsbereich identisch Null. $\mathrm{O}_{2}, \mathrm{H}_{2} \mathrm{O}$ und $\mathrm{CO}_{2}$ oxidieren das Ceroxid und die dazugehörigen Gewichtsfunktionen werden zu Null, wenn das gesamte Ceroxid oxidiert ist. $\mathrm{CO}$ und $\mathrm{H}_{2}$ redu- 
zieren dagegen das Ceroxid und die dazugehörigen Gewichtsfunktionen werden zu Null, wenn das Ceroxid nicht weiter reduziert werden kann. Aufgrund der physikochemischen Eigenschaften der Spezies kann nicht die gesamte Menge an $\mathrm{Ce}_{2} \mathrm{O}_{4}$ bis auf $\mathrm{Ce}_{2} \mathrm{O}_{3}$ reduziert werden. Der Reduktionsgrad hängt von der Temperatur, der Raumgeschwindigkeit des Motorabgases und der Abgaszusammensetzung ab.

Um den Einfluss der einzelnen Modellparameter auf die Gewichtsfunktionen besser zu verstehen, werden im Gedankenexperiment zuerst die Molarmassen $M$, die Diffusionsvolumina $\sum_{v}$, die Enthalpie und die Entropie von $\mathrm{H}_{2} \mathrm{O}$ und $\mathrm{H}_{2}$ mit denen von $\mathrm{CO}_{2}$ und $\mathrm{CO}$ gleichgesetzt. Des Weiteren wird der präexponentielle Faktor sowie die Aktivierungsenergie der Reaktion (R2) mit der von (R1) gleichgesetzt. Dadurch gleichen die Gewichtsfunktionen von $\mathrm{H}_{2} \mathrm{O}$ und $\mathrm{H}_{2}$ denen von $\mathrm{CO}_{2}$ und $\mathrm{CO}$, siehe Bild 4.1b. Durch das sukzessive Wiederherstellen der Originalparameter im Folgenden kann die Form der Gewichtsfunktionen besser verstanden werden.

Zuerst werden die physikalischen Eigenschaften der Spezies zurückgesetzt, d.h. die Molarmassen und die Diffusionsvolumina. Die Molarmassen und die Diffusionsvolumina von $\mathrm{H}_{2} \mathrm{O}$ und $\mathrm{H}_{2}$ nehmen im Vergleich zu denen von $\mathrm{CO}_{2}$ und $\mathrm{CO}$ ab. Die Diffusionskoeffizienten in Gleichung (3.8) steigen an und damit auch die Mobilität dieser Spezies. Der Einfluss von $\mathrm{H}_{2} \mathrm{O}$ und $\mathrm{H}_{2}$ auf das Ceroxid nimmt zu, was an den höheren Absolutwerten der Gewichtsfunktionen in Bild 4.1c zu sehen ist, wenn auch der Einfluss auf die Gewichtsfunktionen sehr gering ist.

Im nächsten Schritt werden die thermodynamischen Eigenschaften der Spezies zurückgesetzt, d. h. die Enthalpie und die Entropie. Die Unterschiede zwischen $\mathrm{H}_{2} \mathrm{O}$ und $\mathrm{CO}_{2}$ bzw. zwischen $\mathrm{H}_{2}$ und $\mathrm{CO}$ nehmen deutlich zu. Offensichtlich haben die thermodynamischen Parameter einen stärkeren Einfluss auf die Eigenschaft von $\mathrm{H}_{2} \mathrm{O}$ und $\mathrm{H}_{2}$, das Ceroxid zu oxidieren bzw. zu reduzieren, siehe Bild 4.1d. Die Gewichtsfunktionen der beiden Spezies bewegen sich in Richtung höherer Sauerstofffüllstände und bedeuten ein höheres Oxidationspotential von $\mathrm{H}_{2} \mathrm{O}$ und ein geringeres Reduktionspotential von $\mathrm{H}_{2}$. Die Erklärung dafür sind die unterschiedlichen Gibbs-Energien der Reaktionen (R1) und (R2). Die Gibbs-Energie bei der Oxidation von $\mathrm{CO}$ zu $\mathrm{CO}_{2}$ beträgt $-208,7 \mathrm{~kJ} \mathrm{~mol}^{-1}$, während diese bei der Oxidation von $\mathrm{H}_{2} \mathrm{zu} \mathrm{H}_{2} \mathrm{O}$ nur $-200,7 \mathrm{~kJ} \mathrm{~mol}^{-1}$ beträgt, d.h. die Rückwärtsreaktion von (R1) hat eine größere Triebkraft als die Rückwärtsreaktion von (R2). Daraus folgt, dass $\mathrm{H}_{2}$ weniger stark als $\mathrm{CO}$ das Ceroxid reduziert und dass $\mathrm{H}_{2} \mathrm{O}$ ein stärkerer Oxidant als $\mathrm{CO}_{2}$ ist. Interessant ist der Schnittpunkt der Gewichtsfunktionen von $\mathrm{H}_{2}$ und $\mathrm{CO}$ bei einem Sauerstofffüllstand von etwa 0,75. Damit ist $\mathrm{H}_{2}$ bei höheren Sauerstofffüllständen ein stärkerer Reduktant als CO. Die Erklärung dafür ist die höhere Mobilität von $\mathrm{H}_{2}$ im Vergleich zu der von CO, siehe Bild 4.1c. Bei höheren Sauerstofffüllständen erreichen die Gewichtsfunktionen von $\mathrm{H}_{2}$ und $\mathrm{CO}$ Plateaus, die durch Limitierungen der Massentransportprozesse zu erklären sind.

Im dritten Schritt wird die Aktivierungsenergie der Reaktion (R2) zurückgesetzt. $\mathrm{H}_{2} \mathrm{O}$ und $\mathrm{H}_{2}$ werden reaktionsfreudiger und infolge dessen vergrößert sich der Abstand zwischen den Gewichtsfunktionen von $\mathrm{H}_{2} \mathrm{O}$ und $\mathrm{CO}_{2}$, während der zwischen $\mathrm{H}_{2}$ und $\mathrm{CO}$ abnimmt, siehe Bild 4.1e. Die Gewichtsfunktion von $\mathrm{H}_{2} \mathrm{O}$ erreicht jetzt für niedrige Sauerstofffüllstände ein Plateau und markiert den Bereich, in dem die Massentransportprozesse limitierend sind. $\mathrm{H}_{2} \mathrm{O}$ oxidiert für alle Sauerstofffüllstände das Ceroxid stärker als $\mathrm{CO}_{2}$, da alle bisher betrachteten physikalischen und 
thermodynamischen Parameter sowie die niedrigere Aktivierungsenergie der Reaktion (R2) dies begünstigen. Die thermodynamischen Eigenschaften machen $\mathrm{H}_{2}$ zu einem schwächeren Reduktanten verglichen mit CO, während die physikalischen Eigenschaften und die Aktivierungsenergie der Reaktion (R2) eine gegensätzliche Wirkung haben. Die niedrigere Aktivierungsenergie der Reaktion (R2) kann die Effekte der Thermodynamik nicht kompensieren, die Limitierungen der Massentransportprozesse sorgen jedoch für einen Schnittpunkt der Gewichtsfunktionen von $\mathrm{H}_{2}$ und $\mathrm{CO}$.

Im letzten Schritt wird der präexponentielle Faktor der Reaktion (R2) zurückgesetzt. Damit wird er um fünf Größenordnungen kleiner und die Reaktionsfreudigkeit von $\mathrm{H}_{2} \mathrm{O}$ und $\mathrm{H}_{2}$ nimmt entsprechend deutlich ab, siehe Bild 4.1a. Der Abstand zwischen den Gewichtsfunktionen von $\mathrm{H}_{2}$ und $\mathrm{CO}$ nimmt zu, wobei das Plateau aufgrund der Massentransportlimitierung bestehen bleibt. $\mathrm{H}_{2} \mathrm{O}$ ist jetzt nur bei höheren Sauerstofffüllständen der stärkere Oxidant. Für Sauerstofffüllstände unter 0,55 ist $\mathrm{CO}_{2}$ der stärkere Oxidant aufgrund der höheren Reaktionsgeschwindigkeit. Interessant ist die Beobachtung, dass für $\mathrm{H}_{2} \mathrm{O}$ und $\mathrm{CO}_{2}$ keine Massentransportlimitierung bei niedrigen Sauerstofffüllständen auftritt und die Gewichtsfunktionen mit sinkendem Sauerstofffüllstand leicht ansteigen.

Dieses Gedankenexperiment zeigt anschaulich, dass auch aus Sicht der Chemie die Gewichtsfunktionen eine Möglichkeit für Systemanalyse bieten. Da in den Gewichtsfunktionen alle physikochemischen Eigenschaften der Spezies enthalten sind, kann der Einfluss einzelner Parameter leicht identifiziert werden, was insgesamt fundierte Vergleiche zwischen einzelnen Spezies und Reaktionen erlaubt. Die Gewichtsfunktionen unterstützen das Verständnis der chemischen Reaktionen und die Identifikation der dominanten Eigenschaften der Reaktionspartner. Nicht zuletzt können solche Gewichtsfunktionen als eine Schnittstelle zwischen der Chemie des Katalysators und seiner Modellierung zum Einsatz in Regelungssystemen dienen.

\subsection{Untersuchung der Stabilität}

Kiwic u. a. [50, 51] untersuchen den Verlauf des Sauerstofffüllstandes entlang des Katalysators für Lambdasprünge am Katalysatoreingang und gehen dabei auf die Eigenschaften der stationären Sauerstofffüllstandsprofile ein. Die durchgeführten Simulationsstudien zeigen, dass es für jeden Lambdawert ein eindeutiges Sauerstofffüllstandsprofil gibt und dass dieses Profil global asymptotisch stabil ist. Das heißt, für einen festen Lambdawert stellt sich unabhängig vom momentanen Sauerstofffüllstand für $t \rightarrow \infty$ das dazugehörige Sauerstofffüllstandsprofil ein.

Phasenraumanalyse Zur anschaulichen Interpretation des Systemverhaltens kann das Zustandsraummodell für eine Phasenraumanalyse eingesetzt werden. Der Übersichtlichkeit halber wird das Katalysatormodell in zwei Zellen diskretisiert und das System im zweidimensionalen Phasenraum betrachtet. Der Phasenraum beschreibt die Menge aller möglichen Zustände des Systems. Das zeitliche Verhalten der Systemzustände kann durch Zustandstrajektorien wiedergegeben werden. Interessant ist die Darstellung des Vektorfeldes der Dynamik, das jedem Zustand die Richtung 
a)

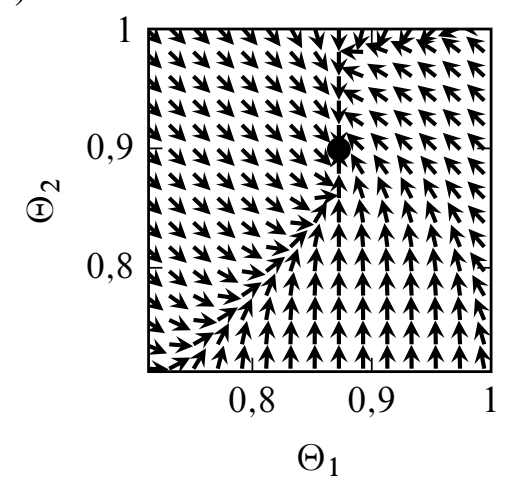

b)

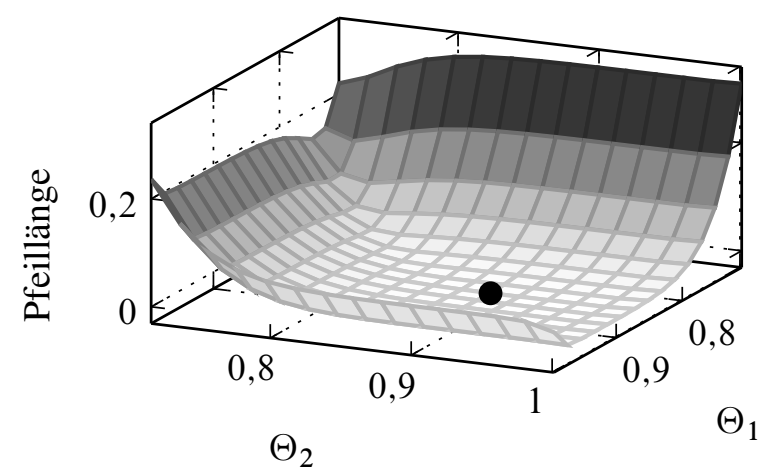

Bild 4.2: Phasenraumanalyse des in zwei Zellen diskretisierten Katalysatormodells für die Ruhelage $\boldsymbol{\Theta}=\left(\begin{array}{ll}0,87 & 0,9\end{array}\right)^{\mathrm{T}}$ : a) skaliertes Vektorfeld; b) Länge der Vektorpfeile

vorgibt, in der sich der Zustand bewegen wird [100]. Zur Erstellung des Vektorfeldes ist die explizite Integration des Zustandsraummodells nicht notwendig, was die Berechnung unabhängig von der Dynamik des Systems macht. Zur Berechnung des Vektorfeldes wird ein stationärer Lambdawert $\lambda_{\mathrm{m}, \infty}$ gewählt, der Phasenraum wird diskretisiert und an jedem diskreten Punkt wird die rechte Seite der Vektordifferentialgleichung

$$
\frac{\mathrm{d}}{\mathrm{d} t}\left(\begin{array}{c}
\Theta_{1} \\
\Theta_{2}
\end{array}\right)=\left(\begin{array}{c}
\mathbf{f}\left(\Theta_{1}\right) \mathbf{c}^{\mathrm{in}}\left(\lambda_{\mathrm{m}, \infty}\right) \\
\mathbf{f}\left(\Theta_{2}\right) \mathbf{G}_{\mathrm{c}}\left(\Theta_{1}\right) \mathbf{c}^{\mathrm{in}}\left(\lambda_{\mathrm{m}, \infty}\right)
\end{array}\right)
$$

ausgewertet. Die Ableitungen an jedem diskreten Punkt legen einen Vektor fest, der die Richtung der Zustandstrajektorie und die Geschwindigkeit der Zustandsänderung wiedergibt. In Bild 4.2a ist das Vektorfeld für einen stationären Lambdawert beispielhaft dargestellt. Es ist leicht zu erkennen, dass aus jedem Anfangszustand die Zustandstrajektorien in die Ruhelage führen und damit die Ruhelage asymptotisch stabil ist. Die Vektorpfeile in Bild 4.2a sind der Übersichtlichkeit halber auf eine einheitliche Länge skaliert, denn aufgrund der Systemnichtlinearitäten ändern sich die Ableitungen der Zustandsgrößen stark in Abhängigkeit des Zustands. Um einen Eindruck davon zu bekommen, ist in Bild 4.2b die Länge der Vektorpfeile dargestellt. Bei Anfangszuständen, die niedrigen Sauerstofffüllständen entsprechen, kommt das System deutlich schneller in die Nähe der Ruhelage als bei Anfangszuständen, die hohen Sauerstofffüllständen entsprechen. In der Nähe der Ruhelage sind die Ableitungen sehr klein, sodass diese nur sehr langsam erreicht wird. Anhand des Vektorfeldes kann die asymptotische Stabilität jedes beliebigen Arbeitspunkts nachgewiesen werden, wobei insgesamt die Systemdynamik bei niedrigen Sauerstofffüllständen höher als diese bei hohen Füllständen ist.

Direkte Methode von Ljapunov Die Stabilitätsanalyse im Phasenraum ist zwar anschaulich, lässt sich jedoch nur für Systeme mit maximal drei Zuständen anwenden und selbst bei drei Zuständen ist es schwierig, das Vektorfeld zu überblicken. Aus diesem Grund wird die direkte Methode von Ljapunov zum Nachweis der asymptotischen Stabilität des Katalysatormodells bei einer Diskretisierung in mehr als zwei Zellen angewendet. Der Vorteil gegenüber der Phasenraumanalyse 
ist, dass alle Schritte der Methode automatisiert ausgeführt werden können und keine qualitativen Betrachtungen wie beim Vektorfeld notwendig sind. Ähnlich wie bei der Berechnung des Vektorfeldes ist für die Anwendung der direkten Methode von Ljapunov die Lösung der Vektordifferentialgleichung (4.2a) nicht nötig. Die direkte Verwendung der Differentialgleichung gibt der Methode ihren Namen [2]. Bei der direkten Methode von Ljapunov wird die Ableitung einer reellwertigen, positiven Funktion $V$ in Richtung der Vektorfunktion $\tilde{\mathbf{f}}$ des Systems betrachtet, d. h. entlang der Zustandstrajektorien. Die zu untersuchende Ruhelage ist genau dann asymptotisch stabil, wenn entlang aller Trajektorien in einer Umgebung $U_{1}$ der Ruhelage die Vektorfunktion $V$ abnimmt, d. h. $\dot{V}<0$ gilt. Die Funktion $V$ muss in der Ruhelage ein Minimum besitzen und in einer Umgebung $U_{0}$, die die Umgebung $U_{1}$ einschließt, stetig sein und stetige partielle $\mathrm{Ab}$ leitungen besitzen. Es wird weiter vorausgesetzt, dass die Vektordifferentialgleichung (4.2a) für jeden Anfangszustand aus der Umgebung $U_{1}$ der Ruhelage eine stetige und eindeutige Lösung besitzt. Für die praktische Anwendung der Methode am Katalysatormodell wird die Ableitung der Ljapunov-Funktion nach der Zeit gebildet

$$
\frac{\mathrm{d}}{\mathrm{d} t} V(\boldsymbol{\Theta}(t))=\frac{\mathrm{d}}{\mathrm{d} t} \boldsymbol{\Theta}^{\mathrm{T}} \cdot \operatorname{grad} V(\boldsymbol{\Theta})
$$

und nach Einsetzen der Vektordifferentialgleichung wird überprüft, ob $\dot{V}(\boldsymbol{\Theta})<0$ in der Umgebung $U_{1}$ gilt. Da der gesamte mögliche Arbeitsbereich als $U_{1}$ definiert wird, wird die globale asymptotische Stabilität überprüft. Wie bei der Phasenraumanalyse wird der Zustandsraum diskretisiert und die Ableitung (4.5) punktuell im Arbeitsbereich berechnet.

Eine Herausforderung bei dem praktischen Einsatz der Methode liegt darin, eine geeignete Ljapunov-Funktion zu finden und oft ist man auf das Ausprobieren verschiedener Funktionsansätze angewiesen [2]. Als Ansatz für Ljapunov-Funktionen werden oft Funktionen mit kreis- oder ellipsoidförmigen Höhenlinien gewählt, die durch die positiv definite quadratische Form

$$
V(\boldsymbol{\Theta})=\boldsymbol{\Theta}^{\mathrm{T}} \mathbf{M} \boldsymbol{\Theta}
$$

definiert sind. Solche Funktionen sind auch für den Nachweis der asymptotischen Stabilität des Katalysatormodells geeignet. Für die Matrix M in Gleichung (4.6) werden Matrizen mit konstanten Einträgen auf der Haupt- und den Nebendiagonalen gewählt. Für eine Diskretisierung des Modells in drei Zellen und die Ruhelage $\boldsymbol{\Theta}=\left(\begin{array}{lll}0,87 & 0,9 & 0,9\end{array}\right)^{\mathrm{T}}$ wird z. B. folgende Matrix $\mathbf{M}$ eingesetzt:

$$
\mathbf{M}=\left(\begin{array}{lll}
4 & 2 & 1 \\
2 & 4 & 2 \\
1 & 2 & 4
\end{array}\right) .
$$

Nach der Berechnung der Ableitung der Funktion $V$, siehe Gleichung (4.5), an den Stützstellen des diskretisierten, dreidimensionalen Zustandsraums, kann die globale asymptotische Stabilität der Ruhelage numerisch nachgewiesen werden. Die Ableitung $\dot{V}$ lässt sich in diesem Fall jedoch nicht anschaulich darstellen, sodass die Methode auch an einem in zwei Zellen diskretisierten Katalysatormodell angewendet wird. Bild 4.3 zeigt ein Beispiel für dieselbe Ruhelage wie in Bild 4.2 . 


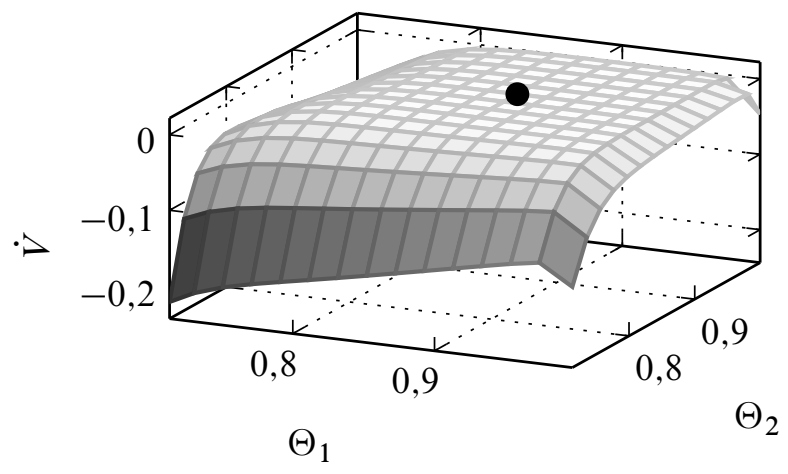

Bild 4.3: Ableitung der Ljapunov-Funktion in Gleichung (4.8)

Die Ableitung der gewählten Ljapunov-Funktion

$$
V(\boldsymbol{\Theta})=2 \Theta_{1}^{2}+2 \Theta_{1} \Theta_{2}+2 \Theta_{2}^{2}
$$

ist in der Nähe der Ruhelage zwar negativ aber sehr nahe an Null. Dies bestätigt die Beobachtung bei der Phasenraumanalyse, dass das autonome System die Ruhelage nur sehr langsam erreicht.

\subsection{Linearisierung des Modells}

Zur weiteren Analyse des Modells werden die Systemgleichungen linearisiert. Bezüglich der Eingangskonzentrationen $\mathbf{c}^{\text {in }}$ ist das Zustandsraummodell (3.39) eingangsaffin, sodass bei der Linearisierung die Gewichtsfunktionen und das Emissionsmodell unabhängig voneinander nach $\Theta$ bzw. $\lambda_{\mathrm{m}}$ differenziert werden können. Da sich die Gewichtsfunktionen und das Emissionsmodell symbolisch darstellen lassen, können sie auch symbolisch differenziert werden, siehe Kapitel 3. ${ }^{1}$ Die Linearisierung des kompletten Katalysatormodells führt zu einer Vektordifferentialgleichung der Form

mit der Systemmatrix

$$
\frac{\mathrm{d}}{\mathrm{d} t} \Delta \boldsymbol{\Theta}=\mathbf{A}_{1} \Delta \boldsymbol{\Theta}+\mathbf{B}_{1} \Delta \lambda_{\mathrm{m}}
$$

$$
\mathbf{A}_{1}=\left.\left(\begin{array}{ccc}
\frac{\partial \mathbf{f}^{\mathrm{T}}\left(\Theta_{1}\right)}{\partial \Theta_{1}} \mathbf{c}^{\mathrm{in}}\left(\lambda_{\mathrm{m}}\right) & 0 & \cdots \\
\mathbf{f}^{\mathrm{T}}\left(\Theta_{2}\right) \frac{\partial \mathbf{G}_{\mathrm{c}}\left(\Theta_{1}\right)}{\partial \Theta_{1}} \mathbf{c}^{\mathrm{in}}\left(\lambda_{\mathrm{m}}\right) & \frac{\partial \mathbf{f}^{\mathrm{T}}\left(\Theta_{2}\right)}{\partial \Theta_{2}} \mathbf{G}_{\mathrm{c}}\left(\Theta_{1}\right) \mathbf{c}^{\mathrm{in}}\left(\lambda_{\mathrm{m}}\right) & \cdots \\
\vdots & \vdots & \ddots
\end{array}\right)\right|_{\lambda_{\mathrm{m}, \infty}}
$$

und dem Eingangsvektor:

$$
\mathbf{B}_{1}=\left.\left(\begin{array}{c}
\mathbf{f}^{\mathrm{T}}\left(\Theta_{1}\right) \frac{\partial \mathbf{c}^{\mathrm{in}}\left(\lambda_{\mathrm{m}}\right)}{\partial \lambda_{\mathrm{m}}} \\
\mathbf{f}^{\mathrm{T}}\left(\Theta_{2}\right) \mathbf{G}_{\mathrm{c}}\left(\Theta_{1}\right) \frac{\partial \mathrm{c}^{\mathrm{in}}\left(\lambda_{\mathrm{m}}\right)}{\partial \lambda_{\mathrm{m}}} \\
\vdots
\end{array}\right)\right|_{\substack{\lambda_{\mathrm{m}, \infty} \\
\boldsymbol{\Theta}_{\infty}}}
$$

\footnotetext{
${ }^{1}$ An dieser Stelle wird darauf hingewiesen, dass bei der Erstellung des Emissionsmodells auf seine Differenzierbarkeit geachtet werden muss.
} 


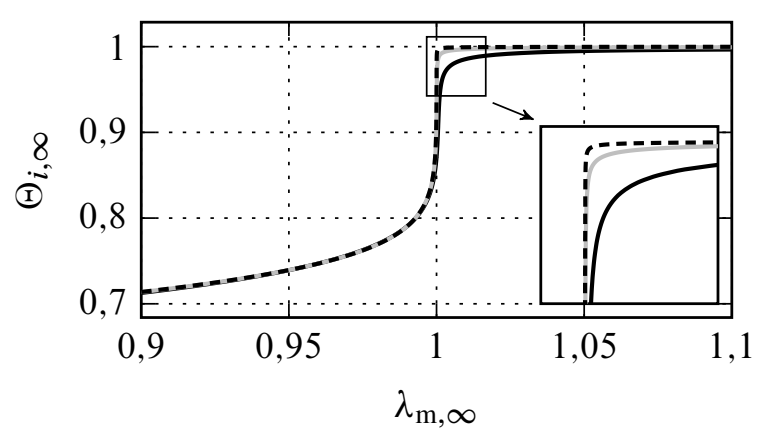

Bild 4.4: Verlauf der stationären Sauerstofffüllstände: 1. Modellzelle (-), 2. Modellzelle ( -$), 3$. Modellzelle (----)

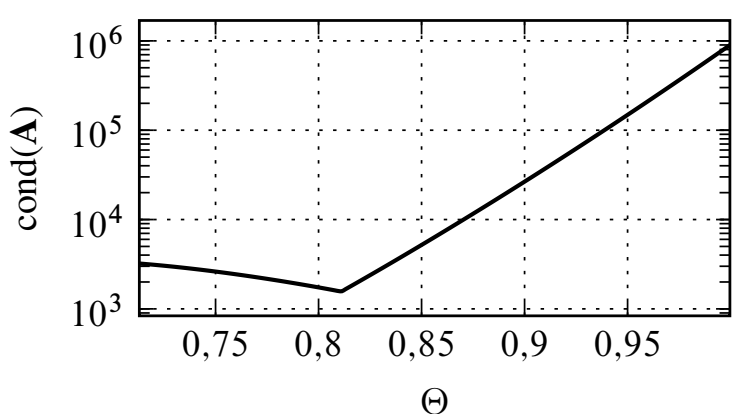

Bild 4.5: Verlauf der Konditionszahl der Matrix A in Gleichung (3.26)

Da zwischen den Elementen der Matrix $\mathbf{G}_{\mathrm{c}}$ und den Gewichtsfunktionen ein affiner Zusammenhang besteht, siehe Abschnitt 3.2, kann die Ableitung von $\mathbf{G}_{\mathrm{c}}$ leicht durch die abgeleiteten Gewichtsfunktionen dargestellt werden. Aufgrund der Reihenschaltung der Zellen und der Abhängigkeit jeder Zelle nur von den Zellen davor, ergibt sich für $\mathbf{A}_{1}$ in Gleichung (4.10) eine untere Dreiecksmatrix. Die analytische Linearisierung des nichtlinearen Katalysatormodells, die wichtig für seinen praktischen Einsatz ist und in der einschlägigen Literatur bisher nicht betrachtet wird, wird erst durch die neuartige Modelldarstellung mittels Gewichtsfunktionen ermöglicht.

Stationäre Arbeitspunkte Für die Linearisierung wird der stationäre Arbeitspunkt $\Theta_{\infty}$ benötigt, der sich für einen stationären Lambdawert $\lambda_{\mathrm{m}, \infty}$ einstellt. Der stationäre Arbeitspunkt berechnet sich leicht anhand des Zustandsraummodells. Dafür wird die linke Seite der Vektordifferentialgleichung (3.39) zu Null gesetzt

$$
\left(\begin{array}{c}
0 \\
0 \\
\vdots \\
0
\end{array}\right)=\left(\begin{array}{c}
\mathbf{f}^{\mathrm{T}}\left(\Theta_{1, \infty}\right) \mathbf{c}^{\mathrm{in}}\left(\lambda_{\mathrm{m}, \infty}\right) \\
\mathbf{f}^{\mathrm{T}}\left(\Theta_{2, \infty}\right) \mathbf{G}_{\mathrm{c}}\left(\Theta_{1, \infty}\right) \mathbf{c}^{\mathrm{in}}\left(\lambda_{\mathrm{m}, \infty}\right) \\
\vdots \\
\mathbf{f}^{\mathrm{T}}\left(\Theta_{n_{\mathrm{c}}, \infty}\right) \mathbf{G}_{\mathrm{c}}\left(\Theta_{n_{\mathrm{c}}-1, \infty}\right) \cdots \mathbf{G}_{\mathrm{c}}\left(\Theta_{1, \infty}\right) \mathbf{c}^{\mathrm{in}}\left(\lambda_{\mathrm{m}, \infty}\right)
\end{array}\right)
$$

und die erste Gleichung der Matrizengleichung (4.12) nach dem Sauerstofffüllstand $\Theta_{1, \infty}$ aufgelöst. Anschließend kann der Wert für $\Theta_{1, \infty}$ in die zweite Gleichung der Matrizengleichung eingesetzt und diese nach $\Theta_{2, \infty}$ aufgelöst werden. Auf diese Weise werden alle Sauerstofffüllstände am Arbeitspunkt schrittweise bestimmt. Diese Vorgehensweise stützt sich darauf, dass jede Zelle nur von den Zellen davor abhängt. Bild 4.4 zeigt die stationären Sauerstofffüllstände in Abhängigkeit des Lambdawertes $\lambda_{m, \infty}$. Wie in Abschnitt 4.1 erläutert, kann nicht die gesamte Menge an $\mathrm{Ce}_{2} \mathrm{O}_{4}$ bis auf $\mathrm{Ce}_{2} \mathrm{O}_{3}$ reduziert werden, sodass sich der Sauerstofffüllstand immer im Bereich von etwa 0,7 bis 1 bewegt. Der steile Anstieg der Kurven in Bild 4.4 deutet auf die hohe Empfindlichkeit des Sauerstofffüllstandes auf die Eingangskonzentrationen und damit auf den Lambdawert $\lambda_{\mathrm{m}}$ hin. Für Lambdawerte größer Eins ist der Sauerstoffspeicher nahezu gefüllt und ändert sich nur wenig mit weiter ansteigendem Lambdawert. Diese Beobachtungen legen die Vermutung nahe, dass das Katalysatormodell sehr sensitiv bezüglich Parameterunsicherheiten und numerischen Fehlern bei der 
a)

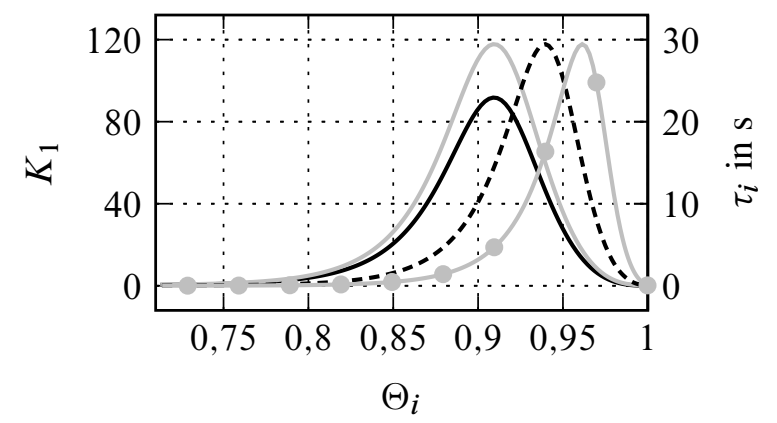

b)

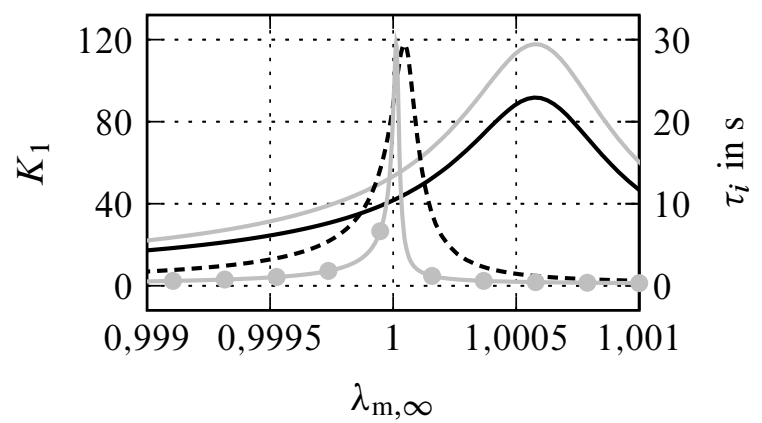

Bild 4.6: Zeitkonstanten des linearisierten Modells und stationäre Verstärkung der ersten Modellzelle in Abhängigkeit a) des Sauerstofffüllstandes und b) des Lambdawertes innerhalb des Lambdafensters: $K_{1}(-), \tau_{1}(-), \tau_{2} / 4.8(---), \tau_{3} / 16.4(--)$

Simulation reagieren wird. Tatsächlich ist das Gleichungssystem (3.26) schlecht konditioniert. In Bild 4.5 ist der Verlauf der Konditionszahl der Matrix A in Abhängigkeit des Sauerstofffüllstands der Modellzelle dargestellt. Mit der Konditionszahl lassen sich die Auswirkungen von kleinen Änderungen der Matrix A und des Vektors Bc ${ }^{\text {in }}$ auf die Lösung des Gleichungssystems (3.26) abschätzen [108]. Das Minimum der Konditionszahl wird bei einem Sauerstofffüllstand von etwa 0,81 erreicht. Ab diesem Punkt steigt die Konditionszahl in etwa exponentiell an, was in etwa mit dem Anfang des Bereichs der hohen Steigungen in Bild 4.4 korreliert. Für Lambdawerte größer Eins bleibt das Gleichungssystem schlecht konditioniert, weil sich die Sauerstofffüllstände mit weiter steigendem Lambdawert nur geringfügig ändern.

Die Kurven der hinteren Zellen in Bild 4.4 verlaufen steiler als die der vorderen Zellen. Dies erhöht die Schwierigkeit, bei einer Diskretisierung des Katalysatormodells in viele Zellen den Lambdawert $\lambda_{\mathrm{m}}$ zu finden, der zu einem bestimmten Sauerstofffüllstand der letzten Zelle führt. Dieser numerische Aspekt ist ein weiterer Grund für die Verwendung eines Katalysatormodells mit nur drei Zellen.

Zeitkonstanten Die im vorherigen Abschnitt getroffenen Aussagen zur Systemdynamik können nach der Linearisierung anhand einer Betrachtung der Zeitkonstanten validiert werden. In Bild 4.6a sind die Zeitkonstanten des linearisierten Modells in Abhängigkeit des Sauerstofffüllstandes der jeweiligen Modellzelle dargestellt. Die Zeitkonstanten ändern sich beträchtlich mit dem Sauerstofffüllstand und belegen das stark nichtlineare Verhalten des Katalysators. Die Zeitkonstanten der zweiten und der dritten Modellzelle sind der Übersichtlichkeit halber skaliert. Die skalierten Verläufe haben eine ähnliche Form, wobei das Maximum der Zeitkonstanten der hinteren Modellzellen bei immer höheren Sauerstofffüllständen angenommen wird. Dies hängt damit zusammen, dass sich die Gaskonzentrationen entlang des Katalysators verändern und damit jede Modellzelle andere Eingangskonzentrationen erfährt. In Bild 4.6a ist zusätzlich noch die stationäre Verstärkung der ersten Zelle des Modells dargestellt, die einen ähnlichen Verlauf wie die entsprechende Zeitkonstante aufweist. In Bild 4.6b sind die Zeitkonstanten und die stationäre Verstärkung in Abhängigkeit des Lambdawertes aufgetragen. Die Zeitkonstanten der hinteren Modellzellen 
weisen einen schmaleren Verlauf auf, was mit dem steileren Anstieg der Kurven in Bild 4.4 zusammenhängt. An Bild 4.6b ist das stark nichtlineare Verhalten des Katalysators im Bereich des Lambdafensters sehr gut nachzuvollziehen.

\subsection{Berechnung von Strukturmaßen}

Keine systemtheoretische Analyse wäre ohne die Betrachtung von Eigenschaften wie der Steuerbarkeit und der Beobachtbarkeit vollständig. Für die Analyse in diesem Abschnitt wird als Ausgang des Zustandsraummodells (3.39) der Lambdawert gewählt [4]:

$$
\begin{aligned}
& y=\frac{2 c_{\mathrm{CO}_{2}}+c_{\mathrm{H}_{2} \mathrm{O}}+2 c_{\mathrm{O}_{2}}+c_{\mathrm{CO}}}{2 c_{\mathrm{CO}_{2}}+c_{\mathrm{H}_{2} \mathrm{O}}+2 c_{\mathrm{CO}}+c_{\mathrm{H}_{2}}} \\
& =\frac{\left(\begin{array}{lllll}
2 & 1 & 2 & 1 & 0
\end{array}\right) \mathbf{G}_{\mathrm{c}}\left(\Theta_{3}\right) \mathbf{G}_{\mathrm{c}}\left(\Theta_{2}\right) \mathbf{G}_{\mathrm{c}}\left(\Theta_{1}\right) \mathbf{c}^{\mathrm{in}}\left(\lambda_{\mathrm{m}}\right)}{\left(\begin{array}{lllll}
2 & 1 & 0 & 2 & 1
\end{array}\right) \mathbf{G}_{\mathrm{c}}\left(\Theta_{3}\right) \mathbf{G}_{\mathrm{c}}\left(\Theta_{2}\right) \mathbf{G}_{\mathrm{c}}\left(\Theta_{1}\right) \mathbf{c}^{\mathrm{in}}\left(\lambda_{\mathrm{m}}\right)} \text {. }
\end{aligned}
$$

Damit ist die Ausgangsfunktion sehr komplex und erschwert die Untersuchungen direkt am nichtlinearen Modell. Zum Beispiel wird nach [2] für die Überprüfung der Beobachtbarkeit direkt am nichtlinearen System die Abbildung

$$
\mathbf{z}=\left(\begin{array}{c}
y \\
\dot{y} \\
\ddot{y}
\end{array}\right)=\mathbf{q}\left(\boldsymbol{\Theta}, \lambda_{\mathrm{m}}, \dot{\lambda}_{\mathrm{m}}, \ddot{\lambda}_{\mathrm{m}}\right)
$$

durch die Bildung der zeitlichen Ableitungen des Ausgangs $y$ aufgestellt. Ein hinreichendes Kriterium für die globale Beobachtbarkeit des Systems ist die eindeutige Auflösung der Abbildung q nach $\Theta$, was für das Katalysatormodell mit drei Zellen schwierig zu überprüfen ist. Die einfachere Untersuchung der lokalen Beobachtbarkeit anhand des Rangs der Matrix

$$
\mathbf{M}_{\mathrm{b}}=\frac{\partial \mathbf{q}\left(\boldsymbol{\Theta}, \lambda_{\mathrm{m}}, \dot{\lambda}_{\mathrm{m}}, \ddot{\lambda}_{\mathrm{m}}\right)}{\partial \boldsymbol{\Theta}}
$$

erweist sich ebenfalls als schwierig, da die symbolische Berechnung der Matrix $\mathbf{M}_{\mathrm{b}}$ und die Generierung einer ausführbaren Funktion daraus mit einem hohen Rechenaufwand verbunden ist. Aus diesem Grund wird in diesem Abschnitt vom linearisierten Modell ausgegangen und dementsprechend lineare Strukturmaße untersucht.

Zur Untersuchung der Steuerbarkeit und der Beobachtbarkeit können die klassischen Kriterien von Kalman, Gilbert oder Hautus angewendet werden, siehe [60]. Diese Kriterien liefern das vollständige Vorhandensein dieser Systemeigenschaften. Jedoch berichten z. B. Balenovic u. a. [7], Fiengo u. a. [30] und Muske und Peyton Jones [69] darüber, dass der Katalysator in Abhängigkeit des Sauerstofffüllstandes unterschiedlich gut beobachtbar ist. Um die Intensität der Systemeigenschaften bestimmen zu können, werden in diesem Abschnitt Strukturmaße berechnet. Diese umfassen das Steuerbarkeits-, das Beobachtbarkeits- und das Dominanzmaß, welches den Einfluss der Ein- und der Ausgangsgrößen auf das Systemverhalten gleichzeitig erfasst. Daher ist das Dominanzmaß primär bei der Ordnungsreduktion von Interesse, siehe die Arbeit von Litz [55]. In [82] ist die Herleitung und ein Vergleich der im Folgenden vorgestellten Strukturmaße zu finden. 
Transformation auf Modalform Der Ausgangspunkt der Berechnung der Strukturmaße ist die Transformation des Systems auf Modalform. Für jeden stationären Arbeitspunkt, bis auf die wenigen Schnittpunkte in Bild 4.6b, sind die Eigenwerte paarweise verschieden, sodass eine Transformation auf die Modalkoordinaten $\mathbf{z}$ möglich ist:

$$
\Delta \Theta=\mathbf{Q z} .
$$

Die Modalmatrix $\mathbf{V}$ in Gleichung (4.16) setzt sich aus den $n_{\mathrm{c}}$ linear unabhängigen Rechtseigenvektoren $v_{k}$ der Systemmatrix $\mathbf{A}_{1}$ zusammen:

$$
\mathbf{V}=\left(\begin{array}{lll}
\mathbf{v}_{1} & \cdots & \mathbf{v}_{n_{\mathrm{c}}}
\end{array}\right) .
$$

Hierbei bezeichnet $k$ den Zellenindex. Die Inverse $\mathbf{W}$ von $\mathbf{V}$ setzt sich aus den entsprechenden Linkseigenvektoren zusammen:

$$
\mathbf{V}^{-1}=\mathbf{W}=\left(\begin{array}{c}
\mathbf{w}_{1}^{\mathrm{T}} \\
\vdots \\
\mathbf{w}_{n_{\mathrm{c}}}^{\mathrm{T}}
\end{array}\right)
$$

Anwendung der Transformation (4.16) auf die linearisierte Vektordifferentialgleichung (4.9) und auf die linearisierte Ausgangsgleichung (4.13) liefert schließlich die Modalform

$$
\begin{aligned}
\frac{\mathrm{d}}{\mathrm{d} t} \mathbf{z} & =\hat{\mathbf{A}} \mathbf{z}+\hat{\mathbf{B}} \Delta \lambda_{\mathrm{m}}, \\
\Delta y & =\hat{\mathbf{C}} \mathbf{z}
\end{aligned}
$$

mit den Matrizen [55, 82]:

$$
\begin{aligned}
& \hat{\mathbf{A}}=\mathbf{W A}_{1} \mathbf{V}=\left(\begin{array}{ccc}
a_{1} & & \mathbf{0} \\
& \ddots & \\
\mathbf{0} & & a_{n_{\mathrm{c}}}
\end{array}\right) \text {, } \\
& \hat{\mathbf{B}}=\mathbf{W B}_{1}=\left(\begin{array}{c}
\hat{\mathbf{b}}_{1}^{\mathrm{T}} \\
\vdots \\
\hat{\mathbf{b}}_{n_{\mathrm{c}}}^{\mathrm{T}}
\end{array}\right), \\
& \hat{\mathbf{C}}=\mathbf{C}_{\mathrm{l}} \mathbf{V}=\left(\begin{array}{lll}
\hat{\mathbf{c}}_{1} & \cdots & \hat{\mathbf{c}}_{n_{\mathrm{c}}}
\end{array}\right) .
\end{aligned}
$$

In Gleichung (4.20) bezeichnen $a_{1}$ bis $a_{n_{\mathrm{c}}}$ die Eigenwerte der Systemmatrix $\mathbf{A}_{1}$. In Gleichung (4.22) steht $\mathbf{C}_{1}$ für die Ausgangsmatrix des linearisierten Zustandsraummodells, welche sich durch die Ableitung von Gleichung (4.13) nach den einzelnen Sauerstofffüllständen ergibt.

Steuer- und Beobachtbarkeit Nach Lückel/Müller berechnet sich das Steuerbarkeitsmaß $\kappa_{\mathrm{B}}$ nach der Vorschrift

$$
\kappa_{\mathrm{B}, k}=\frac{\overline{\mathbf{w}}_{k}^{\mathrm{T}} \mathbf{B}_{1} \mathbf{B}_{1}^{\mathrm{T}} \mathbf{w}_{k}}{\overline{\mathbf{w}}_{k}^{\mathrm{T}} \mathbf{w}_{k}} \mathrm{e}^{-2 \operatorname{Re}\left(a_{k}\right)}
$$

und das Beobachtbarkeitsmaß $\kappa_{\mathrm{C}}$ nach der Vorschrift:

$$
\kappa_{\mathrm{C}, k}=\frac{\overline{\mathbf{v}}_{k}^{\mathrm{T}} \mathbf{C}_{l}^{\mathrm{T}} \mathbf{C}_{l} \mathbf{v}_{k}}{\overline{\mathbf{v}}_{k}^{\mathrm{T}} \mathbf{v}_{k}} \mathrm{e}^{-2 \operatorname{Re}\left(a_{k}\right)} .
$$


Hierbei bezeichnen $\overline{\mathbf{w}}_{k}$ und $\overline{\mathbf{v}}_{k}$ die entsprechenden konjugiert komplexen Eigenvektoren von $\mathbf{A}_{1}$. Der Faktor $\mathrm{e}^{-2 \operatorname{Re}\left(a_{k}\right)}$ koppelt die Steuerbarkeit und die Beobachtbarkeit mit der Systemstabilität und kann in bestimmten Fällen zu ungewollten, starken Verzerrungen der Strukturmaße führen. Aus diesem Grund und in Anlehnung an Litz [55] werden im Folgenden die von der Kopplung zum Eigenwert bereinigten Strukturmaße berechnet.

Dominanzmaß Die von Litz entwickelten Dominanzmaße ordnen jedem Systemeigenwert einen Zahlenwert zu, welcher den Beitrag des Eigenwertes zum Übertragungsverhalten des Systems widerspiegelt $[55,82]$. Hohe Dominanzmaße deuten auf wichtige Systemanteile hin, die bei einer Ordnungsreduktion unbedingt berücksichtigt werden müssen und umgekehrt. Das Steuerbarkeitsmaß $\kappa_{\mathrm{B}, k}$ berücksichtigt die Eingangsverstärkungen des Systems und das Beobachtbarkeitsmaß $\kappa_{\mathrm{C}, k}$ die entsprechenden Ausgangsverstärkungen. Die Dominanzmaße berücksichtigen beides und zusätzlich die stationären Verstärkungen im $k$-ten Übertragungspfad. Es kann gezeigt werden, dass durch geringfügige Änderungen der hier gezeigten Definitionen, die Dominanzmaße das Produkt des Steuerbarkeits- und des Beobachtbarkeitsmaßes sind [55]. Da das Katalysatormodell ein Eingrößensystem darstellt, sind das Summen- $S_{k}$ und das Maximalmaß $M_{k}$ gleich. Sie berechnen sich nach der Vorschrift:

$$
S_{k}=M_{k}=\left|\frac{\hat{c}_{k} \hat{b}_{k}}{a_{k}}\right| \text {. }
$$

Konsistenz der Strukturmaße Bevor die Strukturmaße für das Katalysatormodell berechnet werden, wird noch ein Nachteil der bisher vorgestellten Strukturmaße angesprochen, welcher die mangelnde Konsistenz zu den Kalman'schen Begriffen der Steuerbarkeit und der Beobachtbarkeit betrifft. Unter Konsistenz ist zu verstehen, dass ein Strukturmaß den Verlust der Steuerbarkeit und der Beobachtbarkeit in jedem Fall richtig wiedergibt. Nach den Überlegungen von Litz muss ein hohes Strukturmaß nicht unbedingt mit einer guten Steuerbarkeit, Beobachtbarkeit oder hohen Dominanz verknüpft sein. Dies kann insbesondere dann der Fall sein, wenn die Systemeigenwerte nahe beieinander liegen. Als Faustregel gilt, dass sich reellwertige Eigenwerte um mindestens $15 \%$ voneinander unterscheiden sollten, um nicht als benachbart zu gelten. Für niedrige Strukturmaße gilt dies jedoch nicht. Kleine Strukturmaße sind in jedem Fall hinreichend für schlechte Steuerbarkeit, Beobachtbarkeit oder niedrige Dominanz. Beim Katalysatormodell unterscheiden sich die Eigenwerte nur im Bereich des Lambdafensters stark voneinander. Deshalb werden im Folgenden die Aussagen der Strukturmaße mit den Ergebnissen der bisher gemachten Systemanalyse auf Plausibilität überprüft.

Berechnung der Strukturmaße für das Katalysatormodell Die starke Änderung der Systemdynamik im Bereich des Lambdafensters bereitet Schwierigkeiten bei der numerischen Berechnung der Strukturmaße, insbesondere bei der Berechnung des Dominanzmaßes. Daher werden bei der Diskretisierung des Arbeitsbereichs weniger Stützstellen im Bereich des Lambdafensters als bisher gewählt. 
a)

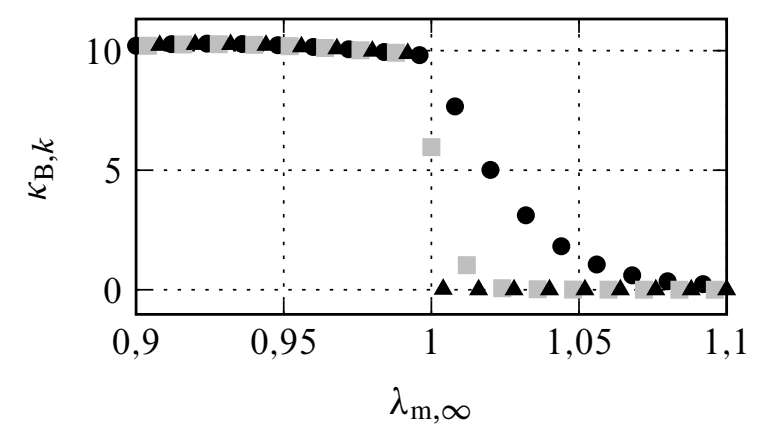

b)

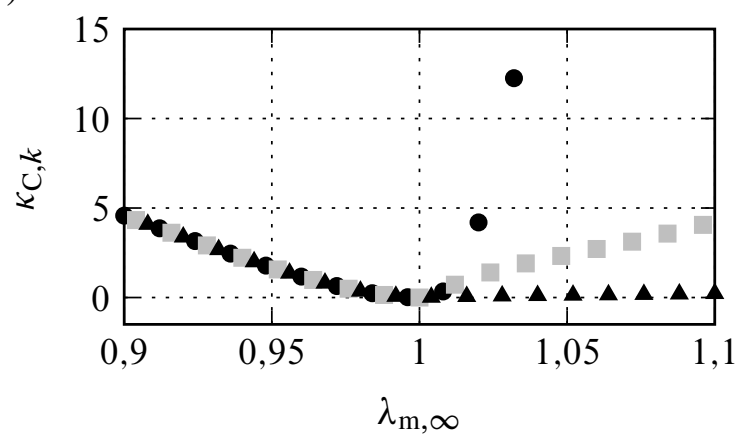

Bild 4.7: Verlauf a) des Steuerbarkeits- und b) des Beobachtbarkeitsmaßes nach Lückel/Müller für ein Katalysatormodell mit drei Zellen: 1. Modellzelle (• ), 2. Modellzelle ( $\square$ ), 3. Modellzelle ( $\Delta$ )

In Bild 4.7a ist das Steuerbarkeitsmaß in Abhängigkeit des Lambdawertes $\lambda_{\mathrm{m}, \infty}$ für das in drei Zellen diskretisierte Katalysatormodell dargestellt. Für Lambdawerte von 0,9 bis 1 ist das Steuerbarkeitsmaß der drei Modellzellen etwa gleich und nimmt mit steigendem Lambdawert ab. Im Bereich des Lambdafensters sinkt das Maß vor allem der hinteren Modellzellen schnell auf nahezu Null, während das Maß der ersten Modellzelle langsam mit weiter steigendem Lambdawert abnimmt. Betrachtet man den Verlauf der stationären Arbeitspunkte in Bild 4.6a, so ist dieses Verhalten plausibel. Denn für Lambdawerte von 0,9 bis 1 bewirkt eine moderate Änderung des Lambdawertes am Katalysatoreingang auch eine moderate Änderung der Sauerstofffüllstände, d. h. die Zustände sind gut steuerbar. Mit weiter steigendem Lambdawert wird der Sauerstoffspeicher im Bereich des Lambdafensters schnell gefüllt und hängt anschließend nur wenig vom Lambdawert $\mathrm{ab}$, d. h. die Zustände sind schlecht steuerbar. Im Lambdabereich von 1 bis 1,1 hängt der Sauerstofffüllstand der ersten Modellzelle am stärksten vom Lambdawert ab, was die höheren Werte des Maßes erklärt.

In Bild 4.7b ist der Verlauf des Beobachtbarkeitsmaßes dargestellt. Wieder verläuft das Maß der drei Modellzellen für Lambdawerte von 0,9 bis 1 etwa gleich und nimmt mit steigendem Lambdawert ab. Im Bereich des Lambdafensters sinkt das Maß aller Modellzellen auf nahezu Null und bestätigt damit die schlechte Beobachtbarkeit im für den geregelten Betrieb relevanten Bereich. Mit weiter steigendem Lambdawert steigt insbesondere das Maß der ersten Modellzelle, während das Maß der dritten Modellzelle bei nahezu Null verbleibt. Der Verlust der Beobachbarkeit im Bereich des Lambdafensters ist zum einen aus der Literatur bekannt und zum anderen einleuchtend. Denn das Ziel des Katalysators ist es, die Lambdaabweichungen am Katalysatoreingang zu dämpfen und am Katalysatorausgang möglichst stöchiometrische Bedingungen zu erzielen. Aus dem stöchiometrischen Lambdawert hinter dem Katalysator kann jedoch nicht auf den genauen Sauerstofffüllstand geschlossen werden, d.h. die schlechte Beobachtbarkeit ist aus Sicht der Schadstoffkonvertierung sogar erwünscht. Für stationäre, nicht stöchiometrische Bedingungen am Katalysatoreingang kann der Sauerstofffüllstand ermittelt werden, siehe Bild 4.4, und daher steigt das Maß außerhalb des Lambdafensters. Betrachtet man die Ausgangskonzentrationen in Abhän- 

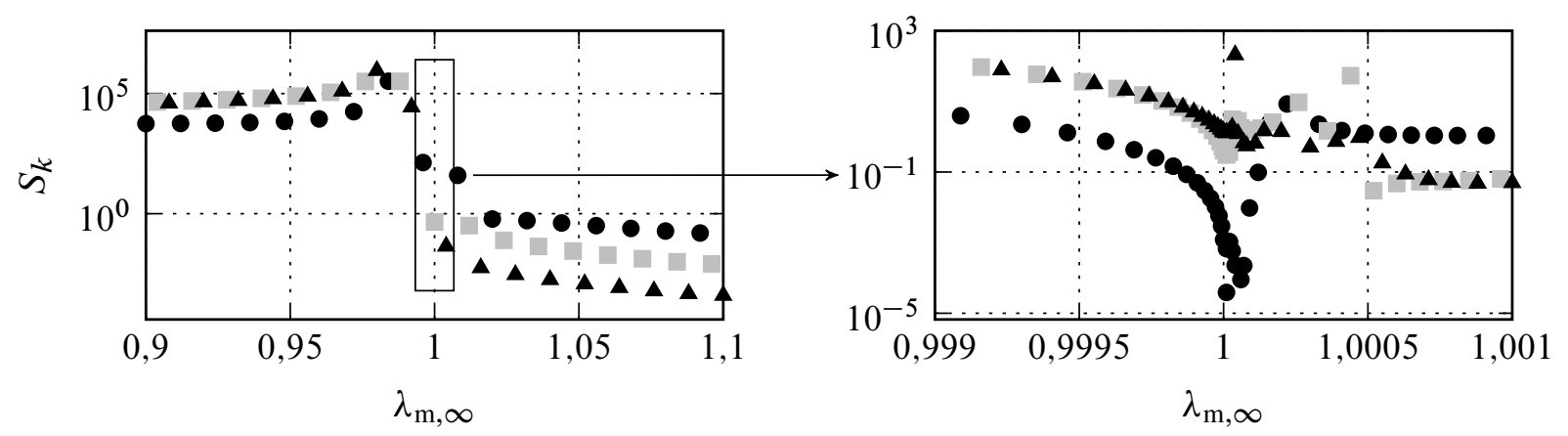

Bild 4.8: Verlauf des Dominanzmaßes nach Litz für ein Katalysatormodell mit drei Zellen: 1.

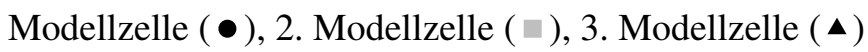

gigkeit aller Sauerstofffüllstände

$$
\mathbf{c}=\mathbf{G}_{\mathrm{c}}\left(\Theta_{3}\right) \mathbf{G}_{\mathrm{c}}\left(\Theta_{2}\right) \mathbf{G}_{\mathrm{c}}\left(\Theta_{1}\right) \mathbf{c}^{\mathrm{in}}\left(\lambda_{\mathrm{m}}\right)
$$

so ist weiterhin plausibel, dass die erste Modellzelle, deren Sauerstofffüllstand sich am stärksten für Lambdawerte größer Eins ändert, auch am stärksten die Ausgangskonzentrationen beeinflusst und daher am besten beobachtbar ist.

Das Dominanzmaß ist in Bild 4.8 dargestellt. Man sieht, dass es die Eigenschaften des Steuerbarkeits- und des Beobachtbarkeitsmaßes vereint, wenn von den hohen Werten bei $\Theta=0,98$ abgesehen wird. Für Lambdawerte $<1$ sind alle drei Modellzellen dominant und das Maß nimmt im Bereich des Lambdafensters sehr stark ab. Die höhere Dominanz der hinteren Modellzellen im Bereich des Lambdafensters liegt dabei an ihren größeren Zeitkonstanten, siehe Bild 4.6b. Für Lambdawerte $>1$ hat die erste Modellzelle eine höhere Dominanz, was mit ihrer besseren Steuerbarkeit und Beobachtbarkeit in diesem Bereich zusammenhängt. Am Verlauf des Dominanzmaßes ist auch zu sehen, dass eine weitere Ordnungsreduktion, d. h. eine Diskretisierung des Modells in weniger als drei Zellen, nicht sinnvoll ist, da alle drei Modellzellen ähnlich stark am dynamischen Verhalten beteiligt sind.

Weiterführende Überlegungen zu den Strukturmaßen Das Problem der Konsistenz der Strukturmaße von Lückel/Müller und Litz hat zur Entwicklung des konsistenten Steuerbarkeitsmaßes von Benninger und Rivoir [10] geführt. Das Maß beruht auf einer Betrachtung der Steuerenergie, welche zur gezielten Veränderung des Systemzustandes erforderlich ist. Die Berechnung dieses Maßes für den Katalysator ist aufwendiger als die in diesem Abschnitt vorgestellten Maße, stellt jedoch einen interessanten Aspekt dar, welcher in weiterführenden Arbeiten untersucht werden kann.

Die klassischen Ja-Nein Aussagen des Kalman-, des Gilbert- oder des Hautus-Kriteriums basieren auf der Überprüfung der Regularität einer Matrix. Ein Maß für die Regularität einer Matrix A ist die in Abschnitt 4.3 verwendete Konditionszahl $\kappa(\mathbf{A})$. Eine gut konditionierte Matrix hat eine Kondition nahe Eins, während eine Matrix mit einer Kondition $\kappa(\mathbf{A}) \gg 1$ schlecht konditioniert und für $\kappa(\mathbf{A}) \rightarrow \infty$ singulär ist. Es ist naheliegend, die Kondition der Steuerbarkeits- oder der 
a)

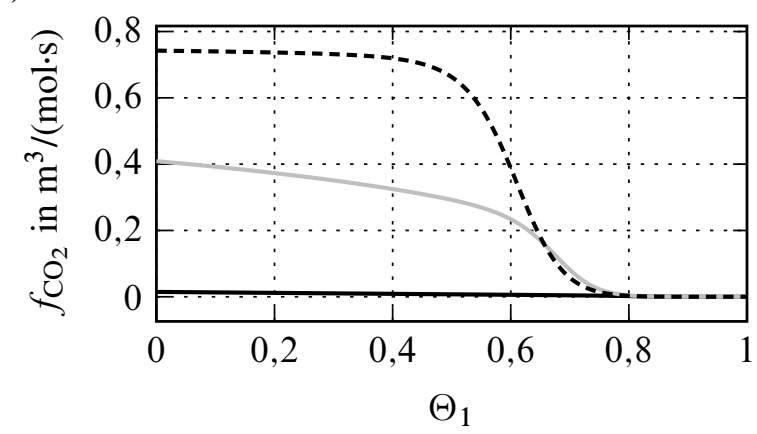

b)

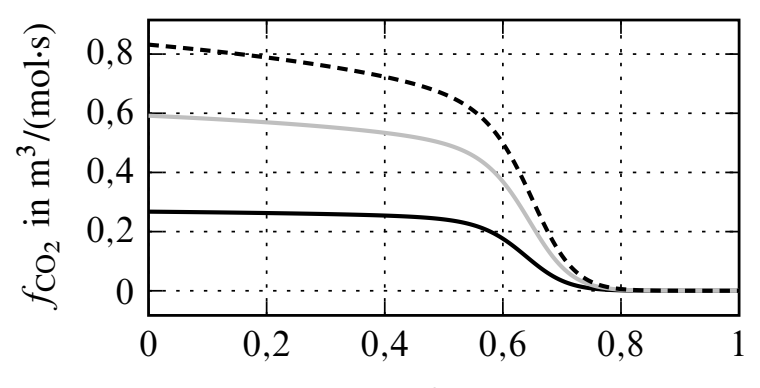

$\Theta_{1}$

Bild 4.9: Verlauf der Gewichtsfunktion von $\mathrm{CO}_{2}$ : a) bei einer Raumgeschwindigkeit von $24600 \mathrm{~h}^{-1}$ für die Temperaturen $700 \mathrm{~K}(-), 810 \mathrm{~K}(-)$ und $920 \mathrm{~K}(----)$; b) bei einer Temperatur von $810 \mathrm{~K}$ für die Raumgeschwindigkeiten $16900 \mathrm{~h}^{-1}(-), 43800 \mathrm{~h}^{-1}(-)$ und $70800 \mathrm{~h}^{-1}(----)$

Beobachtbarkeitsmatrix als ein Maß für die Intensität dieser Eigenschaften zu nutzen. Definiert man die Kondition über die Spektralnorm, so ist diese das Verhältnis des größten zum kleinsten Singulärwert:

$$
\kappa(\mathbf{A})=\frac{\sigma_{\max }(\mathbf{A})}{\sigma_{\min }(\mathbf{A})} .
$$

Betrachtet man nun eine beliebige Funktion, die den Wertebereich der Konditionszahl auf den Bereich von Eins bis Null abbildet, so ist dieses Maß konsistent zum Kriterium von Kalman. Ein ähnlicher Ansatz wird in [54] gemacht und ein Beobachtbarkeitsmaß auf Basis des invertierten kleinsten Singulärwerts der Matrix in Gleichung (4.15) definiert.

\subsection{Einfluss der Motorarbeitspunktparameter auf die Systemeigenschaften}

In diesem Abschnitt wird der Einfluss der Temperatur und des Abgasmassenstroms auf die in den letzten zwei Abschnitten diskutierten Systemeigenschaften untersucht. Der Einfachheit halber werden die Temperaturen $T_{\mathrm{g}}^{\mathrm{in}}, T_{\mathrm{g}}$ und $T_{\mathrm{s}}$ gleichgesetzt. Für die Untersuchungen werden zwei Testszenarien erstellt: Im ersten Testszenario wird die Temperatur variiert und der Abgasmassenstrom konstant gehalten und im Zweiten wird bei einer konstanten Temperatur der Abgasmassenstrom bzw. die Raumgeschwindigkeit variiert. Die minimalen Werte entsprechen denen am ersten Motorarbeitspunkt und die maximalen Werte denen am dritten Motorarbeitspunkt, siehe Anhang A.1.

Gewichtsfunktionen In Bild 4.9 ist der Übersichtlichkeit halber nur die Gewichtsfunktion von $\mathrm{CO}_{2}$ dargestellt, wobei sich die restlichen vier Gewichtsfunktionen qualitativ ähnlich verhalten. Bei einer Temperatur von $700 \mathrm{~K}$ läuft die Reaktion (R1), in der $\mathrm{CO}_{2}$ als Reaktant vorkommt, sehr langsam ab, sodass die entsprechende Gewichtsfunktion sehr nahe bei Null liegt. Mit steigender 
a)

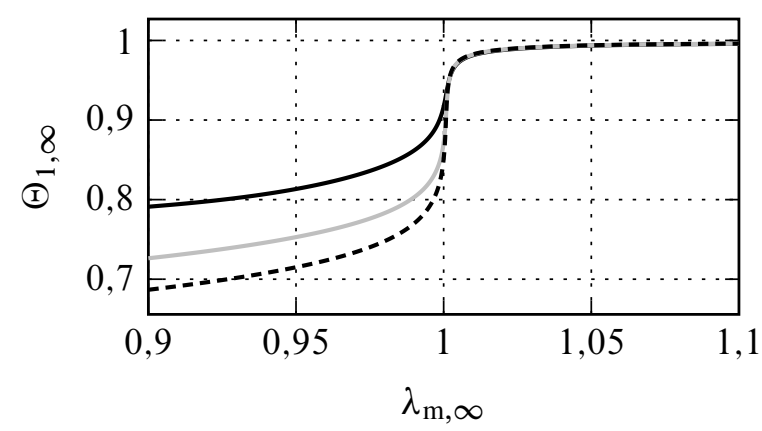

b)

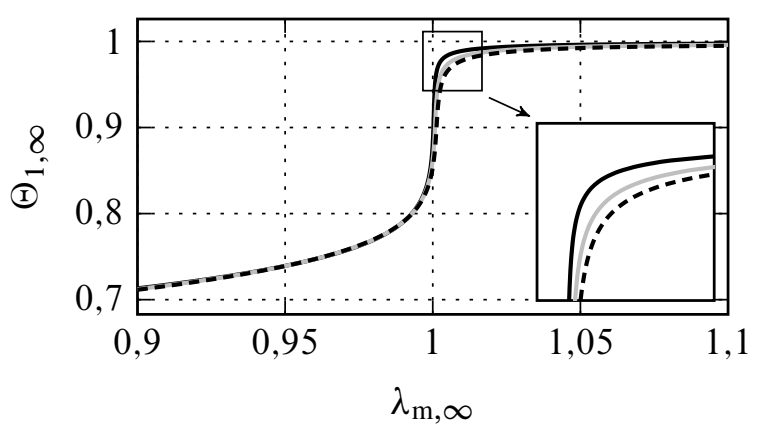

Bild 4.10: Verlauf des stationären Arbeitspunkts der ersten Zelle des Modells: a) bei einer Raumgeschwindigkeit von $24600 \mathrm{~h}^{-1}$ für die Temperaturen $700 \mathrm{~K}(-), 810 \mathrm{~K}(-)$ und $920 \mathrm{~K}$ (---); b) bei einer Temperatur von $810 \mathrm{~K}$ für die Raumgeschwindigkeiten $16900 \mathrm{~h}^{-1}$ $(-), 43800 \mathrm{~h}^{-1}(-)$ und $70800 \mathrm{~h}^{-1}(----)$

Temperatur steigen die Geschwindigkeitskonstanten der Reaktionen und die Werte der Gewichtsfunktionen nehmen entsprechend zu, d. h. die chemischen Spezies werden reaktionsfreudiger. Ein ähnliches Verhalten ist auch bei der Variation des Abgasmassenstroms in Bild 4.9b zu sehen, wobei die Gewichtsfunktion eher gestreckt wird, als dass sie ihre grundsätzliche Form ändert. Werden die Gleichungen (3.20) und (3.21) addiert

$$
\frac{\mathrm{R} \dot{m}}{A_{\mathrm{cs}} p_{\mathrm{exh}} \bar{M} \Delta z}\left(c_{i} T_{\mathrm{g}}-c_{i}^{\mathrm{in}} T_{\mathrm{g}}^{\mathrm{in}}\right)=A_{\text {cat }} L \dot{s}_{i}
$$

und die Beträge auf beiden Seiten von Gleichung (4.28) betrachtet, so ist ersichtlich, dass eine Erhöhung der Temperatur oder des Abgasmassenstroms auch eine Erhöhung des Reaktionsquellterms $\dot{s}_{i}$ erfordert. Die Reaktionsquellterme werden durch die Gewichtsfunktionen dargestellt, siehe Gleichung (3.35), sodass ihre Beträge bzw. Differenzen untereinander ebenfalls steigen müssen.

Stationäre Arbeitspunkte In Bild 4.10 ist der Verlauf des stationären Arbeitspunkts der ersten Zelle des Modells abgebildet. Eine Erhöhung der Temperatur führt dazu, dass das Ceroxid stärker reduziert werden kann, was durch die gesteigerte Reaktionsfreudigkeit bedingt ist. Interessant ist weiterhin, dass die Kurven in Bild 4.10a unabhängig von der Temperatur ab einem Sauerstofffüllstand von etwa 0,9 gleich verlaufen. Die Variation des Abgasmassenstroms verändert die Verläufe in Bild 4.10b nur geringfügig.

Zeitkonstanten Die bisher gemachte Beobachtung, dass das System erst bei höheren Temperaturen aktiviert wird, ist auch an der Zeitkonstante der ersten Zelle des Modells in Bild 4.11a festzustellen. Mit steigender Temperatur steigt zu einem das Maximum der Zeitkonstante und zum anderen verschiebt sich dieses Maximum in Richtung niedriger Sauerstofffüllstände, was am größeren nutzbaren Sauerstofffüllstandsbereich liegt. Die Aktivierung des Systems bei höheren Temperaturen ist auch mit einer „Entfaltung“ der Nichtlinearitäten verbunden, d. h. bei höheren 
a)

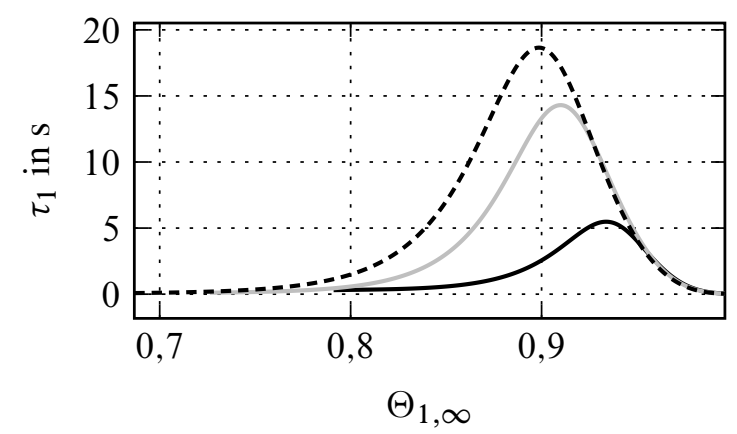

b)

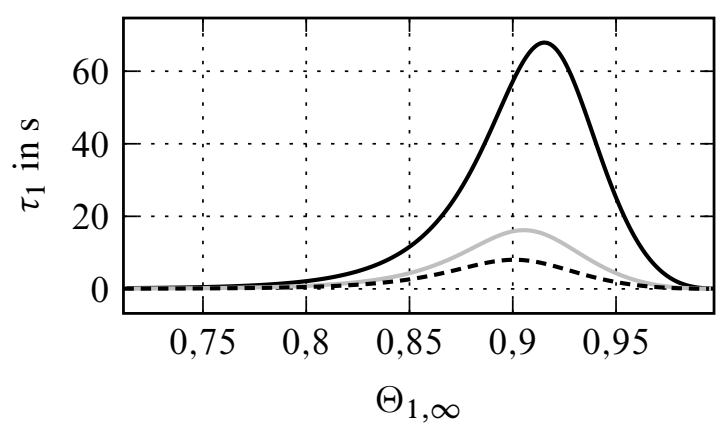

Bild 4.11: Verlauf der Zeitkonstante der ersten Zelle des Modells: a) bei einer Raumgeschwindigkeit von $24600 \mathrm{~h}^{-1}$ für die Temperaturen $700 \mathrm{~K}(-), 810 \mathrm{~K}(-)$ und $920 \mathrm{~K}(----)$; b) bei einer Temperatur von $810 \mathrm{~K}$ für die Raumgeschwindigkeiten $16900 \mathrm{~h}^{-1}(-), 43800 \mathrm{~h}^{-1}$ (—) und $70800 \mathrm{~h}^{-1}(----)$

a)

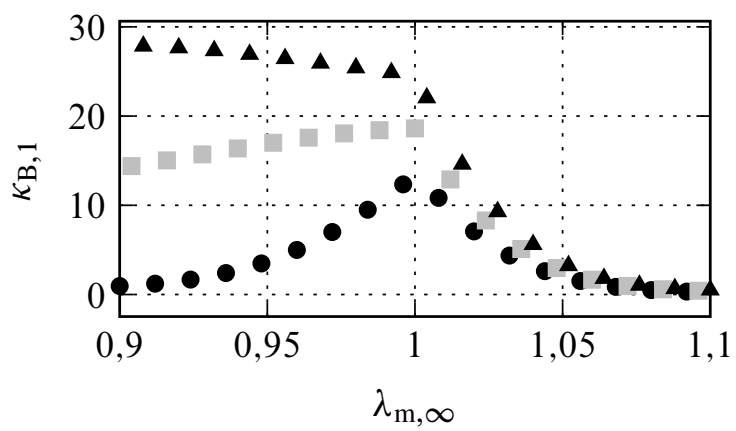

b)

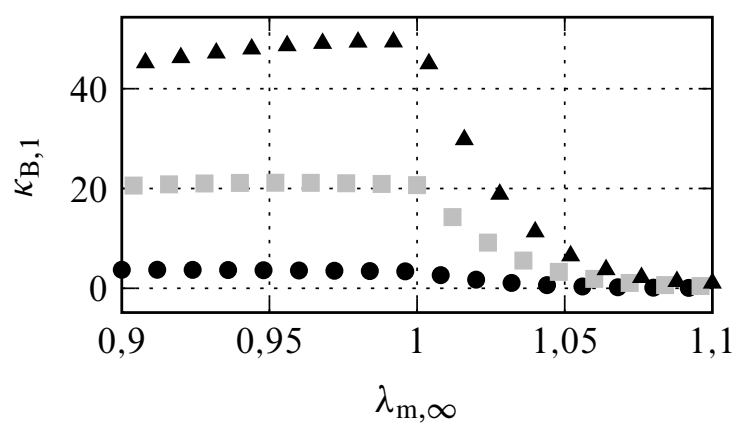

Bild 4.12: Verlauf des Steuerbarkeitsmaßes der ersten Zelle des Modells: a) bei einer Raumgeschwindigkeit von $24600 \mathrm{~h}^{-1}$ für die Temperaturen $700 \mathrm{~K}(\bullet), 810 \mathrm{~K}(\square)$ und $920 \mathrm{~K}(\boldsymbol{\Delta})$; b) bei einer Temperatur von $810 \mathrm{~K}$ für die Raumgeschwindigkeiten $16900 \mathrm{~h}^{-1}(\bullet), 43800 \mathrm{~h}^{-1}$ ( $\square$ ) und $70800 \mathrm{~h}^{-1}(\boldsymbol{\Delta})$

Temperaturen wirken sich die Nichtlinearitäten stärker aus als bei niedrigen Temperaturen. Eine Erhöhung des Abgasmassenstroms bewirkt dagegen eine Abnahme der Zeitkonstante, obwohl die Werte der Gewichtsfunktionen dabei zunehmen, siehe Bild 4.9b. Dies hängt damit zusammen, dass mit steigendem Abgasmassenstrom die Verweilzeit des Gases im Katalysator abnimmt und damit das System schneller bzw. dynamischer wird, was sich in einer kleineren Zeitkonstante äußert.

Steuerbarkeit Der Verlauf des Steuerbarkeitsmaßes der ersten Zelle des Modells ist in Bild 4.12 dargestellt. Bei niedrigen Temperaturen ist eine schlechtere Steuerbarkeit zu beobachten, was vermutlich durch das teilaktivierte System erklärt werden kann. Interessant ist, dass im Bereich des Lambdafensters das Steuerbarkeitsmaß für die niedrigste Temperatur trotzdem ansteigt, was darauf hindeutet, dass der Katalysator in diesem Bereich am besten funktioniert. Höhere Abgasmassenströme führen zu höheren Steuerbarkeitsmaße, was auf höhere Eingangsverstärkungen hindeutet. 
a)

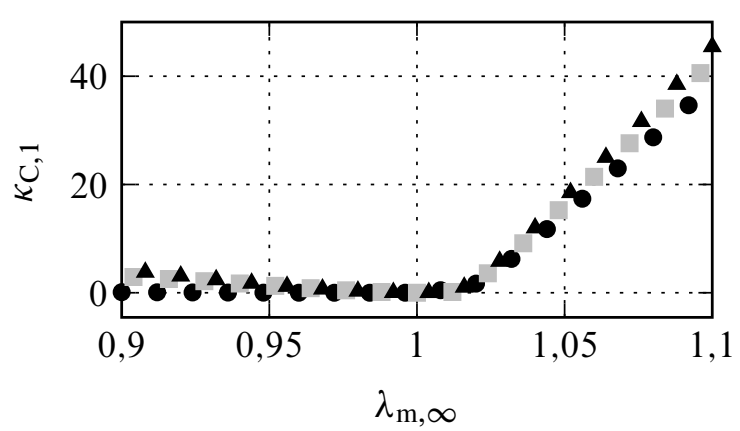

b)

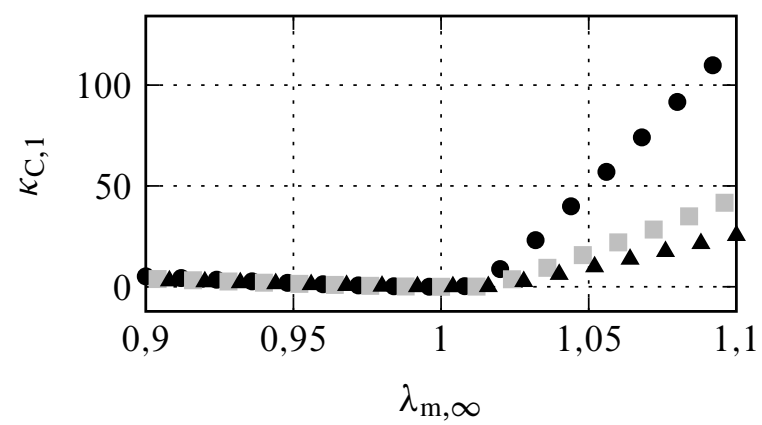

Bild 4.13: Verlauf des Beobachtbarkeitsmaßes der ersten Zelle des Modells: a) bei einer Raumgeschwindigkeit von $24600 \mathrm{~h}^{-1}$ für die Temperaturen $700 \mathrm{~K}(\bullet), 810 \mathrm{~K}(\square)$ und $920 \mathrm{~K}$ (४); b) bei einer Temperatur von $810 \mathrm{~K}$ für die Raumgeschwindigkeiten $16900 \mathrm{~h}^{-1}(\bullet)$, $43800 \mathrm{~h}^{-1}(\square)$ und $70800 \mathrm{~h}^{-1}(\boldsymbol{\Delta})$

Beobachtbarkeit Abschließend ist in Bild 4.13 das Beobachtbarkeitsmaß der ersten Zelle des Modells dargestellt. Die Aktivierung des Systems bei höheren Temperaturen führt generell zu einer geringfügig besseren Beobachtbarkeit, wobei die schlechte Beobachtbarkeit im Lambdafenster erhalten bleibt. Ein steigender Abgasmassenstrom führt dagegen für Lambdawerte $>1 \mathrm{zu}$ einer schlechteren Beobachtbarkeit.

Diskussion Zusammenfassend lässt sich festhalten, dass der Katalysator mit steigender Temperatur aktiver wird und dadurch auch ausgeprägtere Nichtlinearitäten zeigt. Die Steuerbarkeit verbessert sich mit steigender Temperatur, wobei die Steuerbarkeit im Bereich des Lambdafensters auch bei niedrigen Temperaturen erhalten bleibt. Die schlechte Beobachtbarkeit im Bereich des Lambdafensters ist unabhängig von der Temperatur. Aus Sicht der Schadstoffkonvertierung kann dies als ein gutes Zeichen gedeutet werden, da der Katalysator die Lambdaabweichung an seinem Eingang puffern kann und sich das Geschehen am Katalysatoreingang an seinem Ausgang nicht bemerkbar macht. Höhere Abgasmassenströme bzw. Raumgeschwindigkeiten führen dazu, dass sich das Systemverhalten weitestgehend linear verhält und die Systemzustände besser steuerbar sind, ohne dass sich dabei die Beobachtbarkeit stark verändert. Höhere Raumgeschwindigkeiten können also zu einem günstigeren Regelverhalten des Katalysators führen. Eine Verkleinerung des Katalysators, eine der naheliegenden Möglichkeiten der Ressourceneinsparung, steht damit nicht im Widerspruch zu einem besseren Regelverhalten. Die minimale Größe des Katalysators hängt vom erforderlichen Grad der Schadstoffkonvertierung und von der Größe der Totzeiten im Lambdaregelkreis ab, denn der Katalysator soll in der Lage sein, die Lambdaabweichung im Motorabgas zu puffern, bis der Lambdaregelkreis darauf reagieren kann. 


\subsection{Zusammenfassung}

In diesem Kapitel wird die Darstellung des Katalysatormodells mittels Gewichtsfunktionen aus systemtheoretischer und aus chemischer Sicht diskutiert. Die Gewichtsfunktionen erlauben die vorteilhafte Ausnutzung der vorhandenen Systemstruktur und vereinfachen die Systemanalyse, da sie alle Modellparameter vereinen. Wegen ihrer Anschaulichkeit sowohl für Regelungstechniker als auch Chemiker können sie als eine Schnittstelle zwischen diesen beiden Gebieten dienen. Die asymptotische Stabilität des Modells kann mit systemtheoretischen Verfahren direkt am nichtlinearen Modell nachgewiesen werden. Nach der Linearisierung des Modells werden die Verläufe der stationären Arbeitspunkte, der Zeitkonstanten und ausgewählter Strukturmaße in Abhängigkeit des Lambdawertes oder des Sauerstofffüllstandes berechnet und diskutiert. Die Verläufe der Strukturmaße können plausibilisiert werden und vor allem das Steuerbarkeits- und das Beobachtbarkeitsmaß schaffen eine Basis zum quantitativen Vergleich verschiedener Katalysatoren. Es werden die bekannten Probleme bei der Berechnung der Strukturmaße diskutiert und Hinweise für weiterführende Arbeiten gegeben. Abschließend wird der Einfluss der Temperatur und des Abgasmassenstroms auf die zuvor berechneten Größen untersucht. Eine wichtige Erkenntnis dabei ist, dass sich eine Ressourcenoptimierung und ein gutes Regelverhalten nicht zwangsläufig widersprechen müssen. Es muss aber auch hier ein Kompromiss zwischen Ressourceneinsatz und Güte der Abgasnachbehandlung getroffen werden. 


\section{Parameteridentifikation}

Der Einsatz des neu entwickelten Zustandsraummodells setzt in der Praxis voraus, dass seine Parameter bekannt sind. Die Modellparameter werden zwar von den Projektpartnern zur Verfügung gestellt, das Katalysatorverhalten ändert sich jedoch infolge der Alterung im Betrieb. Daher müssen die Modellparameter von Zeit zu Zeit in kleinen Bereichen angepasst werden. Dieses Kapitel widmet sich diesem Thema, oder genauer formuliert, der Identifikation der Modellparameter. Das Modell enthält viele Parameter, deren gleichzeitige Identifikation zu einem Optimierungsproblem großer Dimension führt. Um dies zu vermeiden, werden am Anfang des Kapitels die für das Ein-/Ausgangsverhalten wichtigsten Parameter selektiert. Anschießend wird eine geeignete Lambdatrajektorie gewählt und die wichtigsten Parameter mit Messdaten vom Laborprüfstand identifiziert. Als Optimierungsalgorithmus kommt die in [24] beschriebene Partikel-Schwarm-Optimierung zum Einsatz, die von Kiwic u. a. $[50,51]$ zum selben Zweck wie hier eingesetzt wird. Zur Verifikation der Parameter werden Messungen bei verschiedenen Raumgeschwindigkeiten durchgeführt.

Parameter, die aus der Geometrie des Katalysators berechnet oder aus Tabellen über die Eigenschaften der chemischen Spezies abgelesen werden können, stehen nicht im Fokus der Parameteridentifikation. Die zu identifizierenden Parameter sind jene, die die chemischen Reaktionen auf der katalytischen Oberfläche beschreiben, siehe Tabelle 5.1. Dazu gehören die kinetischen Parameter (die präexponentiellen Faktoren $A_{j}$ und die Aktivierungsenergien $E_{j}$ der drei Reaktionen $j$ ), die thermodynamischen Parameter von Ceroxid (die Entropie- $\Delta S_{\text {Cer }}$ und die Enthalpieänderung $\Delta H_{\text {Cer }}$ ) sowie die Sauerstoffspeicherkapazität $L$.

Zur Modellierung der Temperaturabhängigkeit der Entropie- und der Enthalpieänderung der chemischen Spezies können allgemein Polynome zweiter Ordnung verwendet werden. Die dazugehörigen Parameter der Gasspezies sind Tabellen in der einschlägigen Literatur zu entnehmen. Jedoch

Tabelle 5.1: Liste der zu identifizierenden Modellparameter

\begin{tabular}{ll}
\hline Bezeichnung & Symbol \\
\hline Kinetische Parameter & $A_{1 / 2 / 3}$ \\
& $E_{1 / 2 / 3}$ \\
\hline Thermodynamische Parameter & $\Delta H_{\mathrm{Cer}, 0 / 2}$ \\
& $\Delta S_{\mathrm{Cer}, 0}$ \\
\hline Sauerstoffspeicherkapazität & $L$ \\
\hline
\end{tabular}


müssen die Polynomkoeffizienten für das Ceroxid experimentell ermittelt werden, da sie von den Materialien des Washcoats abhängen [40]. Möller u. a. [64, 65] modellieren die Entropie- und die Enthalpieänderung von Ceroxid nicht in Abhängigkeit der Temperatur, sondern in Abhängigkeit des Sauerstofffüllstandes $\Theta$ durch die folgenden Polynome:

$$
\begin{aligned}
\Delta S_{\mathrm{Cer}} & =\Delta S_{\mathrm{Cer}, 0}, \\
\Delta H_{\mathrm{Cer}} & =\Delta H_{\mathrm{Cer}, 0}+\Delta H_{\mathrm{Cer}, 2} \Theta^{2} .
\end{aligned}
$$

Da hierbei manche Koeffizienten identisch Null sind, wird von vornherein die Anzahl der zu identifizierenden Parameter reduziert.

Für die Bestimmung der Modellparameter werden von den Projektpartnern Messungen am Laborprüfstand durchgeführt. Um die Konzentrationen im Abgas hinter dem Katalysator zu bestimmen, werden dabei Gasanalysatoren verwendet. Da die Optimierung der Parameter hier in kleinen Bereichen stattfindet, wird wie von Kiwic u. a. vom Gebrauch der Gasanalysatoren Abstand genommen und nur die Signale zweier Breitband-Lambdasonden verwendet: eine vor und eine hinter dem Katalysator.

In [124] werden Verfahren zur Parameteridentifikation des Katalysators untersucht. Die in dem folgenden Abschnitt durchgeführten analytischen Sensitivitätsanalysen werden dort numerisch und unter Betrachtung größerer Variationsbereiche der Parameter durchgeführt.

\subsection{Sensitivitätsanalyse}

Die Parameteroptimierung, die der Alterung im Betrieb oder kleinen produktionsbedingten Unterschieden zwischen den Katalysatoren der Projektpartner Rechnung trägt, muss eventuell nicht alle Parameter in Tabelle 5.1 berücksichtigen. Um die Parameter zu bestimmen, die am stärksten den Lambdawert hinter dem Katalysator und die Systemdynamik beeinflussen, kann eine Sensitivitätsanalyse durchgeführt werden. Zum Beispiel wählen Rao u. a. [80] und Ramanathan und Sharma [79] die zu optimierenden Katalysatorparameter anhand ihrer Sensitivität und der Systemkenntnis der Autoren. Im Gegensatz zum numerischen Ansatz von Rao u. a. werden im Folgenden die Sensitivitäten analytisch berechnet. Beim Einsetzen der numerischen Werte wird der zweite Motorarbeitspunkt verwendet, siehe Anhang A.1.

Es wird zwischen der globalen und der lokalen Sensitivitätsanalyse unterschieden. Bei der globalen Sensitivitätsanalyse wird der Einfluss von Parametern bei einer Variation über ihren gesamten Definitionsbereich auf die Ausgangsgröße ermittelt [95]. Die Durchführung dieser Analyse ist beim nichtlinearen Katalysatormodell jedoch kompliziert, sodass hier eine lokale Sensitivitätsanalyse durchgeführt wird. Dabei wird der Einfluss von kleinen Parameteränderungen an einem bestimmten Arbeitspunkt auf die Ausgangsgröße ermittelt. Es wird weiter zwischen der absoluten Sensitivität

$$
S_{x, p_{i}}=\frac{\partial x}{\partial p_{i}}
$$


und der relativen Sensitivität unterschieden [110]:

$$
S_{x, p_{i}}^{\mathrm{rel}}=\frac{\partial x}{\partial p_{i}} \frac{p_{i}}{x}
$$

In Gleichung (5.3) und (5.4) bezeichnet $x$ die zu untersuchende Größe und $p_{i}$ die Parameter, deren Einfluss ermittelt wird.

Parametereinfluss auf den Lambdawert Im ersten Teil der Analyse werden die relativen Sensitivitäten bezogen auf den Lambdawert $\lambda_{\text {su }}$ hinter dem Katalysator ermittelt. Um die Sensitivitäten analytisch berechnen zu können, wird ein Katalysatormodell mit nur einer Zelle betrachtet. In diesem Fall ergibt sich nach Gleichung (4.13) und einigen mathematischen Umformungen der folgende Ausdruck für den Lambdawert $\lambda_{\mathrm{su}}$ :

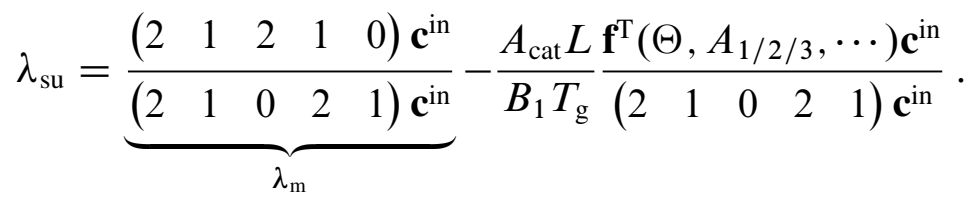

Der erste Term in Gleichung (5.5) entspricht dem Lambdawert $\lambda_{\mathrm{m}}$ im Motorabgas vor dem Katalysator. Der zweite Term enthält im Zähler den Ausdruck $\mathbf{f}^{\mathrm{T}} \mathbf{c}^{\mathrm{in}}$, der die Ableitung des Sauerstofffüllstandes darstellt, siehe Gleichung (3.39). Er hängt zum einen vom Sauerstofffüllstand $\Theta$ und zum anderen von den Parametern in Tabelle $5.1 \mathrm{ab}$. An der Gleichung ist sehr gut zu erkennen, dass in den stationären Arbeitspunkten, d. h. für $\dot{\Theta}=\mathbf{f}^{\mathrm{T}} \mathbf{c}^{\text {in }}=0$, die Lambdawerte vor und hinter dem Katalysator gleich sind. Da die Eingangskonzentrationen $\mathbf{c}^{\text {in }}$ von den Parametern $p_{i}$ unabhängig sind und der Vektor $\mathbf{f}^{\mathrm{T}}$ mit den Gewichtsfunktionen nur im Zähler vorkommt, ergibt sich der folgende einfache Ausdruck für die relative Sensitivität:

$$
S_{\lambda_{\mathrm{su}}, p_{i}}^{\mathrm{rel}}=-\frac{A_{\mathrm{cat}} L}{B_{1} T_{\mathrm{g}}} \frac{1}{\left(\begin{array}{lllll}
2 & 1 & 0 & 2 & 1
\end{array}\right) \mathbf{c}^{\mathrm{in}}} \frac{\partial \mathbf{f}^{\mathrm{T}}\left(\Theta, A_{1 / 2 / 3}, \cdots\right)}{\partial p_{i}} \mathbf{c}^{\mathrm{in}} \frac{p_{i}}{\lambda_{\mathrm{su}}} .
$$

Gleichung (5.6) gilt auch für die relative Sensitivität der Sauerstoffspeicherkapazität $L$, da der Ausdruck $\mathbf{f}^{\mathrm{T}} \mathbf{c}^{\mathrm{in}}$, der in der partiellen Ableitung von Gleichung (5.5) nach $L$ auftaucht, an den stationären Arbeitspunkten identisch Null ist.

In Bild 5.1 sind die relativen Sensitivitäten $S_{\lambda_{\text {su }}, p_{i}}^{\text {rel }}$ der Parameter in Tabelle 5.1 dargestellt. Die Sensitivitäten werden an den stationären Arbeitspunkten ausgewertet und sind gegen den Sauerstofffüllstand aufgetragen. Es fällt auf, dass die Sensitivitäten vor allem an den Rändern des Arbeitsbereichs größere Werte annehmen und die thermodynamischen Parameter von Ceroxid, die die Entropie- $\Delta S_{\text {Cer }}$ und die Enthalpieänderung $\Delta H_{\text {Cer }}$ parametrieren, einen stärkeren Einfluss besitzen. Verglichen mit der kleinsten Sensitivität der thermodynamischen Parameter, dieser von $\Delta S_{\mathrm{Cer}, 0}$, sind die Sensitivitäten der Aktivierungsenergien $E_{1 / 2 / 3}$ der Reaktionen um etwa den Faktor 15 kleiner und die Sensitivitäten der restlichen Parameter sogar um etwa den Faktor 30 kleiner. 
a)

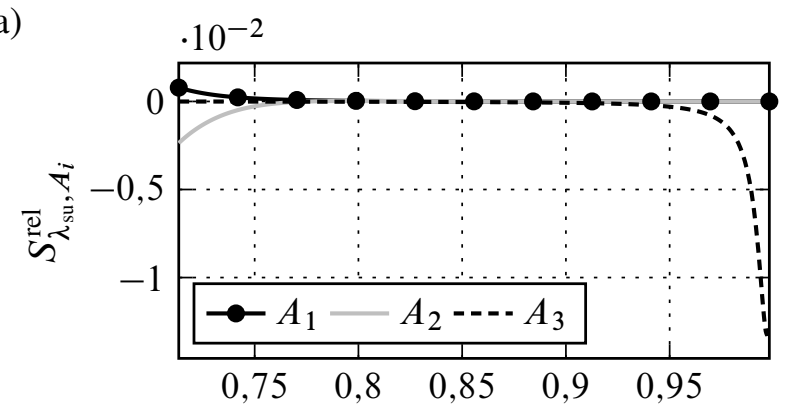

c)

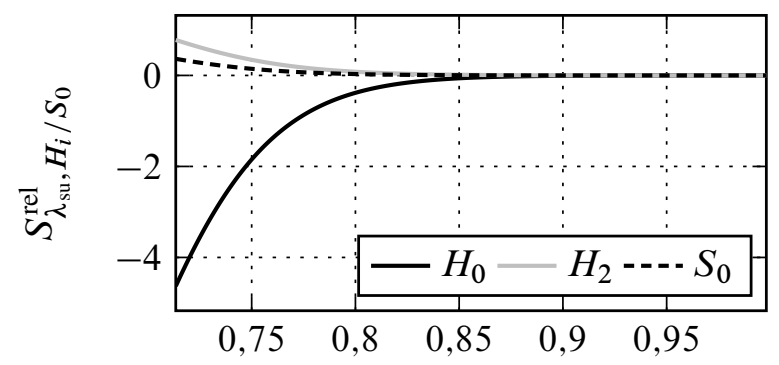

$\Theta$ b)

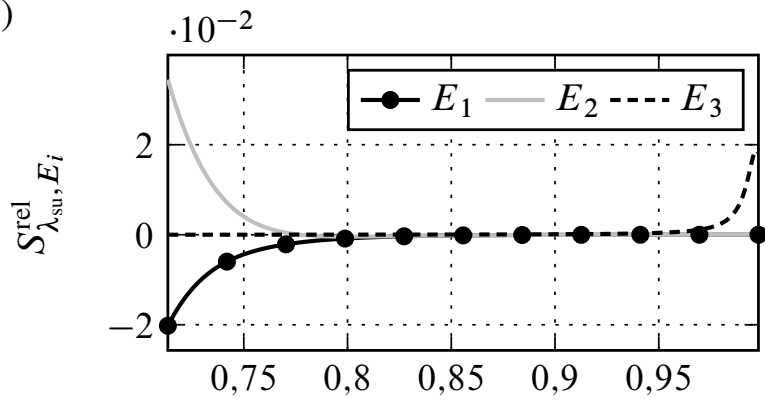

d)

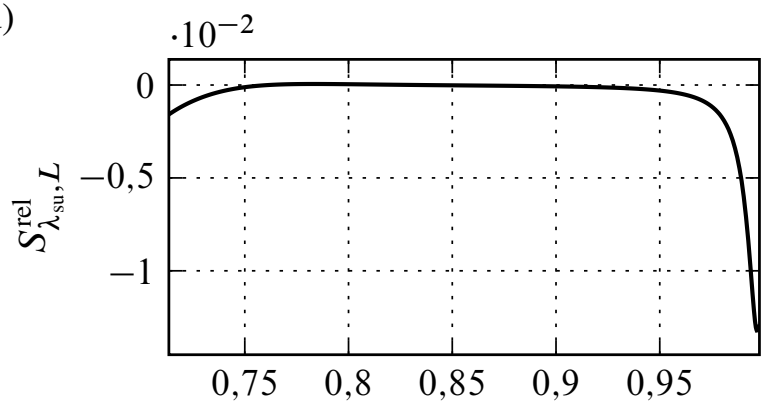

$\Theta$

Bild 5.1: Relative Sensitivität $S_{\lambda_{\text {su }}, p_{i}}^{\text {rel }}$ der Parameter in Tabelle 5.1: a) präexponentielle Faktoren; b) Aktivierungsenergien; c) thermodynamische Parameter von Ceroxid; d) Sauerstoffspeicherkapazität $L$

Parametereinfluss auf die Systemdynamik Der zweite Teil der Analyse umfasst die Berechnung der relativen Sensitivitäten bezogen auf die Zeitkonstante $\tau_{1}$, die dem invertierten und negierten Eigenwert $a_{1}$ entspricht. Der einzige Eigenwert bei der Diskretisierung des Modells in einer Zelle entspricht dem ersten Element in der ersten Zeile der Matrix des linearisierten Systems, siehe Gleichung (4.10), und lautet:

$$
a_{1}=\frac{\partial \mathbf{f}^{\mathrm{T}}\left(\Theta, A_{1 / 2 / 3}, \cdots\right)}{\partial \Theta} \mathbf{c}^{\text {in }} .
$$

Anwenden von Gleichung (5.4) auf den Ausdruck für die Zeitkonstante $\tau_{1}=-1 / a_{1}$ liefert die relative Sensitivität:

$$
S_{\tau_{1}, p_{i}}^{\mathrm{rel}}=\frac{\partial \tau_{1}}{\partial p_{i}} \frac{p_{i}}{\tau_{1}}=\frac{\partial a_{1}}{\partial p_{i}} \frac{1}{a_{1}^{2}} \frac{p_{i}}{\tau_{1}}=\frac{\partial^{2} \mathbf{f}^{\mathrm{T}}\left(\Theta, A_{1 / 2 / 3}, \cdots\right)}{\partial \Theta \partial p_{i}} \mathbf{c}^{\mathrm{in}} p_{i} \tau_{1} .
$$

Für ihre Berechnung ist es notwendig, dass die fünf Gewichtsfunktionen sowohl nach dem Sauerstofffüllstand $\Theta$ als auch nach den Parametern $p_{i}$ abgeleitet werden. Obwohl die symbolischen Ausdrücke dabei deutlich in die Länge wachsen, können die symbolischen Ableitungen innerhalb weniger Sekunden an einem aktuellen PC berechnet werden. In dieser Arbeit wird dafür MuPAD ${ }^{\circledR}$ eingesetzt, ein Computeralgebrasystem, das in der Symbolic Math Toolbox ${ }^{\mathrm{TM}}$ von MATLAB ${ }^{\circledR}$ enthalten ist. MATLAB ${ }^{\circledR}$ bietet die Möglichkeit, das Katalysatormodell symbolisch zu implementieren, alle benötigten Ableitungen zu berechnen und daraus ausführbare Funktionen zu generieren, die numerische Werte berechnen. Beim Einsetzen der numerischen Werte in Gleichung (5.8) ergeben sich jedoch nicht in jedem Fall die richtigen Vorzeichen, was auf das zweifache Ab- 
a)

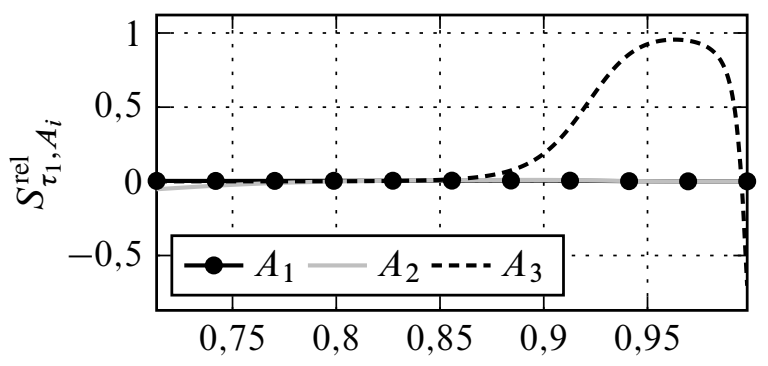

c)

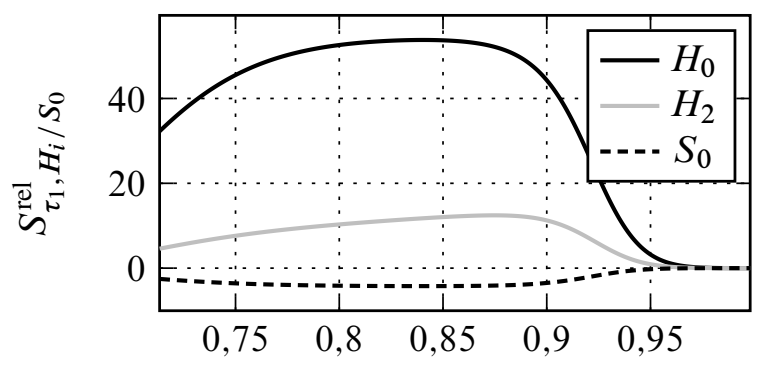

$\Theta$ b)

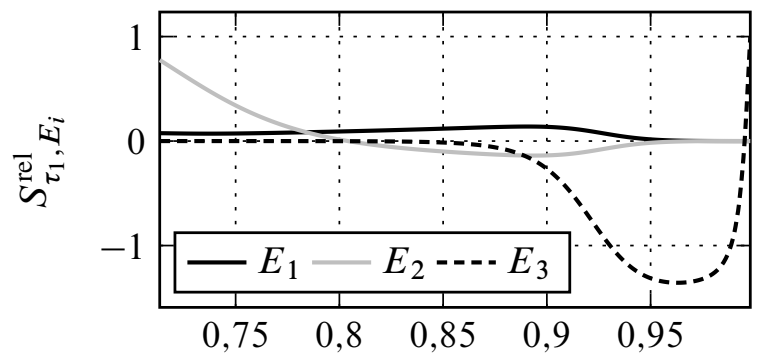

d)

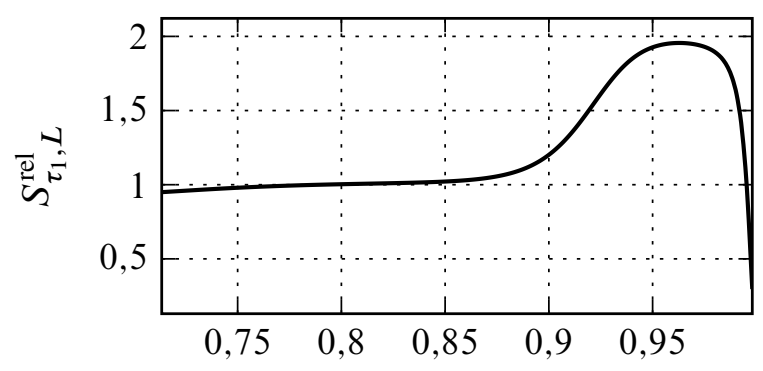

$\Theta$

Bild 5.2: Relative Sensitivität $S_{\tau_{1}, p_{i}}^{\text {rel }}$ der Parameter in Tabelle 5.1: a) präexponentielle Faktoren; b) Aktivierungsenergien; c) thermodynamische Parameter von Ceroxid; d) Sauerstoffspeicherkapazität $L$

leiten zurückzuführen ist. Aus diesem Grund werden zuerst die Beträge der relativen Sensitivitäten berechnet und in einem zweiten Schritt die Vorzeichen experimentell bestimmt.

In Bild 5.2 sind die relativen Sensitivitäten $S_{\tau_{1}, p_{i}}^{\text {rel }}$ der Parameter in Tabelle 5.1 in Abhängigkeit des Sauerstofffüllstandes dargestellt. Wie beim ersten Teil der Sensitivitätsanalyse werden die numerischen Werte an den stationären Arbeitspunkten eingesetzt. Wieder weisen die drei thermodynamischen Parameter den stärksten Einfluss auf, wobei $\Delta H_{\mathrm{Cer}, 0}$ den größten Wert annimmt. Während bei der ersten Analyse die größten Sensitivitäten an den Rändern des Arbeitsbereichs auftreten, schlagen die Sensitivitäten jetzt innerhalb des Arbeitsbereichs aus. Die nächstgrößere Sensitivität weist die Sauerstoffspeicherkapazität $L$ auf. Dies zeigt sehr anschaulich, dass eine größere Sauerstoffspeicherkapazität mit höheren Zeitkonstanten einhergeht. Die kinetischen Parameter der Reaktion (R3), $A_{3}$ und $E_{3}$, weisen im Vergleich zu den Parametern der anderen zwei Reaktionen einen stärkeren Einfluss auf. Dies ist einleuchtend, da die Reaktion mit $\mathrm{O}_{2}$ unmittelbar in Verbindung mit der Sauerstoffspeicherung in Verbindung steht und diese Eigenschaft bei der Modellbildung in Kapitel 3 als bestimmend für die Systemdynamik angenommen wird. Bei niedrigen Sauerstofffüllständen nimmt die Sensitivität von $E_{2}$ zu und damit der Einfluss der Reaktion (R2), die $\mathrm{H}_{2} \mathrm{O}$ und $\mathrm{H}_{2}$ berücksichtigt, auf die Systemdynamik. Vergleicht man die Absolutwerte von $\Delta S_{\mathrm{Cer}, 0}$ und $E_{2}$, so haben die kinetischen Parameter einen mindestens um den Faktor Zwei kleineren Einfluss auf die Systemdynamik. 


\subsection{Identifikationstrajektorie und Identifikationsverfahren}

Die Sensitivitätsanalyse im vorherigen Abschnitt ergibt, dass die drei thermodynamischen Parameter den größten Einfluss auf den Lambdawert hinter dem Katalysator und auf die Zeitkonstante haben. Die Sauerstoffspeicherkapazität, die kinetischen Parameter der Reaktion (R3) und die Aktivierungsenergie der Reaktion (R2) kommen als Nächstes bei der Wahl der zu optimierenden Parameter in Frage. Die Sauerstoffspeicherkapazität hängt jedoch selbst von den thermodynamischen Parametern von Ceroxid ab, sodass von der gleichzeitigen Optimierung dieser vier Parameter abgesehen wird. Da die Parameteroptimierung in kleinen Bereichen stattfinden soll, wird auf die explizite Optimierung von $L$ verzichtet. Aufgrund ihres kleineren Einflusses und motiviert durch interne Gespräche mit den Projektpartnern werden des Weiteren die kinetischen Parameter nicht optimiert. Das heißt, die Parameteroptimierung beschränkt sich auf die drei thermodynamischen Parameter $\Delta H_{\mathrm{Cer}, 0}, \Delta H_{\mathrm{Cer}, 2}$ und $\Delta S_{\mathrm{Cer}, 0}$. Der empfohlene Variationsbereich liegt bei $\pm 2 \%$.

Identifikationstrajektorie Die Lambdatrajektorie, die am Laborprüfstand nachgefahren wird, um Messdaten für den Optimierungsalgorithmus zu generieren, muss das System ausreichend anregen. Den Verläufen der Sensitivitäten der thermodynamischen Parameter in Bild 5.1 und Bild 5.2 ist zu entnehmen, dass bei der Identifikationsmessung der Sauerstoffspeicher bis zum Minimum entleert und fast bis zum Maximum befüllt werden sollte. An den stationären Arbeitspunkten sind die Lambdawerte vor und hinter dem Katalysator gleich, siehe Gleichung (5.5), sodass durch die Parameteroptimierung kein Einfluss auf stationäre Abweichungen zwischen den Mess- und den Simulationsverläufen genommen werden kann. Kiwic u. a. [50, 51] setzen eine Lambdatrajektorie ein, die im ersten Teil aus einer Schwingung mit steigender Amplitude und steigender Periode und im zweiten Teil aus Lambdasprüngen besteht. Dabei ist die Trajektorie so ausgelegt, dass der Sauerstoffspeicher am Anfang die kleinen Lambdaabweichungen puffern kann und es erst später zu Emissionsdurchbrüchen kommt. Die hier eingesetzte Trajektorie beruht auf der von Kiwic u. a. und ist in Bild 5.3 beispielhaft dargestellt. Die Trajektorie wird je nach Raumgeschwindigkeit leicht verändert. Sie ist so ausgelegt, dass die Lambda-Zeit-Flächen der Halbschwingungen quadratisch mit der Zeit steigen. Der Soll-Lambdawert $\lambda_{\mathrm{w}}$ am Laborprüfstand setzt sich additiv aus einem stationären Lambdawert und der Trajektorie in Bild 5.3 zusammen. Der stationäre Lambdawert wird so gewählt, dass sich ein mittlerer Sauerstofffüllstand einstellt. Auf seine Bestimmung wird im nächsten Abschnitt eingegangen.

Optimierungsalgorithmus Aufgrund der starken Nichtlinearitäten und der potentiell großen Anzahl der zu optimierenden Parameter ziehen Kiwic u. a. für die Optimierung der Parameter des Katalysatormodells die Partikel-Schwarm-Optimierung einem Gradientenverfahren vor. Die Partikel-Schwarm-Optimierung ist ein numerischer Algorithmus, der die Parameter findet, die eine gegebene Zielfunktion minimieren. Der Algorithmus ist vom biologischen Schwarmverhalten inspiriert. Die Zielfunktion kann nichtlinear sein und beliebig vielen linearen und nichtlinearen Beschränkungen unterliegen. Bei der Optimierung der Parameter des Katalysatormodells wird als Zielfunktion die über die Länge der Identifikationstrajektorie integrierte Abweichung zwischen dem gemessenen und dem simulierten Lambdawert hinter dem Katalysator verwendet. In [24] ist 


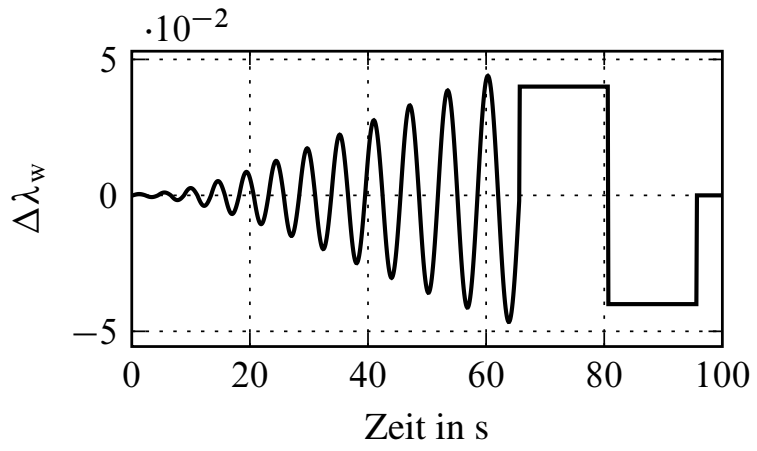

Bild 5.3: Lambdatrajektorie $\Delta \lambda_{\mathrm{w}}$ zur Parameteroptimierung des Katalysatormodells

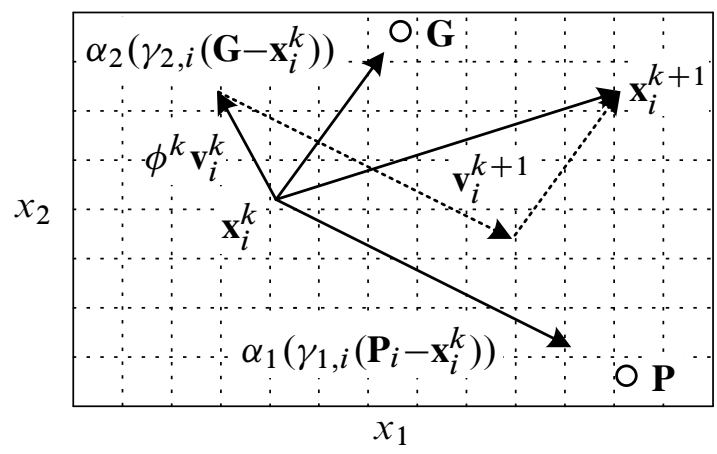

Bild 5.4: Zur Veranschaulichung der Grundidee der Partikel-Schwarm-Optimierung, aus [24]

eine ausführliche Beschreibung des in MATLAB ${ }^{\circledR}$ implementierten Algorithmus zu finden. Im Folgenden wird nur kurz anhand Bild 5.4 die Grundidee der Methode geschildert.

Am Anfang der Optimierung wird die gewünschte Anzahl an Partikeln zufällig im Parametersuchraum verteilt. Jedes Partikel $i$ zeichnet sich durch seine Position $\mathbf{x}_{i}^{k}$ und seine Bewegungsgeschwindigkeit $\mathbf{v}_{i}^{k}$ im aktuellen Optimierungsschritt $k$ aus. Die Bewegungsgeschwindigkeit $\mathbf{v}_{i}^{k+1}$ im nächsten Optimierungsschritt ist eine Funktion der aktuellen Bewegungsgeschwindigkeit $\mathbf{v}_{i}^{k}$, der Differenz zwischen der aktuellen Position $\mathbf{x}_{i}^{k}$ und der besten Position $\mathbf{P}_{i}$ und der Differenz zwischen der aktuellen Position $\mathbf{x}_{i}^{k}$ und der besten Position des Schwarmes G:

$$
\mathbf{v}_{i}^{k+1}=\phi^{k} \mathbf{v}_{i}^{k}+\alpha_{1}\left(\gamma_{1, i}\left(\mathbf{P}_{i}-\mathbf{x}_{i}^{k}\right)\right)+\alpha_{2}\left(\gamma_{2, i}\left(\mathbf{G}-\mathbf{x}_{i}^{k}\right)\right)
$$

Die Trägheit $\phi$ und die Beschleunigungskonstanten $\alpha_{1 / 2}$ dienen zur Abstimmung des Algorithmus, während $\gamma_{1 / 2}$ gleichverteilte Zufallszahlen aus dem geschlossenen Intervall $[0,1]$ sind. Die Position des Partikels im nächsten Optimierungsschritt ergibt sich nach folgender Gleichung:

$$
\mathbf{x}_{i}^{k+1}=\mathbf{x}_{i}^{k}+\mathbf{v}_{i}^{k+1}
$$

Eventuelle Verletzungen der Beschränkungen mit der neuen Position können auf verschiedene Weise behandelt werden [24].

Weiterhin wird eine Normierung der Parameter durchgeführt, sodass alle Parameter eine ähnliche Sensitivität aufweisen. Dies ist insbesondere dann wichtig, wenn z. B. gleichzeitig die präexponentiellen Faktoren und die Aktivierungsenergien der Reaktionen optimiert werden [50]. Da die drei thermodynamischen Parameter auf die gleiche Weise in die Systemgleichungen eingehen, wird nur eine Normierung auf das geschlossene Intervall $[-1,1]$ vorgenommen.

\subsection{Parameteridentifikation mit Messdaten}

Die für die Parameteroptimierung benötigten Messdaten werden am Laborprüfstand aufgenommen. In Anhang A.2 sind Informationen zum Aufbau und zur Ausstattung des Laborprüfstandes 


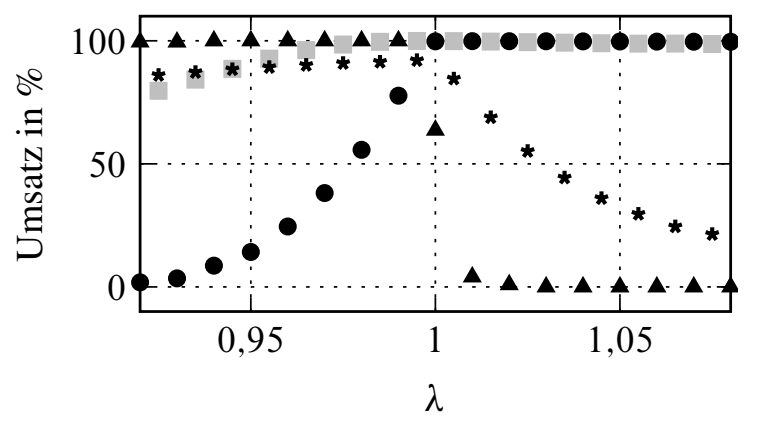

Bild 5.5: Gemessene Konvertierungsraten des Katalysators in Abhängigkeit vom Lambdawert bei einer Temperatur von $773 \mathrm{~K}$ und einer Raumgeschwindigkeit von $100000 \mathrm{~h}^{-1}$ : $\mathrm{CO}(\bullet)$, $\mathrm{C}_{3} \mathrm{H}_{6}(\square), \mathrm{NO}(\boldsymbol{\Delta}), \mathrm{O}_{2}(*)$

zu finden. Der verwendete Katalysator weist einen Trägerkörper aus Cordierit mit einer Zelldichte von $600 \mathrm{cpsi}$ und eine $\mathrm{Pt} / \mathrm{Pd} / \mathrm{Rh}$-Edelmetallbeladung von $10 \mathrm{~g} / \mathrm{ft}^{3}$ im Verhältnis $2 / 3 / 5$ auf. Er ist 16 Stunden bei $985^{\circ} \mathrm{C}$ hydrothermal, d. h. in einer Atmosphäre mit $10 \% \mathrm{H}_{2} \mathrm{O}, 10 \% \mathrm{O}_{2}$ und $80 \% \mathrm{~N}_{2}$, gealtert worden. Bis auf die unterschiedliche Alterung entspricht der Katalysator jenem von Möller u. a. [64, 65]. Wenn keine weiteren Angaben zu den Versuchsrandbedingungen gemacht werden, beträgt die Gastemperatur am Katalysatoreingang $773 \mathrm{~K}$ und die Raumgeschwindigkeit $100000 \mathrm{~h}^{-1}$. Verglichen mit den Motorarbeitspunkten, siehe Anhang A.1, liegt die Raumgeschwindigkeit am Laborprüfstand etwas höher. Dies liegt vor allem daran, dass der verwendete Katalysator eine sehr hohe Schadstoffkonvertierung aufweist und durch die höhere Raumgeschwindigkeit die Randbedingungen absichtlich verschärft werden.

Mittlerer Sauerstofffüllstand Die Lambdatrajektorie zur Parameteridentifikation aus dem vorherigen Abschnitt addiert zu einem konstanten Lambdawert ergibt den Soll-Lambdawert der Massendurchflussregler am Laborprüfstand. An dieser Stelle stellt sich die Frage, von welchem konstanten Lambdawert ausgegangen werden soll. In der Praxis wird ein mittlerer Sauerstofffüllstand und eine damit verbundene Spannung der Sprung-Lambdasonde hinter dem Katalysator von etwa 600-650 mV angestrebt [4, 51, 64]. Der Sauerstofffüllstand kann in der Praxis jedoch nicht gemessen werden, ${ }^{1}$ sodass die Zuordnung zwischen dem Lambdawert vor dem Katalysator und dem Sauerstofffüllstand nicht bekannt ist. Direkt anzunehmen, dass z. B. $625 \mathrm{mV}$ einem mittleren Sauerstofffüllstand entsprechen, kann irreführend sein, da es je nach Katalysatortechnologie und Kraftstoffzusammensetzung Unterschiede geben kann. Daher wird im Folgenden die Spannung der Sprung-Lambdasonde und damit auch der Lambdawert vor dem Katalysator, die zu einem mittleren Sauerstofffüllstand führen, experimentell ermittelt. Dabei wird davon ausgegangen, dass ein mittlerer Sauerstofffüllstand einen Durchbruch der Schadstoffe CO und NO ähnlich gut dämpfen kann. Es werden diese zwei Spezies gewählt, da sich ihre Konvertierungsraten außerhalb des Lambdafensters stark verändern, siehe Bild 5.5.

Bei den Experimenten wird zuerst die Spannung der Sprung-Lambdasonde hinter dem Katalysator auf einen Wert im Bereich von 550 bis $750 \mathrm{mV}$ eingestellt und zum sich dabei einstellenden

\footnotetext{
${ }^{1}$ Eine Ausnahme stellt hier der neuartige, hochfrequenzbasierte Ansatz von Schödel u. a. [89] dar.
} 


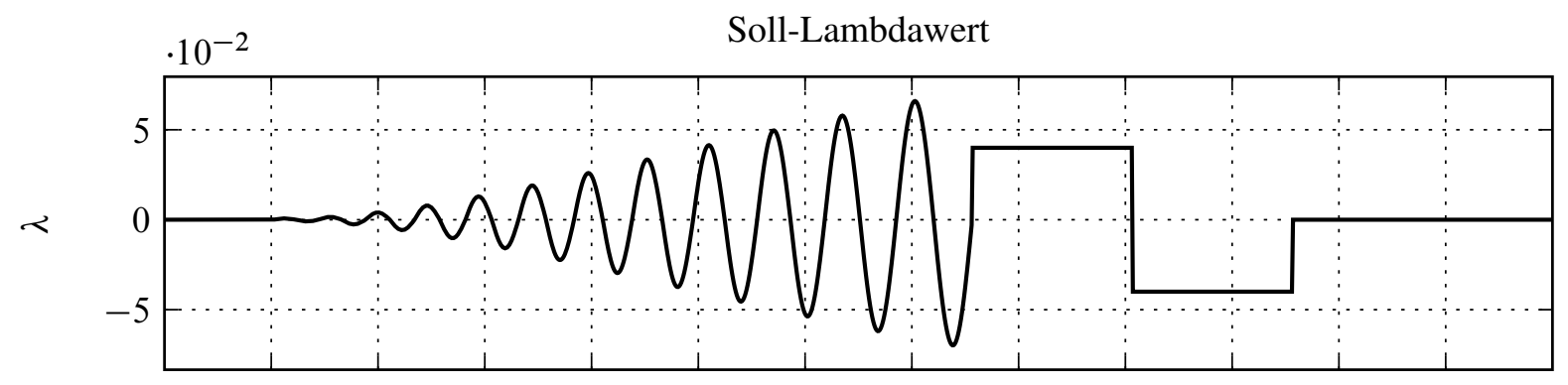

NO- und CO-Konzentrationen
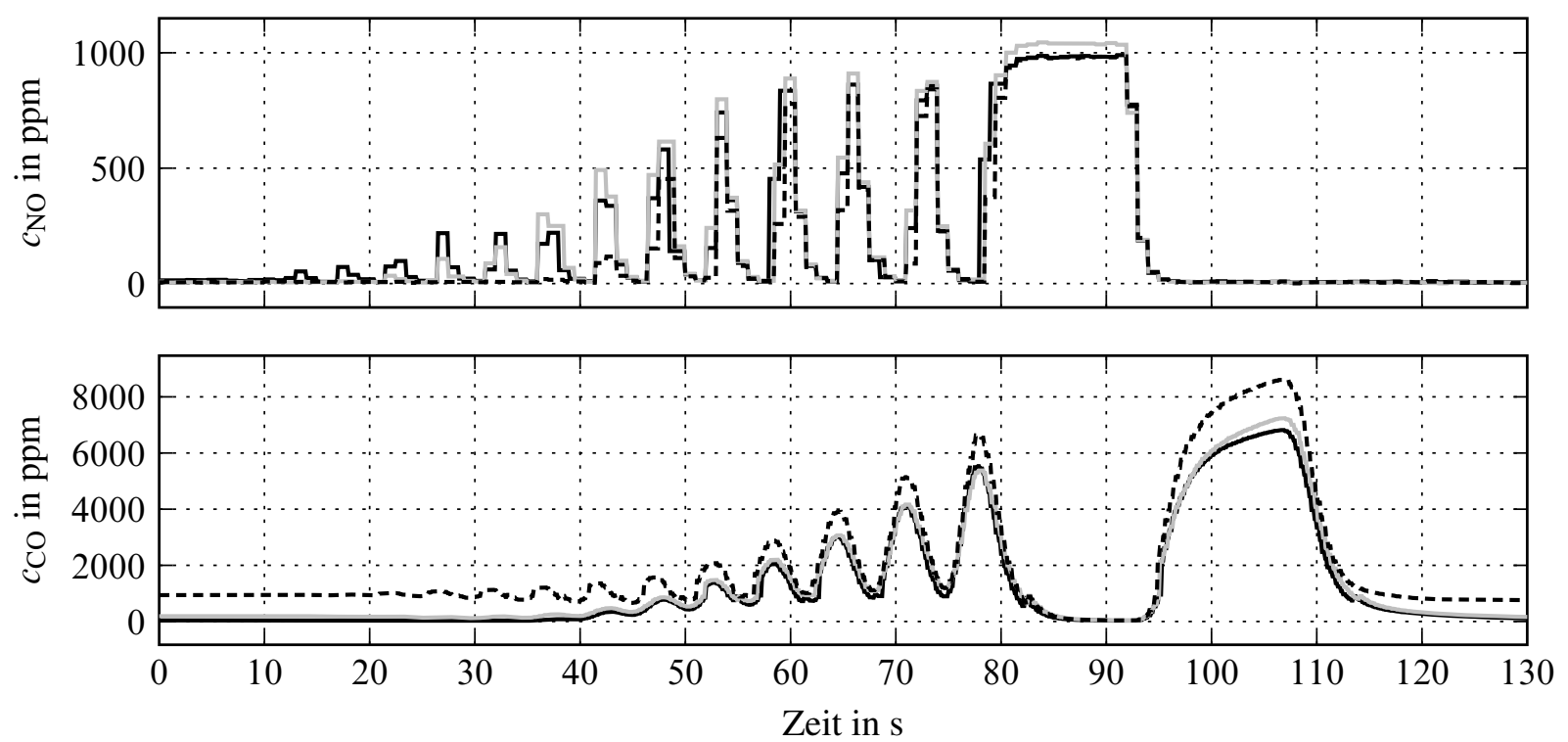

Bild 5.6: NO- und CO-Konzentrationen während der Identifikationsmessung für verschiedene Spannungen der Sprung-Lambdasonde hinter dem Katalysator: $550 \mathrm{mV}(-), 650 \mathrm{mV}(-)$, $750 \mathrm{mV}(--\cdot)$

Lambdawert vor dem Katalysator die Identifikationstrajektorie addiert. Je nachdem, ob der Sauerstoffüllstand unterhalb oder oberhalb der Mitte des Arbeitsbereichs liegt, bricht mit steigender Lambdaamplitude entweder CO oder NO früher und stärker durch. Von Experiment zu Experiment wird der Spannungswert jeweils um $50 \mathrm{mV}$ verändert. In Bild 5.6 sind der Übersichtlichkeit halber nur die bei drei Experimenten gemessenen CO- und NO-Konzentrationen dargestellt. Wie zu erwarten, korrelieren höhere CO-Emissionen mit höheren Spannungswerten und höhere NOEmissionen mit niedrigeren Spannungswerten. Die Differenz zwischen den CO-Konzentrationen bei 550 und $650 \mathrm{mV}$ ist deutlich kleiner als die zwischen 650 und $750 \mathrm{mV}$, sodass ein Spannungswert niedriger als $650 \mathrm{mV}$ keine nennenswerten Vorteile bei den CO-Emissionen mit sich bringt. Zwar sind immer höhere Spannungswerte mit immer späteren NO-Emissionsdurchbrüchen verbunden, bei $650 \mathrm{mV}$ wird jedoch ein guter Kompromiss zwischen den CO- und NO-Emissionen erreicht. Daher wird dieser Spannungswert mit einem mittleren Sauerstofffüllstand assoziiert.

Mit dem Emissionsmodell des Laborprüfstandes, siehe Anhang A.2, ergibt sich ein Sauerstofffüllstandsbereich für das Katalysatormodell von etwa 0,75 bis 1, siehe auch Bild 4.4 und Bild 4.10. Der mittlere Sauerstofffüllstand wird durch die Mittelung der Sauerstofffüllstände der drei Mo- 
Soll-Lambdawert

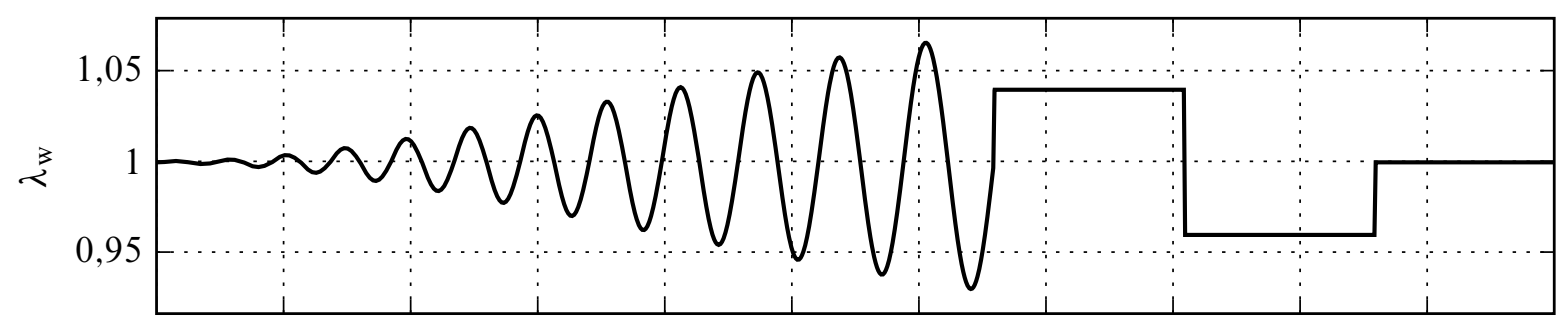

Gemessener und simulierter Lambdawert
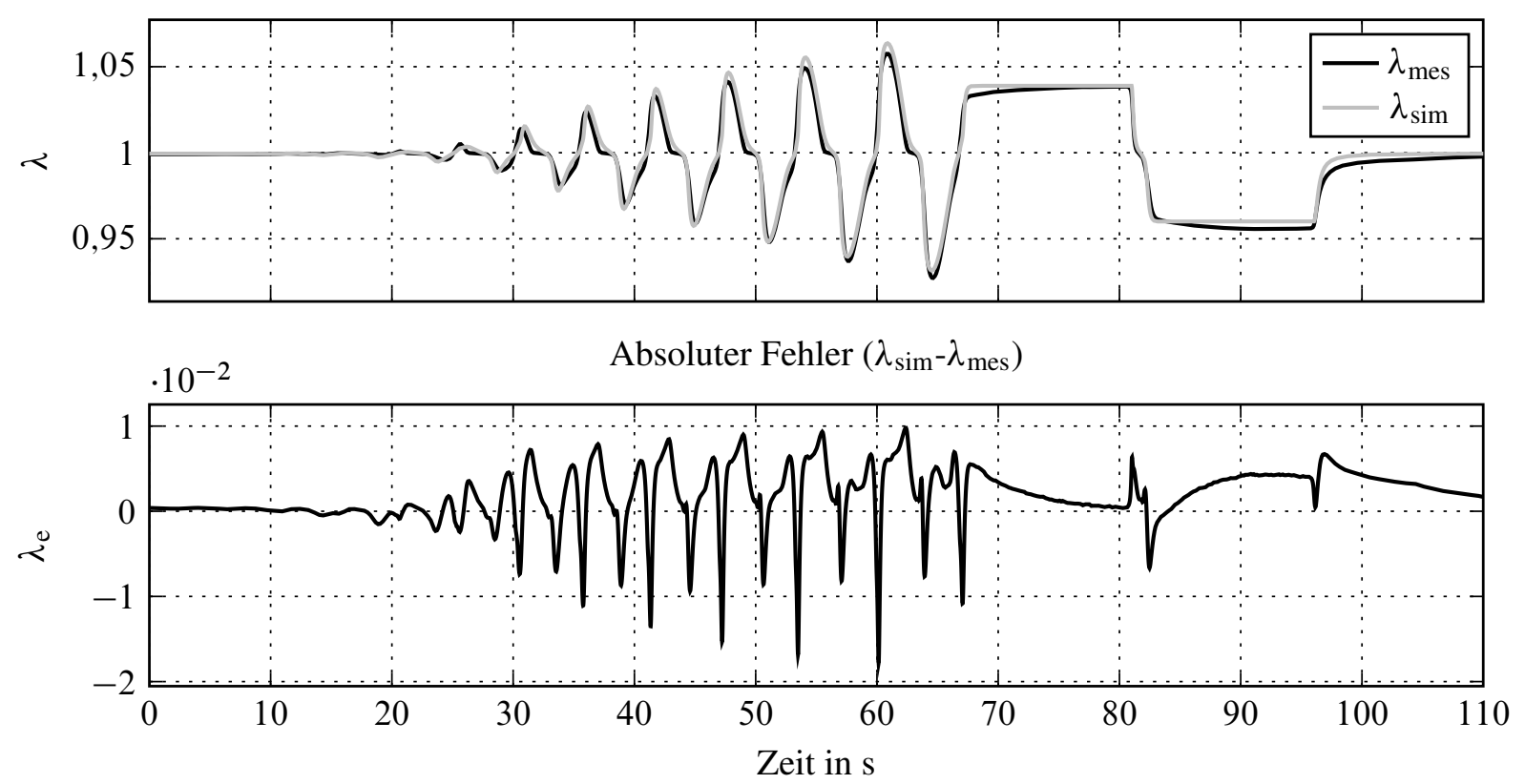

Bild 5.7: Parameteridentifikation: Vergleich zwischen Simulation und Messung nach der Parameteroptimierung

dellzellen im Lambdaintervall von 0,98 bis 1,02 festgelegt. Das Lambdaintervall wird so gewählt, dass die Kurven der stationären Sauerstofffüllstände in etwa punktsymmetrisch zum mittleren Sauerstofffüllstand verlaufen. Für die Versuchsrandbedingungen ergibt sich der folgende Arbeitspunkt des Katalysatormodells:

$$
\boldsymbol{\Theta}_{\infty}=\left(\begin{array}{lll}
0,89 & 0,91 & 0,91
\end{array}\right)
$$

Optimierung der Parameter Die Identifikationsmessung wird am Laborprüfstand durchgeführt. Das aufgenommene Signal der Breitband-Lambdasonde hinter dem Katalysator wird anschließend vorwärts und rückwärts, d.h. ohne Phasenverschiebung, gefiltert. In der Simulationsumgebung wird die Identifikationsmessung unter Berücksichtigung des Emissionsmodells des Laborprüfstandes, der Gastransportzeit und der Dynamik der Breitband-Lambdasonde hinter dem Katalysator implementiert. Es zeigt sich, dass nicht mehr als 120 Partikel und zwei Generationen notwen- 
Soll-Lambdawert

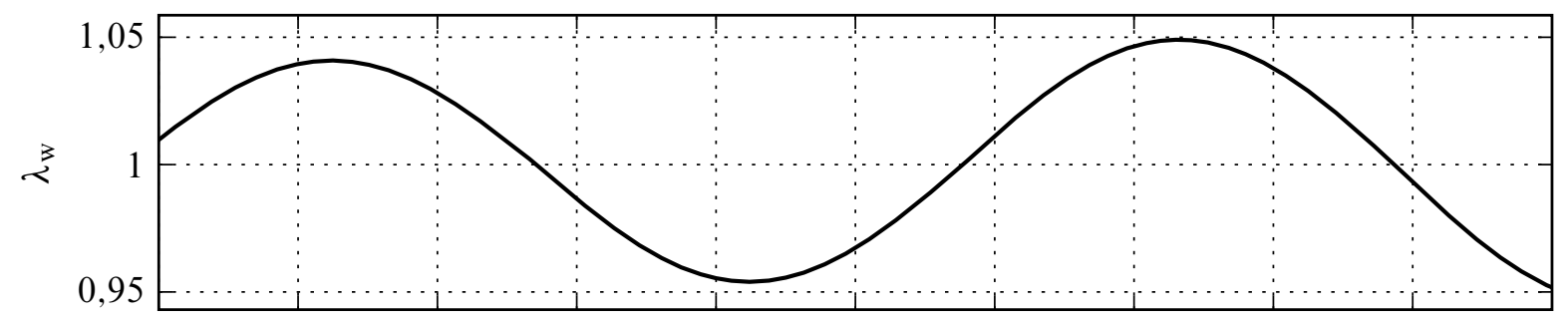

Gemessener und simulierter Lambdawert
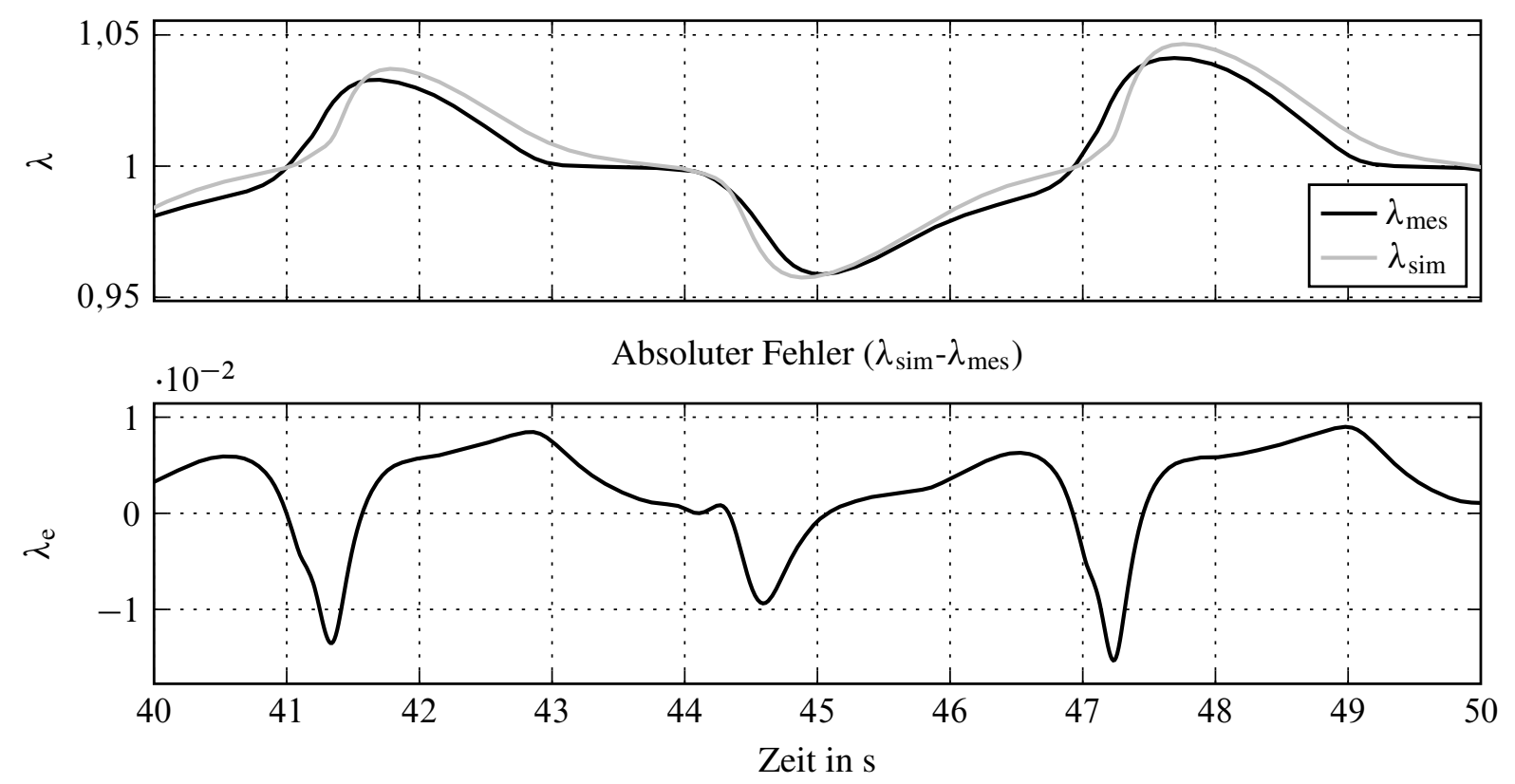

Bild 5.8: Parameteridentifikation: Vergleich zwischen Simulation und Messung nach der Parameteroptimierung. Vergrößerung des Bereichs zwischen 40 und $50 \mathrm{~s}$.

dig sind, um zu einem lokalen Minimum der Zielfunktion zu gelangen. In Anbetracht des kleinen Variationsbereichs der Parameter und der hohen Partikelanzahl ist davon auszugehen, dass das gefundene Minimum auch das globale Minimum im Variationsbereich darstellt. In Bild 5.7 sind die Verläufe des Soll-Lambdawerts, des gemessenen und des simulierten Lambdawertes hinter dem Katalysator nach der Parameteroptimierung sowie des absoluten Fehlers zwischen der Simulation und der Messung dargestellt. Es ist gut zu erkennen, dass die kleinen Amplituden der Lambdaabweichung am Anfang der Identifikationsmessung durch den Sauerstoffspeicher gepuffert werden können. Amplituden erst ab 1,5-2 \% machen sich am Katalysatorausgang bemerkbar. Obwohl das Katalysatormodell auf einigen Vereinfachungen und nur drei Reaktionsgleichungen beruht, stimmen die Messung und die Simulation qualitativ gut überein, wenn auch der absolute Fehler bis zu 1,5\% beträgt. In Bild 5.8 ist eine Vergrößerung des Bereichs von 40 bis 50 s dargestellt. Der gemessene und der simulierte Lambdaverlauf sind sehr ähnlich, allerdings mit Zeitversatz. Für Lambdawerte kleiner Eins eilt der simulierte dem gemessenen Verlauf vor und für Lambdawerte größer Eins kehrt sich das Verhalten um. Dieses Verhalten lässt sich mit einer Optimierung der Parameter auch in einem größeren Bereich als die angesetzten $\pm 2 \%$ nicht verbessern. Die gute 
Soll-Lambdawert

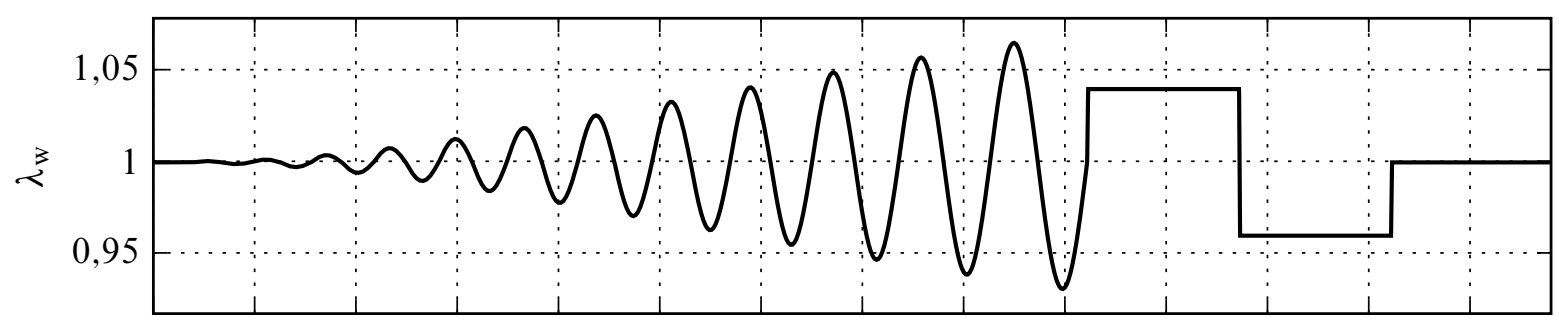

Gemessener und simulierter Lambdawert

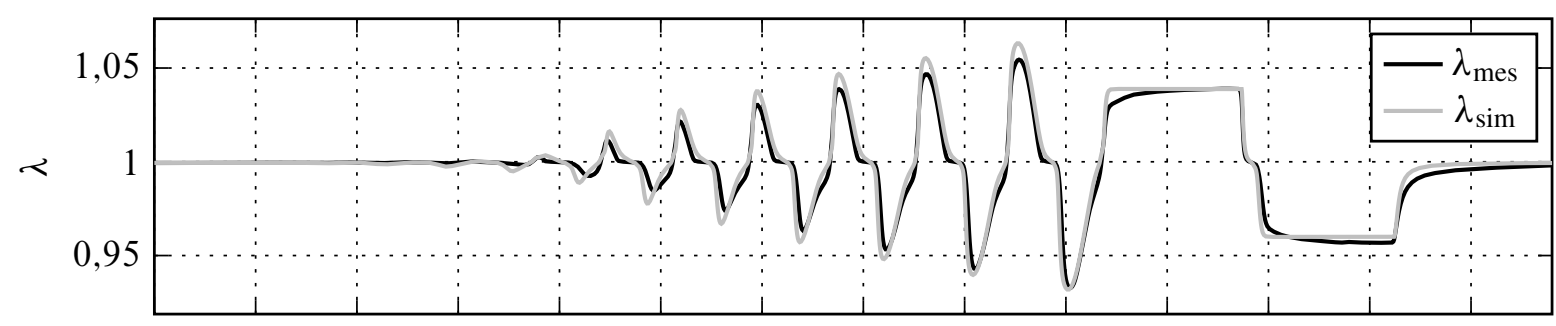

Absoluter Fehler $\left(\lambda_{\text {sim }}-\lambda_{\text {mes }}\right)$

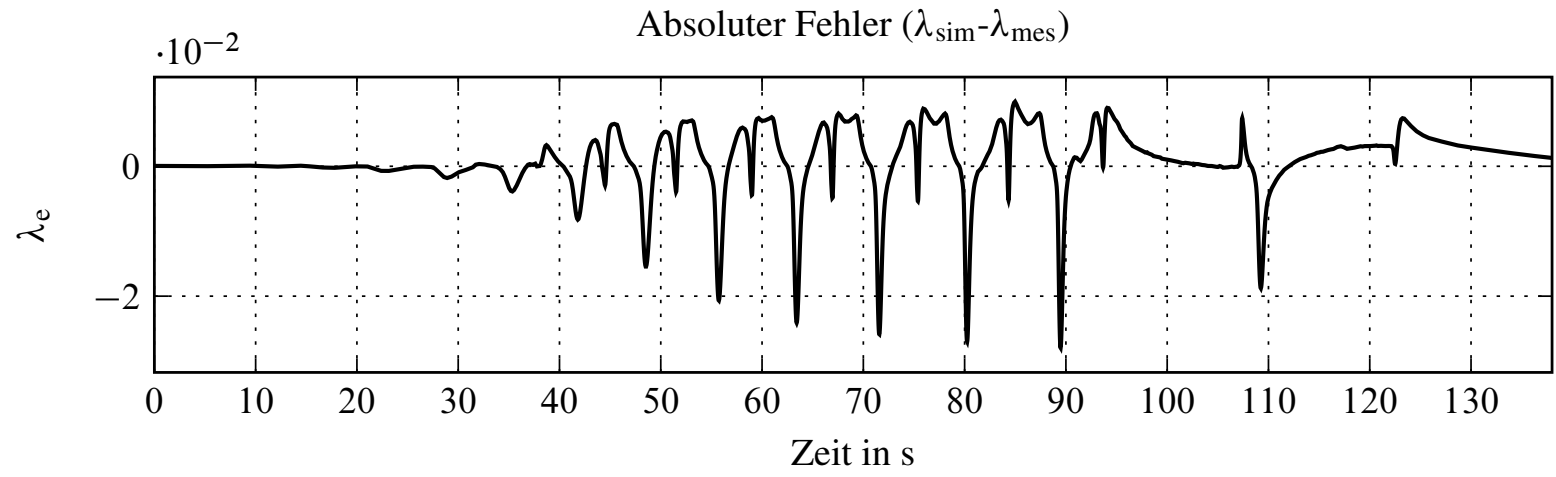

Bild 5.9: Parameterverifikation: Vergleich zwischen Simulation und Messung nach der Parameteroptimierung. Die Temperatur beträgt $773 \mathrm{~K}$ und die Raumgeschwindigkeit $75000 \mathrm{~h}^{-1}$.

Übereinstimmung zwischen der Messung und der Simulation deutet des Weiteren darauf hin, dass die Arbeitspunkte des realen Katalysators und des Katalysatormodells geeignet gewählt sind.

Verifikation der Parameter Zur Verifikation der Parameter wird wieder die Identifikationstrajektorie verwendet. Allerdings wird die Periode der Schwingung am Anfang der Trajektorie so verändert, dass sie auch bei einer Raumgeschwindigkeit von 75000 und $150000 \mathrm{~h}^{-1}$ sinnvoll eingesetzt werden kann. Da die Raumgeschwindigkeit einen signifikanten Einfluss auf die Systemdynamik hat, siehe Abschnitt 4.5, kann durch ihre Variation überprüft werden, ob die Parameter allgemein oder nur in Bezug auf die Raumgeschwindigkeit von $100000 \mathrm{~h}^{-1}$ optimiert worden sind. Die dazugehörigen Verifikationsmessungen sind in Bild 5.9 und Bild 5.10 dargestellt. Eine gute Übereinstimmung zwischen den Mess- und den Simulationsverläufen ist gegeben, wenn auch der absolute Fehler bei der niedrigen Raumgeschwindigkeit angestiegen ist. Das liegt vor allem an dem größeren zeitlichen Versatz zwischen dem gemessenen und dem simulierten Lambdaverlauf. Interessant ist, dass dieser zeitliche Versatz bei der höchsten Raumgeschwindigkeit am kleinsten ausfällt. Dies deutet darauf hin, dass dieses Verhalten eventuell in Verbindung mit dy- 
Soll-Lambdawert

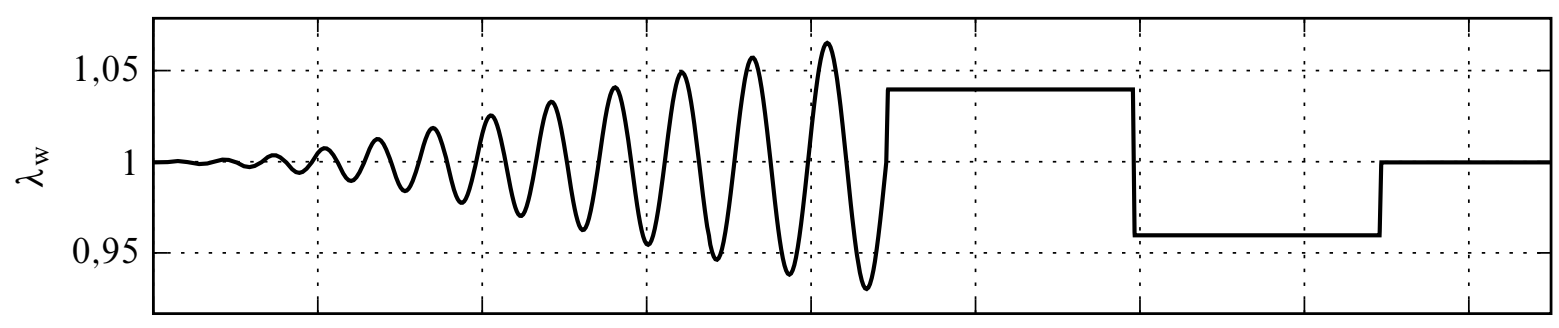

Gemessener und simulierter Lambdawert

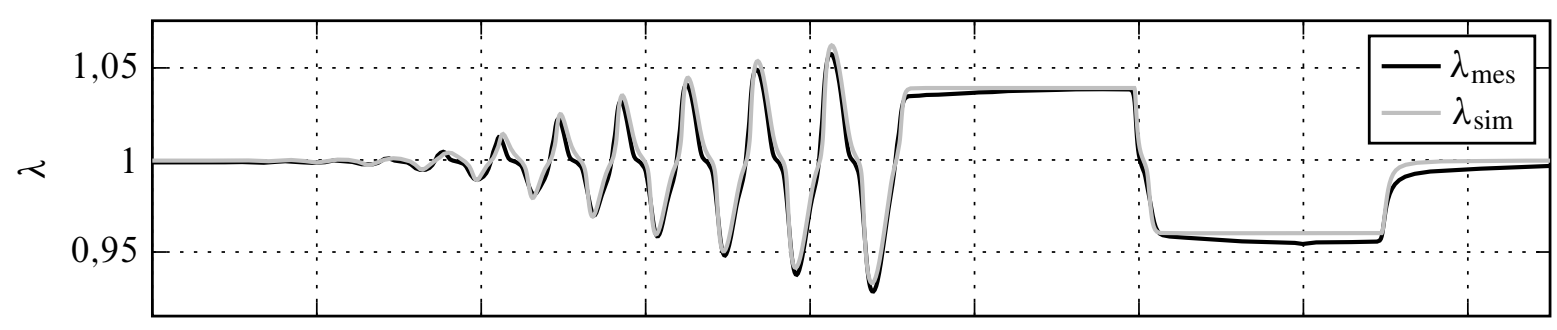

Absoluter Fehler $\left(\lambda_{\text {sim }}-\lambda_{\text {mes }}\right)$

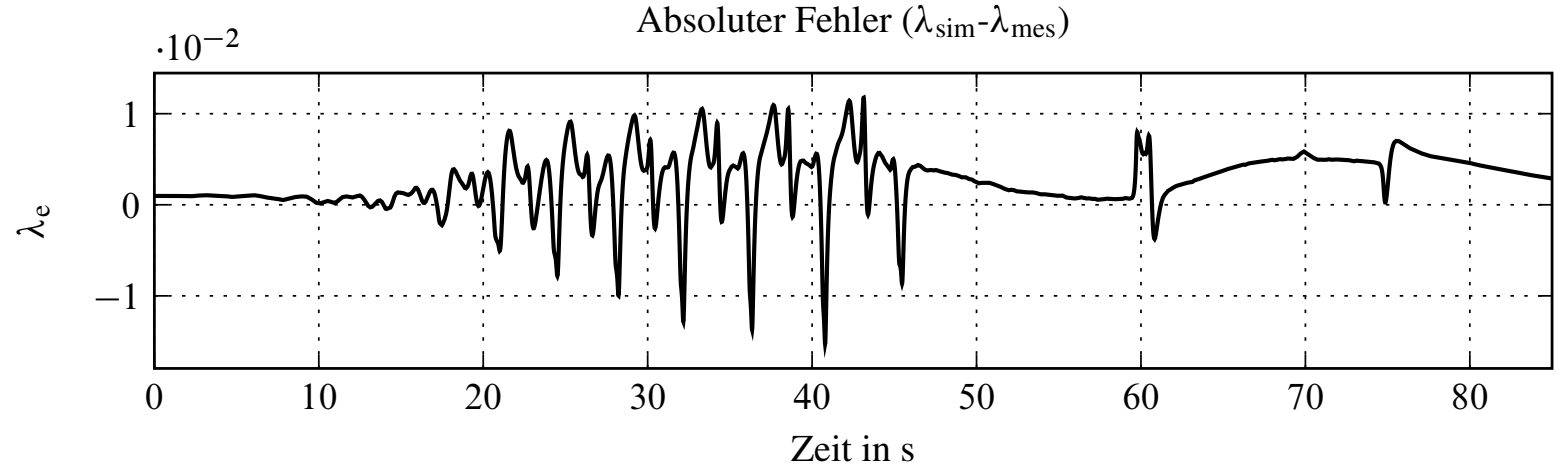

Bild 5.10: Parameterverifikation: Vergleich zwischen Simulation und Messung nach der Parameteroptimierung. Die Temperatur beträgt $773 \mathrm{~K}$ und die Raumgeschwindigkeit $150000 \mathrm{~h}^{-1}$.

namischen Vorgängen steht, die bei der Modellbildung vernachlässigt worden sind und bei der höchsten Raumgeschwindigkeit nicht ins Gewicht fallen.

\subsection{Zusammenfassung}

In diesem Kapitel wird die Parameteridentifikation des Katalysatormodells vorgestellt. Auf Basis einer analytischen Sensitivitätsanalyse, die durch die neue Modelldarstellung erleichtert wird, werden die zu optimierenden Parameter gewählt. Es zeigt sich, dass die thermodynamischen Parameter der Sauerstoffspeicherspezies den größten Einfluss auf den Lambdawert hinter dem Katalysator und auf die Systemdynamik besitzen. Dieses Ergebnis kommt nicht unerwartet, da sich das Modell auf die Sauerstoffspeicherdynamik fokussiert. Die Identifikationstrajektorie wird angelehnt an die Literatur gewählt, jedoch leicht modifiziert. Zur Minimierung der Zielfunktion wird eine Partikel-Schwarm-Optimierung gewählt. Der Lambdawert, der zu einem mittleren Sauerstofffüllstand führt, wird experimentell ermittelt und abschließend werden die eigentlichen 
Identifkations- und Verifikationsmessungen durchgeführt. Es zeigt sich, dass die optimierten Parameter auch bei unterschiedlichen Raumgeschwindigkeiten zu einer guten Übereinstimmung zwischen den simulierten und den gemessenen Lambdaverläufen führen. 


\section{Regelung des Katalysators}

Nach einer Literaturübersicht zur Regelung von Drei-Wege-Katalysatoren wird in diesem Kapitel basierend auf den Ergebnissen der Systemanalyse ein Regelungskonzept vorgeschlagen. Das entwickelte Zustandsraummodell des Katalysators erlaubt den systematischen Entwurf von linearen und nichtlinearen Reglern und drei Abschnitte in diesem Kapitel widmen sich diesem Thema. In Anlehnung an die klassische Sauerstofffüllstandsregelung wird zuerst mittels exakter Ein-/Ausgangslinearisierung ein Regler für den Sauerstofffüllstand im hinteren Bereich des Katalysators entworfen. Anschließend wird für den in der Literatur oft anzutreffenden LQ-Regler eine modellbasierte Auslegung vorgestellt, die direkt die Konvertierungsraten der Spezies berücksichtigt. Es werden zwei weitere nichtlineare Regler vorgestellt und alle entworfenen Regler werden in der Simulationsumgebung miteinander verglichen sowie ihre spezifischen Vor- und Nachteile diskutiert. Der mittels exakter Ein/Ausgangslinearisierung entworfene Regler für den Sauerstofffüllstand und der LQ-Regler werden am Laborprüfstand implementiert. Hierfür werden abschließend Messergebnisse für drei Testszenarien gezeigt und diskutiert. Einzelne Aspekte dieses Kapitels sind in Stanchev u. a. [127, 128, 129] zu finden.

Wie in Abschnitt 1.1.4 erläutert, bleibt nach dem heutigen Stand der Technik die Dynamik des Katalysators im Regelkreis zur Abgasnachbehandlung weitgehend unberücksichtigt. In einigen Forschungsarbeiten, in denen die Katalysatordynamik berücksichtigt wird, z. B. diejenigen von Fiengo u. a. [30] sowie Muske und Peyton Jones [69], wird auch die Frage nach einer alternativen Sensorkonfiguration gestellt, nämlich nach der Nutzung jeweils einer Breitband-Lambdasonde vor und hinter dem Katalysator. Problematisch dabei ist jedoch, dass Breitband-Lambdasonden für eine Platzierung im Motorabgas entwickelt und entsprechend kalibriert sind. Bei einer exakten Kalibrierung für das Motorabgas sind die Messfehler bei einer Platzierung hinter dem Katalysator proportional zu den Differenzen der $\mathrm{H}_{2}$ - und der $\mathrm{NO}_{2}$-Konzentrationen vor und hinter dem Katalysator. Dieses Problem kann nicht durch eine Rekalibrierung der Lambdasonde gelöst werden, da die Konzentrationen im Abgas hinter dem Katalysator dynamisch von seinem Zustand abhängig sind. Die Modellierung der Messfehler ist möglich, erfordert jedoch ein Katalysatormodell, das die entsprechenden Gaskonzentrationen richtig wiedergibt. Eventuell ist es dieser Problematik geschuldet, dass sich die Nutzung zweier Breitband-Lambdasonden bisher nicht durchgesetzt hat. Die starke Abhängigkeit des Signals der Breitband-Lambdasonde von der Abgaszusammensetzung spiegelt die Tatsache wider, dass der Lambdawert nicht direkt, sondern indirekt über den Partialdruck der Gasspezies ermittelt wird [71].

Die meisten aus der Literatur bekannten Regelungskonzepte für den Katalysator zielen darauf ab, den Sauerstofffüllstand auf einem mittleren Wert zu halten, siehe z. B. die Arbeiten von Auck- 
enthaler [4], Möller [64] und Kiwic u. a. [50, 51]. Wie in Abschnitt 5.3 bereits erläutert, kann die Lambdaabweichung vom stöchiometrischen Wert im Motorabgas gepuffert und die Schadstoffemissionen gering gehalten werden, solange der Sauerstoffspeicher einen mittleren Wert aufweist. Dieses Regelziel ist vereinfachend, da im Katalysator nicht nur Sauerstoff sondern auch andere Gasspezies gespeichert werden [40]. Da jedoch die Sauerstoffspeicherung die dominante Dynamik beschreibt, kann dieses Regelziel signifikante Verbesserungen im Emissionsverhalten gegenüber Lambda-Eins-Regelstrategien erzielen. Bei Lambda-Eins-Regelstrategien wird ausschließlich der Lambdawert vor dem Katalysator auf den stöchiometrischen Wert geregelt. Im Allgemeinen wird eine Kaskadenstruktur aus der unterlagerten Lambdaregelung und einer überlagerten Emissionsregelung verwendet, siehe z. B. die Arbeiten von Fiengo u.a., Auckenthaler, Möller sowie Tomforde u. a. [102]. Dabei basiert der Emissionsregler auf einem Katalysatormodell und korrigiert den Sollwert des Lambdaregelkreises. Im Folgenden werden einige Beispiele für die Schätzung des Sauerstofffüllstandes und den Entwurf des Emissionsreglers aus der Literatur genannt. Die Korrektur des Offsets der Breitband-Lambdasonde vor dem Katalysator mit dem Signal der Sprung-Lambdasonde hinter dem Katalysator kann dabei als die einfachste Emissionsregelung angesehen werden.

Beim Entwurf des Emissionsreglers wird der unterlagerte Lambdaregelkreis in der Literatur oft nicht berücksichtigt, was an der deutlich langsameren Sauerstoffspeicherdynamik im Bereich des Lambdafensters liegt. Es ist jedoch sinnvoll, zumindest die Totzeit im Lambdaregelkreis zu berücksichtigen, wie z. B. beim Reglerentwurf von Balenovic [5].

Schätzung des Sauerstofffüllstandes Unabhängig von der konkreten Methode zur Regelung des Sauerstofffüllstandes wird eine Schätzung für seinen aktuellen Wert benötigt. Hierfür werden je nach Katalysatormodell unterschiedliche Verfahren eingesetzt. Für das phänomenologische Modell mit konzentrierten Parametern von Muske und Peyton Jones, siehe Gleichung (3.3), wird eine Moving-Horizon-Schätzung basierend auf der Methode der kleinsten Quadrate implementiert. Für das ähnliche Katalysatormodell von Schallock u. a. [87] wird ein Luenberger-Beobachter entworfen, wobei neben dem Sauerstofffüllstand noch die Lambdaabweichung $\lambda_{\mathrm{d}}$ im Motorabgas geschätzt wird. Fiengo u. a. [30, 31] entwirfen für ein weiteres phänomenologisches, eindimensionales Katalysatormodell ein Erweitertes Kalman Filter. Das Entwurfsmodell berücksichtigt die Totzeit des Motors, die Lambdaabweichung, den Sauerstofffüllstand, die Transportzeit der Abgase bis zur Lambdasonde hinter dem Katalysator sowie die zwei Lambdasonden, sodass ein Modell sechster Ordnung vorliegt. Für die in drei Zellen diskretisierten, physikochemischen Modelle von Auckenthaler und Möller werden ebenfalls Erweiterte Kalman Filter entworfen. Des Weiteren wird für das in 20 Zellen diskretisierte, physikochemische Modell von Kiwic u. a. ein Open-LoopBeobachter eingesetzt.

Neben der Schätzung des Sauerstofffüllstandes spielt die Schätzung der Parameter des Katalysatormodells eine wichtige Rolle, da infolge der Alterung die Sauerstoffspeicherkapazität abnimmt. Auckenthaler schätzt die Sauerstoffspeicherkapazität mittels des Erweiterten Kalman Filters. Balenovic ermittelt den Parameter $k_{\mathrm{d}}$ und die Funktionen $f(\Theta)$ und $g(\Theta)$ in Gleichung (3.5) anhand 


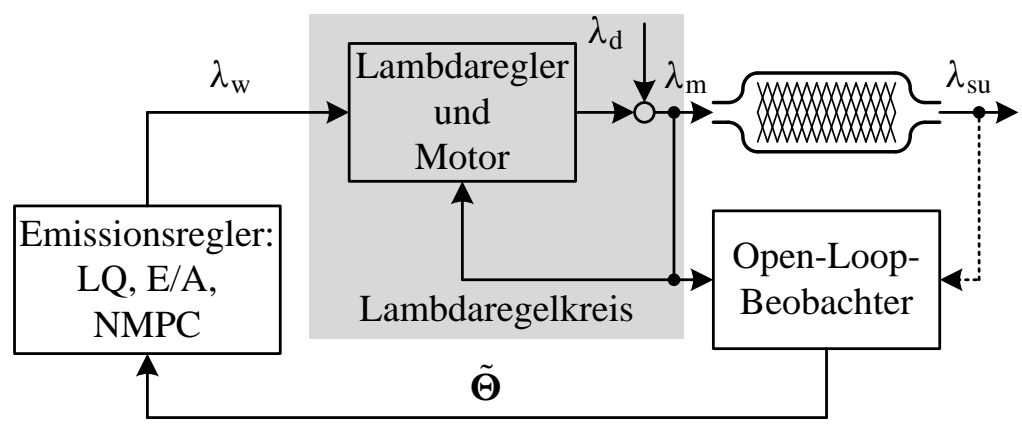

Bild 6.1: Strukturbild des Regelungskonzeptes für den Katalysator bestehend aus dem unterlagerten Lambdaregelkreis und einer überlagerten Emissionsregelung

geeigneter Identifikationssignale. Zum Beispiel wird der Parameter $k_{\mathrm{d}}$ aus der Sprungantwort des Katalysators ermittelt.

Regelung des Katalysators Einer der ersten Reglerentwürfe, bei dem die Katalysatordynamik berücksichtigt wird, wird von Roduner u. a. [85] durchgeführt. Roduner u. a. kompensieren den Offset der Breitband-Lambdasonde vor dem Katalysator durch einen PI-Regler und beziehen die als Integrator modellierte Sauerstoffspeicherdynamik beim Entwurf des Lambdaregelkreises ein. Angestrebt wird die Reduktion der Auswirkung der Lambdaabweichung im Motorabgas auf den Sauerstofffüllstand nur durch die Nutzung der Breitband-Lambdasonde vor dem Katalysator. Roduner $u$. a. entwerfen einen $\mathrm{H}_{\infty}$-Regler, welcher mittels Gain-Scheduling an den Motorarbeitspunkt angepasst wird. Der sehr ähnliche Entwurf von Shafai u. a. [90] unterscheidet sich darin, dass der Sensoroffset und die Integratorverstärkung $k_{\mathrm{I}}(t)$ in Gleichung (3.1) mittels einer rekursiven Markov-Schätzung fortlaufend angepasst werden. Für phänomenologische, eindimensionale Katalysatormodelle werden weiterhin P-Regler mit Gain-Scheduling, siehe die Arbeiten von Fiengo u. a. und Balenovic, oder modellprädiktive Regler wie die von Muske und Peyton Jones, Balenovic und Schallock u. a. entworfen. Um den Rechenaufwand dabei zu reduzieren, werden die modellprädiktiven Regler von Muske und Peyton Jones sowie Balenovic durch Radiale-BasisfunktionenNetze approximiert. Muske und Peyton Jones schlagen des Weiteren vor, den Sauerstoffspeicher gezielt zu befüllen oder zu entleeren und so das Motoransprechverhalten oder den Kraftstoffverbrauch zu verbessern.

Modellprädiktive Regler können das nichtlineare Verhalten des Katalysators berücksichtigen. Sie sind jedoch mit einem hohen Rechenaufwand verbunden, wenn ein in mehreren Zellen diskretisiertes Katalysatormodell verwendet wird. Aus diesem Grund werden für die in drei Zellen diskretisierten Modelle von Auckenthaler, Möller und Tomforde u. a. nach einer Linearisierung um den Arbeitspunkt LQ-Regler entworfen. Im Gütemaß des LQ-Reglers wird von Auckenthaler am stärksten die Abweichung des Sauerstofffüllstandes der zweiten Modellzelle vom Sollwert gewichtet, wogegen von Möller ausschließlich der dritte Sauerstofffüllstand gewichtet wird. Auckenthaler nimmt zusätzlich eine Erweiterung um einen I-Anteil vor, um die stationäre Genauigkeit des Sauerstofffüllstandes der dritten Modellzelle zu erreichen. Kiwic schlägt ebenfalls ein $\mathrm{Zu}-$ 
standsregler vor. Die Elemente der Reglerverstärkung werden dabei direkt so gewählt, dass sie linear über die Katalysatorlänge ansteigen.

Bekiaris-Liberis u. a. [9] setzen auch das in dieser Arbeit verwendete Katalysatormodell ein, allerdings wird der Entwurf des Beobachters und der Ausgangsrückführung auf Basis der partiellen Differentialgleichungen durchgeführt. Um dies zu ermöglichen, werden einige weitere Annahmen getroffen und das Modell weiter vereinfacht, siehe die Literaturübersicht am Anfang von Kapitel 3.

\subsection{Struktur des Regelungskonzeptes}

In Bild 6.1 ist das Strukturbild des in dieser Arbeit entwickelten Regelungskonzeptes für den Katalysator dargestellt, welches aus dem in Kapitel 2 beschriebenen Lambdaregelkreis und einer überlagerten Emissionsregelung besteht. Die Emissionsregelung umfasst ihrerseits einen OpenLoop-Beobachter und den Emissionsregler selbst.

In Abschnitt 2.2.3 wird gezeigt, dass die realisierbare Bandbreite des Lambdaregelkreises für alle Motorarbeitspunkte bei unter $1 \mathrm{~Hz}$ liegt. Vergleicht man diese Bandbreite mit den Zeitkonstanten des linearisierten Katalysatormodells in Abschnitt 4.3, so sieht man, dass bei mittleren Sauerstofffüllständen der Katalysator die Systemdynamik bestimmt. Bei niedrigen und hohen Sauerstofffüllständen hat dagegen der Lambdaregelkreis einen vergleichbaren Einfluss auf die Systemdynamik wie der Katalysator. Es ist einleuchtend, dass mit einer Verkleinerung der Sauerstoffspeicherfähigkeit des Katalysators der Sauerstofffüllstand öfters in die Randbereiche des Arbeitsbereichs gelangen wird. Daher sollte angestrebt werden, den Lambdaregelkreis beim Entwurf des überlagerten Emissionsregelkreises zu berücksichtigen.

Lambdaregelkreis Die Dynamik des geschlossenen Lambdaregelkreises bestimmt sich durch die Totzeit $\tau_{\mathrm{d}}$ und die Dynamik des IMC-Filters, das in Abschnitt 2.2.3 als eine Übertragungsfunktion zweiter Ordnung gewählt wird. Ohne Beschränkung der Allgemeinheit und um den weiteren Reglerentwurf zu vereinfachen, wird in diesem Kapitel der IMC-Lambdaregler zu $Q=1$ gewählt. Das heißt, der offene und der geschlossene Lambdaregelkreis besitzen die gleiche $\mathrm{PT}_{2}$ Dynamik, was in einer Verringerung der Bandbreite im Mittel über alle Motorarbeitspunkte um $15 \%$ und einer Erhöhung der Robustheit resultiert. Der Einfachheit halber wird im Folgenden die $\mathrm{PT}_{2}$-Dynamik des Lambdaregelkreises durch eine Übertragungsfunktion erster Ordnung, ein $\mathrm{PT}_{1}$-Glied mit der Zeitkonstante $\tau_{\text {imc }}$, approximiert, während die Totzeit unverändert bleibt. Des Weiteren wird der Lambdaregelkreis zeitkontinuierlich und nicht zeitdiskret betrachtet. Nach diesen Vereinfachungen gilt das Strukturbild in Bild 6.2 und für den Lambdawert im Motorabgas gilt die folgende Gleichung:

$$
\lambda_{\mathrm{m}}=\lambda_{\mathrm{d}}+\frac{1}{\tau_{\mathrm{imc}}(M, n) s+1} \mathrm{e}^{-\tau_{\mathrm{d}}(M, n) s}\left(\lambda_{\mathrm{w}}-\lambda_{\mathrm{d}}\right) .
$$




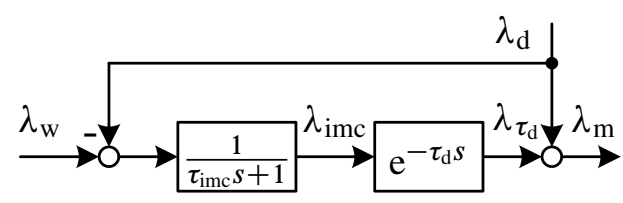

Bild 6.2: Äquivalentes Strukturbild des geschlossenen Lambdaregelkreises für den Entwurf der Emissionsregelung

Beobachter Die asymptotische Stabilität einerseits und die schlechte Beobachtbarkeit andererseits motivieren den Einsatz des Open-Loop-Beobachters. Die gestrichelte Linie vom Katalysatorausgang zum Beobachter in Bild 6.1 soll zum Ausdruck bringen, dass auf das Signal der SprungLambdasonde im überlagerten Emissionsregelkreis nicht komplett verzichtet wird. Zum einen wird das Signal der Sprung-Lambdasonde für die infolge der Alterung notwendige Parameteranpassung des Katalysatormodells verwendet und zum anderen kann in bestimmten Situationen der Open-Loop-Beobachter damit nachgeführt werden.

Auf die Parameteroptimierung während des Betriebs und auf mögliche Strategien zum Nachführen des Beobachters wird in dieser Arbeit nicht näher eingegangen. Das Lösen dieser beiden Aufgaben wird jedoch durch die Ergebnisse in Abschnitt 4.4 unterstützt. Zum Beispiel könnte die nichtlineare Beobachtbarkeitsmatrix in Gleichung (4.15) approximativ berechnet und die Beobachtbarkeit mit Hilfe der Konditionszahl der Matrix $\mathbf{M}_{\mathrm{b}}$ abgeschätzt werden. In den Situationen mit stärker ausgeprägter Beobachtbarkeit könnten dann die Signale der Lambdasonden zur Parameteroptimierung und zum Nachführen des Beobachters genutzt werden.

Emissionsregler Der Emissionsregler kann sowohl nichtlinear sein als auch auf der Linearisierung des Katalysatormodells basieren. Motiviert durch das stark nichtlineare Verhalten des Katalysators werden im Folgenden drei nichtlineare Emissionsregler entworfen. Zwei davon basieren auf exakter Ein-/Ausgangslinearisierung: Der erste Regler regelt den Sauerstofffüllstand im hinteren Bereich des Katalysators und der zweite die $\mathrm{O}_{2}$-Konvertierungsrate. Ein weiterer entworfener Regler ist ein LQ-Regler, dessen Gütemaß im Gegensatz zur Literatur modellbasiert parametriert wird. Anschließend wird mit demselben Gütemaß eine nichtlineare modellprädiktive Regelung entworfen. Während die ersten zwei genannten, nichtlinearen Regler den Lambdaregelkreis nur approximativ und der LQ-Regler diesen überhaupt nicht berücksichtigen, bezieht die modellprädiktive Regelung das komplette System sowie die Stellgrößenbeschränkung ein. Ein wichtiges Ziel der Arbeit ist es, die verschiedenen Konzepte für die Emissionsregler miteinander zu vergleichen.

\subsection{Regelung des Sauerstofffüllstandes}

Auckenthaler [4] beschreibt, dass die Gaskonzentrationen hinter dem Katalysator stark mit dem Sauerstofffüllstand im hinteren Bereich des Katalysators zusammenhängen. Möller [64] greift beim LQ-Reglerentwurf diese Idee ebenfalls auf und gewichtet nur den Sauerstofffüllstand der 

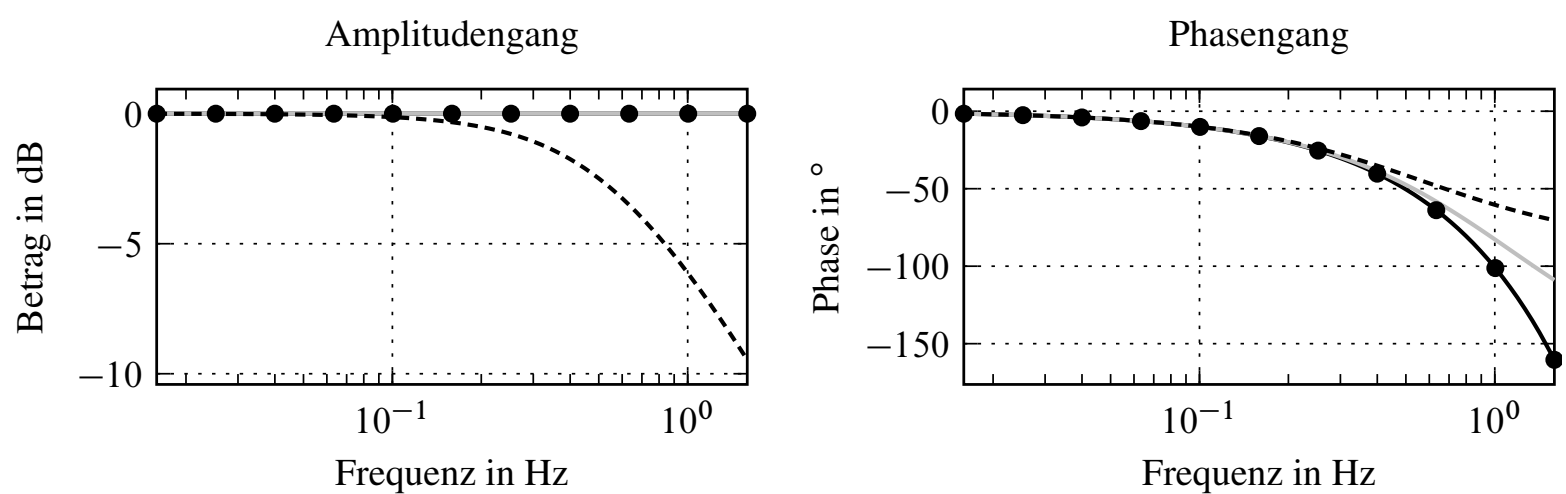

Bild 6.3: Vergleich des Totzeitgliedes ( $\bullet-)$ mit einer Padé-Approximation 1. Ordnung $(-)$ und einem $\mathrm{PT}_{1}$-Glied (----) für die maximale Totzeit des Motors von $0,28 \mathrm{~s}$

letzten Zelle des Katalysatormodells im quadratischen Gütemaß. Die Idee dahinter ist folgende: Die Lambdaabweichung im Motorabgas wirkt sich schnell auf den Sauerstofffüllstand im vorderen Bereich des Katalysators aus und wird entlang des Katalysators aufgrund seiner Sauerstoffspeicherfähigkeit gedämpft. Solange der Sauerstofffüllstand im hinteren Bereich des Katalysators einen mittleren Wert aufweist, können Lambdaabweichungen am Katalysatoreingang sowohl in magerer als auch in fetter Richtung gepuffert werden. Daher sind keine erhöhten Schadstoffemissionen hinter dem Katalysator zu erwarten. Motiviert durch das stark nichtlineare Verhalten des Katalysators wird im Folgenden ein Regler für den Sauerstofffüllstand der dritten Zelle des Katalysatormodells mittels exakter Ein-/Ausgangslinearisierung entworfen.

\subsubsection{Reglerentwurf mittels exakter Ein-/Ausgangslinearisierung}

Der Reglerentwurf mittels exakter Ein-/Ausgangslinearisierung besteht aus zwei Schritten: a) Systeminversion und damit Kompensation des nichtlinearen Systemverhaltens und b) Reglerentwurf für das im ersten Schritt entstandene lineare System [2, 45]. Die Systeminversion im ersten Schritt erfordert eine stabile Inverse, welche die nicht minimalphasige Totzeit in Gleichung (6.1) nicht besitzt. Die einfachste minimalphasige Approximation des Totzeitgliedes stellt ein $\mathrm{PT}_{1}$-Glied mit einer Zeitkonstante dar, die der Totzeit entspricht [32]. In Bild 6.3 ist ein Vergleich des Totzeitgliedes mit einem $\mathrm{PT}_{1}$-Glied und einer Padé-Approximation erster Ordnung dargestellt. Während beim Totzeitglied und der Padé-Approximation der Amplitudengang konstant bleibt, nimmt dieser des $\mathrm{PT}_{1}$-Glieds erwartungsgemäß mit der Frequenz ab. Bei der $3 \mathrm{~dB}$-Grenzfrequenz, die für die maximale Totzeit des Motors von $0,28 \mathrm{~s}$ bei etwa $0,57 \mathrm{~Hz}$ liegt, beträgt der relative Fehler für das $\mathrm{PT}_{1}$-Glied etwa $-30 \%$. An dieser Stelle liegt der relative Fehler des Phasengangs des $\mathrm{PT}_{1}$-Gliedes bei etwa $-21,5 \%$ und dieser der Padé-Approximation bei etwa $-7,3 \%$. Bedenkt man, dass die Totzeit hinter dem $\mathrm{PT}_{1}$-Glied in Bild 6.2 sitzt und die Zeitkonstante $\tau_{\text {imc }}$ des $\mathrm{PT}_{1}$-Gliedes ähnlich groß wie die Totzeit selbst ist, so sind die Signalfrequenzen am Eingang des Totzeitgliedes im Wesentlichen bereits auf die $3 \mathrm{~dB}-$ Grenzfrequenz beschränkt. ${ }^{1}$ Aus diesem Grund und da die größten Amplituden der Lambdaabweichung $\lambda_{\mathrm{d}}$ ohnehin im Intervall von 0 bis $0,5 \mathrm{~Hz}$ liegen und damit

\footnotetext{
${ }^{1}$ Dies ist ein weiterer Grund für die Wahl des IMC-Lambdareglers zu $Q=1$.
} 
die Komponenten im Lambdaregelkreis anregen, siehe Abschnitt 2.2.1, ist diese minimalphasige Approximation zulässig.

Systeminversion In [2, 45] wird bei der Durchführung der exakten Ein-/Ausgangslinearisierung von einem dynamischen System ausgegangen, das bezüglich der Eingangsgröße linear ist. Das Katalysatormodell selbst ist linear bezüglich der Eingangskonzentrationen $\mathbf{c}^{\text {in }}$, jedoch nicht bezüglich des Lambdawertes am Katalysatoreingang. Mit der $\mathrm{PT}_{1}$-Approximation des Totzeitgliedes und unter der Annahme einer abschnittsweisen konstanten Lambdaabweichung ergibt sich für ein Katalysatormodell mit drei Zellen das folgende Zustandsraummodell sechster Ordnung

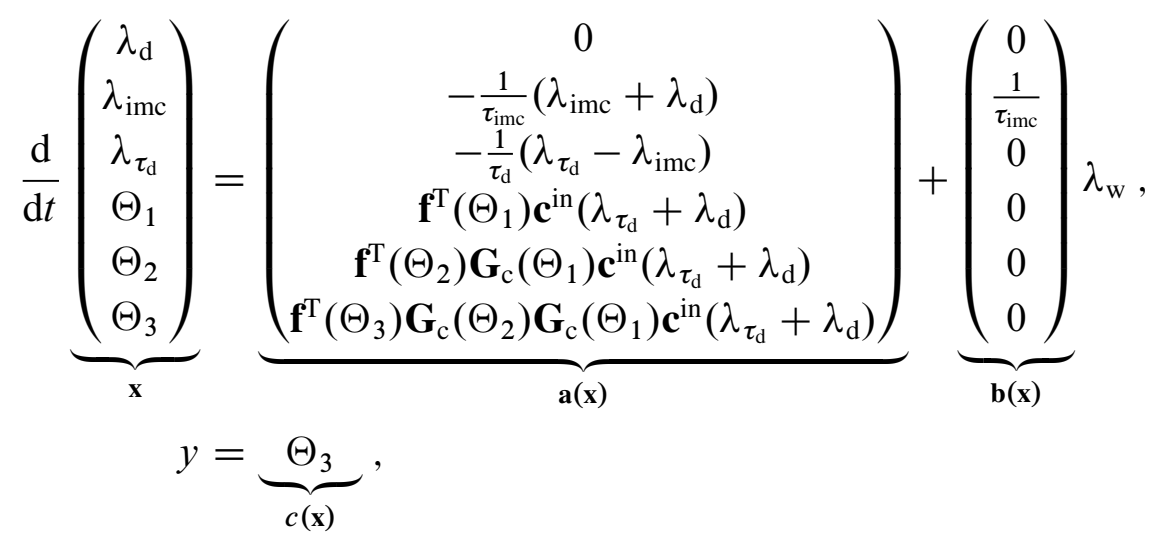

das linear bezüglich des Soll-Lambdawertes $\lambda_{\mathrm{w}}$ des Lambdaregelkreises ist. Damit liegt das Modell (6.2) in einer geeigneten Form für den Reglerentwurf vor. Die folgenden Ausführungen über den theoretischen Hintergrund der exakten Ein-/Ausgangslinearisierung sind an [2, 3, 45] angelehnt.

Die Differenzordnung $\delta$ eines Systems gibt an, wie oft die Ausgangsgröße $y$ abgeleitet werden muss, bevor die Eingangsgröße, hier $\lambda_{\mathrm{w}}$, in der Ableitung $y^{(\delta)}$ zum ersten Mal erscheint. In der geometrischen Regelungstheorie hat sich die Verwendung der sogenannten Lie-Ableitung bewährt. Sie ist als Gradient einer skalaren Funktion, z. B. $c(\mathbf{x})$, multipliziert mit einem Vektorfeld, z. B. a(x), definiert:

$$
L_{\mathbf{a}} c(\mathbf{x})=\frac{\partial c(\mathbf{x})}{\partial \mathbf{x}} \mathbf{a}(\mathbf{x}) .
$$

Mit den Lie-Ableitungen können die Ableitungen der Ausgangsgröße übersichtlich ausgedrückt werden und für die ersten drei Ableitungen von $y$ in Gleichung (6.2b) gilt:

$$
\begin{aligned}
& \dot{y}=L_{\mathbf{a}} c(\mathbf{x})+\underbrace{L_{\mathbf{b}} c(\mathbf{x})}_{=0} \lambda_{\mathrm{w}}, \\
& \ddot{y}=\underbrace{L_{\mathbf{a}} L_{\mathbf{a}} c(\mathbf{x})}_{L_{\mathbf{a}}^{2} c(\mathbf{x})}+\underbrace{L_{\mathbf{b}} L_{\mathbf{a}} c(\mathbf{x})}_{=0} \lambda_{\mathrm{w}}, \\
& \dddot{y}=L_{\mathbf{a}}^{3} c(\mathbf{x})+L_{\mathbf{b}} L_{\mathbf{a}}^{2} c(\mathbf{x}) \lambda_{\mathrm{w}} .
\end{aligned}
$$

Die Ausdrücke $L_{\mathbf{b}} c(\mathbf{x})$ und $L_{\mathbf{b}} L_{\mathbf{a}} c(\mathbf{x})$ in Gleichung (6.4a) und Gleichung (6.4b) sind identisch Null. Erst der Ausdruck $L_{\mathbf{b}} L_{\mathbf{a}}^{2} c(\mathbf{x})$ in der dritten Ableitung von $y$, Gleichung (6.4c), wird ungleich Null, was in einer Differenzordnung von drei resultiert. Diese Differenzordnung ist auch 


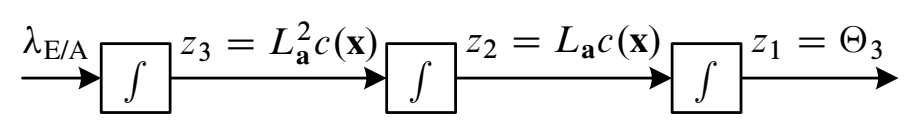

Bild 6.4: Zusammenhang zwischen den Zuständen der Integratorkette im transformierten Zustandsraummodell (6.7) und den Lie-Ableitungen (6.4)

intuitiv, denn der durch ein $\mathrm{PT}_{2}$-Glied approximierte Lambdaregelkreis besitzt eine Differenzordnung von zwei und der Sauerstofffüllstand jeder Zelle des Katalysatormodells hängt direkt vom Lambdawert am Katalysatoreingang ab. Das heißt, für $y=\Theta_{1 / 2 / \ldots / n_{\mathrm{c}}}$ besitzt das Katalysatormodell eine Differenzordnung von Eins. Da die Differenzordnung $\delta$ nicht der Systemordnung $n$ entspricht, hat das System eine interne Dynamik, die nach dem Reglerentwurf auf Stabilität untersucht werden sollte. Die Komplexität des Gesamtsystem erschwert jedoch diese Untersuchung, sodass in dieser Arbeit darauf verzichtet wird. Eine instabile interne Dynamik würde zu einem instabilen geschlossenen Regelkreis führen, siehe [2], wohingegen Simulationsstudien und die praktische Implementierung des Reglers am Laborprüfstand seine Stabilität belegen.

Durch die Lie-Ableitungen wird die nichtlineare Transformation

$$
\mathbf{z}=\mathbf{t}(\mathbf{x})
$$

definiert, wobei aufgrund der nicht vollen Differenzordnung $(\delta=3<n=6)$ die letzten drei Elemente der Vektorfunktion $\mathbf{t}$ beliebig gewählt werden können. Da die interne Dynamik nicht analytisch betrachtet wird, werden im vorliegenden Fall diese Elemente nicht explizit benötigt. Mit der nichtlinearen Transformation $\mathbf{t}$ wird das Zustandsraummodell (6.2) in die so genannte ByrnesIsidori-Normalform transformiert. Diese zeichnet sich dadurch aus, dass die Ausgangsgröße der ersten Zustandsgröße entspricht

$$
y=z_{1}
$$

und die ersten $\delta$ Zustände ausschließlich durch Integratoren verknüpft sind:

$$
\frac{\mathrm{d}}{\mathrm{d} t}\left(\begin{array}{c}
z_{1} \\
z_{2} \\
z_{3} \\
z_{4} \\
z_{5} \\
z_{6}
\end{array}\right)=\left(\begin{array}{c}
z_{2} \\
z_{3} \\
L_{\mathbf{a}}^{3} c(\mathbf{x})+L_{\mathbf{b}} L_{\mathbf{a}}^{2} c(\mathbf{x}) \lambda_{\mathrm{w}} \\
\dot{t}_{4}(\mathbf{x}) \\
\dot{t}_{5}(\mathbf{x}) \\
\dot{t}_{6}(\mathbf{x})
\end{array}\right) .
$$

Die Zustände $z_{4}, z_{5}$ und $z_{6}$ in Gleichung (6.7) haben keinen Einfluss auf die Ausgangsgröße und werden daher als interne Dynamik bezeichnet. Aus Gleichung (6.7) ist ersichtlich, dass das nichtlineare Ein-/Ausgangsverhalten durch folgende Wahl der Eingangsgröße kompensiert werden kann:

$$
\lambda_{\mathrm{w}}=\frac{\lambda_{\mathrm{E} / \mathrm{A}}-L_{\mathbf{a}}^{3} c(\mathbf{x})}{L_{\mathbf{b}} L_{\mathbf{a}}^{2} c(\mathbf{x})} .
$$

Hierbei bezeichnet $\lambda_{\mathrm{E} / \mathrm{A}}$ die neue Stellgröße, die am Eingang der Integratorkette mit den Zuständen $z_{1}, z_{2}$ und $z_{3}$ anliegt, siehe Bild 6.4. 
Differenzordnung Der Ausdruck $L_{\mathbf{b}} L_{\mathbf{a}}^{2} c(\mathbf{x})$ in Gleichung 6.4c ist zwar nicht identisch Null, jedoch ist er nicht im kompletten Arbeitsbereich auch ungleich Null, d. h. die Differenzordnung ist nicht im kompletten Arbeitsbereich wohldefiniert. Unabhängig vom Lambdawert am Katalysatoreingang oder den Sauerstofffüllständen der ersten zwei Modellzellen durchläuft die Lie-Ableitung bei sehr niedrigen Sauerstofffüllständen der dritten Modellzelle den Nullpunkt und wird negativ. Bei einer Diskretisierung des Katalysatormodells in drei Zellen ist dieses Verhalten unkritisch, da dieser Effekt sehr nahe am Rand des Arbeitsbereichs auftritt und der Katalysator in diesem Randbereich nicht gezielt betrieben wird. Wird jedoch das Katalysatormodell in vier oder mehr Zellen diskretisiert, so durchläuft die entsprechende Lie-Ableitung den Nullpunkt inmitten des Arbeitsbereichs. Aufgrund der Komplexität der Lie-Ableitungen kann die Ursache für dieses Verhalten nicht genauer untersucht und z. B. auf physikochemische Sachverhalte zurückgeführt werden. Eine Möglichkeit, mit der nicht wohldefinierten Differenzordnung umzugehen, stellen näherungsweise Verfahren zur Zustands- und zur Ein-/Ausgangslinearisierung dar [3]. Zum Beispiel wird bei der näherungsweisen Ein-/Ausgangslinearisierung zu der Lie-Ableitung $L_{\mathbf{b}} L_{\mathbf{a}}^{2} c(\mathbf{x})$ die Störfunktion $\epsilon(\mathbf{x})$ addiert und diese so gewählt, dass eine Singularität vermieden wird. Eine weitere Möglichkeit stellt die Poincaré-Linearisierung dar, bei der nicht alle Systemnichtlinearitäten, sondern nur die quadratischen und eventuell die kubischen Terme nach einer Taylor-Reihenentwicklung kompensiert werden.

Regelung der Integratorkette Im zweiten Schritt des Reglerentwurfs wird ein Zustandsregler und ein Vorfilter für die im ersten Schritt entstandene Integratorkette dritter Ordnung entworfen, siehe Bild 6.4. Dabei werden die drei Pole an die gleiche Stelle gelegt, sodass diese Pollage als der einzige einzustellende Parameter des Reglerentwurfs verbleibt. Die Implementierung des nichtlinearen Reglers erfordert die Kenntnis der sechs Zustände des Modells in Gleichung (6.2a). Schätzungen der Zustände $\lambda_{\mathrm{d}}, \lambda_{\text {imc }}$ und $\lambda_{\tau_{\mathrm{d}}}$ werden vom internen Modell des IMC-Lambdaregelkreises geliefert und der Open-Loop-Beobachter in Bild 6.1 liefert eine Schätzung der drei Sauerstofffüllstände.

Simulationen Im Folgenden wird der mittels exakter Ein-/Ausgangslinearisierung entworfene Regler mit einer Lambda-Eins-Regelstrategie anhand einer Simulation verglichen. Wie bereits am Anfang dieses Kapitels erläutert, wird bei einer Lambda-Eins-Regelstrategie ausschließlich der Lambdawert $\lambda_{\mathrm{m}}$ vor dem Katalysator geregelt. In dieser Arbeit repräsentiert diese Regelstrategie den Stand der Technik. In einem zweiten Vergleich wird das prinzipielle Verhalten des Reglers an verschiedenen Motorarbeitspunkten diskutiert.

In Bild 6.5 wird der mittels exakter Ein-/Ausgangslineariserung entworfene Regler mit einer Lambda-Eins-Regelstrategie verglichen. Der zweite Motorarbeitspunkt wird gewählt, siehe Anhang A.1, und der dreifache Pol der Integratorkette auf -1,4 gelegt. In Bild 6.5 sind die Verläufe einer synthetischen Lambdaabweichung $\lambda_{\mathrm{d}}$, des Sollwertes $\lambda_{\mathrm{w}}$ des Lambdaregelkreises und der Sauerstofffüllstände der drei Modellzellen dargestellt. Die sprungförmige Lambdaabweichung stellt eine besonders starke Anforderung an die Regler dar und eignet sich gut für ihre theoretische Verifikation. Die Sprungamplitude beträgt beim ersten Sprung 1,25 \% und steigt um denselben Betrag bei jedem weiteren Sprung an. Die Sprunghöhe bei 160 s beträgt 8,75 \% und deckt damit einen 

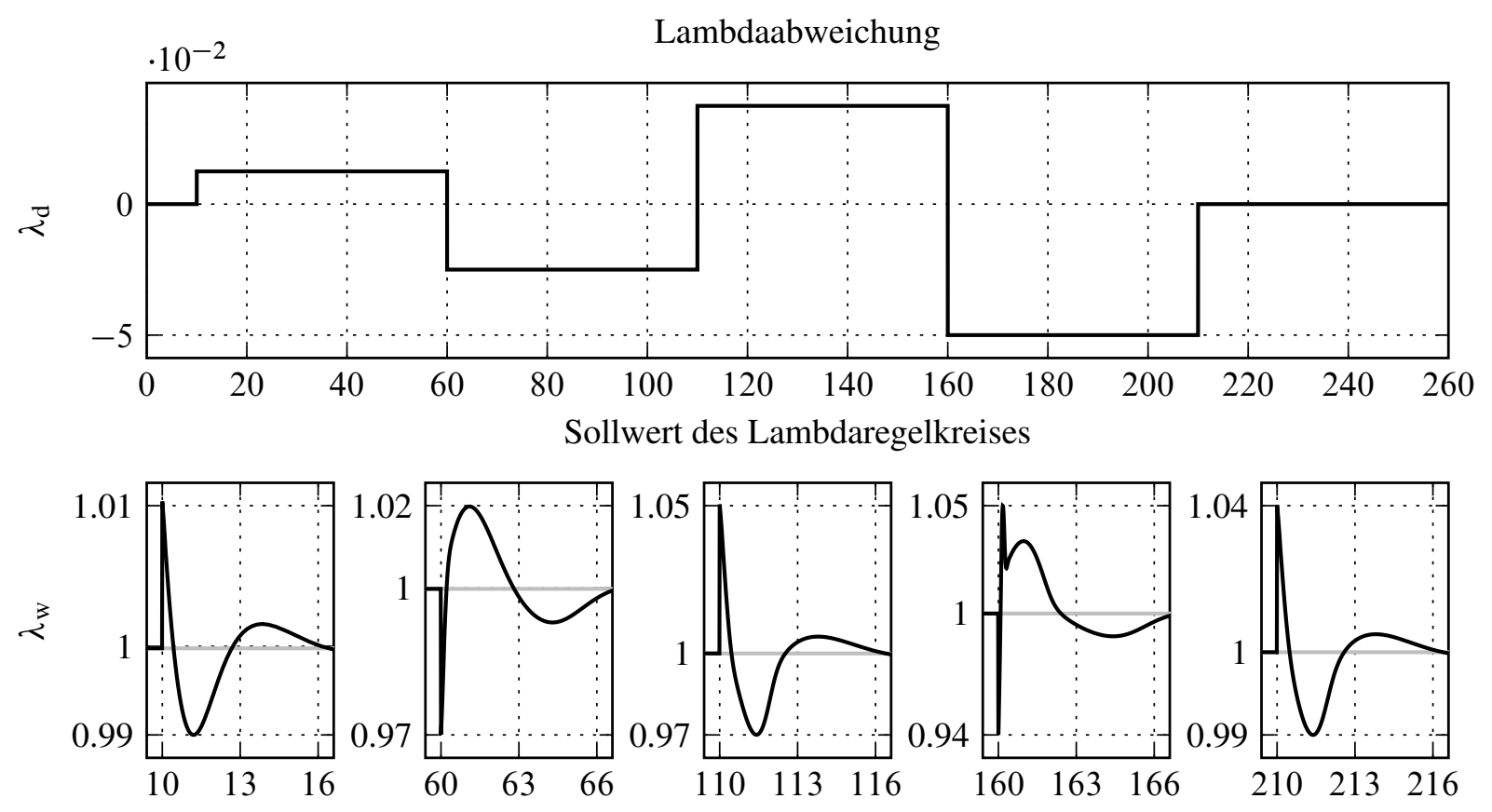

Sauerstofffüllstände der drei Modellzellen
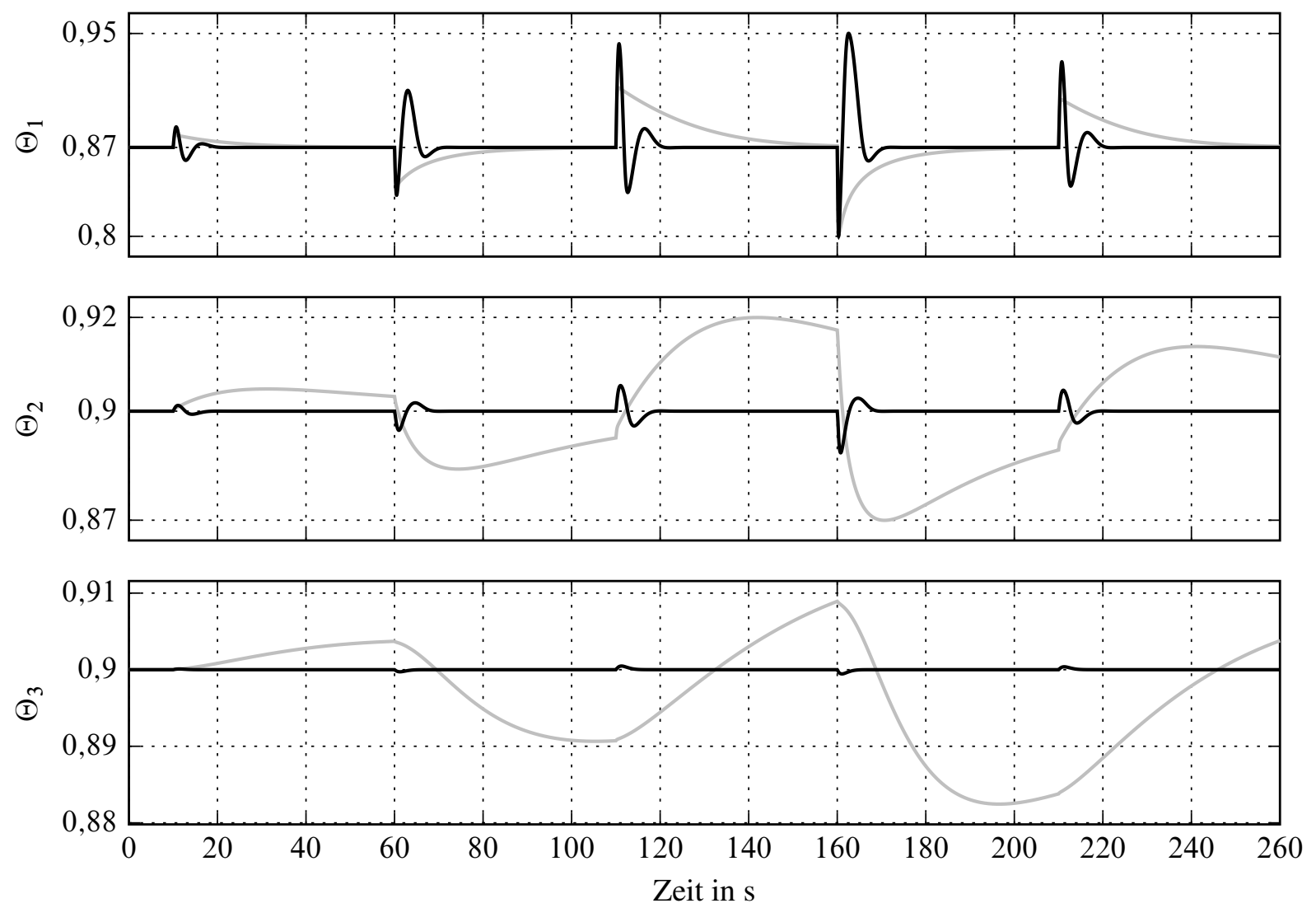

Bild 6.5: Vergleich des mittels exakter Ein-/Ausgangslinearisierung entworfenen Reglers (-) mit einer Lambda-Eins-Regelstrategie (-). Es wird der zweite Motorarbeitspunkt gewählt, siehe Anhang A.1, und der dreifache Pol der Integratorkette auf -1,4 gelegt. 
großen Arbeitsbereich ab. Da die Lambda-Eins-Regelstrategie das dynamische Verhalten des Katalysators nicht berücksichtigt, kommt es zu großen Abweichungen der Sauerstofffüllstände von den Sollwerten. An den Verläufen ist gut zu erkennen, dass aufgrund der asymptotischen Stabilität des Arbeitspunktes die Sauerstofffüllstände nach dem Ausregeln der Lambdaabweichung wieder in Richtung des Arbeitspunktes verlaufen. Die Maxima der Zeitkonstanten liegen für den gewählten Motorarbeitspunkt oberhalb des Arbeitspunktes, siehe Bild 4.6a, und so kommt es dazu, dass die Abweichungen in Richtung niedriger Sauerstofffüllstände schneller abnehmen als diese in Richtung höherer Sauerstofffüllstände. Aufgrund der sehr großen Zeitkonstanten der hinteren Modellzellen benötigen ihre Sauerstofffüllstände jedoch mehrere hundert Sekunden, um den Sollwert zu erreichen. Trotz der langsamen Dynamik sind die absoluten Abweichungen der Sauerstofffüllstände von den Sollwerten aber relativ klein. Deshalb kann die Lambda-Eins-Regelstrategie bei größeren Katalysatoren zufriedenstellend funktionieren. Die Regelung mittels exakter Ein/Ausgangslinearisierung weist dagegen eine sehr hohe Regelgüte auf und kann den Sauerstofffüllstand der dritten Modellzelle praktisch konstant auf dem Sollwert halten. Am Verlauf des Sauerstofffüllstandes der ersten Modellzelle ist gut zu erkennen, dass dieser nach dem Sprung der Lambdaabweichung in die entgegengesetzte Richtung geführt wird, um den Einfluss der Lambdaabweichung auf die hinteren Modellzellen zu reduzieren.

In Bild 6.6 sind drei Simulationen des mittels exakter Ein-/Ausgangslinearisierung entworfenen Reglers für eine Auslegung an den drei Motorarbeitspunkten in Anhang A.1 dargestellt. Am ersten Motorarbeitspunkt wird der dreifache Pol der Integratorkette auf -0,2, am zweiten Motorarbeitspunkt wie weiter oben auf -1,4 und am dritten Motorarbeitspunkt auf -9,5 gelegt. Die Sollwerte für den Sauerstofffüllstand werden wie in Abschnitt 5.3 durch eine Mittelung der Sauerstofffüllstände der drei Modellzellen im Lambdabereich von 0,98 bis 1,02 festgelegt. Interessanterweise liegt der so ermittelte Sauerstofffüllstand der dritten Modellzelle bei allen Arbeitspunkten bei etwa 0,9. Um die Darstellung der Ergebnisse im weiteren Verlauf der Arbeit zu erleichtern, wird der Arbeitspunkt so gewählt, dass die dritte Modellzelle einen Füllstand von genau 0,9 aufweist. Das Ziel bei der Reglerauslegung ist es, die Regler möglichst schnell auszulegen und gleichzeitig die Stellgrößenausnutzung auf $\pm 10 \%$ zu begrenzen. Mit steigender Motorarbeitspunktnummer steigen die Abgastemperatur und der Abgasmassenstrom, sodass die Zeitkonstanten des Katalysators, siehe Abschnitt 4.5, sowie die Zeitkonstante und die Totzeit im Lambdaregelkreis sinken. Dies führt dazu, dass die Regelgüte am dritten Motorarbeitspunkt am besten und gleichzeitig die Stellgrößenausnutzung am geringsten ist. Die Stellgrößenausnutzung am zweiten Motorarbeitspunkt nimmt im Vergleich zu der am dritten Motorarbeitspunkt leicht zu, während die Regelgüte im Vergleich dazu leicht abnimmt. Die Abweichungen des Sauerstofffüllstandes der dritten Modellzelle vom Sollwert sind aber immer noch sehr klein. Für den ersten Motorarbeitspunkt kann dagegen keine Auslegung gefunden werden, sodass die Stellgrößenbeschränkung nicht verletzt wird. Der Stellgrößenverlauf weist kleine Schwingungen auf und die großen Abweichungen der Sauerstofffüllstände von den Sollwerten werden nur sehr langsam ausgeregelt. Das stark ausgeprägt nichtlineare Katalysatorverhalten am ersten Motorarbeitspunkt kann zwar grundsätzlich mit einem höheren Stellaufwand einhergehen, jedoch liegt die Ursache für dieses Verhalten in der PT 1 -Approximation des Totzeitgliedes. Die Fouriertransformierte eines Sprungs enthält alle Frequenzen, die minimalphasige Approximation des Totzeitgliedes ist dagegen nur bis etwa zur 

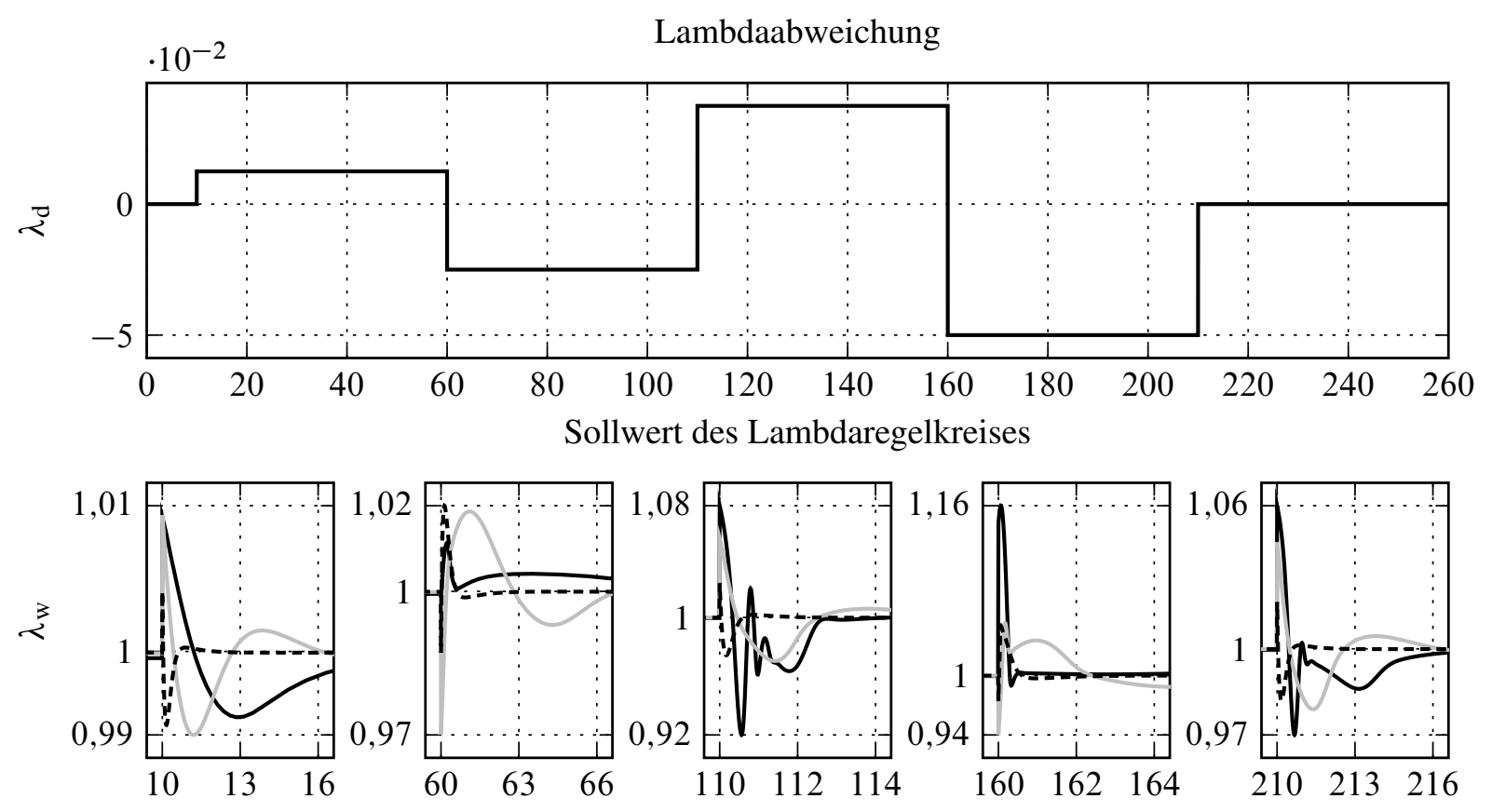

Sauerstofffüllstände der drei Modellzellen
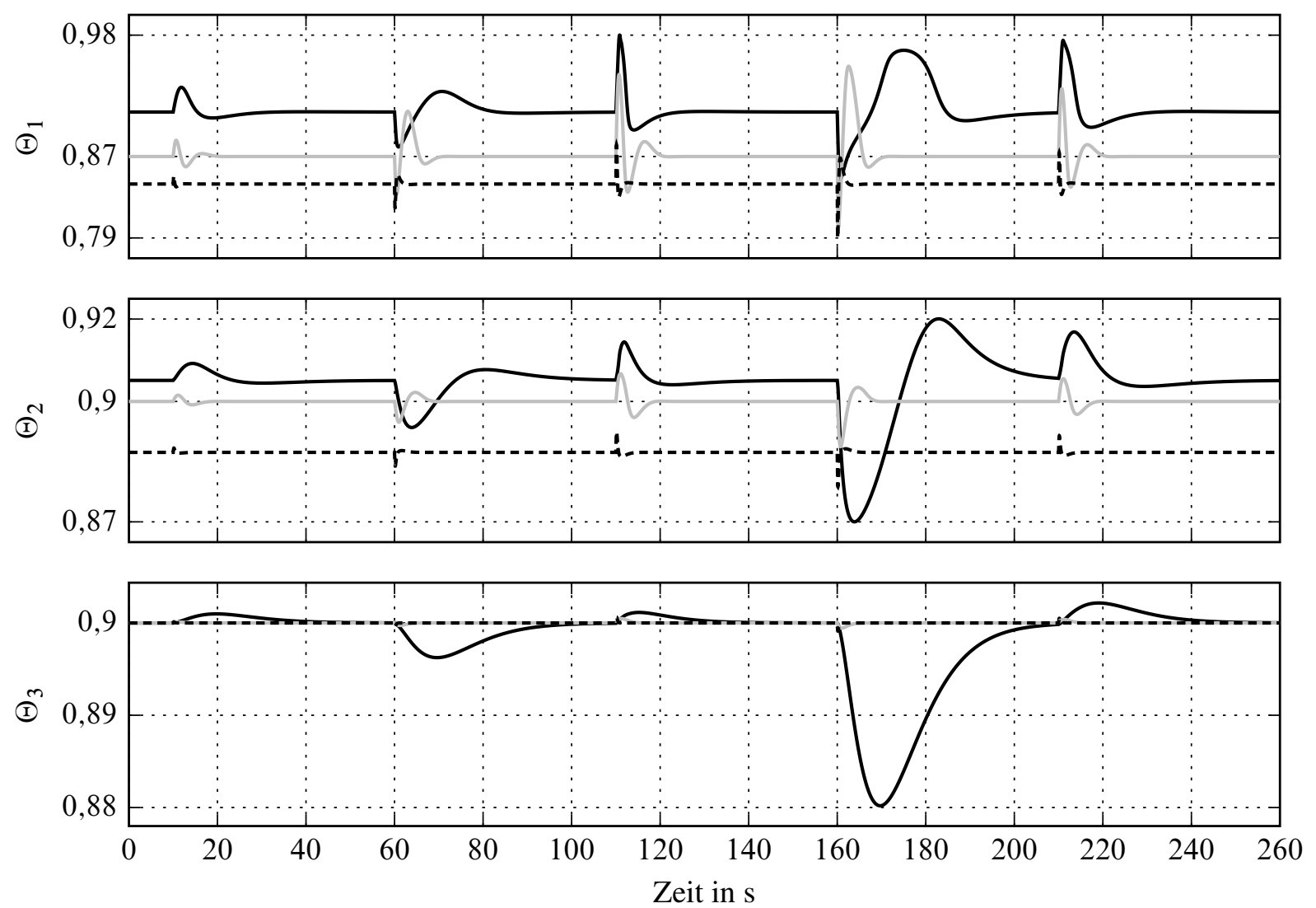

Bild 6.6: Vergleich des mittels exakter Ein-/Ausgangslinearisierung entworfenen Reglers für eine Auslegung für die drei Motorarbeitspunkte, siehe Anhang A.1. Der dreifache Pol liegt am ersten Motorarbeitspunkt (-) auf $-0,2$, am zweiten Motorarbeitspunkt ( - ) auf $-1,4$ und am dritten Motorarbeitspunkt (----) auf -9,5. 
$3 \mathrm{~dB}-$ Grenzfrequenz gültig. Da für große Zeitkonstanten und Totzeiten im Lambdaregelkreis die $3 \mathrm{~dB}$-Grenzfrequenz sinkt, wird auch die Approximation des Totzeitgliedes schlechter. Motoren mit Direkteinspritzung weisen kleinere Totzeiten als Motoren mit Saugrohreinspritzung auf, da der Kraftstoff nicht am Anfang des Einsaugtaktes, sondern während des Verdichtungstaktes eingespritzt wird. Wird der verwendete Motor gedanklich mit Direkteinspritzung ausgestattet und dieselbe Simulation mit einer um ein Drittel kleineren Totzeit durchgeführt, so nimmt die Stellgrößenausnutzung stark ab und die Schwingungen sind nicht mehr vorhanden, siehe Bild 6.7.

Zusammenfassung Nach einer minimalphasigen Approximation des Totzeitgliedes im Motormodell kann ein Regler für den Sauerstofffüllstand der dritten Zelle des Katalysatormodells mittels exakter Ein-/Ausgangslinearisierung entworfen werden. Verglichen mit einer Lambda-EinsRegelstrategie kann der nichtlineare Regler den Sauerstofffüllstand sehr gut regeln. Die minimalphasige Approximation der Totzeit erreicht jedoch bei großen Totzeiten im Lambdaregelkreis, z. B. bei niedrigen Motordrehzahlen, ihre Grenzen und sorgt für Schwingungen der Stellgröße. Unabhängig davon sorgt die Kompensation der kompletten, stark nichtlinearen Systemdynamik für einen hohen Stellaufwand und damit für große Abweichungen der Sauerstofffüllstände der ersten Zellen von ihren Sollwerten. Im nächsten Abschnitt wird untersucht, inwieweit dies eine Auswirkung auf die Umsetzung der Schadstoffe hat.

Der Reglerentwurf mittels Systeminversion ist auf sehr genaue Systemmodelle angewiesen und darunter kann in der Praxis die Robustheit des Regelkreises leiden. Aufgrund des Open-LoopBeobachters handelt es sich beim Emissionsregler jedoch streng genommen um eine Steuerung, sodass Diskrepanzen zwischen dem realen Katalysator und dem Katalysatormodell keine Rolle spielen. Einzig die Diskrepanz zwischen dem Totzeitglied im Motormodell des IMC-Lambdaregelkreises und der entsprechenden Approximation im Modell (6.2) für den Entwurf des Emissionsreglers führt zu der oben beschriebenen Problematik.

\subsection{Regelung der Konvertierungsraten}

Die im vorherigen Abschnitt vorgestellte Regelung des Sauerstofffüllstandes einer Modellzelle berücksichtigt nur einen Teil des Katalysators. Des Weiteren wird die Konvertierung der Schadstoffe im Katalysator nicht explizit berücksichtigt. Andererseits ist die Intention beim Einsatz des Katalysators gerade die Maximierung der Schadstoffkonvertierung. Der Grad der Konvertierung einer Gasspezies $i$ wird durch die Konvertierungsrate $\eta_{i}$ beschrieben und diese ist wie folgt definiert:

$$
\eta_{i}=1-\frac{c_{i}^{\text {out }}}{c_{i}^{\text {in }}} .
$$

Hierbei bezeichnet $c_{i}^{\text {in }}$ die Konzentration der Gasspezies $i$ am Katalysatoreingang und $c_{i}^{\text {out }}$ die entsprechende Konzentration am Katalysatorausgang. Da das in Abschnitt 3.1.2 vorgestellte Reaktionsschema nicht alle Schadstoffe im Motorabgas enthält, werden die NO- und die $\mathrm{C}_{x} \mathrm{H}_{y^{-}}$ Konzentration in Abschnitt 3.1.4 in äquivalente $\mathrm{O}_{2-}$, CO- oder $\mathrm{H}_{2}$-Konzentrationen umgerechnet. 

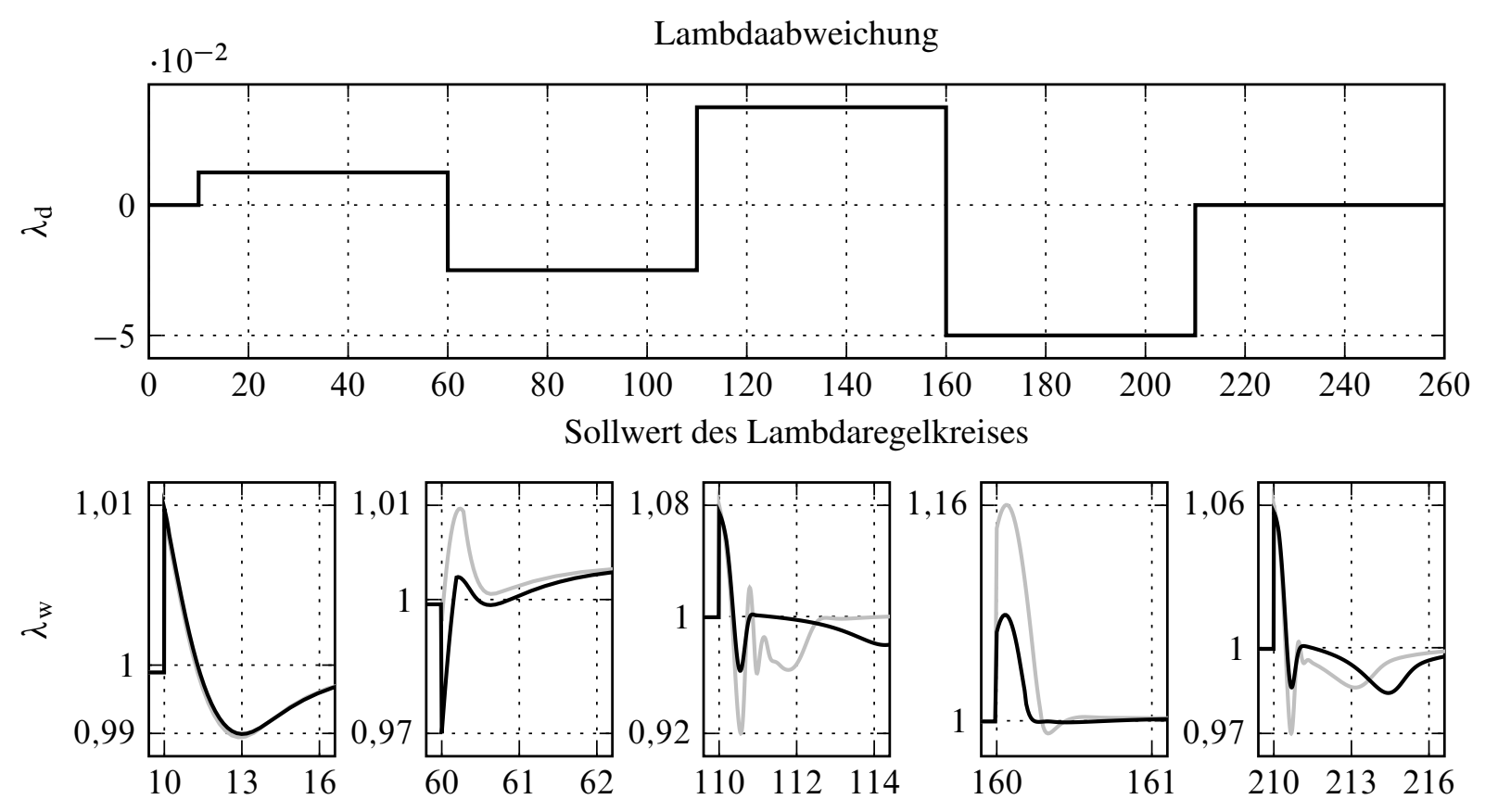

Sauerstofffüllstände der drei Modellzellen
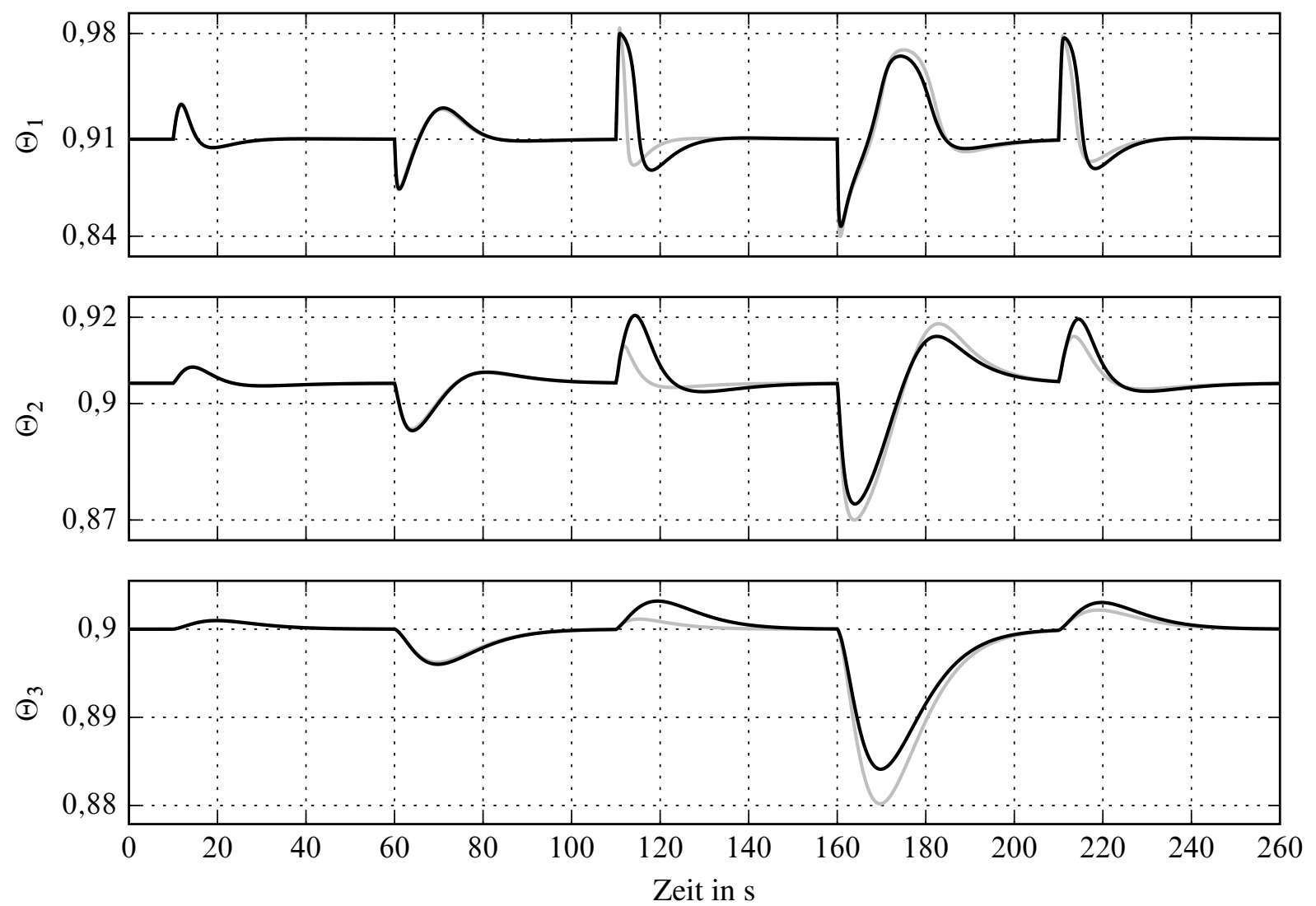

Bild 6.7: Vergleich des mittels exakter Ein-/Ausgangslinearisierung entworfenen Reglers für eine Auslegung am ersten Motorarbeitspunkt bei einer Variation der Totzeit des Motors: $\tau_{\mathrm{d}}$ $(-), 2 / 3 \tau_{\mathrm{d}}(-)$. Der dreifache Pol liegt jeweils auf $-0,2$. 


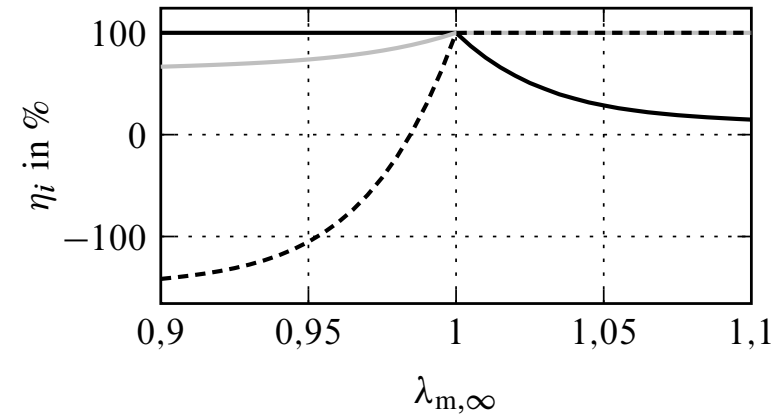

Bild 6.8: Verlauf der stationären Konvertierungsraten von $\mathrm{O}_{2}(-), \mathrm{CO}$ (-) und $\mathrm{H}_{2}(----)$

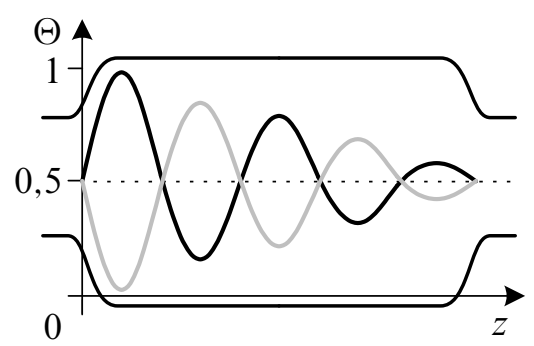

Bild 6.9: Auswirkung der Lambdaabweichung am Katalysatoreingang auf den Sauerstofffüllstand

Damit kann als neues Regelziel die Maximierung der Konvertierungsraten dieser drei Gasspezies definiert werden.

Die Konzentration $c_{i}^{\text {out }}$ am Ausgang des Katalysatormodells kann leicht analytisch berechnet werden. Beispielsweise ergibt sich für die CO-Konvertierungsrate der folgende Ausdruck

$$
\begin{aligned}
\eta_{\mathrm{CO}} & =1-\frac{\mathbf{I}_{\mathrm{CO}}^{\mathrm{T}} \mathbf{G}_{\mathrm{c}}\left(\Theta_{3}\right) \mathbf{G}_{\mathrm{c}}\left(\Theta_{2}\right) \mathbf{G}_{\mathrm{c}}\left(\Theta_{1}\right) \mathbf{c}^{\mathrm{in}}\left(\lambda_{\mathrm{m}}\right)}{c_{\mathrm{CO}}^{\mathrm{in}}} \\
& =1-\mathbf{I}_{\mathrm{CO}}^{\mathrm{T}} \mathbf{G}_{\mathrm{c}}\left(\Theta_{3}\right) \mathbf{G}_{\mathrm{c}}\left(\Theta_{2}\right) \mathbf{G}_{\mathrm{c}}\left(\Theta_{1}\right)\left(\begin{array}{c}
\frac{c_{\mathrm{CO} 2}^{\mathrm{in}}\left(\lambda_{\mathrm{m}}\right)}{c_{\mathrm{CO}}\left(\lambda_{\mathrm{m}}\right)} \\
0 \\
0 \\
1 \\
0
\end{array}\right)
\end{aligned}
$$

mit dem Vektor $\mathbf{I}_{\mathrm{CO}}^{\mathrm{T}}=\left(\begin{array}{lllll}0 & 0 & 0 & 1 & 0\end{array}\right)$. Aufgrund der reversiblen Reaktionen in den Reaktionsgleichungen (R1) und (R2) hängen die CO- und die $\mathrm{H}_{2}$-Konvertierungsrate auch von der $\mathrm{CO}_{2}$ - und der $\mathrm{H}_{2} \mathrm{O}$-Eingangskonzentration und dadurch auch explizit vom Lambdawert $\lambda_{\mathrm{m}}$ ab. Die $\mathrm{O}_{2}-\mathrm{Kon}$ vertierungsrate hängt dagegen nur von den Sauerstofffüllständen der drei Modellzellen ab. An den stationären Arbeitspunkten ausgewertet, sind die Konvertierungsraten monotone Funktionen von $\lambda_{\mathrm{m}, \infty}$, siehe Bild 6.8. Dabei begünstigen hohe Lambdawerte bzw. hohe Sauerstofffüllstände die $\mathrm{CO}$ - und die $\mathrm{H}_{2}$-Konvertierung und niedrige Lambdawerte bzw. niedrige Sauerstofffüllstände die $\mathrm{O}_{2}$-Konvertierung. In Bild 6.8 ist zu sehen, dass die $\mathrm{H}_{2}$-Konvertierungsrate auch negativ werden kann, d. h. durch die Rückwärtsreaktion der Reaktion (R2) wird aus dem Wasser im Motorabgas Wasserstoff generiert.

Wahl des Arbeitspunktes Die Verläufe der Konvertierungsraten können dazu genutzt werden, den stationären Arbeitspunkt zu finden, der zu einer möglichst hohen Schadstoffkonvertierung führt. Dafür werden die $\mathrm{O}_{2-}$, die $\mathrm{CO}$ - und die $\mathrm{H}_{2}$-Konvertierungsrate gewichtet miteinander addiert:

$$
\eta_{\Sigma}=w_{\mathrm{O}_{2}} \eta_{\mathrm{O}_{2}}+w_{\mathrm{CO}} \eta_{\mathrm{CO}}+w_{\mathrm{H}_{2}} \eta_{\mathrm{H}_{2}}
$$


Die Stelle, an der $\eta_{\Sigma}$ sein Maximum annimmt, bestimmt den Arbeitspunkt, d. h. den Lambdawert $\lambda_{\mathrm{m}, \infty}$ und den Sauerstofffüllstand entlang des Katalysators. Die Werte der Gewichte $w_{i}$ in Gleichung (6.11) liegen im geschlossenen Intervall [0,1]. Ihre Wahl spiegelt die Intention des Entwicklers wider, eine Gasspezies schwächer oder stärker zu berücksichtigen. Auckenthaler [4] bestimmt den Arbeitspunkt auf eine ähnliche Weise, allerdings nur anhand einer Betrachtung der letzten Zelle des von ihm eingesetzten Katalysatormodells.

Diese Wahl des Arbeitspunktes berücksichtigt jedoch nicht den transienten Motor- und Katalysatorbetrieb. Die Lambdaabweichung $\lambda_{d}$ wirkt sich schnell auf den Sauerstofffüllstand im vorderen Bereich des Katalysators aus. Der Arbeitspunkt sollte vorzugsweise so gewählt werden, dass im normalen Betrieb der Sauerstoffspeicher nicht komplett befüllt oder komplett entleert wird. Da man aufgrund der Ressourceneffizienz den Katalysator möglichst klein auslegen wird, wandert der Arbeitspunkt in die Mitte des Arbeitsbereichs. Damit nimmt die praktische Relevanz von Gleichung (6.11) ab. In Bild 6.9 ist diese Überlegung grafisch illustriert.

In den folgenden Abschnitten werden Emissionsregler vorgestellt, bei deren Auslegung oder Entwurf die Konvertierungsraten der Gasspezies berücksichtigt werden.

\subsubsection{LQ-Reglerentwurf}

Obwohl der Katalysator ein stark nichtlineares System darstellt, werden in der Literatur oft LQRegler für die Regelung von seinem Sauerstofffüllstand eingesetzt, siehe den Literaturüberblick am Anfang dieses Kapitels. Beim LQ-Reglerentwurf für den Katalysator wird das folgende quadratische Gütemaß

$$
J=\int_{0}^{\infty} \Delta \boldsymbol{\Theta}^{\mathrm{T}} \mathbf{Q} \Delta \boldsymbol{\Theta}+R \Delta \lambda_{\mathrm{m}}^{2} \mathrm{~d} t
$$

minimiert. Die Matrix $\mathbf{Q}$ gewichtet hierbei die Abweichungen $\Delta \boldsymbol{\Theta}$ der Sauerstofffüllstände der diskreten Modellzellen von ihren Sollwerten $\boldsymbol{\Theta}_{\mathrm{w}}$ und der Faktor $R$ gewichtet den Stellaufwand [60]. $\Delta \lambda_{\mathrm{m}}$ entspricht der Differenz von $\lambda_{\mathrm{m}}$ und dem Lambdawert $\lambda_{\mathrm{m}, \infty}$ am gewählten Arbeitspunkt.

Auckenthaler [4], Möller [64] und Tomforde u. a. [102] vernachlässigen die Dynamik des unterlagerten Lambdaregelkreises und deshalb kommen in Gleichung (6.12) die Zustände des Lambdaregelkreises oder sein Sollwert $\lambda_{w}$ nicht vor. Bedenkt man, dass der LQ-Reglerentwurf auf einer Linearisierung des Katalysatormodells um einen mittleren Sauerstofffüllstand basiert und dass für mittlere Sauerstofffüllstände die Systemdynamik vom Katalysator dominiert wird, so ist diese Annahme zulässig. Daher wird der Lambdaregelkreis in diesem Abschnitt ebenfalls als ideal angenommen und der LQ-Regler durch Minimierung des Gütemaßes in Gleichung (6.12) entworfen. Diese Vereinfachung ermöglicht nicht zuletzt einen Vergleich zu den bereits existierenden Reglerentwürfen von Auckenthaler und Möller. Tomforde u. a. gehen nicht auf die Wahl der Gewichtungsmatrix $\mathbf{Q}$ ein. Auckenthaler gewichtet mit der Matrix $\mathbf{Q}$ den Sauerstofffüllstand der zweiten Modellzelle und der der dritten Modellzelle gewichtet er um den Faktor 100 schwächer. Möller gewichtet dagegen ausschließlich den Sauerstofffüllstand der dritten Modellzelle. Das heißt, in der 


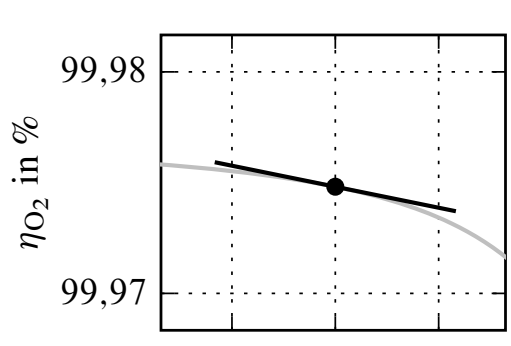

$\mathrm{O}_{2}$-Konvertierungsrate
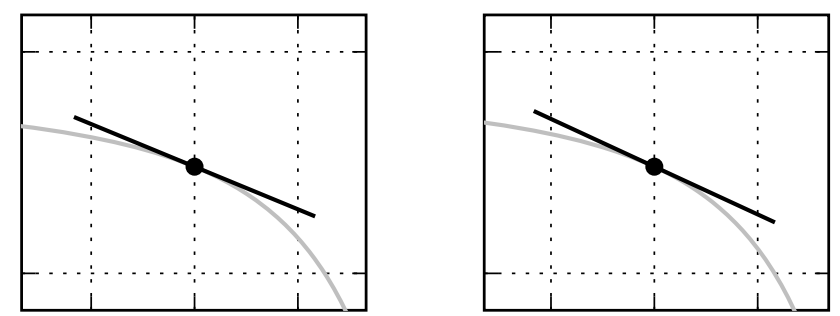

CO-Konvertierungsrate
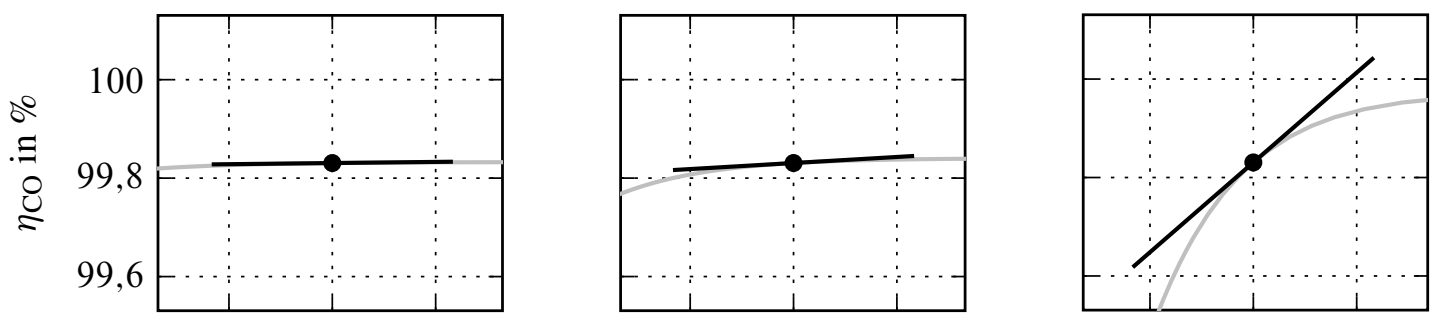

$\mathrm{H}_{2}$-Konvertierungsrate
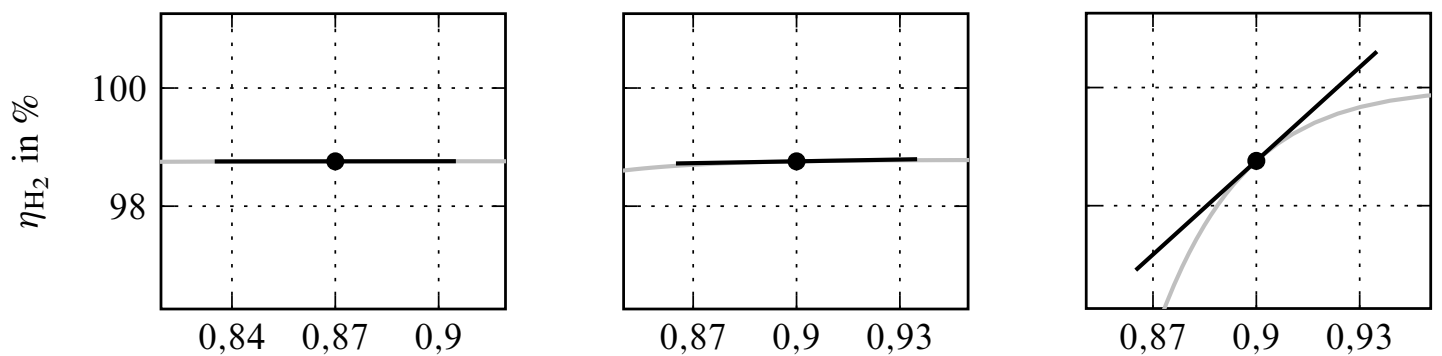

$\Theta_{1}$

$\Theta_{2}$

$\Theta_{3}$

Bild 6.10: Sensitivität der $\mathrm{O}_{2}$-, der $\mathrm{CO}$ - und der $\mathrm{H}_{2}$-Konvertierungsrate auf Änderungen der Sauerstofffüllstände der drei Modellzellen: Konvertierungsrate (-), Gradient am Arbeitspunkt $(\rightarrow-)$

Literatur erfolgt die Wahl von $\mathbf{Q}$ nicht konsistent und anhand qualitativer Kriterien. Im Folgenden wird dagegen eine modellbasierte Wahl dieser Gewichtungsmatrix vorgeschlagen.

Modellbasierte Wahl von $\mathbf{Q}$ Den Ausgangspunkt der modellbasierten Wahl von $\mathbf{Q}$ stellen die $\mathrm{O}_{2^{-}}$, die $\mathrm{CO}$ - und die $\mathrm{H}_{2}$-Konvertierungsrate dar. Es liegt nahe, die Modellzellen beim LQ-Reglerentwurf nach ihrer Wichtigkeit für die Konvertierung dieser Gasspezies zu gewichten. Durch das Ableiten der Konvertierungsraten nach den einzelnen Sauerstofffüllständen, d. h. durch die Bildung des Gradienten, kann die Sensitivität der Konvertierungsraten im Hinblick auf die einzelnen Sauerstofffüllstände entlang des Katalysators berechnet werden:

$$
S_{\eta_{i}}=\operatorname{grad}\left(\eta_{i}\right)=\left(\begin{array}{lll}
\frac{\partial \eta_{i}}{\partial \Theta_{1}} & \frac{\partial \eta_{i}}{\partial \Theta_{2}} & \frac{\partial \eta_{i}}{\partial \Theta_{3}}
\end{array}\right)^{\mathrm{T}} \text { mit } i \in\left\{\mathrm{O}_{2}, \mathrm{CO}, \mathrm{H}_{2}\right\}
$$

In Bild 6.10 sind die Verläufe der drei Konvertierungsraten in Abhängigkeit der Sauerstofffüllstände der drei Modellzellen sowie die Tangenten am gewählten Arbeitspunkt dargestellt. Die 
$\mathrm{CO}$ - und die $\mathrm{H}_{2}$-Konvertierungsrate verhalten sich ähnlich und hängen vor allem vom Sauerstofffüllstand der dritten Modellzelle ab, während die $\mathrm{O}_{2}$-Konvertierungsrate vom Sauerstoffüllstand entlang des kompletten Katalysators abhängt. Diese Analyse zeigt, dass Möller [64] mit der Wahl der Gewichtungsmatrix auf eine Minimierung der $\mathrm{CO}$ - und der $\mathrm{H}_{2}$-Emissionen abzielt.

Aus den Sensitivitäten in Gleichung (6.13) kann für jede der drei Gasspezies eine diagonale Gewichtungsmatrix $\mathbf{Q}_{i}$ erstellt werden. Da bei der Gewichtung der Zustände im Gütemaß (6.12) nur die Verhältnisse der Gewichte zueinander, jedoch nicht ihre absoluten Werte, eine Rolle spielen, werden die Matrixelemente auf ihren Maximalwert normiert. Weiterhin spielt es keine Rolle, ob die Sensitivitäten positiv oder negativ sind und daher wird vor der Normierung elementenweise der Betrag des Gradienten gebildet. Auf diese Weise egeben sich für den Arbeitspunkt

$$
\lambda_{\mathrm{m}, \mathrm{w}}=0,99986, \quad \boldsymbol{\Theta}_{\mathrm{w}}=\left(\begin{array}{lll}
0,87 & 0,9 & 0,9
\end{array}\right)^{\mathrm{T}}
$$

die folgenden drei Matrizen:

$$
\begin{aligned}
& \mathbf{Q}_{\mathrm{O}_{2}}=\operatorname{diag}\left(\begin{array}{lll}
0,45 & 0,91 & 1
\end{array}\right), \\
& \mathbf{Q}_{\mathrm{CO}}=\operatorname{diag}\left(\begin{array}{lll}
0,01 & 0,06 & 1
\end{array}\right), \\
& \mathbf{Q}_{\mathrm{H}_{2}}=\operatorname{diag}\left(\begin{array}{lll}
0,00 & 0,02 & 1
\end{array}\right) .
\end{aligned}
$$

Um beim LQ-Reglerentwurf alle drei Konvertierungsraten zu berücksichtigen, werden die drei Gewichtungsmatrizen $\mathbf{Q}_{\mathrm{O}_{2}}, \mathbf{Q}_{\mathrm{CO}}$ und $\mathbf{Q}_{\mathrm{H}_{2}}$ gewichtet miteinander addiert und die Elemente der resultierenden Matrix auf ihren Maximalwert normiert. Die Gewichtung der Matrizen kann mit den Gewichten $w_{i}$ in Gleichung (6.11) erfolgen, aber auch anhand anderer Überlegungen gewählt werden. Für eine gleiche Gewichtung der ,,mageren“ und der „,fetten“ Schadstoffe, d. h. eine Gewichtung von $\mathbf{Q}_{\mathrm{O}_{2}}$ mit 1 und $\mathbf{Q}_{\mathrm{CO}}$ und $\mathbf{Q}_{\mathrm{H}_{2}}$ mit jeweils 0,5, ergibt sich die folgende Gewichtungsmatrix Q:

$$
\mathbf{Q}=\operatorname{diag}\left(\begin{array}{lll}
0,23 & 0,47 & 1
\end{array}\right)
$$

Stabilität Da der LQ-Regler auf einer Linearisierung des stark nichtlinearen Katalysatormodells basiert, ist es wichtig zu überprüfen, ob er auch für größere Abweichungen vom Arbeitspunkt zu einem stabilen Regelkreis führt. Zur Untersuchung der Stabilität kann wie in Abschnitt 4.2 das Vektorfeld bei einer Diskretisierung des Katalysatormodells in zwei Zellen herangezogen werden. Nach dem oben beschrieben Vorgehen ergibt sich für den Arbeitspunkt

$$
\lambda_{\mathrm{m}, \mathrm{w}}=0,99986, \quad \boldsymbol{\Theta}_{\mathrm{w}}=\left(\begin{array}{ll}
0,87 & 0,9
\end{array}\right)^{\mathrm{T}}
$$

in diesem Fall die Gewichtungsmatrix:

$$
\mathbf{Q}=\operatorname{diag}\left(\begin{array}{ll}
0,3 & 1
\end{array}\right)
$$

Für $R=1$ ist das Vektorfeld des geregelten Katalysatormodells in Bild 6.11 dargestellt. Es ist gut zu erkennen, dass aus jedem Anfangszustand die Zustandstrajektorien in die Ruhelage führen und damit der LQ-Regler zu einem stabilen geschlossenen Regelkreis führt. Der Übersichtlichkeit 
a)

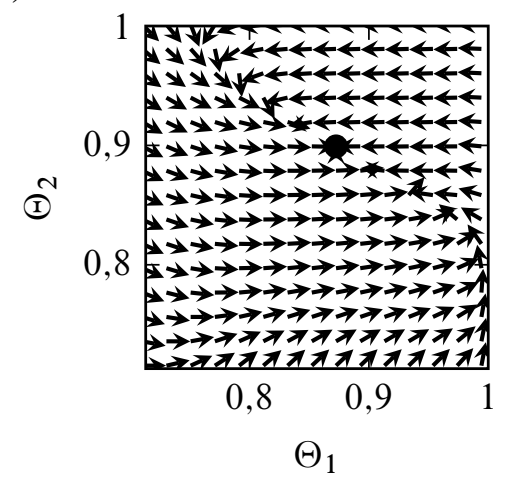

b)

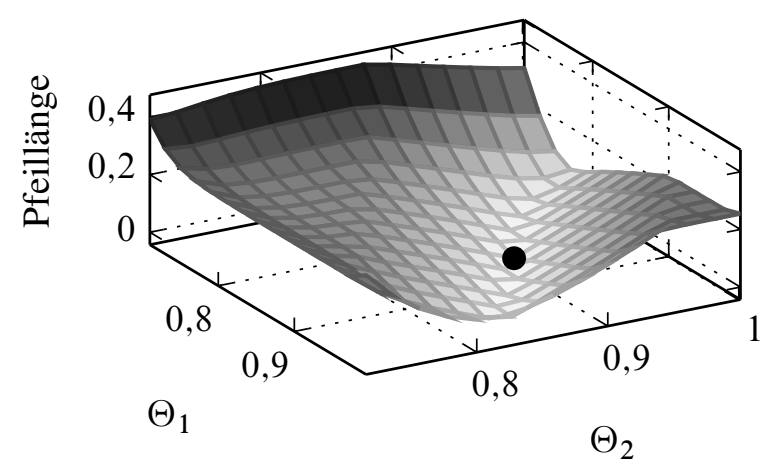

Bild 6.11: Phasenraumanalyse des mit dem LQ-Regler geregelten Katalysators für eine Diskretisierung des Modells in zwei Zellen und die Ruhelage $\boldsymbol{\Theta}=\left(\begin{array}{ll}0,87 & 0,9\end{array}\right)^{\mathrm{T}}$ : a) Vektorfeld; b) Pfeillängen des Vektorfeldes

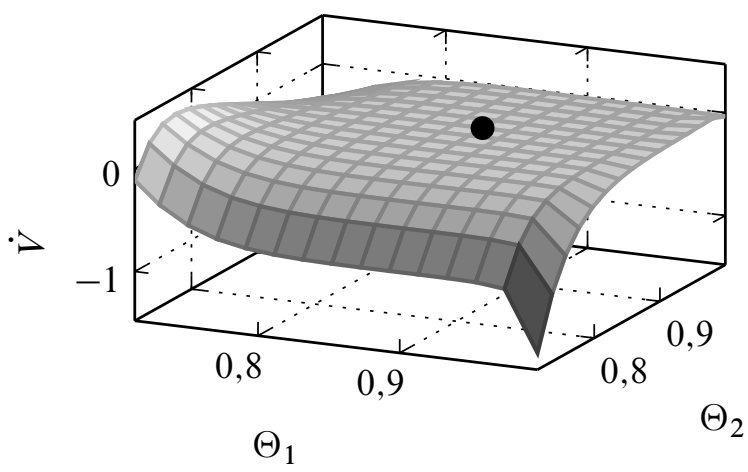

Bild 6.12: Ableitung der Funktion $V$ in Gleichung (6.21)

halber sind die Vektorpfeile in Bild 6.11a auf eine einheitliche Länge skaliert. Die dazugehörigen Pfeillängen sind in Bild 6.11b dargestellt. Im Vergleich zum Vektorfeld des autonomen Systems in Bild 4.2a ist gut zu erkennen, wie der Sauerstofffüllstand der ersten Modellzelle unter- bzw. überschwingt, damit die zweite Modellzelle ihren Sollwert schneller erreicht. Die höhere Dynamik ist an den längeren Pfeillängen in Bild 6.11b im Vergleich zu denen in Bild 4.2b zu erkennen.

Wird mit der Lösungsmatrix $\mathbf{P}$ der algebraischen Riccati-Gleichung, die beim LQ-Reglerentwurf gelöst wird, die quadratische Form

$$
V(\boldsymbol{\Theta})=\boldsymbol{\Theta}^{\mathrm{T}} \mathbf{P} \boldsymbol{\Theta}
$$

gebildet und diese als Ansatz für eine Ljapunov-Funktion eingesetzt, so kann die lokale Stabilität auch analytisch nachgewiesen werden. In Bild 6.12 ist die Ableitung der Funktion $V$ in Gleichung (6.21) dargestellt. Bis auf den kleinen Bereich für niedrige Sauerstofffüllstände der ersten Modellzelle ist die Ableitung von $V$ negativ. Da die Bedingung $\dot{V}<0$ nur hinreichend ist und die Stabilität anhand des Vektorfeldes nachgewiesen werden kann, eignet sich die gewählte Funktion $V$ schlussendlich nicht zum Stabilitätsnachweis im kompletten Arbeitsbereich. Da bei dieser analytischen Stabilitätsanalyse die Dynamik des Lambdaregelkreises außer Acht gelassen worden ist, empfiehlt sich eine vertiefte Stabilitätsanalyse in weiterführenden Arbeiten. 
Simulationen In Bild 6.13 werden drei verschiedene Auslegungen des LQ-Reglers miteinander verglichen. Als Lambdaabweichung wird der synthetische Verlauf aus dem vorherigen Abschnitt verwendet. Neben dem Verlauf der Lambdaabweichung sind in Bild 6.13 noch die Verläufe des Sollwertes des Lambdaregelkreises, der Sauerstofffüllstände des Modells sowie der $\mathrm{O}_{2}$ - und der CO-Konvertierungsrate dargestellt. ${ }^{2}$ Da sich die $\mathrm{H}_{2}$-Konvertierungsrate ähnlich wie die von $\mathrm{CO}$ verhält, ist ihr Verlauf nicht abgebildet. Der erste Regler wird mit der Gewichtungsmatrix $\mathbf{Q}_{\mathrm{O}_{2}}$ in Gleichung (6.15) und dem Faktor $R=2$ entworfen, der zweite mit $\mathbf{Q}_{\mathrm{CO}}$ in Gleichung (6.16) und $R=0,06$ und der dritte mit $\mathbf{Q}$ in Gleichung (6.18) und $R=1$. Der Faktor $R$ wird jeweils so gewählt, dass der Sollwert $\lambda_{\mathrm{w}}$ des Lambdaregelkreises etwa gleich stark genutzt wird. Es fällt auf, dass sich die Faktoren $R$ von Reglerentwurf zu Reglerentwurf stark unterscheiden. Die Regelung des Sauerstofffüllstandes im vorderen Bereich des Katalysators benötigt mehr Stellenergie, da sich die Lambdaabweichung $\lambda_{d}$ stark auf die Sauerstofffüllstände der vorderen Modellzellen auswirkt. Die entsprechenden Regler sind aggressiver und höhere Werte für $R$ werden benötigt, um den Stellaufwand zu reduzieren. Die Regler, die mit den Matrizen $\mathbf{Q}_{\mathrm{O}_{2}}$ und $\mathbf{Q}$ entworfen werden, nutzen die Stellenergie zum Regeln aller Sauerstofffüllstände. Daher lassen sie größere Abweichungen in diesen zu, als der mit der Matrix $\mathbf{Q}_{\mathrm{Co}}$ entworfene Regler. Dennoch können sie den Sauerstofffüllstand im vorderen Bereich des Katalysators besser regeln. Dies ist gut daran zu erkennen, dass die $\mathrm{O}_{2}$-Konvertierungsrate ohne Überschwingen im Vergleich zu dem mit der Matrix $\mathbf{Q}_{\mathrm{CO}}$ entworfenen Regler geregelt wird. Bei der Regelung von $\eta_{\mathrm{CO}}$ verhalten sich alle Regler ähnlich gut, was auf die kleinen Abweichungen des Sauerstofffüllstandes der dritten Modellzelle zurückzuführen ist. Die Verläufe des mit der Matrix $\mathbf{Q}$ entworfenen Reglers verlaufen wie zu erwarten zwischen den Verläufen der anderen beiden Regler und näher an den Verläufen des Reglers mit der Matrix $\mathbf{Q}_{\mathrm{O}_{2}}$. Aufgrund der sehr ähnlichen Stellgrößenausnutzung verhalten sich schlussendlich alle Regler ähnlich. Man kann aber gut erkennen, dass die modellbasierte Wahl von $\mathbf{Q}$ das Regelverhalten in die gewünschte Richtung beeinflusst.

In Bild 6.14 werden drei LQ-Regler miteinander verglichen, die an den drei Motorarbeitspunkten in Anhang A.1 entworfen werden. Am ersten Motorarbeitspunkt wird die Gewichtungsmatrix

$$
\mathbf{Q}_{1}=\operatorname{diag}\left(\begin{array}{lll}
0,53 & 0,51 & 1
\end{array}\right)
$$

verwendet, am zweiten Motorarbeitspunkt die Gewichtungsmatrix $\mathbf{Q}$ in Gleichung (6.18) und am dritten Motorarbeitspunkt die Gewichtungsmatrix:

$$
\mathbf{Q}_{3}=\operatorname{diag}\left(\begin{array}{lll}
0,18 & 0,41 & 1
\end{array}\right) .
$$

Es fällt an der Matrix $\mathbf{Q}_{1}$ in Gleichung (6.22) auf, dass die erste Modellzelle geringfügig stärker als die zweite gewichtet wird. Dies liegt daran, dass am gewählten Arbeitspunkt der Sauerstofffüllstand der ersten Modellzelle geringfügig höher als der der zweiten und der dritten ist. Höhere Sauerstofffüllstände begünstigen die Konvertierung von $\mathrm{O}_{2}$ und daher ergibt sich folgende Gewichtungsmatrix für $\mathrm{O}_{2}$

$$
\mathbf{Q}_{1, \mathrm{O}_{2}}=\operatorname{diag}\left(\begin{array}{lll}
1 & 0,92 & 0,9
\end{array}\right),
$$

\footnotetext{
2 Einige Achsenskalierungen in den folgenden Bildern haben den gleichen Minimalwert und den gleichen Maximalwert. Dies liegt an der Beschränkung der Achsenbeschriftungen auf maximal zwei Nachkommastellen und insgesamt drei signifikante Stellen. Die Änderungen des Lambdawertes und der Füllstände der hinteren Modellzellen spielen sich jedoch in der dritten und die der $\mathrm{O}_{2}$-Konvertierungsrate sogar in der vierten Nachkommastelle ab.
} 

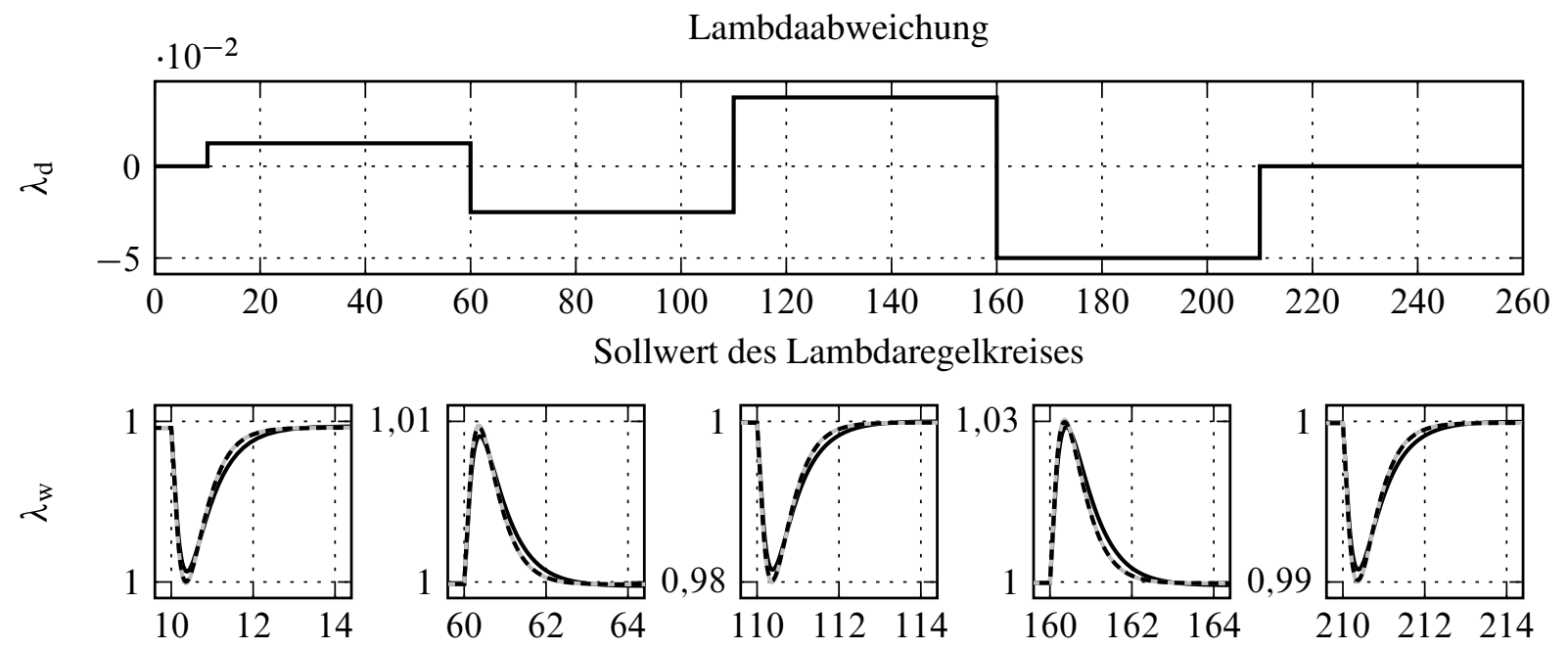

Sauerstofffüllstände der drei Modellzellen
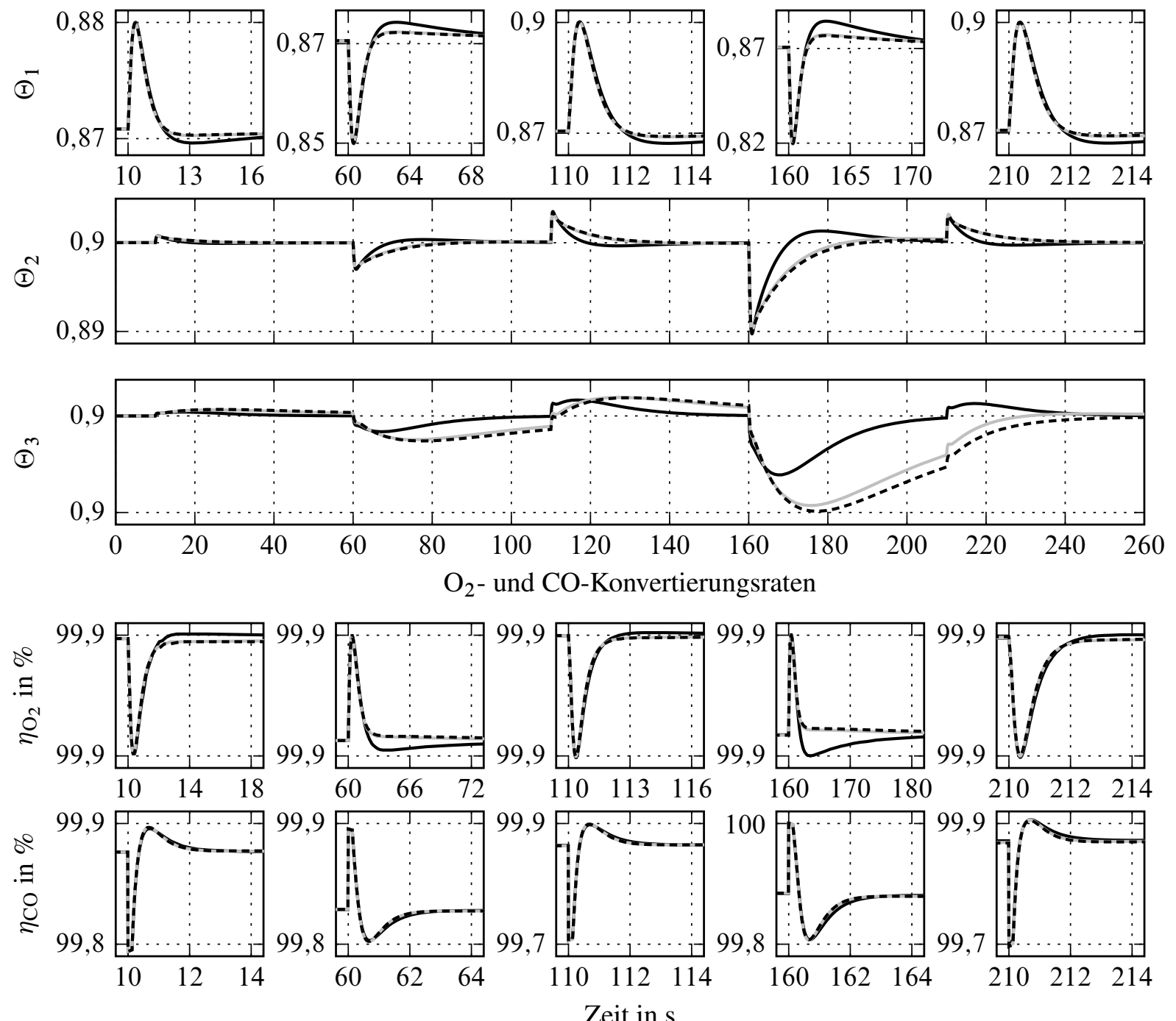

Bild 6.13: Vergleich des LQ-Reglers für drei verschieden Auslegungen am zweiten Motorarbeitspunkt, siehe Anhang A.1: $\mathbf{Q}_{\mathrm{O}_{2}}$ und $R=2(---), \mathbf{Q}_{\mathrm{CO}}$ und $R=0,06(-), \mathbf{Q}$ und $R=1(-)$ 
die für die stärkere Gewichtung der ersten Modellzelle sorgt. Die Regler sind so ausgelegt, dass die Stellgrößenausnutzung stets etwa gleich ist und innerhalb der Stellgrößenbegrenzung von $\pm 10 \%$ bleibt. Wie zu erwarten nehmen die Konvertierungsraten mit steigendem Abgasmassenstrom ab, da die Verweilzeit des Abgases im Katalysator ebenfalls abnimmt. Wie beim Vergleich der mittels exakter Ein-/Ausgangslinearisierung entworfenen Regler im vorherigen Abschnitt werden die Abweichungen der Sauerstofffüllstände am dritten Motorarbeitspunkt am schnellsten und am ersten Motorarbeitspunkt am langsamsten ausgeregelt. Durch die höhere Verweilzeit des Motorabgases im Katalysator am ersten Motorarbeitspunkt haben jedoch die größeren Abweichungen und ihre langsamere Ausregelung keinen signifikanten Einfluss auf die Konvertierungsraten. Die $\mathrm{O}_{2}{ }^{-}$ Konvertierungsrate wird sehr gut geregelt, während diese von CO kleine Ausschläge zu den Zeitpunkten der Sprünge der Lambdaabweichung $\lambda_{d}$ aufweist. Dies ist ein Hinweis darauf, dass selbst wenn die Konvertierungsraten am stärksten vom Sauerstofffüllstand einer Modellzelle abhängen, doch der Sauerstofffüllstand entlang des kompletten Katalysators eine Rolle spielt. Im Gegensatz zum mittels exakter Ein-/Ausgangslinearisierung entworfenen Regler für den ersten Motorarbeitspunkt zeigt der entsprechende LQ-Regler keine Schwingung der Stellgröße.

Zusammenfassung Das analytische Zustandsraummodell erlaubt die Berechnung der absoluten Sensitivität der $\mathrm{O}_{2-}$, der $\mathrm{CO}$ - und der $\mathrm{H}_{2}$-Konvertierungsrate bezüglich der Sauerstofffüllstände der einzelnen Modellzellen. Im Gegensatz zur heuristischen Wahl der Gewichtungsmatrix $\mathbf{Q}$ im Gütemaß des LQ-Reglers in der Literatur wird hier auf Basis der Sensitivitäten eine modellbasierte Wahl dieser Matrix vorgeschlagen. Simulationen zeigen, dass verschiedene Gewichtungen der Gasspezies $\mathrm{O}_{2}, \mathrm{CO}$ und $\mathrm{H}_{2}$ zu verschiedenen LQ-Reglern führen, die das erwünschte Verhalten aufweisen.

Verglichen mit dem mittels exakter Ein-/Ausgangslinearisierung entworfenen Regler ist der LQRegler weniger aggressiv und benötigt weniger Stellgröße, was wichtig für seine Implementierung in der Praxis ist. Des Weiteren entfällt die Problematik mit der Approximation des Totzeitgliedes im Lambdaregelkreis, da dieser nicht berücksichtigt wird. Wird der Katalysator jedoch weiter verkleinert, so wird es nötig sein, den Lambdaregelkreis auch beim LQ-Reglerentwurf zu berücksichtigen. Dafür könnte das Totzeitglied durch eine Padé-Approximation erster Ordnung approximiert werden, die eine höhere Güte als die $\mathrm{PT}_{1}$-Approximation aufweist, siehe Bild 6.3.

\subsubsection{Reglerentwurf mittels exakter Ein-/Ausgangslinearisierung}

Die Analyse der Konvertierungsraten im vorherigen Abschnitt zeigt, dass die $\mathrm{O}_{2-}$, die $\mathrm{CO}$ - und die $\mathrm{H}_{2}$-Konvertierungsrate am stärksten vom Sauerstofffüllstand der dritten Modellzelle abhängen. Die $\mathrm{O}_{2}$-Konvertierungsrate weist jedoch auch eine nicht zu vernachlässigende Abhängigkeit von den Sauerstofffüllständen der beiden vorderen Modellzellen auf. Dieses Verhalten deutet darauf hin, dass der in Abschnitt 6.2.1 entworfene Regler für den Sauerstofffüllstand allein der dritten Modellzelle die $\mathrm{O}_{2}$-Konvertierungsrate nicht sehr gut regeln kann, da die Sauerstofffüllstände der vorderen Modellzellen relativ stark angeregt werden. Umgekehrt ist zu erwarten, dass ein Regler für die $\mathrm{O}_{2}$-Konvertierungsrate auch die von $\mathrm{CO}$ und die von $\mathrm{H}_{2}$ gut regeln wird. Daher ist es 

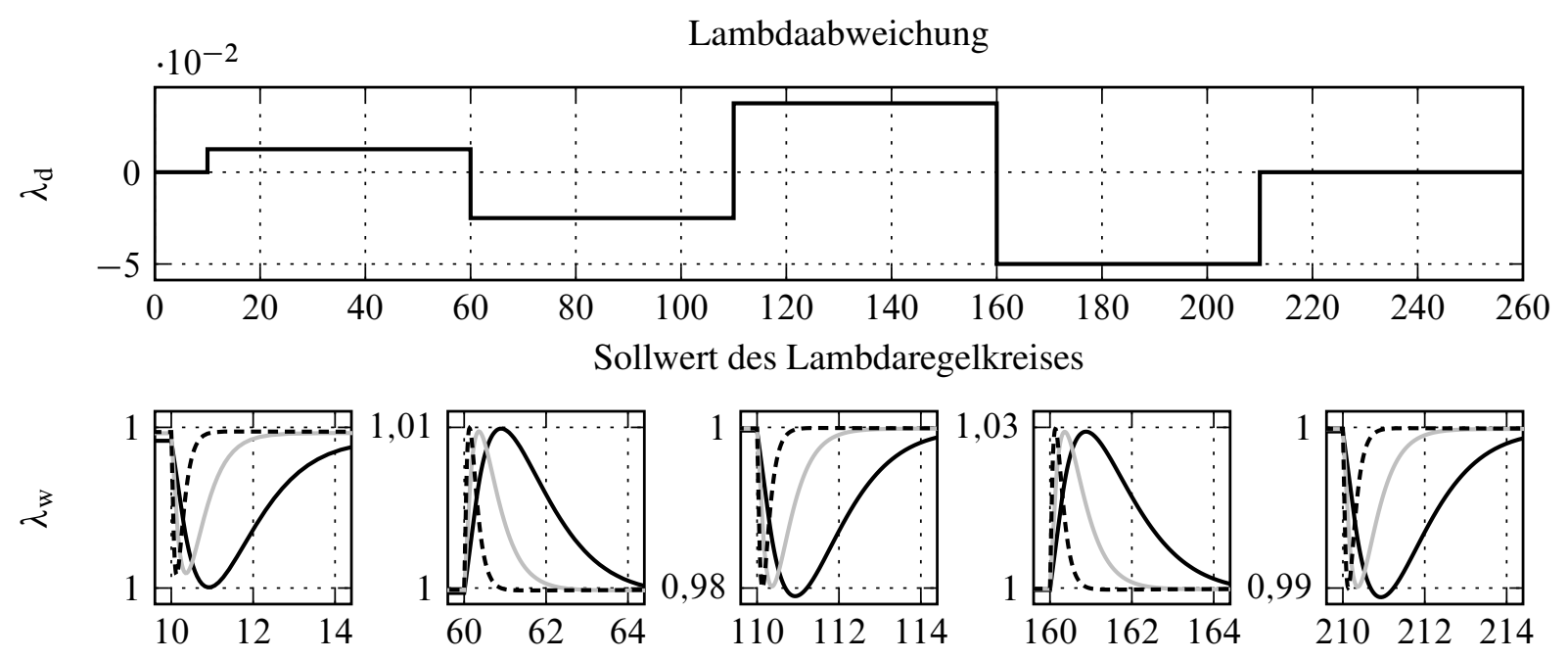

Sauerstofffüllstände der drei Modellzellen
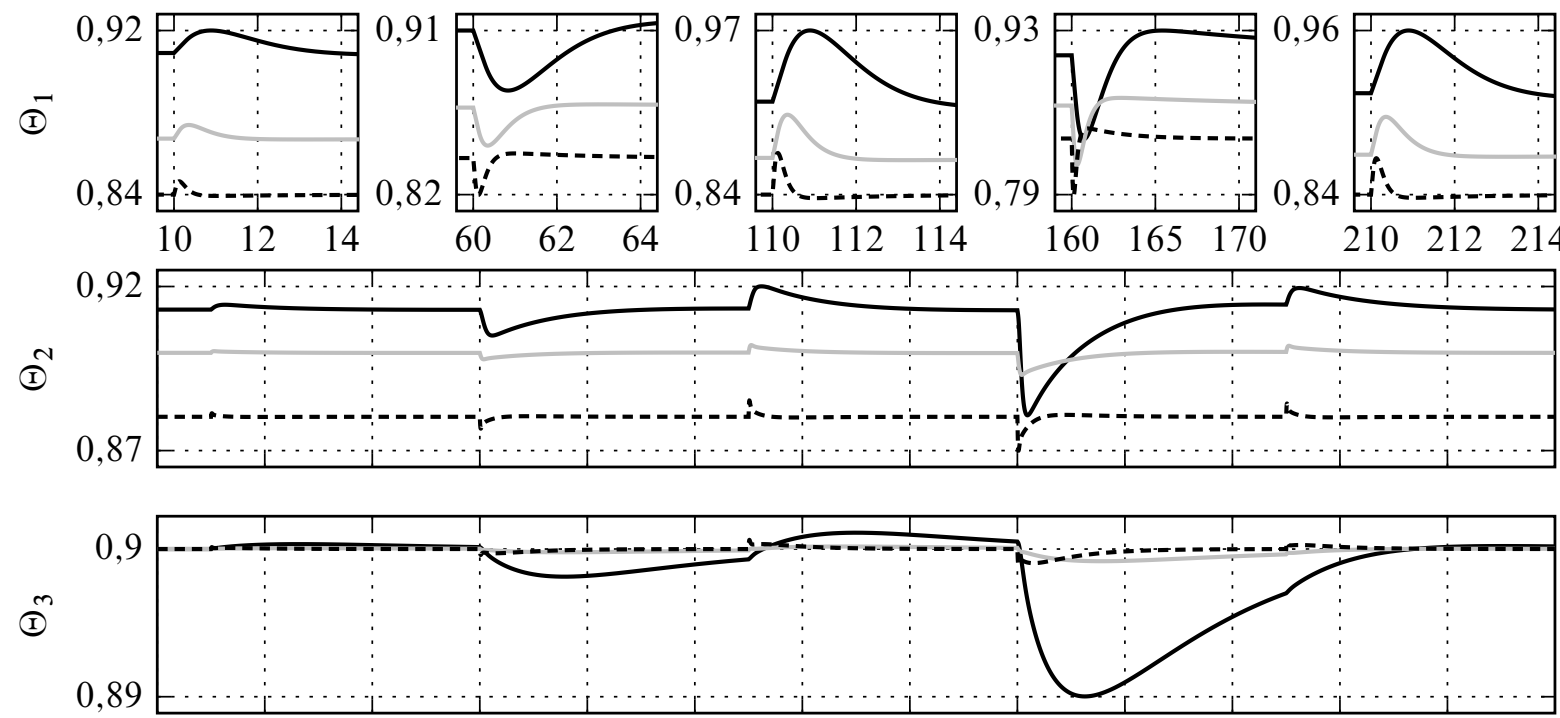

$\mathrm{O}_{2}$ - und CO-Konvertierungsraten
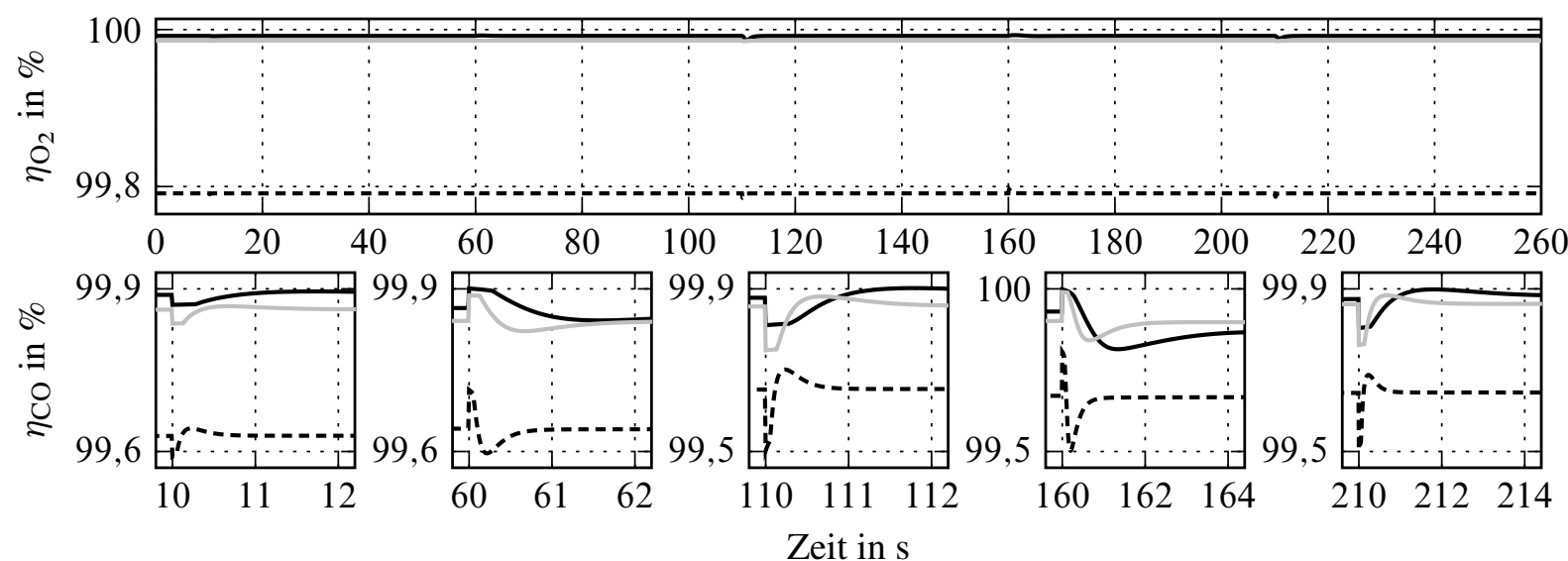

Bild 6.14: Vergleich des LQ-Reglers für eine Auslegung für die drei Motorarbeitspunkte, siehe Anhang A.1: erster Motorarbeitspunkt mit $\mathbf{Q}_{1}$ und $R=5,5(-)$, zweiter Motorarbeitspunkt mit $\mathbf{Q}$ und $R=1(-)$, dritter Motorarbeitspunkt mit $\mathbf{Q}_{\mathbf{3}}$ und $R=0,8(--\cdot-)$ 
naheliegend, einen Regler für $\eta_{\mathrm{O}_{2}}$ mittels exakter Ein-/Ausgangslinearisierung zu entwerfen. Die Ausgangsgleichung (6.2b) wird durch die $\mathrm{O}_{2}$-Konvertierungsrate ersetzt. Nach kurzer Rechnung erhält man den folgenden neuen Ausdruck für $y$ :

$$
y=\eta_{\mathrm{O}_{2}}(\boldsymbol{\Theta})=\underbrace{1-\left(k_{0}-\frac{1}{2} k f_{\mathrm{O}_{2}}\left(\Theta_{3}\right)\right)\left(k_{0}-\frac{1}{2} k f_{\mathrm{O}_{2}}\left(\Theta_{2}\right)\right)\left(k_{0}-\frac{1}{2} k f_{\mathrm{O}_{2}}\left(\Theta_{1}\right)\right)}_{c(\mathbf{x})} .
$$

Die neue Ausgangsgleichung ist deutlich komplexer als die beim bereits durchgeführten Reglerentwurf. Es ist jedoch sofort zu erkennen, dass die Differenzordnung des Katalysatormodells nach wie vor bei Eins liegt. Um die Berechnung der Lie-Ableitungen zu vereinfachen, wird der Lambdaregelkreis einen Schritt weiter vereinfacht, indem er komplett durch ein $\mathrm{PT}_{1}$-Glied approximiert wird. Die Zeitkonstante dieses $\mathrm{PT}_{1}$-Gliedes wird als die Summe der Zeitkonstante $\tau_{\text {imc }}$ und der Totzeit $\tau_{\mathrm{d}}$ gewählt. Damit lautet die Zustandsdifferentialgleichung für den Reglerentwurf:

$$
\frac{\mathrm{d}}{\mathrm{d} t} \underbrace{\left(\begin{array}{c}
\lambda_{\mathrm{d}} \\
\lambda_{\mathrm{imc}, \Sigma} \\
\Theta_{1} \\
\Theta_{2} \\
\Theta_{3}
\end{array}\right)}_{\mathbf{x}}=\underbrace{\left(\begin{array}{c}
0 \\
-\frac{1}{\tau_{\text {imc }}+\tau_{\mathrm{d}}}\left(\lambda_{\text {imc }, \Sigma}+\lambda_{\mathrm{d}}\right) \\
\mathbf{f}^{\mathrm{T}}\left(\Theta_{1}\right) \mathbf{c}^{\mathrm{in}}\left(\lambda_{\text {imc }, \Sigma}+\lambda_{\mathrm{d}}\right) \\
\mathbf{f}^{\mathrm{T}}\left(\Theta_{2}\right) \mathbf{G}_{\mathrm{c}}\left(\Theta_{1}\right) \mathbf{c}^{\mathrm{in}}\left(\lambda_{\text {imc }, \Sigma}+\lambda_{\mathrm{d}}\right) \\
\mathbf{f}^{\mathrm{T}}\left(\Theta_{3}\right) \mathbf{G}_{\mathrm{c}}\left(\Theta_{2}\right) \mathbf{G}_{\mathrm{c}}\left(\Theta_{1}\right) \mathbf{c}^{\mathrm{in}}\left(\lambda_{\text {imc }, \Sigma}+\lambda_{\mathrm{d}}\right)
\end{array}\right)}_{\mathbf{a}(\mathbf{x})}+\underbrace{\left(\begin{array}{c}
0 \\
\frac{1}{\tau_{\mathrm{imc}}+\tau_{\mathrm{d}}} \\
0 \\
0 \\
0
\end{array}\right)}_{\mathbf{b}(\mathbf{x})} \lambda_{\mathrm{w}} .
$$

Hierbei bezeichnet $\lambda_{\text {imc, } \Sigma}$ den Ausgang der $\mathrm{PT}_{1}$-Approximation des Motormodells. Die weiteren Schritte beim Reglerentwurf entsprechen jenen in Abschnitt 6.2.1.

Simulationsstudien zeigen, dass der entworfene Regler die $\mathrm{O}_{2}$-Konvertierungsrate sehr gut regeln kann, allerdings auf Kosten eines noch größeren Stellaufwands im Vergleich zum Regler für den Sauerstofffüllstand der dritten Modellzelle. Die Regelgüte der $\mathrm{O}_{2}$-Konvertierungsrate ist vergleichbar mit der beim LQ-Regler, während die Regelgüten von $\mathrm{CO}$ und $\mathrm{H}_{2}$ je nach Situation etwas schlechter ausfallen. Simulationen für alle drei Motorarbeitspunkte bestätigen die Erwartung, dass bei der großen Zeitkonstante und der großen Totzeit am ersten Motorarbeitspunkt die Stellgröße leicht zu schwingen beginnt. Dieses Verhalten liegt, wie in Abschnitt 6.2.1 beschrieben, in der abnehmenden Approximationsgüte der Totzeit bei hohen Frequenzen. Simulationsergebnisse für den zweiten Motorarbeitspunkt werden in Abschnitt 6.4.1 gezeigt.

\subsubsection{Nichtlineare modellprädiktive Regelung}

Eine modellprädiktive Regelung, Model Predictive Control (MPC), zeichnet sich dadurch aus, dass anhand der zum aktuellen Zeitpunkt gemessenen oder geschätzten Systemzustände eine Stelltrajektorie für die Zukunft mittels Optimierung berechnet wird. Die Stelltrajektorie erstreckt sich über den Prädiktionshorizont $t_{\mathrm{p}}$ und stellt wünschenswerterweise das globale Minimum eines Gütemaßes $J$ dar. Normalerweise wird nur ein kleiner Abschnitt der berechneten Stelltrajektorie, der sogenannte Stellhorizont, verwendet und dann die Optimierung wiederholt [15, 57]. Für das nichtlineare Katalysatormodell wird eine nichtlineare modellprädiktive Regelung (NMPC) implementiert. Sie bietet gegenüber dem bereits entworfenen LQ-Regler mehrere Vorteile: die Möglichkeit 
zur Berücksichtigung des Lambdaregelkreises, der Stellgrößenbeschränkung sowie der nichtlinearen Katalysatordynamik. Die aus der Literatur bekannten NMPC-Implementierungen basieren auf einfacheren, phänomenologischen Katalysatormodellen mit konzentrierten Parametern. Um die Echtzeitfähigkeit der Regler zu gewährleisten, wird eine Neuro-Fuzzy Approximation des Regelgesetzes vorgenommen. Das Ziel des Reglerentwurfs in diesem Abschnitt ist es dahingegen, eine Referenz für die anderen Regelungsverfahren zu erhalten. Daher liegt der Fokus mehr auf der Berücksichtigung des vollständigen nichtlinearen Systemmodells als auf der Echtzeitfähigkeit. Da man zur Lösung des Optimierungsproblems auf numerische Verfahren angewiesen ist, wird eine Diskretisierung der Stelltrajektorie mit der Abtastzeit $\tau$ vorgenommen.

Lösungsverfahren für das Optimierungsproblem In der einfachsten Version einer modellprädiktiven Regelung wird in jedem Abtastschritt ein dynamisches Optimalsteuerungsproblem gelöst, wobei die Vorkenntnisse aus den vorangegangenen Optimierungen nicht explizit genutzt werden. Zur Lösung des dynamischen Optimierungsproblems können indirekte oder direkte Verfahren eingesetzt werden. Bei den indirekten Verfahren resultiert aus den Optimalitäts-, den Rand- und den Transversalitätsbedingungen ein Zwei-Punkt-Randwertproblem. Die dabei notwendige analytische Formulierung des Randwertproblems liefert tiefe Einsichten in die Struktur der optimalen Lösung. Indirekte Verfahren können eine hohe Genauigkeit aufweisen, benötigen meistens jedoch eine Startschätzung der adjungierten Zustände. Das Randwertproblem kann in den wenigsten Fällen analytisch gelöst werden und so ist man auf numerische Lösungsverfahren angewiesen. Direkte Verfahren diskretisieren die Stelltrajektorie und reduzieren das dynamische Optimierungsproblem auf ein statisches Problem. Bei diesen Verfahren brauchen die Optimalitäts-, die Randund die Transversalitätsbedingungen nicht hergeleitet zu werden. Des Weiteren sind vorhandene Stellgrößen- und Zustandsbeschränkungen leichter zu berücksichtigen. Im Vergleich zu den indirekten Verfahren haben direkte Verfahren oft einen größeren Konvergenzbereich [34].

Die Existenz einer Lösung des Optimierungsproblems und der Aufwand zum Auffinden dieser Lösung hängen stark von der Beschaffenheit des Problems ab. Betrachtet man alleine die erste Zelle des Zustandsraummodells (3.39) und berechnet die Hesse-Matrix

$$
\mathbf{H}\left(\mathbf{f}^{\mathrm{T}}\left(\Theta_{1}\right) \mathbf{c}^{\mathrm{in}}\left(\lambda_{\mathrm{m}}\right)\right),
$$

so ist diese indefinit. Das heißt, das Optimierungsproblem ist nicht konvex und es ist nicht garantiert, dass die gefundene Lösung optimal im Sinne des gewählten Gütemaßes ist.

In [118] werden die Optimalitäts-, die Rand- und die Transversalitätsbedingungen für ein in eine, zwei oder drei Zellen diskretisiertes Katalysatormodell aufgestellt. Das dabei entstehende Zwei-Punkt-Randwertproblem wird mit dem Kollokationsverfahren gelöst, das im Löser bvp4c von MATLAB ${ }^{\circledR}$ implementiert ist. Der Lambdaregelkreis wird wie beim Reglerenturf für die $\mathrm{O}_{2}$-Konvertierungsrate im vorherigen Abschnitt durch ein $\mathrm{PT}_{1}$-Glied approximiert. Als Gütemaß wird, bis auf kleine Änderungen, das quadratische Gütemaß (6.12) wie beim LQ-Reglerentwurf eingesetzt. Die obere Integrationsgrenze wird durch den Prädiktionshorizont $t_{\mathrm{p}}$ ersetzt, der $\mathrm{Zu}-$ stand des Lambdaregelkreises wird nicht gewichtet und zur Gewichtung der Sauerstofffüllstände dient wieder die Matrix $\mathbf{Q}$ in Gleichung (6.18). Das Optimalsteuerungsproblem kann mit dem 


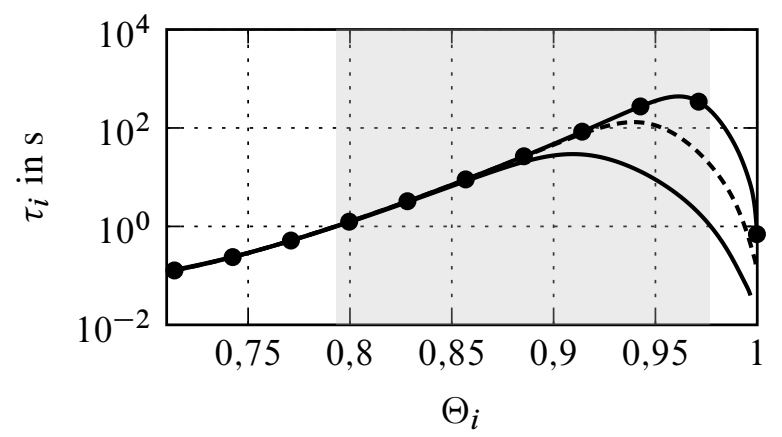

Bild 6.15: Zeitkonstanten des Katalysatormodells am zweiten Motorarbeitspunkt, siehe Anhang A.1, und Bereich (grau), in dem die Zeitkonstanten größer als $1 \mathrm{~s}$ sind: 1. Modellzelle $(-)$, 2. Modellzelle (----), 3. Modellzelle (-๑)

indirekten Verfahren zwar gelöst werden, die Wahl der Startschätzung der adjungierten Zustände erschwert jedoch die unbeaufsichtigte Simulation der modellprädiktiven Regelung. Daher und auch um die Approximation des Lambdaregelkreises zu umgehen, wird ein indirektes Verfahren zum Lösen des Optimierungsproblems eingesetzt, die in IPOPT implementierte Innere-PunkteMethode [109]. Die Eingabe des Optimierungsproblems und die Ansteuerung des Lösers erfolgen über die frei verfügbare MATLAB ${ }^{\circledR}$ Toolbox YALMIP [56]. Dabei wird eine Volldiskretisierung durchgeführt und das Integral im Gütemaß durch eine Summe über die $N$ Abtastschritte des Prädiktionshorizonts ersetzt. Beim direkten Lösungsverfahren ist es denkbar und sinnvoll, andere als das quadratische Gütemaß einzusetzen. Zum Beispiel könnten die $\mathrm{O}_{2^{-}}$, die $\mathrm{CO}$ - und die $\mathrm{H}_{2^{-}}$ Konvertierungsrate direkt in das Gütemaß eingehen. Da der Entwurf der NMPC nicht der Hauptgegenstand dieser Arbeit ist, beschränkt sich der Entwurf hier auf das quadratische Gütemaß. Die Freiheitsgrade des Entwurfs sind die Länge des Prädiktionshorizonts, das Integrationsverfahren und die Integrationsschrittweite für die Simulation des Modells sowie der Faktor $R$ zu Gewichtung des Stellaufwands. Für den zweiten Motorarbeitspunkt stellt sich die Wahl von $R=1$ als geeignet heraus.

In der Literatur ist keine eindeutige Regel zur Wahl des Prädiktionshorizonts zu finden. Der Prädiktionshorizont sollte zum einen lang genug sein, damit die Auswirkungen der Stellgrößeneingriffe erfasst werden können, und zum anderen möglichst kurz sein, damit der Rechenaufwand gering gehalten wird. In [57] wird eine Horizontlänge des Zwei- bis Dreifachen der dominanten Systemzeitkonstante vorgeschlagen. Wird der Prädiktionshorizont etwas reduziert und gleich der maximalen Zeitkonstante des Katalysatormodells gewählt und gleichzeitig eine Integrationsschrittweite gewählt, die kleiner als die minimale Zeitkonstante ist, so entsteht ein sehr großes Optimierungsproblem, das nicht praktikabel zu lösen ist. Aus diesem Grund wird anhand von Simulationsstudien die Länge des Prädiktionshorizonts auf einen Wert im Bereich von 5 bis 8 Sekunden und die Integrationsschrittweite auf $100 \mathrm{~ms}$ festgelegt. Orientiert man sich an der Faustregel, dass die Simulationsschrittweite um das Fünf- bis Zehnfache kleiner als die kleinste Systemzeitkonstante sein soll, so wird die Simulation des Modells nur in einem eingeschränkten Sauerstofffüllstandsbereich korrekt durchgeführt. In Bild 6.15 sind die Zeitkonstanten des Katalysatormodells am zweiten Motorarbeitspunkt und der Bereich dargestellt, in dem die Zeitkonstanten größer als 
eine Sekunde sind. Die hier getroffene Wahl der Integrationsschrittweite führt somit zu größeren Simulationsfehlern, wenn sich der Sauerstofffüllstand einer Modellzelle am Rand des Arbeitsbereichs befindet. Aufgrund der asymptotischen Stabilität des Katalysators und der Verwendung der Zustände des Open-Loop-Beobachters als Anfangszustände in jedem Optimierungsschritt, sind die Auswirkungen der Integrationsfehler jedoch klein. Die größere Integrationsschrittweite hat auch den Vorteil, dass die Totzeit im Lambdaregelkreis nur durch einen bis drei Abtastschritte dargestellt werden kann.

Simulationsergebnisse für den zweiten Motorarbeitspunkt werden in Abschnitt 6.4.1 gezeigt.

\subsection{Vergleich der Regelungskonzepte}

In diesem Abschnitt werden die in den Abschnitten 6.2 und 6.3 vorgestellten Regler zuerst anhand von Simulationen miteinander verglichen. Der nichtlineare Regler für den Sauerstofffüllstand der dritten Modellzelle und der LQ-Regler sind zusammen mit der Lambda-Eins-Regelstrategie auch am Laborprüfstand implementiert worden. Im zweiten Teil des Abschnitts werden die Testszenarien erläutert und die Regler anhand von Messdaten miteinander verglichen.

\subsubsection{Vergleich mit Simulationsdaten}

Für den Vergleich in der Simulationsumgebung wird der zweite Motorarbeitspunkt und die synthetische Lambdaabweichung in Abschnitt 6.2.1 gewählt. Zwar stehen reale Messdaten vom Motorprüfstand zur Verfügung, die auch zur Verifikation der Regler genutzt werden können, siehe Abschnitt 2.2.1, jedoch sprechen zwei Gründe dagegen. Zum einen ist die dynamische Adaption der Emissionsregler an den Motorarbeitspunkt nicht im Detail untersucht worden und zum anderen stellt das letzte Drittel des FTP 75-Fahrzyklus keine anspruchsvolle Anregung dar, sodass der Vergleich erschwert wird.

In Bild 6.16 sind die Verläufe der synthetischen Lambdaabweichung $\lambda_{\mathrm{d}}$, des Soll-Lambdawertes $\lambda_{w}$ des Lambdaregelkreises, der Sauerstofffüllstände des Modells sowie der $\mathrm{O}_{2}$ - und der CO-Konvertierungsrate dargestellt. ${ }^{3}$ Der Sollwert des Lambdaregelkreises bleibt bei der Lambda-EinsRegelstrategie unabhängig von der Lambdaabweichung konstant. Die Nichtberücksichtigung der Katalysatordynamik führt, wie in Abschnitt 6.2.1 beschrieben, zu großen Abweichungen der Sauerstofffüllstände und damit der Konvertierungsraten von ihren Sollwerten im Vergleich zu den anderen Reglern.

Die Regelung des Sauerstofffüllstandes der dritten Modellzelle mittels exakter Ein-/Ausgangslinearisierung zeichnet sich durch eine sehr hohe Regelgüte aus, die allerdings durch einen hohen Stellaufwand und damit eine stärkere Anregung der Sauerstofffüllstände der vorderen Modellzellen erkauft wird. Diese starke Anregung hat zur Folge, dass die $\mathrm{O}_{2}$-Konvertierungsrate deutlich

\footnotetext{
${ }^{3}$ Siehe den Hinweis in Fußnote 2 auf Seite 105.
} 

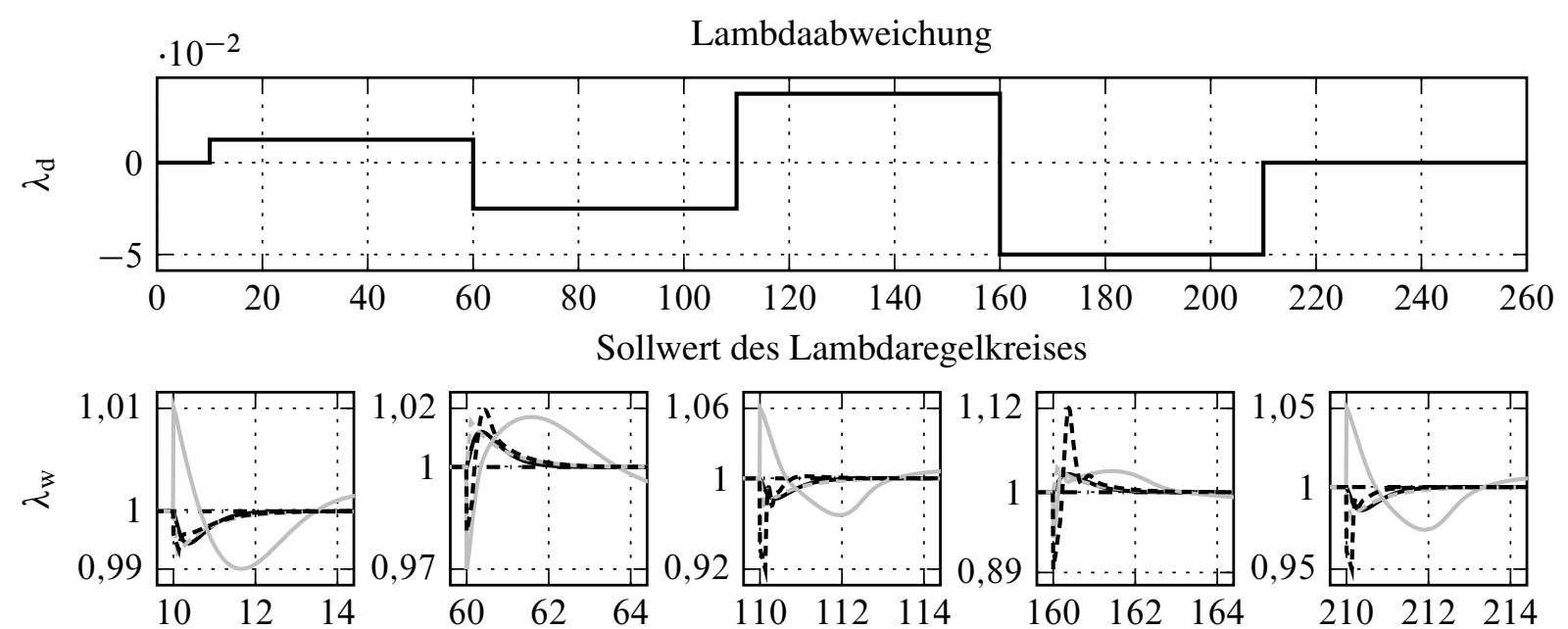

Sauerstofffüllstände der drei Modellzellen
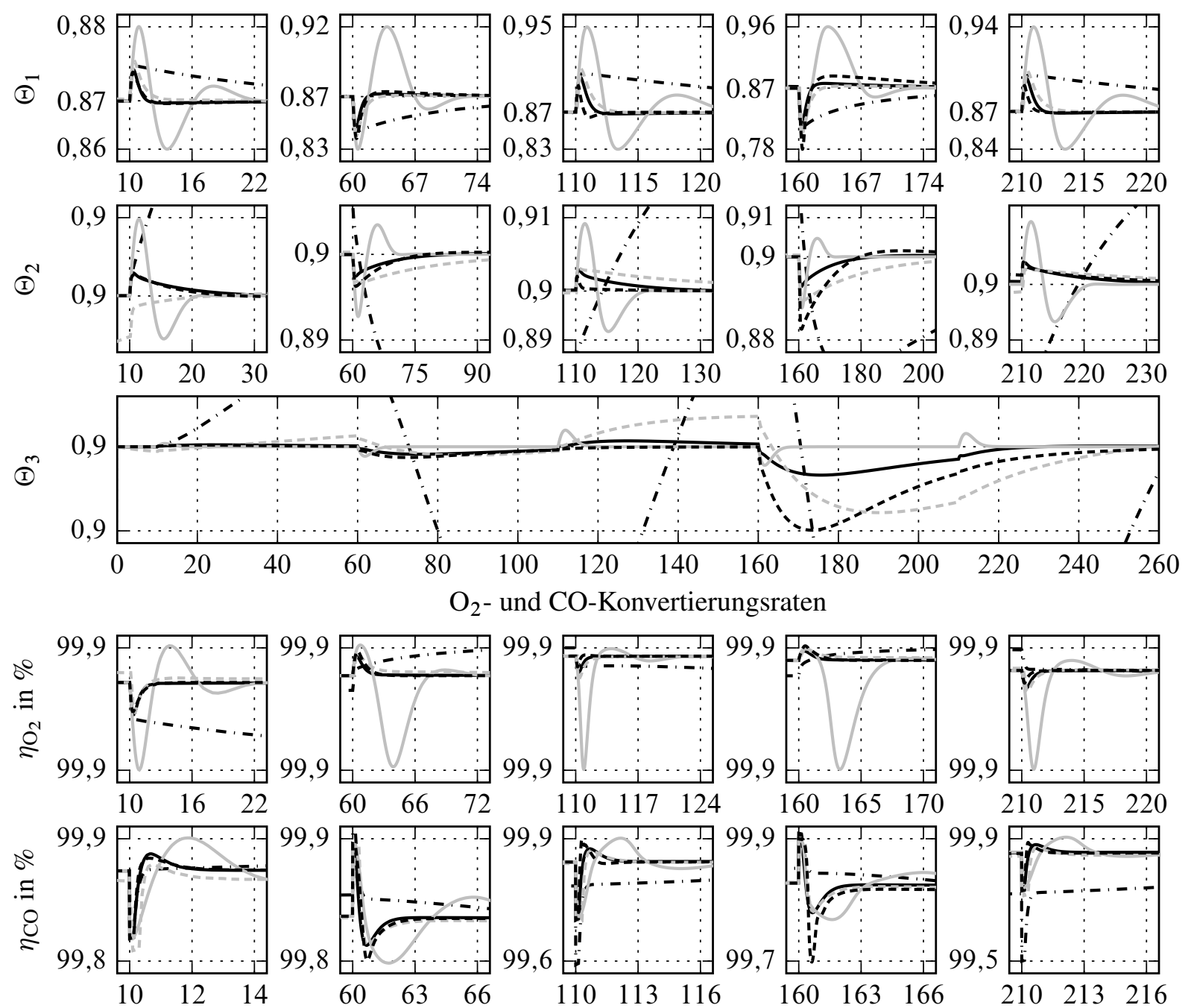

Zeit in $\mathrm{s}$

Bild 6.16: Vergleich der vier entworfenen Regler und der Lambda-Eins-Regelstrategie anhand der synthetischen Lambdaabweichung am zweiten Motorarbeitspunkt, siehe Anhang A.1: Lambda-Eins-Regelstrategie (---), LQ-Regler (-), exakte Ein-/Ausgangslineariesierung für $\Theta_{3}(-)$ und für $\eta_{\mathrm{O}_{2}}(----)$ sowie nichtlineare modellprädiktive Regelung (---) 
stärker als bei den anderen Reglern einbricht, während die CO-Konvertierungsrate wie zu erwarten gut geregelt wird.

Obwohl der LQ-Regler die dritte Modellzelle am stärksten gewichtet, werden im Vergleich zum nichtlinearen Regler größere Abweichungen zugelassen, welche auch deutlich langsamer ausgeregelt werden. Dies ist insbesondere beim größten Sprung der Lambdaabweichung bei $160 \mathrm{~s}$ zu sehen. Die Regelgüte des LQ-Reglers hängt stark davon ab, wie stark die Lambdaabweichung den Systemzustand vom gewählten Arbeitspunkt auslenkt. Dieses Verhalten hängt damit zusammen, dass der LQ-Regler anhand einer Linearisierung des stark nichtlinearen Katalysatormodells entworfen wird. Im Gegensatz dazu zeigt der nichtlineare Regler eine ähnliche Regelgüte bei jedem Sprung der Lambdaabweichung. Da jedoch im Gütemaß des LQ-Reglers alle Modellzellen berücksichtigt werden, unterliegen die Sauerstofffüllstände der vorderen Zellen kleineren Abweichungen. Dies führt dazu, dass sowohl die $\mathrm{O}_{2}$-Konvertierungsrate als auch die von $\mathrm{CO}$ am besten geregelt werden. Offensichtlich wirkt sich die langsamere Ausregelung der Abweichung des Sauerstofffüllstandes der dritten Modellzelle nicht negativ auf die Schadstoffkonvertierung aus, was auch an den kleinen absoluten Werten der Abweichung liegt.

Die Regelung der $\mathrm{O}_{2}$-Konvertierungsrate mittels exakter Ein-/Ausgangslinearisierung funktioniert sehr gut und ähnlich gut wie beim LQ-Regler, allerdings auf Kosten eines sehr aggressiven Stellgrößenverlaufs. Die sehr aggressive Regelung führt schließlich zu kleinen Einbrüchen in der COKonvertierungsrate. Die nichtlineare modellprädiktive Regelung verhält sich ähnlich wie der LQRegler, was am gleichen Gütemaß liegt, lässt jedoch etwas größere Abweichungen der Sauerstofffüllstände von den Sollwerten zu.

Diskussion Die Simulationsergebnisse zeigen, dass die Berücksichtigung der Katalysatordynamik beim Entwurf des Emissionsreglers sinnvoll ist und zu einer Verbesserung der Schadstoffkonvertierung gegenüber Lambda-Eins-Regelstrategien führt. Beim Katalysator handelt es sich um ein System mit einem Eingang und einem Ausgang und daher kann nur eine Regelgröße gewählt werden. Dies ist bei der exakten Ein-/Ausgangslinearisierung insoweit problematisch, da beim Reglerentwurf vorzugsweise alle drei Konvertierungsraten berücksichtigt werden sollten. Durch die Systeminversion wird die komplette nichtlineare Systemdynamik kompensiert, was beim Katalysator einen großen Stellaufwand erfordert. Dem nichtlinearen System wird eine lineare Dynamik aufgezwungen und dadurch werden offensichtlich auch nützliche Nichtlinearitäten kompensiert. Die Wahl der $\mathrm{O}_{2}$-Konvertierungsrate als Regelgröße ist ein interessanter Ansatz, allerdings steigt dabei der Stellaufwand weiter. Ein weiteres Problem beim Reglerentwurf mittels Systeminversion stellt die minimalphasige Approximation des Totzeitgliedes im Lambdaregelkreis dar. Die Approximationsgüte des hier eingesetzten $\mathrm{PT}_{1}$-Gliedes nimmt mit steigender Totzeit ab und führt z. B. bei niedriger Motordrehzahl zu kleinen Schwingungen der Stellgröße. Des Weiteren begrenzt die Problematik der nicht konsistenten Differenzordnung die Zellenanzahl des Katalysatormodells auf maximal drei.

Bezogen auf die in der Praxis relevanten Konvertierungsraten erzielt der LQ-Regler die beste Regelgüte und stellt gleichzeitig den am einfachsten zu implementierenden Regler dar. Im Bereich 
Tabelle 6.1: Qualitativer Vergleich der entworfenen Regler

\begin{tabular}{|c|c|c|c|}
\hline & $\begin{array}{l}\text { exakte Ein-/ } \\
\text { Ausgangslinearisierung }\end{array}$ & LQ-Regler & $\begin{array}{l}\text { Nichtlineare modellprädiktive } \\
\text { Regelung }\end{array}$ \\
\hline Regelziel & $\Theta_{3}$ oder $\eta_{\mathrm{O}_{2}}$ & \multicolumn{2}{|c|}{$\begin{array}{l}\text { Minimierung von } J \text {, Berücksichtigung } \\
\text { von } \eta_{\mathrm{O}_{2}}, \eta_{\mathrm{CO}} \text { und } \eta_{\mathrm{H}_{2}}\end{array}$} \\
\hline Regelgüte & $\begin{array}{l}\text { sehr gut }\left(\Theta_{3}\right) \\
\text { sehr gut }\left(\eta_{\mathrm{O}_{2}}\right)\end{array}$ & gut & \\
\hline Lambdaregelkreis & Approximation & $\begin{array}{l}\text { keine } \\
\text { Berücksichtigung }\end{array}$ & $\begin{array}{l}\text { vollständige } \\
\text { Berücksichtigung }\end{array}$ \\
\hline Komplexität & mittel & niedrig & hoch \\
\hline Rechenaufwand & niedrig & niedrig & hoch \\
\hline Zellenanzahl & bis zu 3 & $\begin{array}{l}\text { auch mehr als } 3 \\
\text { möglich }\end{array}$ & bis $\mathrm{zu} 3$ \\
\hline
\end{tabular}

des Arbeitspunktes sind die Zeitkonstanten des Katalysators groß und der LQ-Reglerentwurf zielt auf die lokale Verbesserung der Systemdynamik in diesem Bereich. Dennoch resultiert dieser lokale Reglerentwurf in Kombination mit der asymptotischen Stabilität des Katalysators im gesamten Arbeitsbereich in einem stabilen geschlossenen Regelkreis und einem geringen StellgröBenbedarf. Es ist in diesem Kontext auch eine Diskretisierung des Katalysatormodells in mehr als drei Zellen denkbar. Jedoch wird es mit steigender Zellenanzahl aus numerischer Sicht immer schwieriger, den stationären Arbeitspunkt zu berechnen.

Die Implementierung der nichtlinearen modellprädiktiven Regelung auf Basis des physikochemischen Modells und die Berücksichtigung von bis zu drei Modellzellen wird erst durch die neue, kompakte Darstellung des Zustandsraummodells möglich. Zwar liefert der gegenwärtige Entwurf eine geringfügig schlechtere Regelgüte als der LQ-Regler, bietet aber prinzipiell die Möglichkeit, auch andere Gütemaße zu verwenden.

In Tabelle 6.1 werden die wichtigsten Eigenschaften der in dieser Arbeit entworfenen Regler qualitativ miteinander verglichen. Die Regelgüte bezieht sich dabei auf die gewählte Regelgröße. Die Minimierung des Gütemaßes beim LQ-Regler und der NMPC impliziert die Regelung aller drei Sauerstofffüllstände. Aufgrund der Problematik mit der Totzeit und des hohen Stellaufwands ist bei der praktischen Implementierung der LQ-Regler den auf Systeminversion basierenden Reglern vorzuziehen.

\subsubsection{Vergleich mit Messdaten}

Bei den ersten Messungen am Laborprüfstand sind eine Temperatur von $850 \mathrm{~K}$ und eine Raumgeschwindigkeit von $50000 \mathrm{~h}^{-1}$ gewählt worden, die auch in der Praxis realistisch sind, siehe Anhang A.1. Da der verwendete Katalysator bei niedrigen Raumgeschwindigkeiten sehr gut $\mathrm{NO}_{\mathrm{x}}$ und HC umsetzten kann, siehe Abschnitt 5.3, ist im Laufe des Projekts mehr und mehr zu einer 
Temperatur von $773 \mathrm{~K}$ und einer Raumgeschwindigkeit von $100000 \mathrm{~h}^{-1}$ übergegangen worden. Damit wird die Pufferwirkung des Sauerstoffspeichers reduziert und die Unterschiede zwischen den Reglern können besser untersucht werden. In diesem Abschnitt werden drei Testszenarien präsentiert und die Lambda-Eins-Regelstrategie, der LQ-Regler und der nichtlineare Regler für den Sauerstofffüllstand der dritten Modellzelle miteinander verglichen. Im ersten Testszenario wird eine synthetische Lambdaabweichung eingesetzt, das zweite Testszenario stellt die Situation nach dem Wiedereinschalten der Kraftstoffeinspritzung nach einer Phase mit Schubabschaltung (fuel cut-off) nach und im dritten Testszenario wird der Einfluss der Stellgrößenbeschränkung untersucht. In Anhang A.2 sind Informationen über den Laborprüfstand und seine Ausstattung zu finden.

Synthetische Lambdaabweichung In Bild 6.17 sind die Verläufe der synthetischen Lambdaabweichung, der Stellgröße des Lambdaregelreises, der Spannung $U_{\text {su }}$ der Sprung-Lambdasonde hinter dem Katalysator sowie der CO-Konzentration im Abgas hinter dem Katalysator dargestellt. Aufgrund der messtechnisch nicht erfassbaren Unterschiede in der NO-Konzentration zwischen den Reglern wird auf die entsprechende Darstellung verzichtet. Die Lambdaabweichung ist so gewählt, dass die Sprunghöhe im Vergleich zu der synthetischen Lambdaabweichung in den Simulationen mit einem Prozent pro Sprung langsamer wächst. Die maximale Sprunghöhe von $12 \%$ wird bei $710 \mathrm{~s}$ angenommen. Dem Bild ist zu entnehmen, dass die Lambda-Eins-Regelstrategie zu den höchsten CO-Emissionen und größten Abweichungen der Spannung $U_{\text {su }}$ vom Sollwert von $650 \mathrm{mV}$ führt. Die Maximalwerte der Abweichungen steigen mit der Sprunghöhe stark an.

Der LQ-Regler und der mittels exakter Ein-/Ausgangslinearisierung entworfene Regler zeigen dagegen eine deutlich höhere Regelgüte und lassen die Spannung $U_{\text {su }}$ um maximal $\pm 15 \mathrm{mV}$ vom Sollwert abweichen. ${ }^{4}$ Dabei ist zu erkennen, dass der nichtlineare Regler etwas schneller reagiert, da er die im Lambdaregelkreis geschätzte Lambdaabweichung direkt berücksichtigt, während der LQ-Regler erst auf Änderungen der geschätzten Sauerstofffüllstände reagiert. Der nichtlineare Regler zeichnet sich wie erwartet durch einen im Vergleich zum LQ-Regler aggressiveren Stellgrößenverlauf aus. Es ist einerseits bemerkenswert, dass zwei so unterschiedliche Regelstrategien zu einem so ähnlichen Verlauf der Spannung $U_{\text {su }}$ führen, und andererseits hilfreich zu verstehen, dass die Spannung $U_{\text {su }}$ als einziges Maß für die Regelgüte nicht geeignet ist. In die Berechnung des Lambdawertes fließen alle Gaskonzentrationen ein und zusammen mit den komplexen Reaktionen in der Lambdasonde selbst kann nicht darauf geschlossen werden, wie sich einzelne Gaskonzentrationen verhalten. Am Verlauf der CO-Konzentration ist gut zu sehen, dass der LQ-Regler deutlich höhere CO-Konzentrationsspitzen als der nichtlineare Regler zulässt. Interessanterweise wirken sich diese höheren Konzentrationen nicht unmittelbar auf die Spannung $U_{\text {su }}$ aus. Größere Spannungsabweichungen korrelieren dennoch mit höheren CO-Emissionen. Die Regelung des Sauerstofffüllstandes im hinteren Bereich des Katalysators entspricht nach der Analyse der Konvertierungsraten in Abschnitt 6.3.1 einer Regelung der $\mathrm{CO}$ - und der $\mathrm{H}_{2}$-Konvertierungsrate. Genau dieses Verhalten ist in Bild $6.17 \mathrm{zu}$ beobachten. Die CO-Konzentration beim Einsatz des

\footnotetext{
${ }^{4}$ Der Übersichtlichkeit halber ist in der vergrößerten Darstellung der Spannungsverlauf der Lambda-EinsRegelstrategie weggelassen worden.
} 

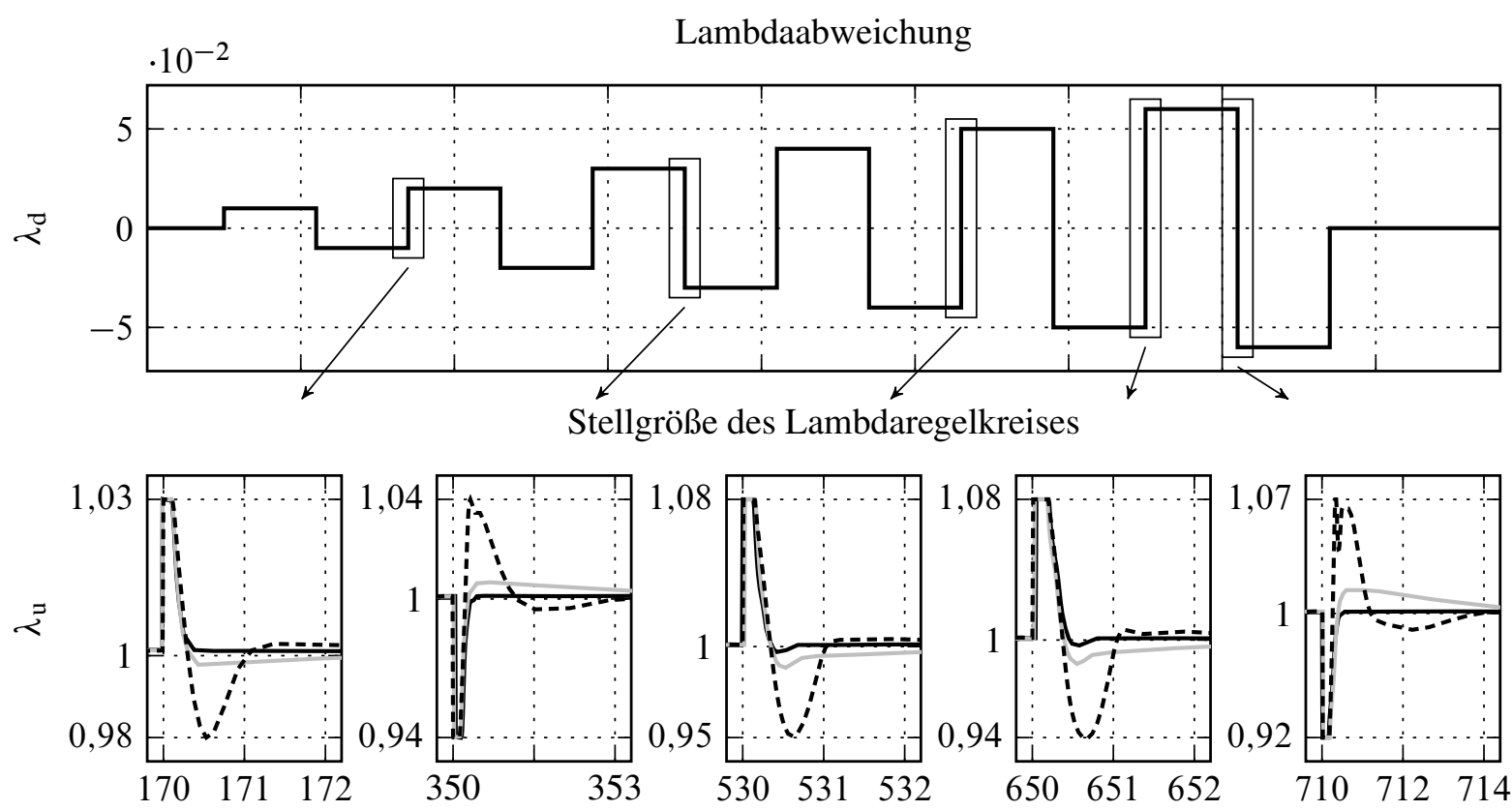

Spannung der Sprung-Lambdasonde
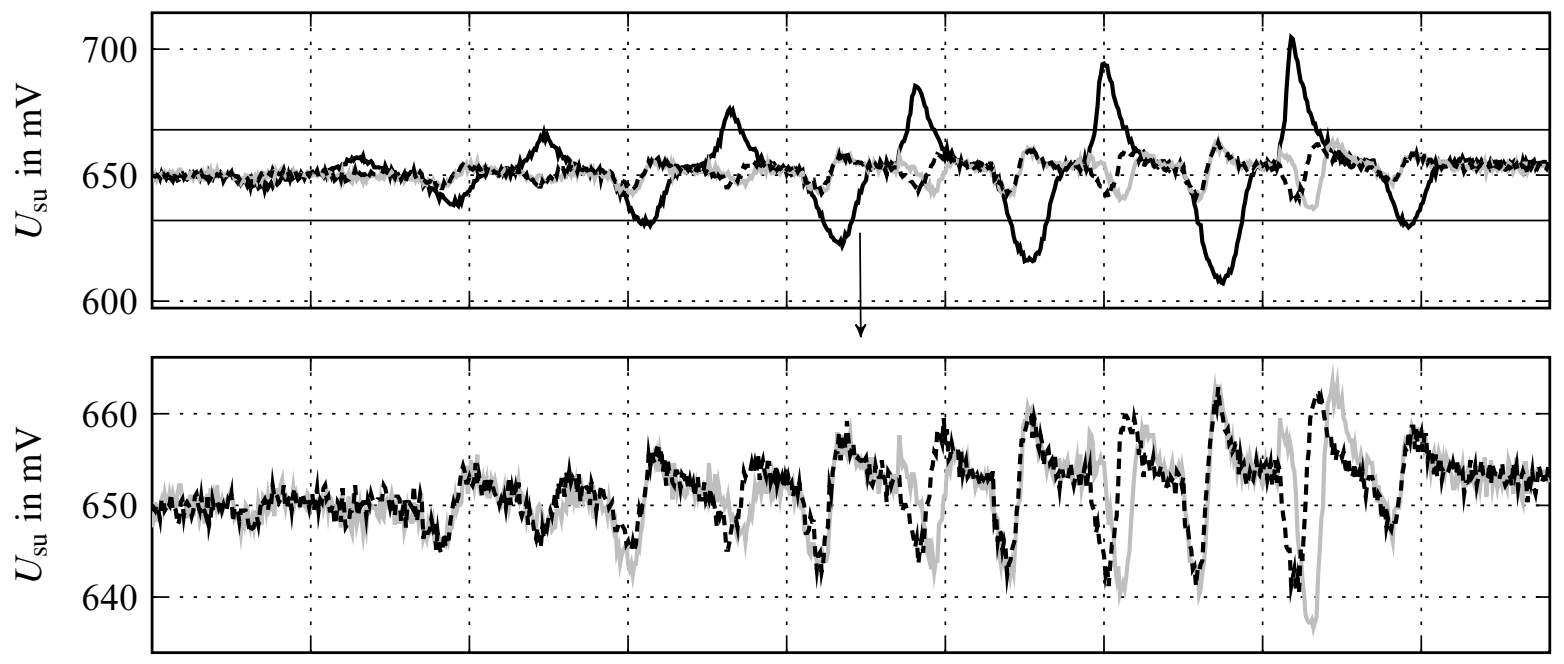

CO-Konzentrationen

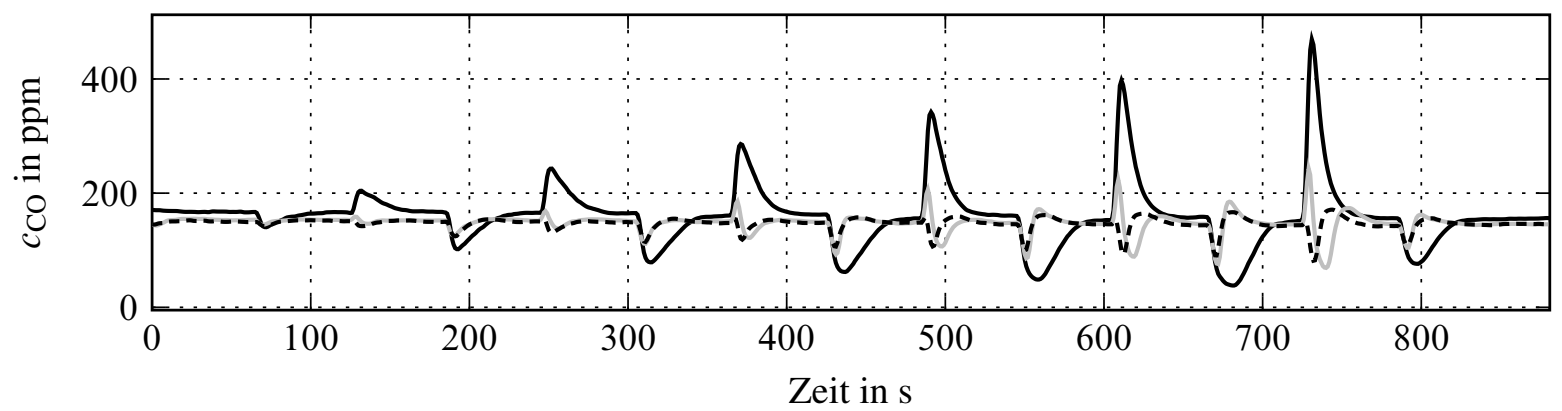

Bild 6.17: Vergleich der Lambda-Eins-Regelstrategie ( - ), des LQ-Reglers $(-)$ und der exakten Ein-/Ausgangslinearisierung für $\Theta_{3}$ (----) anhand einer synthetischen Lambdaabweichung. Die Temperatur beträgt $850 \mathrm{~K}$ und die Raumgeschwindigkeit $50000 \mathrm{~h}^{-1}$. 
nichtlinearen Reglers steigt nur wenig über den Sollwert und wird am schnellsten geregelt. Diese Korrelation zwischen den theoretischen Untersuchungen, dem Regelziel des nichtlinearen Reglers und den Messergebnissen sprechen für die Güte des Modells und der gemachten Analysen. Das langsame Nachschwingen der Spannung $U_{\text {su }}$ bei den Emissionsreglern kann daran liegen, dass die komplexe Katalysatordynamik nur durch wenige Reaktionsgleichungen angenähert wird und die Modelldiskretisierung mit nur drei Zellen erfolgt. Daher können nicht alle Effekte berücksichtigt werden, die aber offensichtlich keine großen negativen Auswirkungen aufweisen.

Fuel cut-off Wenn z. B. im Motorschubbetrieb oder bei einem Gangwechsel bei einem Fahrzeug mit Schaltgetriebe die Kraftstoffeinspritzung vom Motorsteuergerät unterbrochen wird, fördert der Motor Luft durch den Katalysator und befüllt damit seinen Sauerstoffspeicher. Beim Wiedereinschalten der Kraftstoffeinspritzung muss der Sauerstofffüllstand so schnell wie möglich wieder auf seinen mittleren Wert gebracht werden, damit der Katalysator auf magere und fette Lambdaabweichungen vorbereitet ist. Beim üblichen Ansatz in der Literatur wird während des Motorschubbetriebs die Emissionsregelung ausgeschaltet und im ersten Moment nach dem Wiedereinschalten der Kraftstoffzufuhr wird eine separate Steuerstrategie verwendet. Sobald der Sauerstofffüllstand in die Nähe des gewählten Arbeitspunktes kommt, wird die Emissionsregelung wieder eingeschaltet, siehe die Arbeiten von Kiwic u. a. [50, 51], Möller [64] und Auckenthaler [4]. Der mittels exakter Ein-/Ausgangslinearisierung entworfene Regler berücksichtigt das komplette nichtlineare Verhalten des Katalysatormodells, sodass nichts dagegen spricht, allein den Emissionsregler zu nutzen und auf die separate Strategie zu verzichten. Aufgrund der asymptotischen Stabilität des Katalysators ist zu erwarten, dass auch der LQ-Regler mit dieser Aufgabe zurechtkommen kann.

In Bild 6.18 sind die Messverläufe zu diesem Testszenario dargestellt. Im Bild sind die Verläufe der Stellgröße des Lambdaregelkreises, der Spannung $U_{\text {su }}$ der Sprung-Lambdasonde hinter dem Katalysator, der CO- und der NO-Konzentration sowie der drei Zustände des Open-LoopBeobachters dargestellt. Im Vergleich zum ersten Testszenario liegt die Temperatur etwas niedriger, bei $773 \mathrm{~K}$, und die Raumgeschwindigkeit doppelt so hoch, bei $100000 \mathrm{~h}^{-1}$. Am Laborprüfstand ist dieses Szenario so implementiert, dass für eine gewisse Zeit der Stellwert $\lambda_{\mathrm{w}}$ des Emissionsreglers mit dem Lambdawert 1,07 überschrieben wird und ab dem Zeitpunkt $5 \mathrm{~s}$ der richtige Stellwert ausgegeben wird. Wie bisher diskutiert, zeichnet sich der nichtlineare Regler durch die höchste Stellgrößenausnutzung aus. Unabhängig von der Raumgeschwindigkeit bleiben die Zeitkonstante und die Totzeit der Massendurchflussregler konstant. Damit verschärft sich die Problematik mit der Approximation des Totzeitgliedes bei der nun doppelten Raumgeschwindigkeit weiter. Infolgedessen schwingt die Stellgröße stark im Vergleich zu der des LQ-Reglers. Bei der Lambda-Eins-Regelstrategie benötigt die Spannung $U_{\text {su }}$ mehr als $30 \mathrm{~s}$ bis sie in die Nähe der angestrebten $650 \mathrm{mV}$ kommt. Die Emissionsregler sind dabei deutlich schneller, wenn auch die Spannung etwas überschwingt, um $25 \mathrm{mV}$ beim nichtlinearen Regler und um $70 \mathrm{mV}$ beim LQ-Regler. Die Verläufe der Spannung $U_{\text {su }}$, der CO- und der NO-Konzentration sowie der Sauerstofffüllstände des Beobachters sind beim nichtlinearen Regler und beim LQ-Regler sehr ähnlich. Das Entleeren des Sauerstoffspeichers ist mit einem Anstieg der CO-Konzentration verbunden und es ist eine gute Korrelation zwischen der Spannung $U_{\text {su }}$ und der CO-Konzentration zu sehen. Die Abhängigkeit der NO-Konzentration von der Spannung ist jedoch nicht direkt und besteht nur 
Stellgröße des Lambdaregelkreises
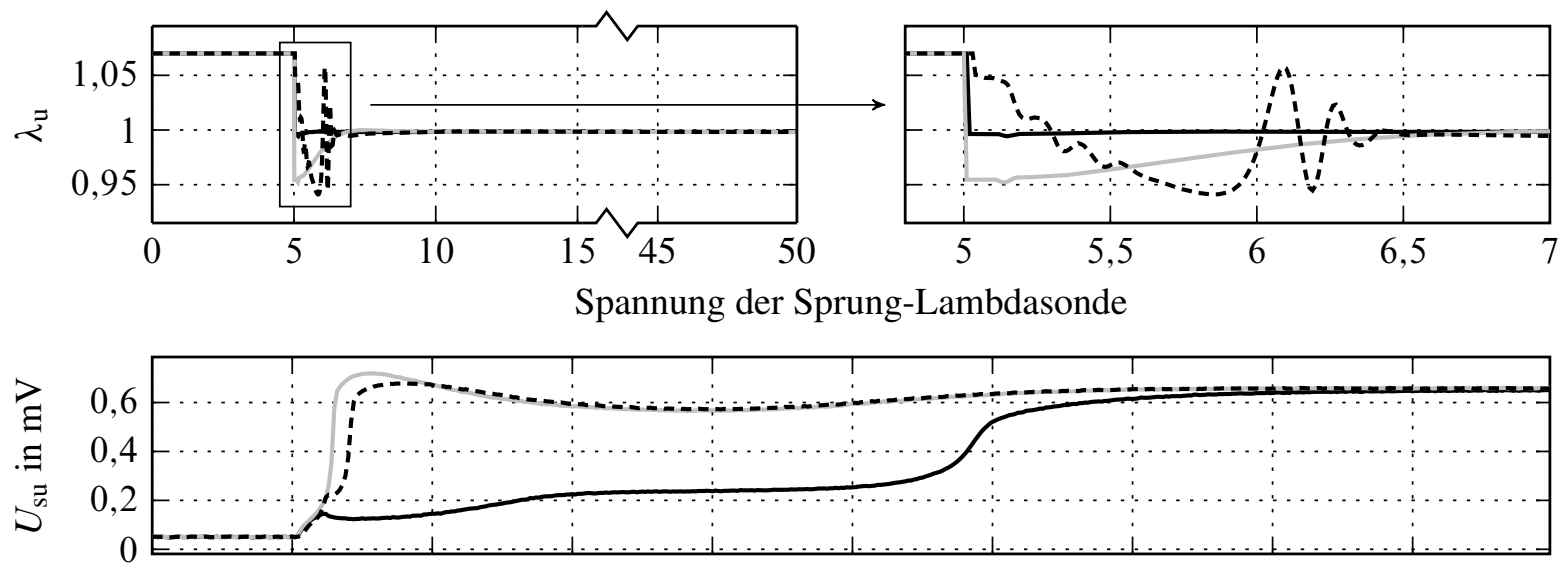

CO- und NO-Konzentrationen
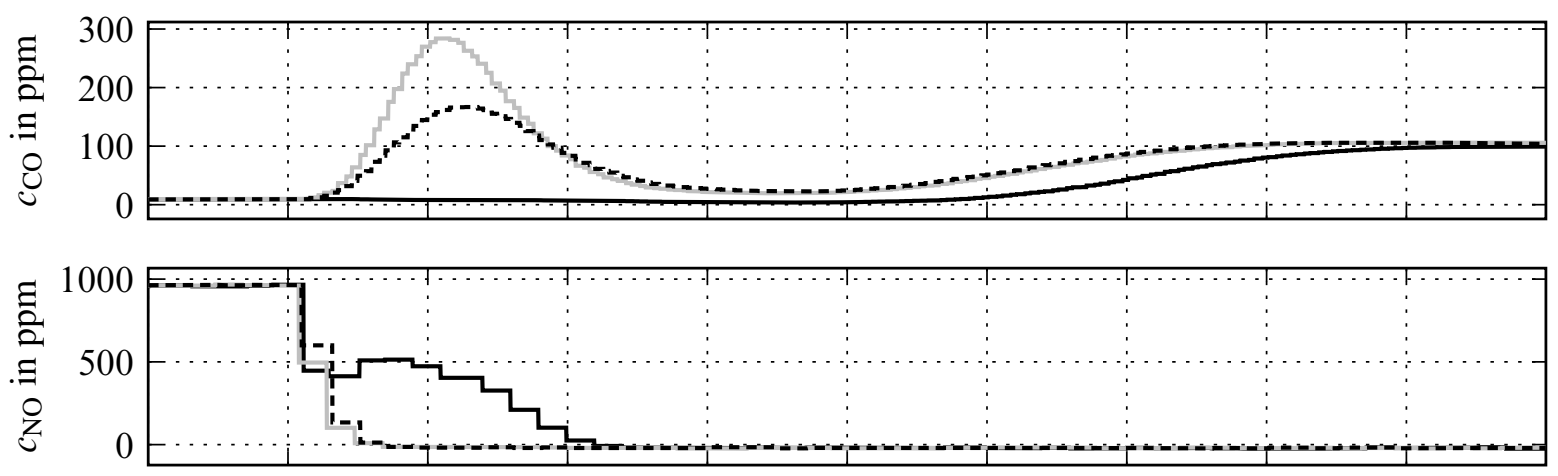

Sauerstofffüllstände der drei Modellzellen
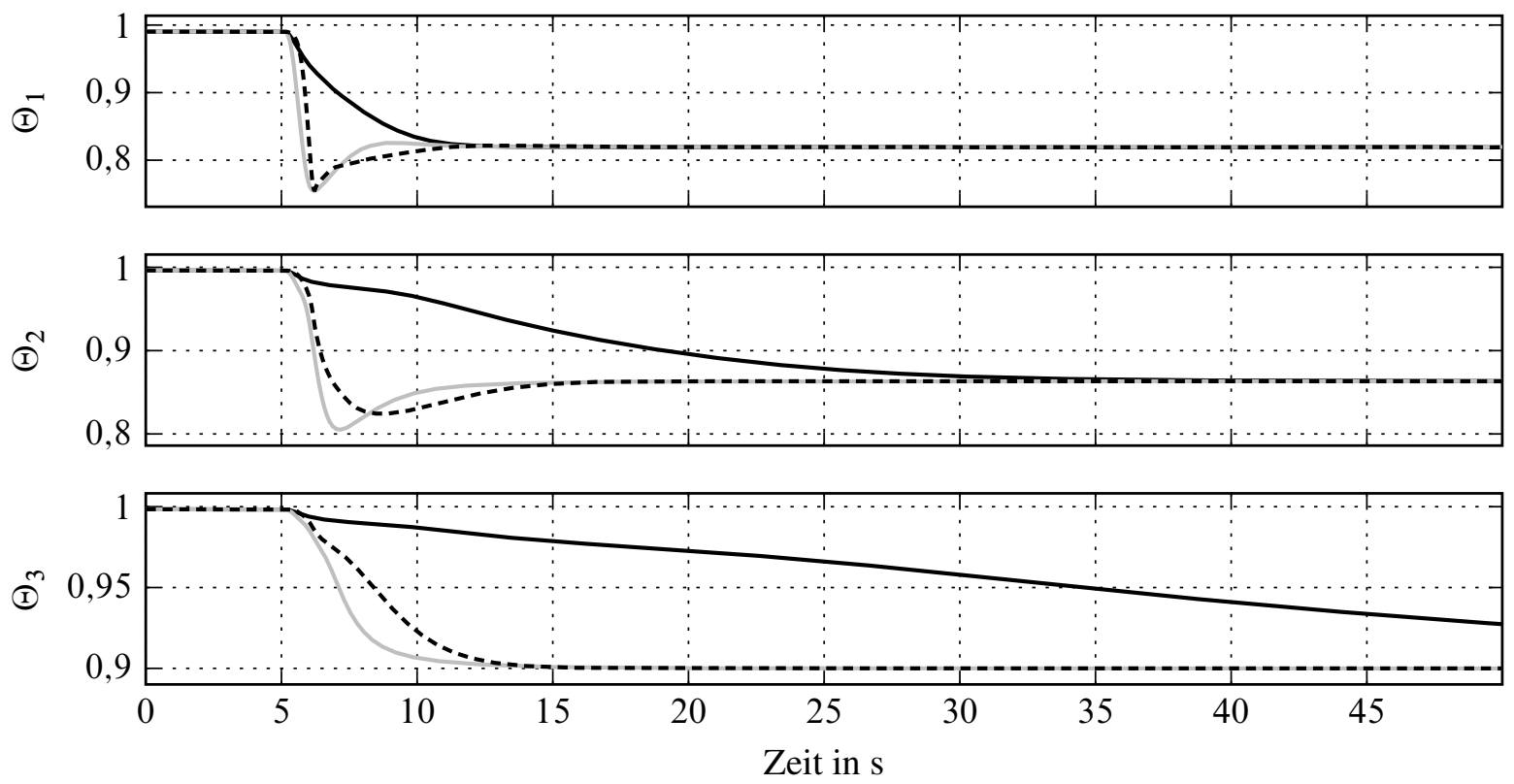

Bild 6.18: Vergleich der Lambda-Eins-Regelstrategie (-), des LQ-Reglers $(-)$ und der exakten Ein-/Ausgangslinearisierung für $\Theta_{3}(---)$ anhand des fuel cut-off Testszenarios. Die Temperatur beträgt $773 \mathrm{~K}$ und die Raumgeschwindigkeit $100000 \mathrm{~h}^{-1}$. 
für Spannungen unter $200 \mathrm{mV}$. Interessanterweise steigt bei der Lambda-Eins-Regelstrategie kurz nach $5 \mathrm{~s}$ die NO-Konzentration wieder an, obwohl die Spannung $U_{\mathrm{su}}$ keinen starken Einbruch aufweist. Das Ansteigen der NO-Konzentration kann mit dem Katalysatormodell nicht erklärt werden, was am fehlenden NO-Reaktionsmechanismus liegt. Weiterhin wurden technische Ursachen in wiederholten Messungen ausgeschlossen und die Messwerte der Massendurchflussregler zeigen keine Anzeichen auf ein unbeabsichtigtes Dosieren von magerem Gasgemisch.

Die Messergebnisse zeigen, dass die Emissionsregler während und nach Phasen mit Unterbrechung der Kraftstoffeinspritzung nicht ausgeschaltet werden müssen und sie die NO-Emissionen reduzieren können. Es ist dennoch sinnvoll, eine Situationserkennung zu entwerfen und die Regler je nach Situation neu zu parametrieren. So können z. B. die erhöhten CO-Emissionen in Bild 6.18 vermieden werden. Der Vorteil gegenüber der separaten Steuerstrategie ist, dass keine Strukturumschaltungen im Reglkreis stattfinden und die damit verbundenen Probleme gar nicht entstehen.

Einfluss der Stellgrößenbeschränkung Die Messungen am Laborprüfstand zeigen, dass erhöhte NO-Emissionen hinter dem Katalysator vor allem dann auftreten, wenn der Sauerstoffspeicher fast vollständig befüllt ist. Von der gezielten Unterbrechung der Kraftstoffeinspritzung in bestimmten Fahrsituationen abgesehen, passiert dies in der Praxis vor allem dann, wenn die Stellgröße des Lambdaregelkreises in die Stellgrößenbeschränkung läuft. Aus diesem Grund wird im letzten Testszenario die Stellgrößenbeschränkung gezielt variiert.

Die eingesetzte synthetische Lambdaabweichung besteht aus Pulsen mit einer Amplitude von $6 \%$, einer Periodendauer von 7,5 Sekunden und einer Pulsweite von 1,5 Sekunden. Die Stellgrößenbeschränkung wird alle zwei Minuten reduziert, von anfänglichen $\pm 7 \%$ auf $\pm 5 \%$ und schließlich auf $\pm 3 \%$. Es werden die Lambda-Eins-Regelstrategie und der LQ-Regler miteinander verglichen. Der mittels exakter Ein-/Ausgangslinearisierung entworfene Regler benötigt hohe Stellgrößenbeträge und kann bei der starken Stellgrößenbeschränkung die nichtlineare Katalysatordynamik nicht wie erwünscht kompensieren. Die Folge daraus sind starke Schwingungen der Stellgröße und teilweise höhere NO-Emissionen im Vergleich zu denen bei der Lambda-Eins-Regelstrategie. Aus diesem Grund wird beim folgenden Vergleich auf die entsprechende Darstellung verzichtet.

In Bild 6.19 sind die Verläufe der Lambdaabweichung, der Stellgröße des Lambdaregelkreises, der Spannung der Sprung-Lambdasonde hinter dem Katalysator, der CO- und der NO-Emissionen sowie der Zustände des Open-Loop-Beobachters dargestellt. Bei der Stellgrößenbeschränkung von $\pm 7 \%$ lässt der LQ-Regler die Spannung $U_{\text {su }}$ nur wenig einbrechen und kann im Vergleich zu der Lambda-Eins-Regelstrategie die NO-Emissionen wirkungsvoll unterdrücken. Die Reduktion beträgt im Vergleich zur Lambda-Eins-Regelstrategie $70 \%$. Die CO-Emissionen schwingen dabei mit einer deutlich niedrigen Amplitude um den Mittelwert von 110 ppm. Interessant an diesem Experiment ist, dass, obwohl die Lambdaabweichung positiv ist, der Sauerstoffspeicher bei der Lambda-Eins-Regelstrategie nicht befüllt sondern geringfügig entleert wird. Die Erklärung dafür ist Folgende: Die Lambda-Zeit-Flächen am Anfang und am Ende jedes Pulses, die aufgrund der Dynamik des Lambdaregelkreises resultieren und am Katalysatoreingang anliegen, sind zwar gleich, jedoch ist der dazugehörige Sauerstoffgehalt im Gas nicht gleich und die Sauerstofffüll- 
a)
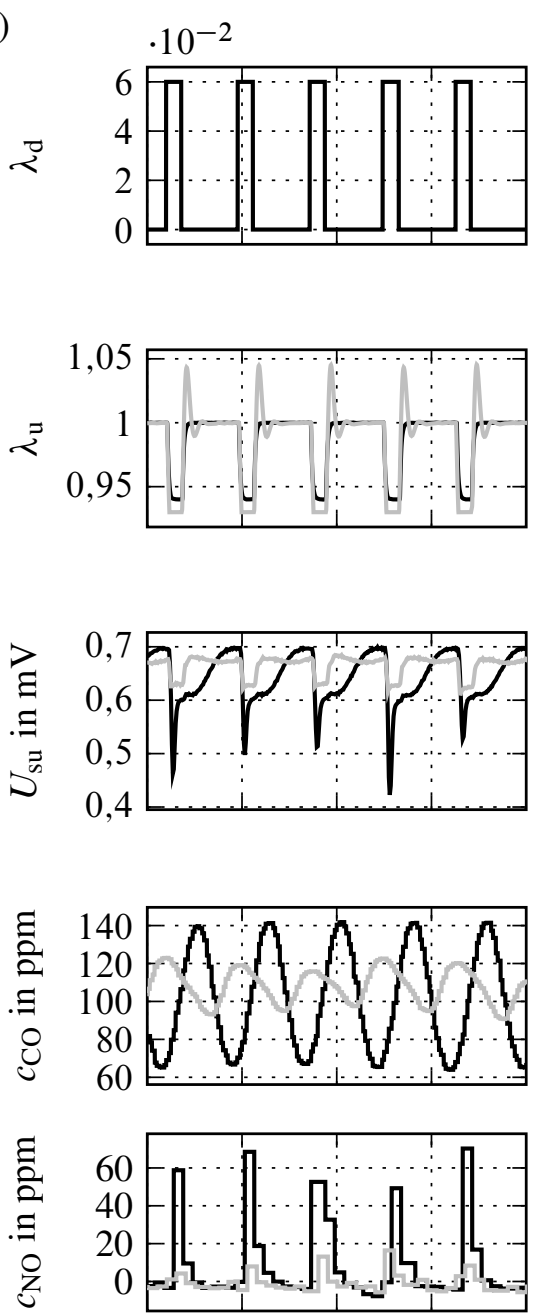

b)

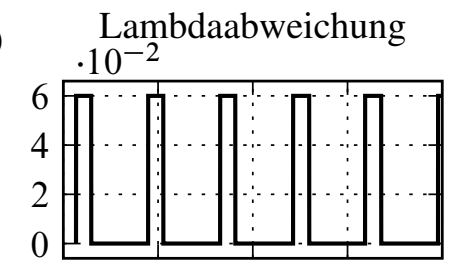

c)

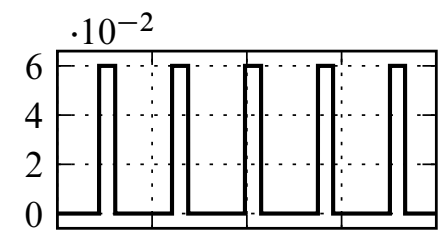

Stellgröße des Lambdaregelkreises
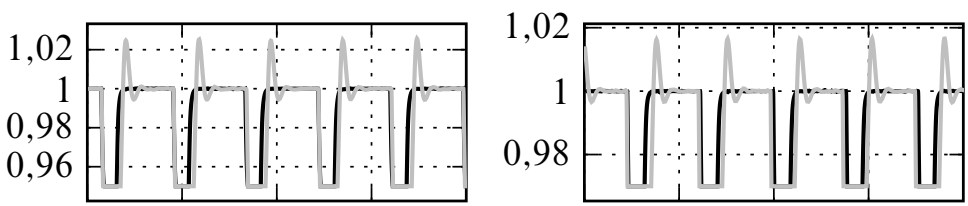

Spannung der Sprung-Lambdasonde
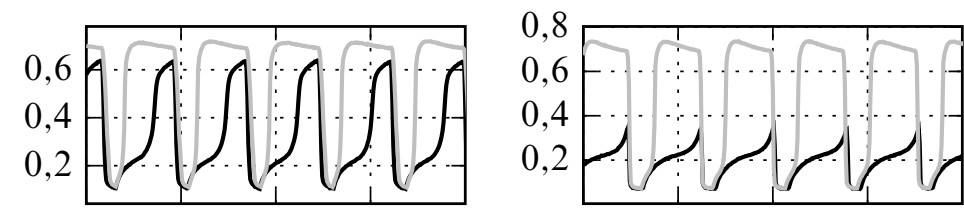

CO- und NO-Konzentrationen
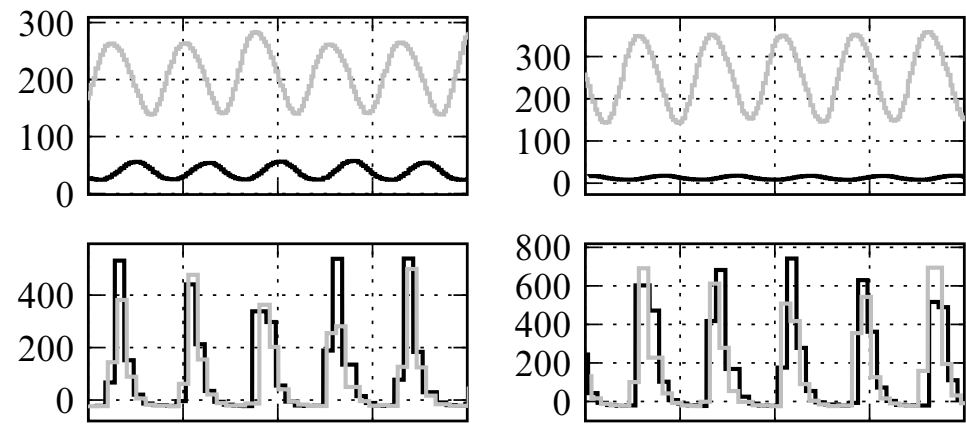

Sauerstofffüllstände der drei Modellzellen
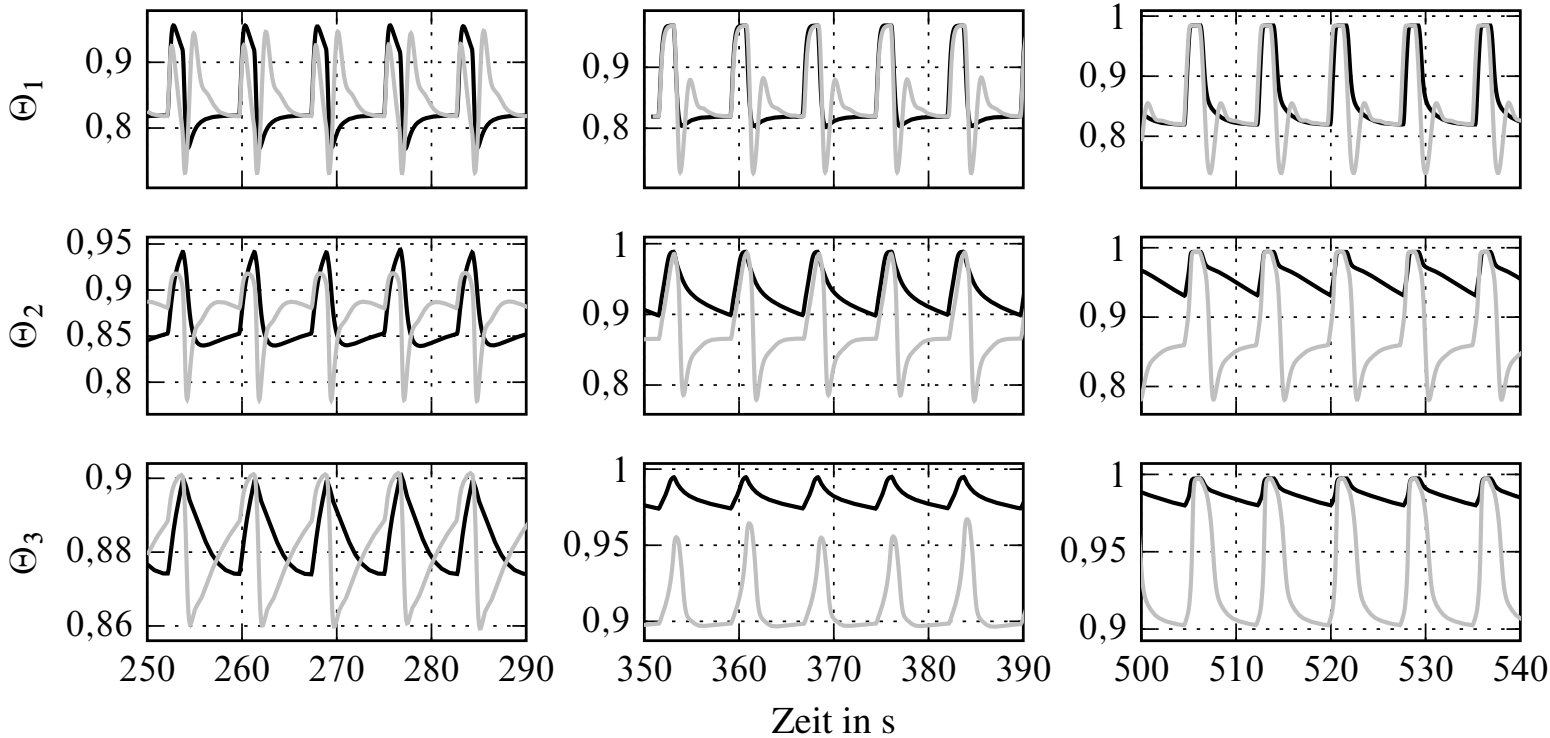

Bild 6.19: Vergleich der Lambda-Eins-Regelstrategie $(-)$ und des LQ-Reglers $(-)$ bei Variation der Stellgrößenbeschränkung des Lambdareglers: a) $\pm 7 \%$; b) $\pm 5 \%$; c) $\pm 3 \%$. Die Temperatur beträgt $773 \mathrm{~K}$ und die Raumgeschwindigkeit $100000 \mathrm{~h}^{-1}$. 
standsskala des Katalysators ist nicht linear. Daraus resultiert die Verringerung des Sauerstofffüllstandes. Bei der Stellgrößenbegrenzung von $\pm 5 \%$ geht die Stellgröße in die Sättigung und die NO-Emissionen steigen an. Dabei korrelieren die NO-Emissionen vor allem mit Spannungen, die unter $200 \mathrm{mV}$ liegen. Der LQ-Regler kann jedoch die Spannung schnell auf den Sollwert bringen und die NO-Emissionen um 15\% im Vergleich zur Lambda-Eins-Regelstrategie reduzieren. Die Sauerstofffüllstände können dabei gut geregelt werden. Die CO-Emissionen sind im Vergleich zum ersten Versuchsabschnitt angestiegen, obwohl nach den Verläufen der Sauerstofffüllstände eher mit einer Verringerung gerechnet werden kann. An dieser Stelle stößt das Modell an seine Grenzen. Im letzten Versuchsabschnitt wird die Stellgröße noch stärker begrenzt. Der LQ-Regler kann nach wie vor die Spannung $U_{\text {su }}$ schnell auf den Sollwert bringen und kann bei leicht angestiegenen CO-Emissionen die NO-Emissionen im Vergleich zu der Lambda-Eins-Regelstrategie um $12 \%$ reduzieren. An den letzten zwei Versuchsabschnitten ist gut zu erkennen, dass, wie weiter oben erwähnt, die Spannung der Sprung-Lambdasonde alleine nicht als Maß für die Regelgüte geeignet ist. So lässt z. B. der Verlauf von $U_{\text {su }}$ im dritten Versuchsabschnitt eine deutlich bessere Regelgüte beim LQ-Regler im Vergleich zu der Lambda-Eins-Regelstrategie vermuten, was mit den NO-Konzentrationsverläufen nicht bestätigt werden kann.

\subsection{Zusammenfassung}

Der Gegenstand dieses Kapitels ist die Vorstellung eines Regelungskonzeptes für den Katalysator und der Entwurf und der Vergleich von verschiedenen Reglern. Geleitet von den Ergebnissen der Systemanalyse wird ein Open-Loop-Beobachter für den Sauerstofffüllstand des Katalysators eingesetzt und es werden auch nichtlineare Regler entworfen. Die entworfenen Regler werden je nach Regelziel in zwei Gruppen aufgeteilt: Regler für den Sauerstofffüllstand und Regler, deren Regelziel sich aus einer Betrachtung der Konvertierungsraten der Spezies ableitet. Der Reglerentwurf mittels exakter Ein-/Ausgangslinearisierung ist erst nach einer minimalphasigen Approximation der Totzeit im Lambdaregelkreis möglich. Obwohl die entworfenen Regler eine hohe Regelgüte aufweisen, resultiert die Kompensation der Systemnichtlinearitäten zusammen mit der $\mathrm{PT}_{1}$-Approximation des Totzeitgliedes in hohen Stellgrößenbeträgen. Der hohe Stellgrößenaufwand und die gleichzeitig strikte Stellgrößenbeschränkung resultieren in bestimmten Situationen in Schwingungen der Stellgröße und teilweise in erhöhten Schadstoffemissionen. Auf der anderen Seite wird ein LQ-Reglerentwurf ähnlich wie in der Literatur durchgeführt, allerdings mit dem Unterschied einer modellbasierten Auslegung der Gewichtungsmatrix der Modellzustände im Gütemaß. Der LQ-Regler zeichnet sich in den Testszenarien durch eine hohe Regelgüte bezogen auf die Konvertierungsraten der Gasspezies aus. Aufgrund der starken Arbeitspunktabhängigkeit des Katalysators können jedoch auch Testszenarien konstruiert werden, in denen der lineare LQ-Regler keine nennenswerten Vorteile bei der Emissionsreduktion gegenüber einer klassischen Lambda-Eins-Regelstrategie erzielt. Es ist daher empfehlenswert, in einer Nachfolgearbeit zu untersuchen, inwieweit diese Testszenarien in der Praxis auftreten können. Als nächster Schritt beim LQ-Reglerentwurf kann versucht werden, die Totzeit im Lambdaregelkreis beim Reglerentwurf zu berücksichtigen. Der LQ-Regler dient als Basis für die Implementierung einer nichtlinearen 
modellprädiktiven Regelung, die zwar nicht echtzeitfähig implementiert wird, jedoch gut als Referenz für die anderen Regelungsverfahren eingesetzt werden kann. Die aktuelle Auslegung zeigt keine nennenswerten Vorteile gegenüber dem LQ-Regler. Es stehen aber viele neue Möglichkeiten beim Entwurf offen, die in weiterführenden Arbeiten untersucht werden können. 


\section{Zusammenfassung und Ausblick}

Die drei Ziele dieser Arbeit sind die Entwicklung von nichtlinearen Zustandsraummodellen des Katalysators aus vereinfachten physikochemischen Modellen, eine systemtheoretische Analyse des Katalysators und das Aufstellen eines geeigneten Regelungskonzeptes für seine Regelung. Die Ergebnisse in dieser Arbeit entstanden im Rahmen des Projektes „ReffKat“, das unter der Federführung der Firma Umicore durchgeführt worden ist und ein Teil der Förderinitiative „MatRessource“ des Bundesministeriums für Bildung und Forschung darstellt. Das neu entwickelte nichtlineare Zustandsraummodell des Katalysators und das entwickelte Regelungskonzept sind an einem Laborprüfstand der Firma Umicore implementiert und verifiziert worden.

Das Erreichen der gestellten Ziele erfordert zuerst eine Einführung in das Gebiet der Abgasnachbehandlung, die in Kapitel 1 erfolgt. Nach einem kurzen Überblick über die Geschichte der Abgasnachbehandlung, den Aufbau des Katalysators sowie die heutigen Randbedingungen beim Ressourceneinsatz für die Katalysatorherstellung werden die Notwendigkeit des Einsatzes modellbasierter Regelungsverfahren und die Struktur dieser Arbeit erläutert.

Der Regelkreis zur Abgasnachbehandlung weist eine Kaskaden-Struktur auf, die aus einer unterlagerten Lambdaregelung und einer überlagerten Emissionsregelung besteht. Das Ziel dieser Arbeit ist zwar der Entwurf des Emissionsreglers, jedoch wird dafür auch der unterlagerte Lambdaregelkreis benötigt. Aus diesem Grund widmet sich Kapitel 2 dem Motormodell und dem Entwurf eines Lambdareglers. Nach einer kurzen Übersicht über die Modellierung der wichtigsten Motorkomponenten werden die Anforderungen an den Lambdaregler anhand von vorliegenden, realen Messungen an einem Motorprüfstand festgelegt. Aufgrund der Vorteile beim Reglerentwurf und bei der Reglerimplementierung wird die IMC-Struktur eingesetzt. Der entworfene, adaptive, diskrete Lambdaregler kann die gewünschte Bandbreite bei höheren Motordrehzahlen und Motorlastmomenten erreichen, während die Totzeit und die strikte Stellgrößenbegrenzung die Bandbreite bei niedrigen Motordrehzahlen und Motorlastmomenten reduzieren, was den Einsatz des Katalysators notwendig macht.

Den Ausgangspunkt eines jeden modellbasierten Reglerentwurfs stellt ein Systemmodell dar, das zum einen die wesentliche Systemdynamik wiedergibt und zum anderen möglichst einfach und transparent ist. In der Literatur sind sowohl physikochemische als auch phänomenologische Katalysatormodelle zu finden. Jedoch liegen die meisten Modelle nicht in Form von Zustandsraummodellen vor, was die systemtheoretische Analyse des Katalysators erschwert, denn viele Methoden der Regelungstechnik gehen von dieser Systemdarstellung aus. Aus diesem Grund wird in Kapitel 3 aus einem vereinfachten physikochemischen Modell für die Sauerstoffspeicherung durch Ceroxid ein Zustandsraummodell erstellt. Interessanterweise kann das komplette Modell alleine durch fünf Funktionen beschrieben werden, die in dieser Arbeit Gewichtsfunktionen genannt werden. Die Vorgehensweise zu ihrer Herleitung wird auch auf andere als das in der Arbeit ver- 
wendete Reaktionsschema übertragen und es werden beispielhaft zwei andere Reaktionsschemata durch Gewichtsfunktionen beschrieben.

Die neuartigen Gewichtsfunktionen beschreiben das Katalysatorverhalten einerseits quantitativ, sind andererseits aber auch sehr anschaulich. Ihre Werte hängen mit der Fähigkeit der einzelnen Gasspezies zusammen, den Sauerstoffspeicher zu befüllen oder zu entleeren. In Kapitel 4 werden die Gewichsfuktionen aus systemtheoretischer und aus chemischer Sicht analysiert. Aus systemtheoretischer Sicht bieten sie die vorteilhafte Nutzung der vorhandenen Systemstruktur, um nichtlineare, in den Eingangskonzentrationen lineare Zustandsraummodelle zu erstellen. Die Modelldarstellung im Zustandsraum ermöglicht z. B. den analytischen Nachweis der asymptotischen Stabilität des Katalysatormodells und seine analytische Linearisierung. Das linearisierte Modell bildet die Basis zur Berechnung von Strukturmaßen, die den quantitativen Vergleich von verschiedenen Katalysatoren ermöglichen. Zum Beispiel kann die aus der Literatur bekannte schlechte Beobachtbarkeit des Katalysators im Bereich des Lambdafensters mit dem Beobachbarkeitmaß nach Lückel/Müller belegt werden. Weiterhin wird der Einfluss der Temperatur und des Massenstroms des Motorabgases auf die zuvor berechneten Systemeigenschaften analysiert. Eine wichtige Erkenntnis dabei ist, dass Ressourceneinsparungen bei der Katalysatorherstellung und ein gutes Regelverhalten keine konkurrierenden Ziele sind.

Um das Katalysatormodell in der Praxis einsetzen zu können, werden in Kapitel 5 seine Parameter optimiert. Von den Projektpartnern werden bereits gute Parameterstartwerte zur Verfügung gestellt, sodass die Optimierung nur in kleinen Bereichen stattfindet. Der eigentlichen Optimierung geht eine analytische Sensitivitätsanalyse voraus, die die für das Ein-/Ausgangsverhalten wesentlichen Parameter aufzeigt: die thermodynamischen Parameter von Ceroxid. Die festgelegte Identifikationstrajektorie wird am Laborprüfstand nachgefahren und mit den gewonnenen Messdaten werden die gewählten Modellparameter mit einem genetischen Algorithmus optimiert. Für die Verifikation der Parameter wird eine der Identifikationstrajektorie ähnliche Lambdatrajektorie eingesetzt, allerdings bei einer niedrigeren und einer höheren Raumgeschwindigkeit.

Kapitel 6 widmet sich der letzten Aufgabe dieser Arbeit, der Regelung des Katalysators. Seine schlechte Beobachtbarkeit und seine asymptotische Stabilität motivieren den Einsatz eines OpenLoop-Beobachters, während sein stark nichtlineares Verhalten den Entwurf von nichtlinearen Reglern motiviert. Der Reglerentwurf mittels exakter Ein-/Ausgangslinearisierung ist erst nach einer minimalphasigen Approximation des Totzeitgliedes im Lambdaregelkreis möglich. Die entworfenen Regler zeigen eine sehr hohe Regelgüte, die allerdings mit einem hohen Stellaufwand verbunden ist, der in der Praxis nicht zu realisieren ist. Da nur eine Stellgröße, der Lambdawert am Katalysatoreingang, zur Verfügung steht, kann auch nur eine Regelgröße gewählt werden. Dies macht es weiterhin schwierig, die Konvertierung aller Gasspezies gleichermaßen zu berücksichtigen. Einen Ausweg stellt der Entwurf eines LQ-Reglers dar, bei dem die Gewichtungsmatrix der Zustände im Gütemaß die Sensitivitäten der $\mathrm{O}_{2-}$, der $\mathrm{CO}$ - und der $\mathrm{H}_{2}$-Konvertierungsrate widerspiegelt. Bezogen auf die Konvertierungsraten erzielt der LQ-Regler die höchste Regelgüte, zeichnet sich durch einen geringen Stellgrößenaufwand aus und ist gleichzeitig der am einfachsten zu implementierende Regler. Mit demselben Gütemaß wird eine nichtlineare modellprädiktive Regelung implementiert, die als Referenz für die anderen Regler gedacht ist. 
Das in dieser Arbeit vorgestellte Vorgehen bei der Modellbildung, der Systemanalyse und dem Reglerentwurf bezieht sich zwar auf einen Drei-Wege-Katalysator, ist jedoch nicht auf ihn beschränkt. Solange die hier gemachten Annahmen zulässig und die gestellten Voraussetzungen erfüllt sind, können auch andere Katalysatortypen oder chemische Systeme auf diese Weise modelliert, analysiert und geregelt werden.

Ausblick Die Ergebnisse in dieser Arbeit können zwar mit wenig Aufwand unmittelbar in der Praxis eingesetzt werden, es sind jedoch einige Punkte vorhanden, die in weiterführenden Arbeiten zu untersuchen sind.

Das verwendete Reaktionsschema enthält wichtige Schadstoffe wie z. B. NO und $\mathrm{C}_{3} \mathrm{H}_{6}$ nicht, sodass eine Umrechnung in eine äquivalente $\mathrm{O}_{2-}$, CO- oder $\mathrm{H}_{2}$-Konzentration vorgenommen wird. Im Lauf des Projektes ist zum einen durch die eigenen Messungen am Laborprüfstand und zum anderen durch die Arbeiten der Projektpartner festgestellt worden, dass die Korrelation zwischen den NO- und den $\mathrm{O}_{2}$-Emissionen doch nicht so direkt wie angenommen ist. Daher ist es sinnvoll, die Reaktionsmechanismen weiter zu studieren und zu versuchen, alle limitierten Schadstoffe im Katalysatormodell zu berücksichtigen. Solche Modelle sind zwar schon heute bekannt, jedoch gehören sie zu der Gruppe der detaillierten physikochemischen Modelle und sind daher zu komplex zum Reglerentwurf. Die Herausforderung ist es, die Reaktionsmechanismen so zu berücksichtigen, dass eine Darstellung mittels Gewichtsfunktionen möglich ist und damit die Ergebnisse dieser Arbeit direkt auf das neue Reaktionsschema übertragen werden können.

Um das Regelungskonzept zu verifizieren, werden in dieser Arbeit vorwiegend synthetische Testszenarien verwendet. Diese bringen die Regler an ihre Grenzen und erleichtern das Aufzeigen von bestimmten Sachverhalten, besitzen aber nicht immer ein Äquivalent in der Praxis. Basierend auf Messungen an realen Fahrzeugen oder Motorprüfständen sollten die Fahrsituationen identifiziert werden, die mit erhöhten Schadstoffemissionen verbunden sind. Anschließend sollten die Regler in der Simulationsumgebung und am Laborprüfstand mit diesen Testszenarien getestet und optimiert werden. An den gewonnenen Testszenarien sollte die Beobachtbarkeit des Katalysators im realen Betrieb untersucht und ein Algorithmus zur Anpassung der Modellparameter erarbeitet werden. Der nächste Schritt wäre die Implementierung des Regelungskonzeptes ins Motorsteuergerät, was eine enge Zusammenarbeit mit den Fahrzeugherstellern notwendig machen würde.

Simulationsstudien in dieser Arbeit zeigen, dass mit abnehmender Totzeit im Lambdaregelkreis die Stellgrößenausnutzung der mittels exakter Ein-/Ausgangslinearisierung entworfenen Regler abnimmt. Bei SCR-Katalysatoren sind die Totzeiten klein, da die Harnstofflösung vor dem Katalysator eingespritzt wird und die Totzeit des Motors keine Rolle spielt. In Abschnitt 3.3 wird erläutert, dass das Reaktionsschema eines $\mathrm{NH}_{3}$-Oxidationskatalysators, der oft mit einem SCRKatalysator kombiniert wird, ebenfalls durch Gewichtsfunktionen dargestellt werden kann. Beim SCR-Katalysator kann daher eine inversionsbasierte Methode ihre Vorteile eventuell besser ausspielen. Dieses Reaktionsschema zeigt auch, dass die hier erarbeiteten Methoden nicht nur auf Drei-Wege-Autoabgaskatalysatoren beschränkt sind und ihre Anwendung an weiteren chemischen Systemen untersucht werden sollte. 


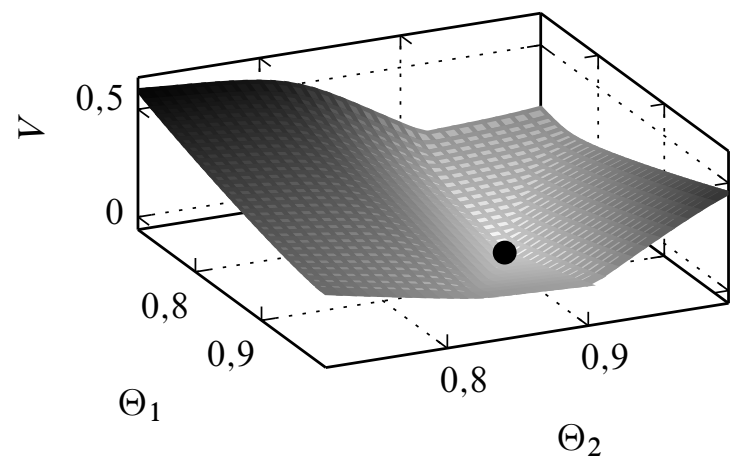

Bild 7.1: Beispiel für eine numerisch generierte Ljapunov-Funktion. Hierbei lautet die Funktion $f$ in Abhängigkeit der durchlaufenen Trajektorienlänge $l: f=e^{l}-1$.

Der LQ-Regler eignet sich gut zur Regelung des Katalysators, es können jedoch auch Testszenarien konstruiert werden, bei denen der LQ-Regler nur vergleichbar mit einer klassischen LambdaEins-Regelstrategie abschneidet. Es wäre interessant, auf Basis des LQ-Reglers einen nichtlinearen Regler zu entwerfen, der in bestimmten Bereichen ein besseres Verhalten als der lineare Regler aufweist. Der Reglerentwurf könnte z. B. auf Basis einer Control-Ljapunov-Funktion erfolgen [2]. Eine erste solche Ljapunov-Funktion kann numerisch aus den Zustandstrajektorien des mit dem LQ-Regler geregelten Katalysators gewonnen werden. Zum Beispiel können die Zustandstrajektorien ab der Ruhelage rückwärts durchlaufen werden und dabei jeweils eine streng monoton steigende Funktion in Abhängigkeit der durchlaufenen Trajektorienlänge definiert werden. Die Endpunkte dieser Funktionen liegen auf einer Ljapunov-Funktion des Systems, die für den Reglerentwurf zuerst gezielt modifiziert und analytisch approximiert werden könnte, siehe Bild 7.1.

Nicht zuletzt ist zu erwähnen, dass Hybridantriebe ein sehr großes Potential zur Reduktion der Schadstoffemissionen haben. Denn nicht nur durch den geringeren Kraftstoffverbrauch werden die Schadstoffemissionen reduziert, sondern auch durch den gleichmäßigeren Betrieb des Verbrennungsmotors. Die hochdynamischen Fahrlasten können vom zweiten, oft elektrischen Leistungszweig abgefangen und der Motorbetriebspunkt kann langsam und ohne große Lambdaabweichungen an die neue Leistungsanforderung angepasst werden, was als die sogenannte Phlegmatisierung des Verbrennungsmotors bezeichnet wird [42]. Aufgrund des gleichmäßigeren Betriebs des Verbennungsmotors kann des Weiteren die Sauerstoffspeicherfähigkeit des Katalysators reduziert werden und auf dieses Weise können Ressourcen eingespart werden. 


\section{Anhang}

\section{A.1 Parameter der Motorarbeitspunkte}

Für die Simulationen des Lambdaregelkreises und des Katalysatormodells werden je nach Anforderung drei verschiedene Motorarbeitspunkte genutzt. Diese sind von realen Messdaten an einem Motorprüfstand abgeleitet, siehe die Arbeit von Möller [64], und repräsentieren die Verhältnisse am Motor und am Katalysator bei niedriger, mittlerer und hoher Motorlast. In Tabelle A.1 sind die Parameter des Motormodells, die Zeitkonstanten und die Totzeit, sowie die Temperatur und die Raumgeschwindigkeit des Motorabgases angegeben. Die Temperaturen $T_{\mathrm{g}}^{\mathrm{in}}, T_{\mathrm{g}}$ und $T_{\mathrm{s}}$ werden gleichgesetzt. Die angegebenen Raumgeschwindigkeiten beziehen sich auf Normalbedingungen, d. h. eine Temperatur von 273,15 K und ein Druck von 1013,25 hPa.

Tabelle A.1: In der Simulationsumgebung eingesetzte Motorarbeitspunkte

\begin{tabular}{lllll}
\hline \multirow{2}{*}{$\begin{array}{l}\text { Nr. } \\
\text { Arbeitspunkt }\end{array}$} & \multicolumn{2}{c}{ Zotormodell } & \multicolumn{2}{c}{ Motorabgas } \\
& Zeitkonstante & Totzeit & Temperatur & Raumgeschwindigkeit \\
\hline 1 & $0,36 \mathrm{~s}$ & $0,28 \mathrm{~s}$ & $700 \mathrm{~K}$ & $16900 \mathrm{~h}^{-1}$ \\
2 & $0,12 \mathrm{~s}$ & $0,13 \mathrm{~s}$ & $850 \mathrm{~K}$ & $24600 \mathrm{~h}^{-1}$ \\
3 & $0,04 \mathrm{~s}$ & $0,05 \mathrm{~s}$ & $920 \mathrm{~K}$ & $70800 \mathrm{~h}^{-1}$ \\
\hline
\end{tabular}

\section{A.2 Laborprüfstand}

Der Laborprüfstand stellt eine Modellgasanlage dar, an der Katalysator-Bohrkerne mit einem Durchmesser von 1 " und einer Länge zwischen 1 " und 3 " unter reproduzierbaren Bedingungen vermessen werden können. In [11] ist der Aufbau des Laborprüfstandes detailliert beschrieben und in Bild A.2 ist sein prinzipieller Aufbau dargestellt. Das Gasgemisch vor dem Katalysator wird von Massendurchflussreglern dosiert und setzt sich aus einem statischen und einem dynamischen Gasanteil zusammen. Der statische Gasanteil macht den größeren Anteil des Volumenstroms aus und wird durch ein Heizelement geleitet. Der dynamische Gasanteil ermöglicht die schnelle Variation des Lambdawertes. Er wird zwecks Verkürzung der Totzeiten erst hinter dem Heizelement dem statischen Gasanteil hinzugefügt. Die Gasspezies und die dazugehörigen Konzentrationen bzw. Konzentrationsbereiche der beiden Gasanteile sind Tabelle A.2 zu entnehmen. Für das Inertgas $\mathrm{N}_{2}$ wird nicht der Konzentrationsbereich angegeben, sondern die Vorschrift zu Berechnung der Konzentration aus den Konzentrationen $c_{i}$ der restlichen Gasspezies. 


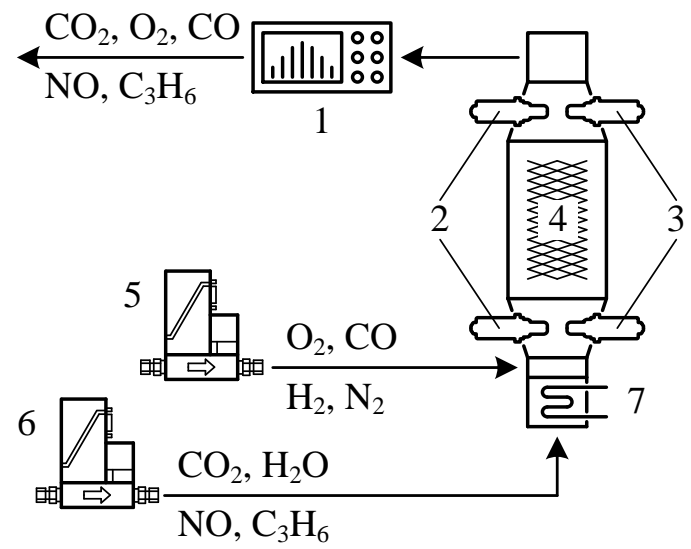

Bild A.2: Prinzipieller Aufbau des Laborprüfstandes: (1) Gasanalysatoren, (2) Sprung- und (3) Breitband-Lambdasonden, (4) Katalysator-Bohrkern, (5) dynamisch und (6) statisch betriebene Massendurchflussregler, (7) Heizelement

Tabelle A.2: Konzentrationen der Gasspezies am Laborprüfstand

\begin{tabular}{llll}
\hline \multicolumn{2}{c}{ statischer Gasanteil } & \multicolumn{2}{c}{ dynamischer Gasanteil } \\
Spezies & Konzentration & Spezies & Konzentrationsbereich \\
\hline $\mathrm{CO}_{2}$ & $13 \%$ & $\mathrm{O}_{2}$ & $0,36-2,46 \%$ \\
$\mathrm{H}_{2} \mathrm{O}$ & $10 \%$ & $\mathrm{CO}$ & $0,28-3,29 \%$ \\
$\mathrm{NO}$ & $1000 \mathrm{ppm}$ & $\mathrm{H}_{2}$ & $0.09-1,1 \%$ \\
$\mathrm{C}_{3} \mathrm{H}_{6}$ & $1000 \mathrm{ppm}$ & $\mathrm{N}_{2}$ & $100 \%-\sum c_{i}$ \\
\hline
\end{tabular}

Die Konzentrationen im statischen Gasanteil und die Konzentrationsabhängigkeit vom Lambdawert im dynamischen Gasanteil orientieren sich dabei an den an einem Motorprüfstand gemessenen Konzentrationsverläufen in Bild 3.4. Diese Ansteuerung der dynamischen Massendurchflussregler zeichnet sich dadurch aus, dass die Sollwerte der Massendurchflussregler stetig über den ganzen Lambdabereich von 0,92 bis 1,08 verändert werden. Im Gegensatz dazu werden bei konventionellen Strategien im Lambdabereich unter eins nur die $\mathrm{CO}$ - und die $\mathrm{H}_{2}$-Gaskonzentration und im Lambdabereich über eins nur die $\mathrm{O}_{2}$-Gaskonzentration variiert, was eine Unstetigkeit des Emissionsmodells bei $\lambda=1$ mit sich bringt.

Messtechnik Zur Erfassung der Lambdawerte sind am Laborprüfstand jeweils eine Breitbandund eine Sprung-Lambdasonde vor und hinter dem Katalysator angebracht. Über Analysegeräten können die $\mathrm{CO}_{2-}, \mathrm{O}_{2^{-}}, \mathrm{CO}-$, $\mathrm{NO}-$ und $\mathrm{C}_{3} \mathrm{H}_{6}$-Konzentrationen gemessen werden. Aufgrund der langen Gaslaufzeiten von der Gasentnahmestelle hinter dem Katalysator zu den Analysegeräten, der niedrigen Abtastraten sowie der Querempfindlichkeiten der Analysegeräte werden die gemessenen Konzentrationen nur für qualitative Vergleiche herangezogen. 


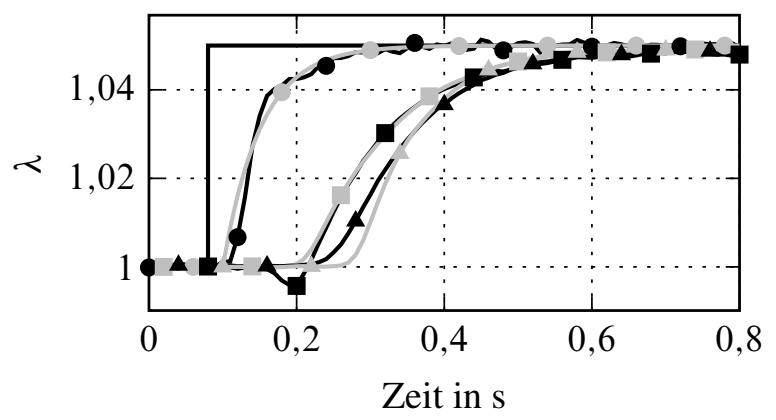

Bild A.3: Gemessene (schwarze Symbole) und simulierte (graue Symbole) Sprungantwort des Laborprüfstandes: Soll-Lambdawert (-), Lambdawert hinter den Massendruchflussreglern $(\rightarrow-,-)$, Lambdawert der Breitband-Lambdasonde vor dem Katalysator $(\rightarrow-,-\rightarrow)$, Lambdawert der Breitband-Lambdasonde hinter dem Katalysator $(\rightarrow,-\rightarrow)$

Dynamisches Modell Am Laborprüfstand ersetzen die Massendurchflussregler zur Dosierung des dynamischen Gasanteils den realen Verbrennungsmotor. Daher wird der in Kapitel 2 für das Motormodell durchgeführte Reglerentwurf nun für die Massendurchflussregler durchgeführt. Ein Massendurchflussregler besteht aus einem Proportionalventil und einem Massendurchflusssensor. Er wird intern über einen PID-Regler geregelt, sodass sich der geschlossene Regelkreis wie ein $\mathrm{PT}_{1}$-Glied mit Totzeit verhält. Das heißt, die beim Entwurf des Lambdareglers zugrunde gelegte Modellstruktur bleibt erhalten. Über die Datenschnittstelle des Massendurchflussregles kann auf den Messwert des internen Sensors zugegriffen werden und dieser kann für eine Parameteridentifikation des Modells genutzt werden. In Bild A.3 ist beispielhaft die Sprungantwort des Laborprüfstandes für einen Lambdasprung von 1 auf 1,05 dargestellt. Aus den Messwerten der Massendurchflusssensoren wird ein Lambdawert berechnet und man kann gut erkennen, dass ein $\mathrm{PT}_{1}$-Glied mit einer Zeitkonstante von $43 \mathrm{~ms}$ und einer Totzeit von $20 \mathrm{~ms}$ die Messung gut wiedergeben kann. Des Weiteren sind in Bild A.3 noch die Messwerte der Breitband-Lambdasonden vor und hinter dem Katalysator und die dazugehörigen Simulationsverläufe dargestellt. Die Lambdasonden werden entsprechend Kapitel 2 jeweils als ein weiteres PT $_{1}$-Glied mit Totzeit modelliert. Ihre Zeitkonstante liegt bei etwa $150 \mathrm{~ms}$, die Transportzeit des Gases ab den Massendurchflussreglern bis zur Lambdasonde vor dem Katalysator liegt bei $180 \mathrm{~ms}$ und es vergehen weitere $70 \mathrm{~ms}$ bis das Gas die Lambdasonde hinter dem Katalysator erreicht. Die Zeitkonstanten und die Transportzeiten sind vom Volumenstrom abhängig und daher wird dieser bei allen Messungen konstant gehalten. Eine Variation der Raumgeschwindigkeit wird durch den Einsatz unterschiedlich großer Katalysator-Bohrkerne erreicht. Es stehen die vier Raumgeschwindigkeiten 50 000, 75 000, 100000 und $150000 \mathrm{~h}^{-1}$ zur Verfügung.

Um hochdynamische Fahrzyklen in Zukunft am Laborprüfstand nachzufahren, wird in [116] die Optimierung der Reglerstruktur der Massendurchflussregler untersucht, in [119] wird die Hysteresekompensation eines Proportionalventils durchgeführt und in [120] wird ein IMC-Regler für die Temperaturregelung des Laborprüfstandes entworfen. 
Vorkonditionierung Um reproduzierbare Messergebnisse zu erzielen, enthält jede Messung vor dem eigentlichen Messabschnitt eine Vorkonditionierung mit fettem Gasgemisch [72]. Zusätzlich werden am Anfang jedes Versuchstages die Kennlinien der Breitband-Lambdasonden kalibriert.

\section{A.3 Regularität der Matrix A}

Eine Voraussetzung für die Berechnung der Konzentrationen in der Gasphase und im Washcoat mit der Gleichung

$$
\left(\begin{array}{l}
\mathbf{c} \\
\mathbf{x}
\end{array}\right)=\underbrace{-\mathbf{A}^{-1}(\Theta) \mathbf{B}}_{\mathbf{G}_{\mathrm{c}, \mathrm{x}}} \mathbf{c}^{\mathrm{in}}
$$

ist die Regularität der Matrix A. Die Inverse einer Matrix kann symbolisch mit dem Gauß-JordanAlgorithmus berechnet werden. Der Algorithmus basiert auf dem Durchführen von elementaren Zeilenumformungen und dabei wird das erste von Null verschiedene Element einer Zeile als Pivotelement bezeichnet [108]. Eine Voraussetzung für die Invertierbarkeit einer Matrix ist, dass in jedem Schritt des Invertierungsalgorithmus ein Pivotelement definiert werden kann. Bei der symbolischen Invertierung der Matrix A wird das erste Element, das nicht identisch Null ist, als Pivotelement definiert. Deshalb ist es notwendig zu untersuchen, ob die gewählten Pivotelemente nach Einsetzen der Modellparameter tatsächlich ungleich Null sind.

Bei der Invertierung der 10×10-dimensionalen Matrix A werden 10 Pivotelemente definiert, wobei die ersten fünf davon folgende Struktur aufweisen

$$
\sigma_{i}=-\underbrace{B_{2, i}}_{>0}-\underbrace{B_{1} T_{\mathrm{g}}}_{>0}
$$

mit $i \in\left\{\mathrm{CO}_{2}, \mathrm{H}_{2} \mathrm{O}, \mathrm{O}_{2}, \mathrm{CO}, \mathrm{H}_{2}\right\}$. Die Struktur dieser Pivotelemente ist unabhängig vom konkreten Reaktionsschema, da sie nur von Massentransportprozessen abhängen. Unter Berücksichtigung der Tatsache, dass alle physikalischen Parameter in den Ausdrücken $B_{1}$ und $B_{2, i}$ positiv sind, siehe Gleichungen (3.20) und (3.21), sind alle fünf Pivotelemente negativ und ungleich Null.

Die drei Pivotelemente $\sigma_{6}$ bis $\sigma_{8}$ haben eine ähnliche Struktur und hier wird beispielhaft das Sechste untersucht:

$$
\sigma_{6}=\underbrace{\underbrace{\frac{B_{2, \mathrm{CO}_{2}}^{2}}{B_{2, \mathrm{CO}_{2}}+B_{1} T_{\mathrm{g}}}}_{<B_{2, \mathrm{CO}_{2}}}-B_{2, \mathrm{CO}_{2}}}_{<0}-\underbrace{B_{3}}_{>0} \underbrace{k_{1}}_{>0} \underbrace{(1-\Theta)}_{>0} .
$$

Mit der Argumentation in Gleichung (A.2) ist die Summe der ersten zwei Terme in Gleichung (A.3) negativ und ungleich Null. Der Sauerstofffüllstand $\Theta$ bewegt sich im Intervall von Null bis Eins und zusammen mit dem positiven Ausdruck $B_{3}$ und der positiven Geschwindigkeitskonstante $k_{1}$ ist der dritte Term im Pivotelement $\sigma_{6}$ immer positiv. Damit ist das Pivotelement als ganzes negativ und ungleich Null. 
Die letzten zwei Pivotelemente $\sigma_{9}$ und $\sigma_{10}$ haben eine ähnliche Struktur, sind jedoch deutlich komplexer als die bisher untersuchten Pivotelemente. Wie bei $\sigma_{6}$ können aber die bisher untersuchten Pivotelemente darin identifiziert und so die bisher gemachten Abschätzungen weiter genutzt werden. Beispielhaft wird das neunte Pivotelement untersucht:

$$
\sigma_{9}=\underbrace{\frac{B_{2, \mathrm{CO}_{2}}^{2}}{B_{2, \mathrm{CO}_{2}}+B_{1} T_{\mathrm{g}}}-B_{2, \mathrm{CO}_{2}}}_{<0}-\underbrace{\Theta B_{3} \frac{k_{1}}{K_{1}}}_{>0}+\frac{\Theta\left(B_{3} k_{1}\right)^{2}(1-\Theta)}{K_{1}\left|\sigma_{6}\right|} .
$$

$K_{1}$ bezeichnet die positive Gleichgewichtskonstante der Reaktion (R1), d. h. an dieser Stelle werden die Gleichgewichtsreaktionen nicht in jeweils zwei Hinreaktionen aufgeteilt. Der Betrag des sechsten Pivotelements wird wie folgt abgeschätzt

$$
\left|\sigma_{6}\right|=\left|\frac{B_{2, \mathrm{CO}_{2}}^{2}}{B_{2, \mathrm{CO}_{2}}+B_{1} T_{\mathrm{g}}}-B_{2, \mathrm{CO}_{2}}\right|+B_{3} k_{1}(1-\Theta)>B_{3} k_{1}(1-\Theta)
$$

und damit eine Abschätzung für den letzten Term in Gleichung (A.4) vorgenommen:

$$
\frac{\Theta\left(B_{3} k_{1}\right)^{2}(1-\Theta)}{K_{1}\left|\sigma_{6}\right|}<\frac{\Theta\left(B_{3} k_{1}\right)^{2}(1-\Theta)}{K_{1} B_{3} k_{1}(1-\Theta)}=\Theta B_{3} \frac{k_{1}}{K_{1}} .
$$

Einsetzen der Abschätzung (A.6) in Gleichung (A.4) liefert auch für $\sigma_{9}$ einen negativen und von Null verschiedenen Wert.

Damit sind alle zehn Pivotelemente unabhängig von der Parameterierung des Katalysatormodells von Null verschieden und die Invertierung der Matrix A ist möglich. Aufgrund der Anforderungen an das Reaktionsschema in Kapitel 3 ist davon auszugehen, dass sich die Struktur der Pivotelemente für andere Reaktionschemata nicht allzu stark verändern wird und dass die Invertierung der Matrix A in allen Fällen möglich ist.

\section{A.4 Beispiel für ein Reaktionsschema mit einer Oberflächenspezies}

Das Reaktionsschema von Weilenmann [111] umfasst die folgenden fünf Reaktionsgleichungen

$$
\begin{aligned}
\mathrm{O}_{2}+2 \mathrm{Ce}_{2} \mathrm{O}_{3} & \rightarrow 2 \mathrm{Ce}_{2} \mathrm{O}_{4}, \\
\mathrm{NO}+\mathrm{Ce}_{2} \mathrm{O}_{3} & \rightarrow \mathrm{Ce}_{2} \mathrm{O}_{4}+0,5 \mathrm{~N}_{2}, \\
\mathrm{CO}+\mathrm{Ce}_{2} \mathrm{O}_{4} & \rightarrow \mathrm{CO}_{2}+\mathrm{Ce}_{2} \mathrm{O}_{3}, \\
\mathrm{CH}_{1,85}+2,925 \mathrm{Ce}_{2} \mathrm{O}_{4} & \rightarrow \mathrm{CO}_{2}+0,925 \mathrm{H}_{2} \mathrm{O}+2,925 \mathrm{Ce}_{2} \mathrm{O}_{3}, \\
\mathrm{H}_{2}+\mathrm{Ce}_{2} \mathrm{O}_{4} & \rightarrow \mathrm{H}_{2} \mathrm{O}+\mathrm{Ce}_{2} \mathrm{O}_{3},
\end{aligned}
$$

mit den acht Gasspezies $\mathrm{O}_{2}$, NO, $\mathrm{CO}, \mathrm{CH}_{1,85}, \mathrm{H}_{2}, \mathrm{~N}_{2}, \mathrm{CO}_{2}$ und $\mathrm{H}_{2} \mathrm{O}$, die in derselben Reihenfolge in den Vektoren $\mathbf{c}$ und $\mathbf{c}^{\text {in }}$ stehen, sowie Ceroxid $\left(\mathrm{Ce}_{2} \mathrm{O}_{3}\right.$ und $\left.\mathrm{Ce}_{2} \mathrm{O}_{4}\right)$ als Oberflächenspezies. Es 
gibt fünf Reaktionsgleichungen, sodass nur für die ersten fünf Gasspezies Gewichtsfunktionen definiert werden können. Wird wie in Kapitel 3 mit $\Theta$ weiterhin der Sauerstofffüllstand einer Zelle des Modells bezeichnet, so gilt für seine Ableitung die folgende Differentialgleichung

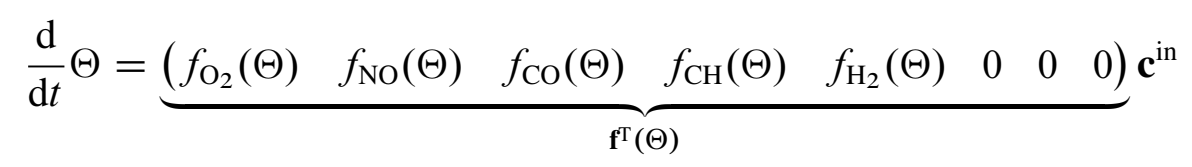

und für die Gaskonzentrationen in der Modellzelle die folgende Matrizengleichung:

$$
\mathbf{c}=\mathbf{G}_{\mathrm{c}} \mathbf{c}^{\mathrm{in}} .
$$

Die Matrix $\mathbf{G}_{\mathrm{c}}$ kann zeilenweise nach Gleichung (3.36) aufgebaut werden und ergibt sich zu

$$
\mathbf{G}_{\mathrm{c}}=k_{0} \mathbf{I}_{8}+k\left(\begin{array}{ccccc}
-\frac{1}{2} f_{\mathrm{O}_{2}} & 0 & 0 & 0 & 0 \\
0 & -f_{\mathrm{NO}} & 0 & 0 & 0 \\
0 & 0 & f_{\mathrm{CO}} & 0 & 0 \\
0 & 0 & 0 & \frac{1}{2,925} f_{\mathrm{CH}} & 0 \\
0 & 0 & 0 & 0 & f_{\mathrm{H}_{2}} \\
0 & \frac{1}{2} f_{\mathrm{NO}} & 0 & 0 & 0 \\
0 & 0 & -f_{\mathrm{CO}} & -\frac{1}{2,925} f_{\mathrm{CH}} & 0 \\
0 & 0 & 0 & -\frac{0,925}{2,925} f_{\mathrm{CH}} & -f_{\mathrm{H}_{2}}
\end{array}\right)
$$

mit der $8 \times 8$-dimensionalen Einheitsmatrix $\mathbf{I}_{8}$ und der $8 \times 3$-dimensionalen Nullmatrix $\mathbf{0}_{8 \times 3}$.

\section{A.5 Beispiel für ein Reaktionsschema mit mehreren Oberflächenspezies}

Das Reaktionsschema von Scheuer u. a. [88] umfasst die folgenden zehn Reaktionsgleichungen

$$
\begin{aligned}
\mathrm{NH}_{3}+\mathrm{b} & \rightarrow \mathrm{NH}_{3}-\mathrm{b}, \\
\mathrm{NH}_{3}-\mathrm{b} & \rightarrow \mathrm{NH}_{3}+\mathrm{b}, \\
\mathrm{O}_{2}+2 \mathrm{a} & \rightarrow 2 \mathrm{O}-\mathrm{a}, \\
2 \mathrm{O}-\mathrm{a} & \rightarrow \mathrm{O}_{2}+2 \mathrm{a}, \\
\mathrm{NH}_{3}-\mathrm{b}+1,5 \mathrm{O}-\mathrm{a} & \rightarrow \mathrm{N}-\mathrm{a}+1,5 \mathrm{H}_{2} \mathrm{O}+0,5 \mathrm{a}+\mathrm{b}, \\
\mathrm{NO}-\mathrm{a} & \rightarrow \mathrm{NO}+\mathrm{a}, \\
\mathrm{NO}+\mathrm{a} & \rightarrow \mathrm{NO}-\mathrm{a}, \\
\mathrm{N}-\mathrm{N}-\mathrm{a}+\mathrm{O}-\mathrm{a} & \rightarrow \mathrm{N}_{2}+2 \mathrm{a}-\mathrm{a}+\mathrm{a}, \\
\mathrm{NO}-\mathrm{a}+\mathrm{N}-\mathrm{a} & \rightarrow \mathrm{N}_{2} \mathrm{O}+2 \mathrm{a},
\end{aligned}
$$

mit den sechs Gasspezies $\mathrm{NH}_{3}, \mathrm{O}_{2}, \mathrm{NO}, \mathrm{H}_{2} \mathrm{O}, \mathrm{N}_{2}$ und $\mathrm{N}_{2} \mathrm{O}$, die in derselben Reihenfolge in den Vektoren $\mathbf{c}$ und $\mathbf{c}^{\text {in }}$ stehen, sowie den sechs Oberflächenspezies a, O-a, N-a, NO-a, b und 
$\mathrm{NH}_{3}$-b. Die Belegungsgrade von a und b können aus den Belegungsgraden der restlichen vier Oberflächenspezies algebraisch ermittelt werden:

$$
\begin{aligned}
& \Theta_{\mathrm{b}}=1-\Theta_{\mathrm{NH}_{3}-\mathrm{b}}, \\
& \Theta_{\mathrm{a}}=1-\Theta_{\mathrm{O}-\mathrm{a}}-\Theta_{\mathrm{N}-\mathrm{a}}-\Theta_{\mathrm{NO}-\mathrm{a}} .
\end{aligned}
$$

Daher enthält jede Zelle des Modells nur vier Zustandsgrößen. Die Differentialgleichung zur Beschreibung einer Modellzelle lautet

$$
\frac{\mathrm{d}}{\mathrm{d} t} \underbrace{\left(\begin{array}{c}
\Theta_{\mathrm{NH}_{3}-\mathrm{b}} \\
\Theta_{\mathrm{O}-\mathrm{a}} \\
\Theta_{\mathrm{N}-\mathrm{a}} \\
\Theta_{\mathrm{NO}-\mathrm{a}}
\end{array}\right)}_{\boldsymbol{\Theta}_{1}}=\mathbf{f}_{0}\left(\boldsymbol{\Theta}_{1}\right)+\left(\begin{array}{cccc}
f_{\mathrm{NH}_{3}}^{\mathrm{NH}_{3}-\mathrm{b}} & 0 & 0 & 0 \\
0 & f_{\mathrm{O}_{2}}^{\mathrm{O}-\mathrm{a}} & 0 & 0 \\
0 & 0 & 0 & 0 \\
0 & 0 & 0 & f_{\mathrm{NO}}^{\mathrm{NO}-\mathrm{a}}
\end{array}\right) \mathbf{0}_{4 \times 2}) \mathbf{c}^{\mathrm{in}}
$$

und aufgrund der vielen Reaktionen zwischen den Oberflächenspezies können nur drei Gewichtsfunktionen aus den Reaktionen (R10), (R12) und (R16) definiert werden. Ein Großteil der Systemdynamik ist in der Vektorfunktion $\mathbf{f}_{0}$ enthalten. Der von den Eingangskonzentrationen abhängige Teil der Gaskonzentrationen in der Zelle kann durch die Gewichtsfunktionen ausgedrückt werden und es resultiert die folgende Gleichung

$$
\mathbf{c}=\mathbf{G}_{\mathrm{c}, 0}\left(\boldsymbol{\Theta}_{1}\right)+\underbrace{\left(k_{0} \mathbf{I}_{6}+k\left(\begin{array}{cccc|c}
-f_{\mathrm{NH}_{3}}^{\mathrm{NH}_{3}-\mathrm{b}} & 0 & 0 & 0 \\
0 & -\frac{1}{2} f_{\mathrm{O}_{2}}^{\mathrm{O}-\mathrm{a}} & 0 & 0 & \\
0 & 0 & 0 & 0 & \mathbf{0}_{4 \times 2} \\
0 & 0 & 0 & -f_{\mathrm{NO}}^{\mathrm{NO}-\mathrm{a}} & \\
\hline 0 & \mathbf{0}_{2 \times 4}
\end{array}\right)\right.}_{\mathbf{G}_{\mathrm{c}}} \mathbf{c}^{\text {in }}
$$

mit dem 6×1-dimensionalen Vektor $\mathbf{G}_{\mathrm{c}, 0}$. 


\section{Literaturverzeichnis}

[1] Acres, G. J. K. und Cooper, B. J.: Automobile Emission Control Systems: Platinum Catalysts For Exhaust Purification. In: Johnson Matthey Technology Review 16.2 (1972), S. 42-47.

[2] Adamy, J.: Nichtlineare Regelungen. Berlin: Springer, 2009.

[3] Allgöwer, F. und Gilles, E. D.: Nichtlinearer Reglerentwurf auf der Grundlage exakter Linearisierungstechniken. In: Nichtlineare Regelung : Methoden, Werkzeuge, Anwendungen. Bd. 1026. VDI-Berichte. Düsseldorf: VDI-Verlag, 1993, S. 209-234.

[4] Auckenthaler, T. S.: Modelling and Control of Three-Way Catalysts. Doktorarbeit. ETH Zürich, 2005.

[5] Balenovic, M.: Modeling and model-based control of a three-way catalytic converter. Doktorarbeit. Technische Universiteit Eindhoven, 2002.

[6] Balenovic, M. und Backx, T.: Model-based predictive control of a three-way catalytic converter. In: Proceedings of the 2001 IEEE International Conference on Control Applications. 2001, S. 626-631.

[7] Balenovic, M., Edwards, J. und Backx, T.: Vehicle application of model-based catalyst control. In: Control Engineering Practice 14.3 (2006), S. 223-233.

[8] Baunach, T., Schänzlin, K. und Diehl, L.: Sauberes Abgas durch Keramiksensoren: Vor 30 Jahren hielt die Lambdasonde Einzug in das Automobil - jetzt auch in Dieselfahrzeuge. In: Physik Journal 5.5 (2006), S. 33-38.

[9] Bekiaris-Liberis, N., Jankovic, M. und Krstic, M.: PDE-based analysis and control of the oxygen storage level in three-way catalytic converters. In: IEEE 51 st Annual Conference on Decision and Control. 2012, S. 3759-3764.

[10] Benninger, N. F. und Rivoir, J.: Ein neues konsistentes Maß zur Beurteilung der Steuerbarkeit in linearen, zeitinvarianten Systemen. In: at - Automatisierungstechnik 34.12 (1986), S. 473-479.

[11] Betz, B.: Entwicklung einer Versuchsanlage für transiente Experimente. Masterarbeit. TU Darmstadt, 2013.

[12] Bidan, P., Boverie, S. und Chaumerliac, V.: Nonlinear control of a spark-ignition engine. In: IEEE Transactions on Control Systems Technology. Bd. 3. 1995, S. 4-13.

[13] Bundesministerium für Bildung und Forschung, Hrsg.: MatRessource: Materialien für eine ressourceneffiziente Industrie und Gesellschaft. 2014.

[14] Burch, R.: Knowledge and Know-How in Emission Control for Mobile Applications. In: Catalysis Reviews 46.3-4 (2004), S. 271-334. 
[15] Camacho, E. F. und Bordons, C.: Model Predictive Control. London, New York: Springer, 2007.

[16] Chatterjee, D.: Detaillierte Modellierung von Abgaskatalysatoren. Doktorarbeit. Ruprecht-Karls-Universität Heidelberg, 2001.

[17] Chatterjee, D., Deutschmann, O. und Warnatz, J.: Detailed surface reaction mechanism in a three-way catalyst. In: Faraday Discussions 119.1 (2001), S. 371-384.

[18] Crucq, A. und Frennet, A.: Catalysis and Automotive Pollution Control. Bd. 30. Amsterdam, New York: Elsevier Science, 1987.

[19] Czeslik, C., Seemann, H. und Winter, R.: Basiswissen Physikalische Chemie. Wiesbaden: Vieweg+Teubner Verlag, 2010.

[20] Datta, A. und Xing, L.: The theory and design of adaptive internal model control schemes. In: Proceedings of the 1998 American Control Conference. Bd. 6, S. 36773684.

[21] Der Rat der Europäischen Gemeinschaften: Richtlinie 70/220/EWG des Rates vom 20. März 1970 zur Angleichung der Rechtsvorschriften der Mitgliedstaaten über Maßnahmen gegen die Verunreinigung der Luft durch Abgase von Kraftfahrzeugmotoren mit Fremdzündüng. 1970.

[22] Der Rat der Europäischen Gemeinschaften: Richtlinie 91/441/EWG des Rates vom 26. Juni 1991 zur Änderung der Richtlinie 70/220/EWG zur Angleichung der Rechtsvorschriften der Mitgliedstaaten über Maßnahmen gegen die Verunreinigung der Luft durch Emissionen von Kraftfahrzeugen. 1991.

[23] Domike, J. R. und Zacaroli, A. C.: The Clean Air Act Handbook. Chicago: American Bar Association, 2011.

[24] Ebbesen, S., Kiwitz, P. und Guzzella, L.: A Generic Particle Swarm Optimization Matlab Function. In: American Control Conference. 2012, S. 1519-1524.

[25] Eichlseder, H., Klüting, M. und Piock, W. F.: Grundlagen und Technologien des Ottomotors. Wien, New York: Springer, 2008.

[26] EU Ad-Hoc Working Group on Raw Materials: Report On Critical RAW Materials For The EU. 2014.

[27] Europäisches Parlament: Richtlinie 1999/94/EG des Europäischen Parlaments und des Rates vom 13. Dezember 1999 über die Bereitstellung von Verbraucherinformationen über den Kraftstoffverbrauch und $\mathrm{CO}_{2}$-Emissionen beim Marketing für neue Personenkraftwagen. 1999.

[28] Europäisches Parlament: Verordnung (EG) Nr. 443/2009 des Europäischen Parlaments und des Rates vom 23. April 2009 zur Festsetzung von Emissionsnormen für neue Personenkraftwagen im Rahmen des Gesamtkonzepts der Gemeinschaft zur Verringerung der $\mathrm{CO}_{2}$-Emissionen von Personenkraftwagen und leichten Nutzfahrzeugen. 2009.

[29] European Commission: Tackling the Challenges in Commodity Markets and on RAW Materials. 2011. 
[30] Fiengo, G., Cook, J. A. und Grizzle, J. W.: Fore-aft oxygen storage control. In: Proceedings of the 2002 American Control Conference. Bd. 2. 2002, S. 1401-1406.

[31] Fiengo, G., Grizzle, J. W. und Cook, J. A.: Experimental Results on Dual-UEGO Active Catalyst Control. In: IFAC Symposium on Advances in Automotive Control. Bd. 2. 2004, S. 343-348.

[32] Föllinger, O. und Konigorski, U.: Regelungstechnik: Einführung in die Methoden und ihre Anwendung. Berlin: VDE-Verlag, 2013.

[33] Franceschi, E. M., Muske, K. R., Peyton Jones, J. C. und Makki, I.: An Adaptive DelayCompensated PID Air Fuel Ratio Controller. In: SAE Technical Paper 2007-01-1342. 2007.

[34] Graichen, K.: Methoden der Optimierung und optimalen Steuerung. Vorlesungsskript. Universität Ulm, 2012.

[35] Gröll, L.: Methodik zur Integration von Vorwissen in die Modellbildung. Karlsruhe: KIT Scientific Publishing, 2015.

[36] Gruden, D.: Umweltschutz in der Automobilindustrie: Motor, Kraftstoffe, Recycling. Wiesbaden: Vieweg + Teubner, 2008.

[37] Guderian, R.: Air Pollution by Photochemical Oxidants: Formation, Transport, Control, and Effects on Plants. Bd. 52. Berlin, Heidelberg: Springer, 1985.

[38] Guo, G., Shane, M., Laing, P. und Jensen, T. E.: Development of a New Oxygen Storage Model for SIMTWC. In: SAE Technical Paper 2007-01-1081. 2007.

[39] Guzzella, L., Simons, M. und Geering, H. P.: Feedback linearizing air/fuel-ratio controller. In: Control Engineering Practice 5.8 (1997), S. 1101-1105.

[40] Guzzella, L. und Onder, C. H.: Introduction to Modeling and Control of Internal Combustion Engine Systems. Berlin: Springer, 2010.

[41] Heywood, J. B.: Internal Combustion Engine Fundamentals. New York: McGraw-Hill, Inc., 1988.

[42] Hofmann, P.: Hybridfahrzeuge. Wien: Springer, 2014.

[43] Inagaki, H., Ohata, A. und Inoue, T.: An adaptive fuel injection control with internal model in automotive engines. In: 16th Annual Conference of IEEE Industrial Electronics Society. 1990, S. 78-83.

[44] Ioannou, P. A. und Sun, J.: Robust Adaptive Control. Upper Saddle River: PTR PrenticeHall, 1996.

[45] Isidori, A.: Nonlinear Control Systems. Berlin, New York: Springer, 1995.

[46] Johnson Matthey, Hrsg.: Platinum 2013: Interim Review. Royston, 2013.

[47] Jones, V. K., Ault, B. A., Franklin, G. F. und Powell, J. D.: Identification and air-fuel ratio control of a spark ignition engine. In: IEEE Transactions on Control Systems Technology. Bd. 3. 1995, S. 14-21. 
[48] Kako, J., Okazaki, S. und Kato, N.: Development of a New Model Based Air-Fuel Ratio Control System. In: ICCAS-SICE. 2009, S. 4134-4139.

[49] Kiencke, U. und Nielsen, L.: Automotive Control Systems: For Engine, Driveline, and Vehicle. Berlin, New York: Springer, 2005.

[50] Kiwic, P.: Model-based control of catalytic converters. Doktorarbeit. ETH Zürich.

[51] Kiwitz, P., Onder, C. und Guzzella, L.: Control-oriented modeling of a three-way catalytic converter with observation of the relative oxygen level profile. In: Journal of Process Control 22.6 (2012), S. 984-994.

[52] Koop, J. und Deutschmann, O.: Detailed surface reaction mechanism for Pt-catalyzed abatement of automotive exhaust gases. In: Applied Catalysis B: Environmental 91.1-2 (2009), S. 47-58.

[53] Koop, J.: Detaillierte Modellierung der PT-katalysierten Schadstoffminderung in Abgasen moderner Verbrennungsmotoren. Doktorarbeit. Karlsruher Institut für Technologie, 2008.

[54] Krener, A. J. und Ide, K.: Measures of Unobservability. In: Proceedings of the 48th IEEE Conference on Decision and Control. 2009, S. 6401-6406.

[55] Litz, L.: Modale Maße für Steuerbarkeit, Beobachtbarkeit, Regelbarkeit und Dominanz: Zusammenhänge, Schwachstellen, neue Wege. In: at - Automatisierungstechnik 31.5 (1983), S. 148-158.

[56] Lofberg, J.: YALMIP: A toolbox for modeling and optimization in MATLAB. In: IEEE International Symposium on Computer Aided Control Systems Design. 2004, S. 284-289.

[57] Love, J.: Process Automation Handbook: A Guide to Theory and Practice. London: Springer, 2007.

[58] Lox, E. S. J. und Engler, B. H.: Environmental Catalysis: Environmental Catalysis Mobile Sources. In: Handbook of Heterogeneous Catalysis. Hrsg. von G. Ertl, H. Knzinger und J. Weitkamp. Weinheim: Wiley-VCH Verlag, 1997, S. 1559-1633.

[59] Lunze, J.: Regelungstechnik 1. Berlin: Springer, 2007.

[60] Lunze, J.: Regelungstechnik 2. Berlin, Heidelberg, New York: Springer, 2008.

[61] Makki, I. H., Surnilla, G., Kerns, J. M. und Smith, S. B.: Engine control and catalyst monitoring with downstream exhaust gas sensors. US6904751 B2. 2005.

[62] McNeill, J. R.: Something New Under the Sun: An Environmental History of the Twentieth-Century World. New York: Norton, 2001.

[63] Mladenov, N.: Modellierung von Autoabgaskatalysatoren. Doktorarbeit. Karlsruher Institut für Technologie, 2009.

[64] Möller, R. J.: Modeling and control of three-way catalysts. Doktorarbeit. ETH Zürich, 2009. 
[65] Möller, R. J., Votsmeier, M., Onder, C., Guzzella, L. und Gieshoff, J.: Is Oxygen Storage in Three-Way Catalysts an Equilibrium Controlled Process? In: Applied Catalysis B: Environmental 91.1-2 (2009), S. 30-38.

[66] Moraal, P. E.: Adaptive compensation of fuel dynamics in an SI engine using a switching EGO sensor. In: Proceedings of the 34th IEEE Conference on Decision and Control. 1995, S. 661-666.

[67] Morari, M. und Zafiriou, E.: Robust Process Control. Englewood Cliffs, New Jersey: Prentice Hall, Inc., 1989.

[68] Muske, K. R., Peyton Jones, J. C. und Franceschi, E. M.: Adaptive Analytical ModelBased Control for SI Engine Air-Fuel Ratio. In: IEEE Transactions on Control Systems Technology 16.4 (2008), S. 763-768.

[69] Muske, K. R. und Peyton Jones, J. C.: Multi-Objective Model-Based Control for an Automotive Catalyst. In: Journal of Process Control 16.1 (2006), S. 27-35.

[70] Odendall, B. und Risse, C.: Verfahren zum Betreiben eines Verbrennungsmotors bei Lambdaregelung, Verfahren zum Ermitteln der Speicherkapazität eines Sauerstoffspeichers in einem Abgasstrang sowie Kraftfahrzeug. DE102008029346 A1. 2009.

[71] Peyton Jones, J. C. und Jackson, R. A.: Potential and Pitfalls in the Use of dual exhaust gas oxygen sensors for three-way catalyst monitoring and control. In: Proceedings of the Institution of Mechanical Engineers, Part D: Journal of Automobile Engineering 217.6 (2003), S. 475-488.

[72] Peyton Jones, J. C. und Schallock, R.: Persistent Memory Effects and the Mid- and PostBrick Dynamic Behaviour of Three-Way Automotive Catalysts. In: Oil \& Gas Science and Technology - Rev. IFP Energies nouvelles 66.4 (2011), S. 667-679.

[73] Peyton Jones, J. C.: Modeling Combined Catalyst Oxygen Storage and Reversible Deactivation Dynamics for Improved Emissions Prediction. In: SAE Techincal Paper 2003-01-0999. 2003.

[74] Peyton Jones, J. C., Roberts, J. B., Pan, J. und Jackson, R. A.: Modeling the Transient Characteristics of a Three-Way Catalyst. In: SAE Technical Paper 1999-01-0460. 1999.

[75] Pischinger, R., Klell, M. und Sams, T.: Thermodynamik der Verbrennungskraftmaschine. Wien: Springer, 2010.

[76] Poling, B. E., Prausnitz, J. M. und O'Connell, J. P.: The Properties of Gases and Liquids. New York: McGraw-Hill, 2001.

[77] Postma, M.: Air-Fuel Ratio Control in Spark Ignition Internal Combustion Engines Using Switching LPV Techniques. Doktorarbeit. The University Of British Columbia, 2011.

[78] Postma, M. und Nagamune, R.: Air-Fuel Ratio Control of Spark Ignition Engines Using a Switching LPV Controller. In: IEEE Transactions on Control Systems Technology 20.5 (2012), S. 1175-1187. 
[79] Ramanathan, K. und Sharma, C. S.: Kinetic Parameters Estimation for Three Way Catalyst Modeling. In: Industrial \& Engineering Chemistry Research 50.17 (2011), S. 9960-9979.

[80] Rao, S. K., Imam, R., Ramanathan, K. und Pushpavanam, S.: Sensitivity Analysis and Kinetic Parameter Estimation in a Three Way Catalytic Converter. In: Industrial \& Engineering Chemistry Research 48.8 (2009), S. 3779-3790.

[81] Reif, K.: Gasoline Engine Management: Systems and Components. Wiesbaden: Springer Vieweg, 2015.

[82] Riege, B.: Strukturmaße für dynamische Systeme. 1998.

[83] Riegel, J., Neumann, H. und Wiedenmann, H.-M.: Exhaust gas sensors for automotive emission control. In: Solid State Ionics 152-153 (2002), S. 783-800.

[84] Roduner, C. A.: $\mathrm{H}_{\infty}$-Regelung linearer Systeme mit Totzeiten. Doktorarbeit. ETH Zürich, 1997.

[85] Roduner, C. A., Onder, C. H. und Geering, H. P.: Automated Design of an Air/Fuel Controller for an SI Engine Considering the Three-Way Catalytic Converter in the $\mathrm{H}$ Infinty Approach. In: Proceedings of the 5th Mediterranean Conference on Control and Systems. 1997, S. 1-7.

[86] Rupp, D. und Guzzella, L.: Adaptive internal model control with application to fueling control. In: Control Engineering Practice 18.8 (2010), S. 873-881.

[87] Schallock, R. W., Muske, K. R. und Peyton Jones, J. C.: Model Predictive Functional Control for an Automotive Three-way Catalyst. In: SAE International Journal of Fuels and Lubricants 2.1 (2009), S. 242-249.

[88] Scheuer, A., Votsmeier, M., Schuler, A., Gieshoff, J., Drochner, A. und Vogel, H.: $\mathrm{NH}_{3}$ Slip Catalysts: Experiments Versus Mechanistic Modelling. In: Topics in Catalysis 52.13-20 (2009), S. 1847-1851.

[89] Schödel, S., Votsmeier, M. und Fischerauer, G.: Microwave-assisted oxygen storage level estimation for three-way catalyst control: Model-based development and benchmarking of selected control strategies. In: The Canadian Journal of Chemical Engineering 92.9 (2014), S. 1597-1606.

[90] Shafai, E., Roduner, C. und Geering, H. P.: Indirect Adaptive Control of a Three-Way Catalyst. In: SAE Technical Paper 961038. 1996.

[91] Shaw, S. und Chegwidden, J.: Global drivers for rare earth demand. Hrsg. von Roskill Information Services Ltd. 2012.

[92] Shelef, M.: Nitric Oxide: Surface Reactions and Removal from Auto Exhaust. In: Catalysis Reviews 11.1 (1975), S. 1-40.

[93] Shelef, M. und McCabe, R.: Twenty-five years after introduction of automotive catalysts: What next? In: Catalysis Today 62.1 (2000), S. 35-50. 
[94] Shelef, M., Jones, J. H., Kummer, J. T., Otto, K. und Weaver, E. E.: Selective catalytic reaction of hydrogen with nitric oxide in the presence of oxygen. In: Environmental Science \& Technology 5.9 (1971), S. 790-798.

[95] Siebertz, K., van Bebber, D. T. und Hochkirchen, T.: Statistische Versuchsplanung: Design of Experiments (DOE). Berlin, Heidelberg: Springer, 2010.

[96] Silva, G. J. und Datta, A.: Adaptive internal model control: The discrete-time case. In: International Journal of Adaptive Control and Signal Processing. Bd. 15. 1999, S. 547555.

[97] Skogestad, S. und Postlethwaite, I.: Multivariable Feedback Control: Analysis and Design. John Wiley \& Sons, 2005.

[98] Soumelidis, M. I. und Stobart, R. K.: Dynamic Modelling of Three-Way Catalysts Using Non-Linear Identification Techniques. In: Proceedings of the Institution of Mechanical Engineers, Part I: Journal of Systems and Control Engineering 220.7 (2006), S. 595-605.

[99] Soumelidis, M. I., Stobart, R. K. und Jackson, R. A.: A Chemically Informed, ControlOriented Model of a Three-Way Catalytic Converter. In: Proceedings of the Institution of Mechanical Engineers, Part D: Journal of Automobile Engineering 221.9 (2007), S. 1169-1182.

[100] Strohrmann, M. und Brunner, U.: Systemtheorie Teil A: Zeitkontinuierliche Signale und Systeme. Vorlesungsskript. Hochschule Karlsruhe Technik und Wirtschaft, 2016.

[101] Thorsten Schulz-Walden: Anfange globaler Umweltpolitik. München: Oldenbourg Wissenschaftsverlag, 2013.

[102] Tomforde, M., Drewelow, W. und Schultalbers, M.: Air-fuel Ratio Control with Respect to Oxygen Storage Dynamics. In: 16th International Conference on Methods and Models in Automation and Robotics. 2011, S. 242-247.

[103] Trovarelli, A.: Catalytic Properties of Ceria and $\mathrm{CeO}_{2}-$ Containing Materials. In: Catalysis Reviews 38.4 (1996), S. 439-520.

[104] Turin, R. C. und Geering, H. P.: Model-Reference Adaptive A/F-Ratio Control in an SI Engine based on Kalman-Filtering Techniques. In: Proceedings of the 1995 American Control Conference. 1995, S. 4082-4090.

[105] Twigg, M. V.: Progress and future challenges in controlling automotive exhaust gas emissions. In: Applied Catalysis B: Environmental 70.1-4 (2007), S. 2-15.

[106] van Basshuysen, R.: Handbuch Verbrennungsmotor: Grundlagen, Komponenten, Systeme, Perspektiven. Wiesbaden: Springer Vieweg, 2015.

[107] van Basshuysen, R.: Ottomotor mit Direkteinspritzung: Verfahren, Systeme, Entwicklung, Potenzial. Wiesbaden: Springer Vieweg, 2013.

[108] Voigt, C. und Adamy, J.: Formelsammlung der Matrizenrechnung. München: Oldenbourg Verlag, 2007. 
[109] Wächter, A. und Biegler, L. T.: On the implementation of an interior-point filter linesearch algorithm for large-scale nonlinear programming. In: Mathematical Programming 106.1 (2006), S. 25-57.

[110] Warnatz, J. und Maas, U.: Technische Verbrennung. Berlin, Heidelberg: Springer, 1993.

[111] Weilenmann, M.: Aspects of highly transient catalyst simulation. In: Catalysis Today 188.1 (2012), S. 121-134.

[112] Yildiz, Y., Annaswamy, A., Yanakiev, D. und Kolmanovsky, I.: Adaptive air fuel ratio control for internal combustion engines. In: American Control Conference. 2008, S. 2058-2063. 


\section{Betreute studentische Arbeiten}

[113] Baron, R., Yamaoka, L. F., Marangoni, R. R. und Valle, A. M.: Instandsetzung, Modellbildung und Regelung eines inversen Pendels. Projektseminar. TU Darmstadt, 2012.

[114] Dück, D.: Implementierung und Untersuchung von Verfahren zur Lambda- und Sauerstofffüllstandsregelung von Drei-Wege-Autoabgaskatalysatoren. Bachelorarbeit. TU Darmstadt, 2013.

[115] Erben, A.: Analyse und Entwurf einer Regelung zur Ansteuerung einer Lenkmaschine für fahrdynamische Manöver. Diplomarbeit. TU Darmstadt, 2012.

[116] Fuchs, J. P., Schwarz, J. und Cao, G.: Modelling, parameter identification and optimization of the control structure of a mass flow controller. Projektseminar. TU Darmstadt, 2013.

[117] Gora, D.: Linearisierung der Systemgleichungen und Untersuchung der Beobachtbarkeit eines Drei-Wege-Katalysators. Diplomarbeit. TU Darmstadt, 2014.

[118] Huschka, D.: Aufstellen und Lösen des Optimalsteuerungsproblems für einen DreiWege-Autoabgaskatalysator. Studienarbeit. TU Darmstadt, 2015.

[119] Irwanto, S.: Hysteresekompensation und Reglerentwurf für ein Proportionalventil. Diplomarbeit. TU Darmstadt, 2014.

[120] Kemmerzell, M.: Temperaturregelung eines Durchflussreaktors. Bachelorarbeit. TU Darmstadt, 2014.

[121] Liu, Y.: Untersuchung von Verfahren zur robusten Lambdaregelung von Ottomotoren. Masterarbeit. TU Darmstadt, 2014.

[122] Luff, M.: Entwurf einer Vorsteuerung für den Lambdaregelkreis eines Ottomotors. Bachelorarbeit. TU Darmstadt, 2015.

[123] Protasiewicz, W.: Entwurf eines linear-quadratischen Reglers für einen Drei-WegeAutoabgaskatalysators. Studienarbeit. TU Darmstadt, 2015.

[124] Winter, H.: Untersuchung und Implementierung von Verfahren zur Parameteridentifikation eines Drei-Wege-Autoabgaskatalysators. Masterarbeit. TU Darmstadt, 2014. 


\section{Eigene Veröffentlichungen}

[125] Bauer, M., Stanchev, R. und Isermann, R.: Bestimmung der Übergrundgeschwindigkeit aus Fahrdynamiksensoren und Satelliten-Navigationsdaten. In: Autoreg. Bd. 2135. VDIBerichte. Düsseldorf: VDI-Verlag, 2011, S. 401-412.

[126] Konigorski, U., Stanchev, R. und Sieber, U.: Haptisches Kraftfahrzeug-Fahrpedal mit elastisch angekoppeltem Aktuator sowie Verfahren und Regelungseinheit zum Regeln desselben. WO 2015/010816 A2. 2015.

[127] Stanchev, R. und Konigorski, U.: Abgasnachbehandlung bei Ottomotoren: Neuartige Modellbildung und darauf basierende Regelung eines Drei-Wege-Autoabgaskatalysators. In: Autoreg. Bd. 2233. VDI-Berichte. Düsseldorf: VDI-Verlag, 2015, S. 329-340.

[128] Stanchev, R., Konigorski, U. und Votsmeier, M.: Modellbildung und Vergleich von linearen und nichtlinearen Regelungskonzepten für Drei-Wege-Abgaskatalysatoren. In: at - Automatisierungstechnik 64.4 (2016), S. 297-311.

[129] Stanchev, R., Rink, J., Konigorski, U., Vogel, H. und Votsmeier, M.: Systematic Transformation of Chemical Catalyst Models for Control Design. In: IEEE Transactions on Control Systems Technology zur Veröffentlichung angenommen (2016). 


\section{Eigene Vorträge}

[130] Stanchev, R.: Modellbildung und Regelung von Drei-Wege-Autoabgaskatalysatoren. Dresdner Automatisierungstechnische Kolloquien. Dresden, 2016.

[131] Stanchev, R. und Konigorski, U.: Abgasnachbehandlung bei Ottomotoren: Neuartige Modellbildung und darauf basierende Regelung eines Drei-Wege-Autoabgaskatalysators. Robert Bosch GmbH. Schwieberdingen, 2015.

[132] Stanchev, R. und Konigorski, U.: Abgasnachbehandlung bei Ottomotoren: Vergleich linearer und nichtlinearer Regelungskonzepte für einen Drei-WegeAutoabgaskatalysator. Karlsruher Regelkreis. Erlangen, 2015.

[133] Stanchev, R. und Konigorski, U.: Modellbildung, Systemanalyse und Regelung eines Drei-Wege-Autoabgaskatalysators. GMA-Fachausschuss 1.40 - Theoretische Verfahren in der Regelungstechnik. Anif, Österreich, 2014.

[134] Stanchev, R. und Konigorski, U.: Modellbildung und Vergleich von linearen und nichtlinearen Regelungskonzepten für Drei-Wege-Autoabgaskatalysatoren. 50. Regelungstechnisches Kolloquium. Boppard, 2016.

[135] Stanchev, R., Rink, J., Konigorski, U. und Votsmeier, M.: Design of chemical models for model-based control of exhaust gas emissions. MODEGAT: Modeling of Exhaust-Gas After-Treatment 2015. Bad Herrenalb/Karlsruhe, 2015. 


\section{Lebenslauf}

\section{Persönliche Daten}

$\begin{array}{ll}\text { Name } & \text { Radoy Stanchev } \\ \text { Geburtsdatum } & \text { 10. Februar 1985 } \\ \text { Geburtsort } & \text { Sevlievo, Bulgarien } \\ \text { Nationalität } & \text { bulgarisch, deutsch }\end{array}$

Berufstätigkeit

2011-2016 Wissenschaftlicher Mitarbeiter

Technische Universität Darmstadt

Institut für Automatisierungstechnik und Mechatronik

FG Regelungstechnik und Mechatronik

Prof. Dr.-Ing. Ulrich Konigorski

Schul- und Berufsbildung

2005-2011

2004-2005

1999-2003
Diplom-Ingenieur Elektrotechnik und Informationstechnik

Technische Universität Darmstadt

Vertiefungsrichtung: Automatisierungstechnik

Feststellungsprüfung $\mathbf{T}$

Studienkolleg für ausländische Studierende der Technischen Universität

Darmstadt

\section{Diplom für Oberschulabschluss}

Fachoberschule „General Ivan Bachvarov“

Sevlievo, Bulgarien

Vertiefungsrichtung: Elektrotechnik 